\title{
On the origin of species in Termitomyces
}

\section{Leonard J.J. van de Peppel}

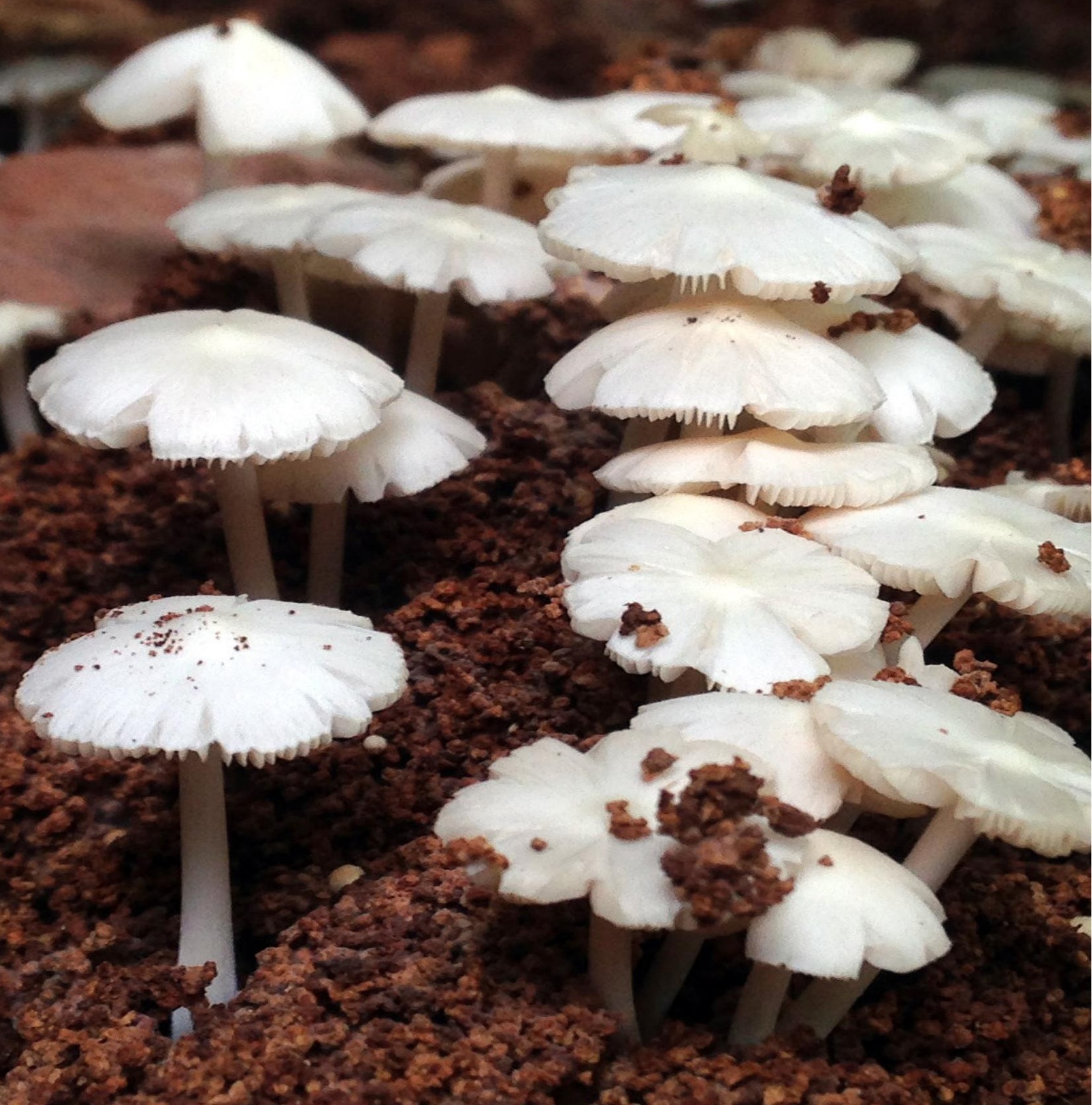




\section{Propositions}

1. The ancestor of Termitomyces was predisposed to become domesticated.

(this thesis)

2. Taxonomy of the genus Termitomyces is greatly hampered by the current taxonomical code.

(this thesis)

3. The peer-review process should be made open.

4. To prevent the general public from misunderstanding evolution as a 'great chain of being', it is necessary to abandon words such as 'basal' or 'advanced' to describe extant organisms.

5. Children and adolescents should learn at school how to critically assess sources to avoid acceptance of fake news or pseudoscientific beliefs.

6. Practicing endurance sports is the physical equivalent of a PhD trajectory.

7. A 30-hour work week should become the norm rather than the exception.

Propositions belonging to the thesis, entitled

On the origin of species in Termitomyces

Leonard J.J. van de Peppel

Wageningen, 19 October 2021 


\section{On the origin of species in Termitomyces}

Leonard J.J. van de Peppel 


\section{Thesis committee}

\section{Promotors}

Prof. Dr B.J. Zwaan

Professor of Genetics

Wageningen University \& Research

Prof. Dr D.K. Aanen

Personal chair at the Laboratory of Genetics

Wageningen University \& Research

\section{Other members}

Prof. Dr T.J.G. Ettema, Wageningen University \& Research

Dr J. Nuytinck, Naturalis Biodiversity Center, Leiden

Prof. Dr P.W. Crous, KNAW - Westerdijk Fungal Biodiversity Institute, Utrecht

Prof. Dr F.P.M. Govers, Wageningen University \& Research

This research was conducted under the auspices of the C.T. de Wit Graduate School for Production Ecology and Resource Conservation. 


\title{
On the origin of species in Termitomyces
}

\author{
Leonard Johannes Jan van de Peppel
}

\section{Thesis}

submitted in fulfilment of the requirements for the degree of doctor at Wageningen University

by the authority of the Rector Magnificus,

$$
\text { Prof. Dr A.P.J. Mol, }
$$

Thesis Committee appointed by the Academic Board

to be defended in public

on Tuesday 19 October 2021

at 4 p.m. in the Aula 
Leonard Johannes Jan van de Peppel

On the origin of species in Termitomyces

182 pages

PhD thesis, Wageningen University, Wageningen, the Netherlands (2021) With references, with summary in English

ISBN: 978-94-6395-919-3

DOI: $10.18174 / 550803$ 


\section{Table of contents}

$\begin{array}{lll}\text { Chapter } 1 & \text { General introduction } & 7\end{array}$

Chapter 2 Ancestral predisposition toward a domesticated lifestyle in the termite-cultivated fungus Termitomyces

Chapter 3 Genetic population structure of the agaric

Blastosporella zonata reveals cryptic species and different roles for sexual and asexual spores in dispersal

Chapter 4 Four new genera and six new species of 59

Lyophylloid agarics (Agaricales, Basidiomycota) from three different continents

Chapter 5 Species and speciation in the termite-cultivated 79 fungus Termitomyces

Chapter 6 High diversity and low host-specificity of Termitomyces symbionts cultivated by Microtermes spp. indicate frequent symbiont exchange

Chapter 7 Termitomyces cryptogamus sp. nov. associated with Macrotermes natalensis in Africa

Chapter 8 General discussion

References

Summary

Acknowledgements 



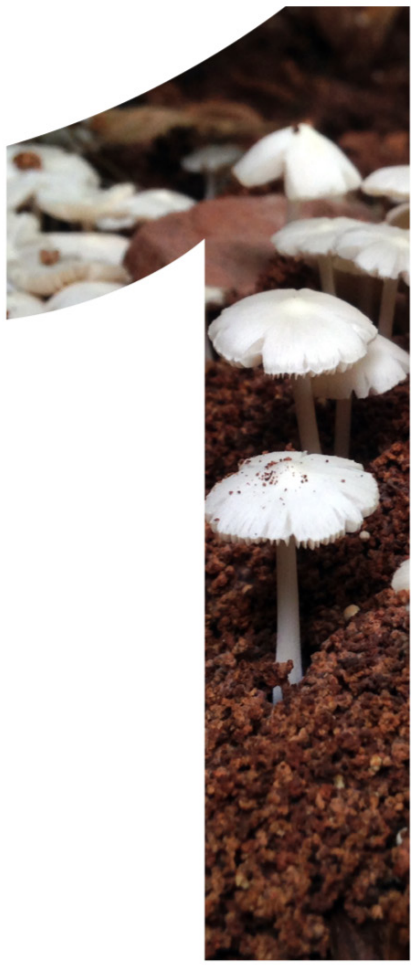

General introduction 


\section{Symbiosis}

The word symbiosis is derived from the Greek words 'syn' and 'bios' meaning "living together". In biology it is defined as a close, long-term interaction between two different organisms. Such an interaction can be commensal when one party benefits with a neutral effect on the other party (so no benefit or harm), parasitic, or mutualistic. An example of a commensal interaction are remora fish which attach themselves to sharks and eat food leftovers while the shark does not gain any benefits nor negative effects. Parasitism is usually associated with bacterial, fungal, or viral diseases and ectoparasites live and feed on the host. Examples of this are fleas, lice, ticks or, in the case of plants, root parasites such as broomrapes (Orobanchaceae). In a mutualistic symbiosis both parties receive a benefit. An important example of mutualistic symbiosis is mycorrhiza, a mutualistic symbiosis between plant roots and mycorrhizal fungi. The plant provides the fungus with sugars in exchange for minerals. Another similar example are nitrogen fixing Rhizobium bacteria in the root nodules of legumes, which fix nitrogen from the air in exchange for sugars.

The main topic of my thesis is insect fungiculture; a mutualistic symbiosis where insects provide a protective growth environment for fungi, in exchange for nutritional benefits. Mutualistic symbioses can be directly linked to major evolutionary innovations (Szathmáry and Maynard-Smith 1995). Lynn Margulis proposed the idea that symbiosis in some cases, over a long time-span could lead to symbiogenesis (Sagan 1967). Symbiogenesis, also known as the endosymbiosis theory, is often used to explain how eukaryotes have evolved. Evidence for this are organelles such as the mitochondrion and the chloroplasts in plants which are thought to have evolved from a free-living prokaryotic ancestor.

\section{The evolution and stability of mutualisms}

From an evolutionary perspective mutualistic symbioses are very unlikely to evolve as selection would favour an interaction which benefits the individual without paying the costs over an interaction with reciprocal benefits. Yet, in the natural world we see plenty of mutualistic symbioses, some of which have been mentioned in the previous paragraph. Theoretically, it is expected that mutualists may abandon or start exploiting their partner and thus turn the mutualistic interaction into a parasitic one (Sachs and Simms 2006). Yet, parasitic relationships which have turned into a mutualistic relationship have been reported, a well-known example being bacteria of the genus Wolbachia which infect the cells of a large range of arthropod host species. Estimates 
vary, but potentially more than half of the total insect population could be infected (Hilgenboecker et al. 2008). Wolbachia infections are usually viewed as parasitic as the bacteria can manipulate the reproductive success of the host in a negative way, for example by causing a female-biased sex ratio, which benefits the bacteria since those are maternally transmitted (Stouthamer, Breeuwer, and Hurst 1999). However, in some cases the opposite effect has been demonstrated as curing the infection can lead to reduced fecundity or even loss of reproductive abilities (Pannebakker et al. 2007). This shift from parasitism towards mutualism has been explained by the effects of compensatory evolution (Aanen and Hoekstra 2007). Another positive effect of a Wolbachia infection is increased resistance of the host against viral infections (Hedges et al. 2008).

Several models have been proposed to explain the evolution of mutualistic symbioses, but there is no general theory. Yet, several factors facilitating the evolution mutualistic symbioses have been identified (Axelrod and Hamilton 1981, Frank 1997, Herre et al. 1999, Sachs et al. 2004, Foster and Wenseleers 2006). These factors include partner choice (choosing a more cooperative partner) (Bull and Rice 1991) and sanctioning of noncooperative behaviour (Kiers et al. 2003). Furthermore, transmission mode has been proposed to be another important determinant in mutualistic symbioses (Frank 1996b). Vertical transmission is when a symbiont is passed from parent to offspring. The opposite, horizontal transmission, is when a symbiont is obtained from an unrelated host or from the environment. Uniparental vertical transmission is thought to be one of the main determinants of directing the interaction between different species towards mutualistic symbiosis as it aligns reproduction of host and symbiont, reduces the costs of finding a symbiont, reduces competition in the symbiont population, and creates co-dependency between host and symbiont (Frank 1997, Law and Dieckmann 1998, Herre et al. 1999). When host and symbiont reproduction are aligned a partner-fidelity feedback can occur so that cooperative symbionts are selected (Bull and Rice 1991, Sachs et al. 2004). In the three main groups of fungus-growing insects; fungusgrowing ants, known as attine ants, fungus-growing beetles, collectively known as ambrosia beetles and fungus-growing termites, uniparental vertical transmission has evolved, which reflects its importance in the stabilization of mutualistic symbioses. Vertical transmission is the main mode of transmission in attine ants (Mueller, Rehner, and Schultz 1998) and ambrosia beetles (Francke-Grosmann 1967, van de Peppel, Aanen, and Biedermann 2018) and has evolved independently in two lineages of fungus-growing termites (Johnson 1981, Korb and Aanen 2003, 
Nobre, Fernandes, et al. 2011). Horizontal transmission may not be reciprocally beneficial as a symbiont is able to escape unfavourable conditions created by the host or the host is able to obtain a more suitable new symbiont. Nevertheless, it is possible to evolve stable mutualisms under horizontal symbiont transmission, the fungusgrowing termites are a good example of this.

Most evolutionary research on mutualisms focus on the stability of mutualistic symbioses (Axelrod and Hamilton 1981, Frank 1994, 1997), and the knowledge on stability could help explaining how a symbiosis can further evolve. However, it cannot predict how it originates. My thesis focusses on this origin of symbioses, an area of much less research.

\section{Insect-fungal symbioses}

Symbioses between insects and fungi are probably less common than symbioses between insects and bacteria. Currently a total of 33 independent origins of ectosymbiotic mutualistic interactions between insects and fungi are known, 21 with Ascomycete fungi and 12 with Basidiomycete fungi (Biedermann and Vega 2020).

Different types of insect-fungus mutualisms can be distinguished. As fungi are sessile organisms they often rely on external factors for dispersal of their spores. Many fungi disperse their spores by wind, but in some cases spores can also be dispersed via insects (Malloch and Blackwell 1992). Fruiting bodies of these fungi may be adapted to attract the host insect and the spores may have evolved to stick to the insect host. A good example of this is a fungus commonly found in Europe; Phallus impudicus, the common stinkhorn. The fruiting bodies of this fungus have a particular smell which attracts mainly dipterans but other insects as well. The insects will land on a sticky spore mass at the tip of the fruiting body and pick up the spores (Ingold 1953). Some species of flies may also eat the sticky spore mass and disperse the spores via their faeces (Schremmer 1963). Symbiotic fungi can also play a role in protection of the host. A species of attine ants, Mycetosoritis clorindae, cover hibernating workers in a layer of live fungal mycelium, and the fungus may prevent the workers from drying out and protect them from natural enemies and disease (Mueller, Ortiz, and Bacci 2010). Another example are Lasius ants which use fungi to reinforce their carton nests (Schlick-Steiner et al. 2008).

Probably the most well-studied type of insect-fungus mutualisms involve nutrition. The symbiotic fungus can be consumed directly for energy, proteins or essential nutrients, but can also break down 
compounds which are otherwise indigestible for the host. An example of a nutritional mutualism is between Sirex woodwasps and basidiomycete fungi of the genus Amylostereum (Talbot 1977). Fungal spores are carried by the female wasp in a special gland (mycangium) and are secreted in a hole in the host tree where eggs are being deposited as well (Slippers, De Groot, and Wingfield 2011). The exact role of the symbiotic fungus in the nutrition of the woodwasps is not entirely clear, but most likely the fungus helps in digesting the wood making it more accessible for the larva (Slippers, Hurley, and Wingfield 2015).

In three insect groups an obligate nutritional symbiosis has evolved between an insect lineage and a fungus or multiple lineages of fungi along with a high degree of sociality in the insect host: in ambrosia beetles, attine ants, and termites.

\section{Ambrosia beetles}

Named after the Greek 'food of the gods', Ambrosia beetles farm fungi as their sole source nutrition inside dead or, in rare cases, living wood. Fungiculture in Ambrosia beetles has evolved many times independently, probably more than a dozen times in the family of weevils (Curculionidae: Scolytinae, Platypodinae)(Hulcr and Stelinski 2017, Vanderpool, Bracewell, and McCutcheon 2018) and a single time in the ship-timber beetles (Lymexylidae)(Francke-Grosmann 1967). The subfamily of ambrosia beetles Platypodinae represents the oldest example of insect-fungiculture with an estimated origin of fungiculture about 96 million years ago (Vanderpool, Bracewell, and McCutcheon 2018). In general, ambrosia beetles cultivate ascomycete fungi, but two ambrosia beetle genera are known to cultivate a single basidiomycete fungus (Li et al. 2017). The ambrosia fungi cultivated by the ambrosia beetles are also polyphyletic, the most well-known fungi are found in the Ascomycete genera Ambrosiella, Dryadomyces and Raffaelea (Alamouti, Tsui, and Breuil 2009). The ambrosia beetles represent the only group in which fungiculture has evolved several times and where multiple unrelated fungi have been domesticated.

Ambrosia beetles have evolved vertical transmission of their symbionts, as the founder females carry the fungus in a special pouch called the mycangium or mycetangium (Francke-Grosmann 1956, Batra 1963). Mycangia differ greatly in morphology and size over various beetle lineages and in some cases there is a certain degree of coevolution between the mycangium and the fungal symbiont (Mayers et al. 2020). Eusociality has evolved in ambrosia beetles (Kent and Simpson 1992), but is rare. Most ambrosia beetles have a division of 
labour but there are no sterile castes like in other social insects such as bees, ants or termites, a type of sociality called subsociality (Biedermann and Taborsky 2011).

\section{Attine ants}

Fungus-growing ants are a monophyletic group of ants (Formicidae) exclusively found in the neotropics. Fungiculture evolved about 55-65 million years ago in the tropical rainforests of South America (Ješovnik, González, and Schultz 2016, Branstetter et al. 2017). Fungiculture evolved once in this group and there have been at least three fungal domestication events (Biedermann and Vega 2020). Although fungiculture has a single origin in this group, five different types of fungiculture have evolved: 'lower agriculture', coral-fungus agriculture, yeast-agriculture, generalized 'higher agriculture' and leaf-cutter agriculture (Mehdiabadi and Schultz 2010). The majority of the fungal symbionts cultivated by fungus-growing ants belong to the tribe Leucocoprineae (family: Lepiotaceae) except for a group of fungusgrowing ants in the genus Apterostigma which started cultivating fungi in the unrelated coral-mushroom family Pterulaceae (Munkacsi et al. 2004, Mehdiabadi and Schultz 2010). Most fungus-growing ants cultivate their fungus on dead plant material and free-living populations of their symbiotic fungus exist (Vo, Mueller, and Mikheyev 2009). Because of these free-living populations it is hard to estimate the exact number of independent domestication events. Only the symbionts of the leafcutter ants are fully domesticated and thus do not appear as free-living outside the nest (Schultz et al. 2015). The two leafcutter genera, Acromyrmex and Atta, cut pieces of live vegetation on which they cultivate their fungal symbiont. The fungus of the leafcutter ants produces nutrient-rich swollen hyphae called gongylidia which are consumed by the larvae (De Fine Licht, Boomsma, and Tunlid 2014). The gongylidia also produce conidia (asexual spores) which serve as an inoculum. Uniparental vertical transmission is the dominant mode of symbiont transmission in fungus-growing ants, if a new colony is founded the queen brings a small bolus of fungus with her to inoculate the new fungus garden (Weber 1972).

\section{Fungus-growing termites}

The fungus-growing termites (Macrotermitinae) are a subfamily of termites which cultivate basidiomycetes of the genus Termitomyces. The symbiosis originated about 30 million years ago in the rainforests of central Africa (Aanen and Eggleton 2005, Roberts et al. 2016). Fungus-growing termites extended their range to almost the entire 
continent of Africa including Madagascar and also migrated to Asia (Bignell, Roisin, and Lo 2010, Nobre, Eggleton, and Aanen 2010). They are the only example of a symmetrical fungus-farming insect system as fungiculture evolved once and a single successful domestication event of their symbionts is known (Aanen et al. 2002).

The termites grow their fungus inside the nest on honeycomblike structures called the fungus comb. The fungus comb is constructed by stacking together faecal pellets from primary faeces which have quickly passed the termite gut. Initial inoculation of the fungus comb occurs via basidiospores (sexual spores) which are presumably present on the plant material that is collected and ingested by the workers. After successful colonization of the fungus comb, the fungus produces asexual fruiting bodies, called nodules, which are consumed by the termites. The nodules contain conidia which are used for constant reinoculation of newly added layers of the fungus comb.

\section{Symbiont transmission mode in fungus-growing termites}

As mentioned earlier, uniparental vertical transmission is an important stabilizing factor as it can reduce host-symbiont conflicts and is expected to evolve over time in repeated mutualistic interactions (Frank 1997). This is reflected by the biology of the three main insect fungusgrowing systems mentioned above, as uniparental vertical transmission has evolved in all three systems. While it is the dominant mode of transmission in ambrosia beetles and fungus-growing ants, in the fungus-growing termites it is relatively rare as it evolved independently in only two lineages (Johnson 1981, Nobre, Fernandes, et al. 2011). Horizontal transmission via sexual spores is maintained as the dominant mode of transmission in fungus-growing termites. It is currently not known why horizontal transmission has been maintained as the dominant mode of symbiont transmission in fungus-growing termites and how reproductive interests of host and symbiont are aligned. Fungus-growing termites cultivate their fungal symbiont as a monoculture within their nests (Katoh et al. 2002, Moriya et al. 2005, Makonde et al. 2013) which is probably the result of frequencydependent selection and may play an important role in maintaining the stability of the symbiosis (Aanen et al. 2009).

\section{Origins of fungiculture}

Two main models have been suggested for the evolution of fungiculture; the "consumption-first" model and the "transmission-first" model (Mueller et al. 2001, Mueller et al. 2005). The consumption-first model suggests that an insect first consumes the fungus as a part of its 
regular diet and later becomes adapted to cultivate the fungus after which transmission follows. In the transmission-first model the insect is first vectoring the fungus and at some point starts to derive nutritional benefits from it after which it becomes cultivated.

The transmission-first model is used to explain some of the many origins of fungiculture in ambrosia beetles as their relatives are phloemfeeding weevils known to vector fungal spores and some ambrosia beetle symbionts are closely related to free-living fungi which are vectored by insects (Mueller et al. 2005, Harrington 2005). Initially, the fungus may have been vectored by beetles, gaining easy access to the tree. Eventually the beetles started to derive nutrition from the fungus, which led to domestication. However, in other cases the consumptionfirst route seems more likely as nutritional dependencies occur in related beetle species and thus may have predated fungiculture (Klepzig and Six 2004, Mueller et al. 2005). For the attine ants it is not clear which of the two models provides the best explanation for the pathway towards domestication (Mueller et al. 2001). The consumptionfirst model has been suggested as the most likely scenario for the origin of the termite-fungus symbiosis because many wood feeding termites show attraction to rotting wood (Rouland-Lefèvre 2000, Mueller et al. 2005) and the consumption of fungus-infested wood may increase termite survival (Viana et al. 2018). Fungus-growing termites are also known to consume the mushrooms of other fungi than Termitomyces outside of their nest (Chiu et al. 2019).

\section{Speciation in fungus-growing termites and Termitomyces}

After the inception of the symbiosis in the rainforest of central Africa (Aanen and Eggleton 2005, Roberts et al. 2016) the fungus-growing termites and Termitomyces expanded their range outside the ancestral rainforest habitat to colonize almost the entire African continent as well as parts of the Asian continent. Speciation events occurred for both the fungus-growing termites as well as Termitomyces resulting in over around 330 described species of fungus-growing termites (Eggleton et al. 2000) and around 40 species of Termitomyces (Kirk et al. 2008). This is remarkable as the sister group of the Macrotermitinae consists of only a single extant species. This species, Sphaerotermes sphaerothorax, cultivates lignocellulolytic bacteria instead of fungi (Garnier-Sillam et al. 1989, Bucek et al. 2019). This difference in extant diversity between the sister groups suggests that fungiculture may have opened a plethora of different niches and has resulted in adaptive radiation.

A significant part of the diversity in the genus Termitomyces remains undescribed (Osiemo et al. 2010), as fruiting in some species 
is rare or may even be absent (Wood and Thomas 1989, Darlington 1994, Bingham 2002). Current morphology-based taxonomy is biased towards species which regularly produce mushrooms and therefore alternative ways to describe species in Termitomyces, involving laboratory cultures and molecular data, have been suggested (Makonde et al. 2013).

\section{This thesis}

In this thesis I focused on the origin and subsequent evolution of fungiculture in the fungus-growing termites. The fungus-growing termites represent the only symmetrical insect fungicultural system meaning fungiculture evolved once and only a single lineage of fungus has been successfully domesticated with no subsequent reversals to a non-symbiotic lifestyle for either partner (Aanen et al. 2002). In the case of fungus-growing ants or ambrosia beetles the traits required in the ancestor of domesticated fungi may have been more general than the traits present in the pre-domesticate ancestor of Termitomyces, as these traits are probably shared among different fungal families from which the symbionts originate. In contrast, the single successful domestication in the fungus-growing termites suggests a specific trait or a set of traits which may have predisposed the ancestor of Termitomyces towards domestication. To investigate whether such a predisposition may have been present in the ancestor of Termitomyces I reconstructed the biology of the ancestor by studying the biology of related taxa. To reconstruct the events leading towards domestication I also reconstructed the phylogeny of Termitomyces its closest living relatives. The sister group of Termitomyces is currently represented by a single species, Tephrocybe rancida, which is supposed to be a saprotroph (Froslev et al. 2003, Hofstetter et al. 2014). Other studies have found a potentially close relationship of the two neotropical genera Blastosporella and Arthromyces to Termitomyces (Baroni et al. 2007, Bellanger et al. 2015). The exact phylogenetic position as well as the biology of these genera was unknown and was investigated in this thesis. With these recently described taxa the monophyly of the genus Termitomyces also could be confirmed with more certainty, which was crucial to infer the domestication process.

The evolution of fungiculture and the subsequent domestication of Termitomyces has resulted in an increase in diversity in both the fungus-growing termites and their symbiotic fungi. The second aim of this thesis was to estimate the current diversity in Termitomyces by estimating the current number of species using a molecular species concept. In addition, I attempted to identify which factors and events 
led to speciation within the genus Termitomyces making it such a highly diverse genus as it is today.

\section{Thesis outline}

To make any inferences on the biology of the ancestor of Termitomyces it is crucial to determine its closest living relatives. In chapter 2 । sampled species from the neotropical genera Arthromyces and Blastosporella which had been shown to be closely related to Termitomyces (Bellanger et al. 2015). Next to sampling tissue material for DNA extraction and subsequent sequencing purposes $I$ also managed to gain information on the biology of these species. In addition I collected five additional unidentified species of Tephrocybe which showed morphological resemblance to either $T$. rancida, $B$. zonata or the genus Arthromyces. Previous studies on the Lyophyllaceae did not resolve all phylogenetic relationships within this group (Hofstetter et al. 2002, Hofstetter et al. 2014, Bellanger et al. 2015) therefore, I sampled representative species of the major genera within the Lyophyllaceae. To confirm the monophyly of Termitomyces I added a set of 25 species of Termitomyces to this dataset. As previous studies used up to five genetic markers, I opted for a phylogenomic approach using a large set of conserved homologues genes.

Species in genus Arthromyces and B. zonata are reported to produce asexual spores (Baroni et al. 2007). Although they share this feature with Termitomyces, the asexual spores in these species are produced on the fruiting body. In chapter 3 I hypothesize that the conidia from Arthromyces and $B$. zonata function mostly for local dispersal of the fungus. During fieldwork in Colombia I collected 21 mushrooms of $B$. zonata from three different sites. By sequencing two highly variable genetic markers I looked for signatures of clonality within and between sites and differentiation between sites. This showed that asexual spores are mainly used for local dispersal and sexual spores also for long-range dispersal. I also identified the presence of two cryptic species.

During the studies in chapter 2, five unidentified species of Tephrocybe were sampled. Four of these species did not match to any species descriptions in current literature and I found that all five species are nested in the same clade as Termitomyces. In chapter 4 I formally describe these unidentified Tephrocybe species as new to science. In addition, I describe a new species of Arthromyces from Guyana and the species Tricholoma furcatifolium is taxonomically re-evaluated as part of its description matches to the fifth unidentified species of Tephrocybe. 
After the origin of the symbiosis in the rainforests of central Africa (Aanen and Eggleton 2005, Roberts et al. 2016), the fungus-growing termites expanded their range to colonize sub-Saharan Africa, Madagascar and parts of Asia. This range expansion was accompanied by an increase in species in both the fungus-growing termites and their Termitomyces symbionts. In chapter 5 I looked at the genus Termitomyces in more detail, and estimated based on genetic species delimitation on a large set of a highly variable genetic marker that there are currently 87 species. From a phylogenetic analysis on these species I recovered five main symbiont clades. In general, each clade was associated with one clade of fungus-growing termite. At the level of individual species, host-specificity was very low. I found that geographic separation, symbiont transmission mode, host control over symbiont fruiting and fruiting mode may influence speciation in Termitomyces.

Uniparental vertical transmission is the predominant symbiont transmission mode in the other two obligate insect fungiculture systems (attine ants and ambrosia beetles). In fungus-growing termites horizontal transmission is the dominant transmission mode and uniparental vertical transmission has only evolved in the species Macrotermes bellicosus (via the male reproductive) and in the genus Microtermes (via the female reproductive)(Korb and Aanen 2003). Theoretically it is expected that uniparental vertical transmission increases host-specificity and decreases intraspecific genetic variation in the symbiont (Frank 1996a). The suggested absence of fruiting bodies in the wild for the symbionts of Microtermes (Johnson et al. 1981, Darlington 1994) suggest a potential decrease in recombination and thus a decrease in genetic variation within the symbiont population. It may also increase host-specificity as vertically transmitted symbionts are less likely to part ways with the host as symbionts are harder to reobtain from the environment when sexual spores are absent. In chapter $\mathbf{6}$ I sampled different populations of Microtermes termites and their Termitomyces symbionts in Pretoria, South Africa. By sequencing a polymorphic marker for both the termites and their symbionts I showed that genetic diversity was high in both termites and fungal symbionts and that host-specificity was very low, indicating frequent horizontal exchange of symbionts between termite species.

The absence or rarity of mushrooms in certain species of Termitomyces is not unique for Microtermes symbionts. Cryptic diversity within Termitomyces has been proposed, since genetic variation does not 
align with the limited number of described species (Osiemo et al. 2010). Naturally occurring mushrooms for the symbiont of the termite $M$. natalensis have not been reported, although it is known to be able to fruit under artificial conditions (De Fine Licht, Andersen, and Aanen 2005, Vreeburg et al. 2020). The absence of mushrooms in some species of Termitomyces hampers the description of these species, and scientific communication. Describing species based on other diagnostic features than mushroom morphology such as wellcharacterized and publicly accessible cultures and molecular data has been proposed (Makonde et al. 2013). In chapter 7 I describe T. cryptogamus, the symbiont of the termite $M$. natalensis using this approach.

In chapter $\mathbf{8}$ I discuss the results of this thesis and discuss in more detail the origin of the symbiosis between termites and fungi and how these findings can improve our understanding of mutualistic symbioses in general. I also discuss parallels between termite fungiculture and human agriculture. Finally I give a more personal opinion on taxonomic challenges in the genus Termitomyces and how to resolve those. 


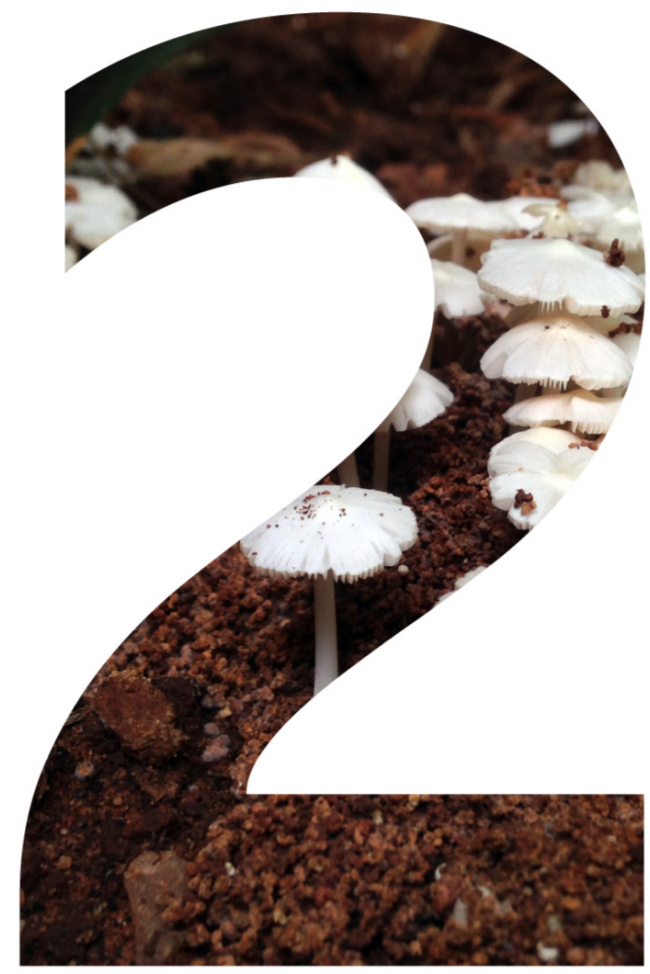

Ancestral predisposition toward a domesticated lifestyle in the termite-cultivated fungus Termitomyces

Lennart J.J. van de Peppel, Mathijs Nieuwenhuis Benjamin Auxier, Alexey A. Grum-Grzhimaylo Martha E. Cárdenas, Z. Wilhelm de Beer D. Jean Lodge, Matthew E. Smith Thomas W. Kuyper, Ana E. Franco-Molano Timothy J. Baroni, Duur K. Aanen

Published in Current Biology 


\section{Abstract}

The ancestor of termites relied on gut symbionts for degradation of plant material, an association that persists in all termite families (Bignell 2000, Chouvenc et al. 2021). However, the single lineage Macrotermitinae has additionally acquired a fungal symbiont that complements digestion of food outside the termite gut(Nobre and Aanen 2012). Phylogenetic analysis has shown that fungi grown by these termites form a monophyletic group - the genus Termitomyces, but the events leading towards domestication remain unclear (Aanen et al. 2002). To address this, we reconstructed the lifestyle of the common ancestor of Termitomyces using a combination of ecological data with a phylogenomic analysis of 21 related non-domesticated species and 25 species of Termitomyces. We show that the closely related genera Blastosporella and Arthromyces also contain insect-associated species. Furthermore, the genus Arthromyces produces asexual spores on the mycelium, which may facilitate their dispersal by insects when growing on aggregated subterranean faecal pellets of a plant-feeding insect. The sister relationship between Arthromyces and Termitomyces implies that insect-association and asexual sporulation, present in both genera, preceded the domestication of Termitomyces and did not follow domestication as has been proposed previously. Specialisation of the last common ancestor of these two genera on an insect-faecal substrate is further supported by similar carbohydrate-degrading profiles between Arthromyces and Termitomyces. We describe a set of traits which may have predisposed the ancestor of Termitomyces towards domestication, with each trait found scattered in related genera and species outside of the termite-domesticated clade. This pattern indicates that the origin of the termite-fungus symbiosis may not have required large-scale changes of the fungal partner. 


\section{Results \& Discussion}

\section{Phylogenetic relationships of the termitomycetoid clade}

The family Lyophyllaceae (Basidiomycota), to which Termitomyces belongs, harbours species with diverse ecologies, including saprotrophic, parasitic and mutualistic lifestyles, with frequent transitions between them (Hofstetter et al. 2014). The genus Termitomyces, which engages in a mutualistic symbiosis with termites, presents an enigma on how such an intricate symbiosis could have evolved. Previous studies were unable to confidently identify the origin of this symbiosis due to a limited number of phylogenetic markers in the analyses or because closely related taxa were not included in the datasets (Hofstetter et al. 2002, Matheny et al. 2006, Hofstetter et al. 2014, Bellanger et al. 2015). To reveal the origin of the termiteTermitomyces symbiosis, using field collections and herbarium material we collected 39 samples of 11 genera spanning Lyophyllaceae. We obtained whole-genome sequences from these samples and added another seven publicly available assemblies. We reconstructed the phylogeny of these 46 taxa using 1131 conserved nuclear genes (Figure 1A). The topologies generated by both coalescent-based ASTRAL analysis (Figure S1) and concatenation-based IQ-TREE analysis were in agreement. Therefore, we will focus on the IQ-TREE phylogeny for our discussion. As high marker numbers inflate bootstrap support (BS)(Kubatko and Degnan 2007), we additionally calculated gene and site concordance factors (gcf and scf, Figure 1A)(Ané et al. 2007, Minh, Hahn, and Lanfear 2020), which respectively show the fraction of gene trees or informative alignment sites supporting each node. Although concordance factors cannot be used to statistically test whether a node is well-supported by the data, they reflect the amount of ambiguity among loci for a given bifurcation.

Consistent with previous findings (Hofstetter et al. 2014, Bellanger et al. 2015), we recover a well-supported termitomycetoid clade, which consists of the genera Arthromyces, Blastosporella, Tephrocybe and Termitomyces (Figure 1). Sister to the termitomycetoid clade is the genus Myochromella, although there is conflict among gene trees as indicated by the low gene concordance factor (36\%). This indicates that the majority (64\%) of gene trees do not support the quartet composition of this node, but it is the quartet supported by the largest share of gene trees. The sister group of the termitomycetoidmyochromelloid group is the clade consisting of the genera Asterophora, Tricholomella, Sphagnurus and Lyophyllum. The position of the genus Calocybe remains unresolved as we did not recover significant bootstrap support for this split. Our phylogenomic analyses 
provide strong support for the hypothesis that the sister group of Termitomyces is the genus Arthromyces (100BS, $72.1 \%$ gcf, $37.7 \% \mathrm{scf}$ ) (Figure 1). The sister group of the Arthromyces-Termitomyces clade is the clade consisting of two Asian species; Tephrocybe sp. 4 and Tephrocybe sp. 5, supported by $100 \%$ bootstrap, but showing some discordance among gene trees (43.1\% gcf, 34\% scf). The sister group of the Arthromyces-Termitomyces-Tephrocybe sp. 4-Tephrocybe sp. 5 clade is the clade represented in our dataset by the European and North American $T$. rancida, Blastosporella zonata from central and south America, Tephrocybe sp. 2 from South America and sp. 3 from Asia (100BS, 37.6\% gcf, 33.1\% scf). The increased discordance for these branches is not surprising as their branch lengths are relatively short, indicating a rapid diversification event. The deepest split in the termitomycetoid clade is represented by Tephrocybe sp. 1 from Australia and this relationship is well supported (100BS, $90.7 \%$ gcf, $45.7 \%$ scf).

\section{Predispositions towards domestication in the ancestor of Termitomyces}

The evolutionary steps in the three main groups of fungus-insect symbiosis remain unclear. For fungus-growing beetles, the accepted hypothesis is that domestication was contingent on dispersal by the beetles partner, as the wild relatives of the cultivated fungi are also dispersed by insects (Harrington 1987, Harrington 2005). For fungusgrowing ants it is proposed that either insect-facilitated dispersal or mycophagy was the initial step (Mueller et al. 2005).The fungal partners in these two groups have multiple origins, and in the case of beetles also multiple origins of farmers, suggesting that fungal domestication in these groups was did not require many changes. In contrast, a single fungal lineage has been domesticated by a single group of termites (Aanen et al. 2002), which makes the mutualism between termites and fungi a 'singularity'. Such singularities can be interpreted as either difficult low-probability evolutionary events, or alternatively, that they are due to evolutionary priority effects, where first-movers suppress subsequent independent origins (De Duve 2005, Burnetti and Ratcliff 2020). The biological data we collected and our phylogenetic reconstruction allowed us to identify a set of five traits shared by Termitomyces and the non-termite-associated sister group Arthromyces: a carbohydrate-degrading profile with a reduced potential to degrade plant-cell wall components, a rooting stipe (pseudorhiza), the formation of asexual spores (conidia), an insectfaecal association and the loss of clamp connections (Figure 1). 
Strikingly, these traits are shared to varying degrees by other members of the termitomycetoid taxa, suggesting that termitomycetoid fungi have a predisposition to domestication. Furthermore, some of these traits are found outside the termitomycetoid clade, such as the conidiaproducing Asterophora or a (short) pseudorhiza in some specimens of Tricholomella (Buller 1924, Kalamees 1992). We hypothesize that the combination of these traits in the ancestor of Termitomyces allowed it to colonize the comb formed by the most recent common ancestor of fungus-growing termites.

\section{Reduced capacity for carbohydrate breakdown predates domestication}

Fungi use a broad set of secreted carbohydrate-active enzymes (CAZymes) to breakdown and metabolise carbohydrates outside of their hyphal bodies. The CAZyme profiles of a species correlate with their ecology(Floudas et al. 2012). Previous research showed that Termitomyces has a reduced complement for the breakdown of these substances (Poulsen et al. 2014), but the timing of this reduction, whether predating the termite symbiosis or not, remained an open question. To test whether this reduction occurred pre- or postdomestication, we assessed the predicted CAZymes of the taxa in our dataset (Data S1). On average we identified 219 CAZymes per taxon (maximum: 375, minimum: 144). Species of Termitomyces have 197 CAZymes on average $(\max 251, \min 144)$. The related non-termite associated taxa in the genera Arthromyces and Blastosporella have a slightly higher CAZyme complement, with 233 and 232 CAZymes, respectively. There is no clear pattern of change related to Termitomyces in all six functional CAZy classes (Figure 1A). Principal component analysis of a finer separation of CAZymes revealed that generally Termitomyces species cluster together, with Blastosporella and Arthromyces nearby, despite the large genetic distance between Termitomyces and Blastosporella (Figure 1C). Correcting the principal component analysis for phylogeny (Revell 2009) did not reveal any source of CAZyme variation from other members of the Lyophyllaceae (Figure S2E). Variation in CAZyme sets is unlikely to be related to sequencing quality, as we found no correlation between the number of reads used in the assemblies and the CAZyme, BUSCO, or the entire predicted proteome content (Figure S2A-D).

Analysis of the evolutionary history of the CAZyme gene families revealed no significant changes that could be ascribed to the transition to the termite symbiosis, which indicates that the reduced CAZyme complement predates the symbiosis (Figure 1). Five gene families of the 
119 total had evolutionary histories that were not explained by the phylogeny alone as tested using the CAFE analysis; AA1, AA3, AA9, $\mathrm{GH} 16$ and $\mathrm{GH} 5$. The changes in the evolutionary history of AA1 and AA3, both used in the oxidation of lignin, were based on increased gene family number in the two Tephrocybe rancida strains. The changes in the GH5 and AA9 families, both families involved in cellulose degradation, while significant, were restricted to individual species, with no internal nodes showing unexpected changes. The GH16 family, involved in breaking various $\beta$-1,3-glucan bonds, showed changes at the tips of the tree, as well as a reduction within Termitomyces.

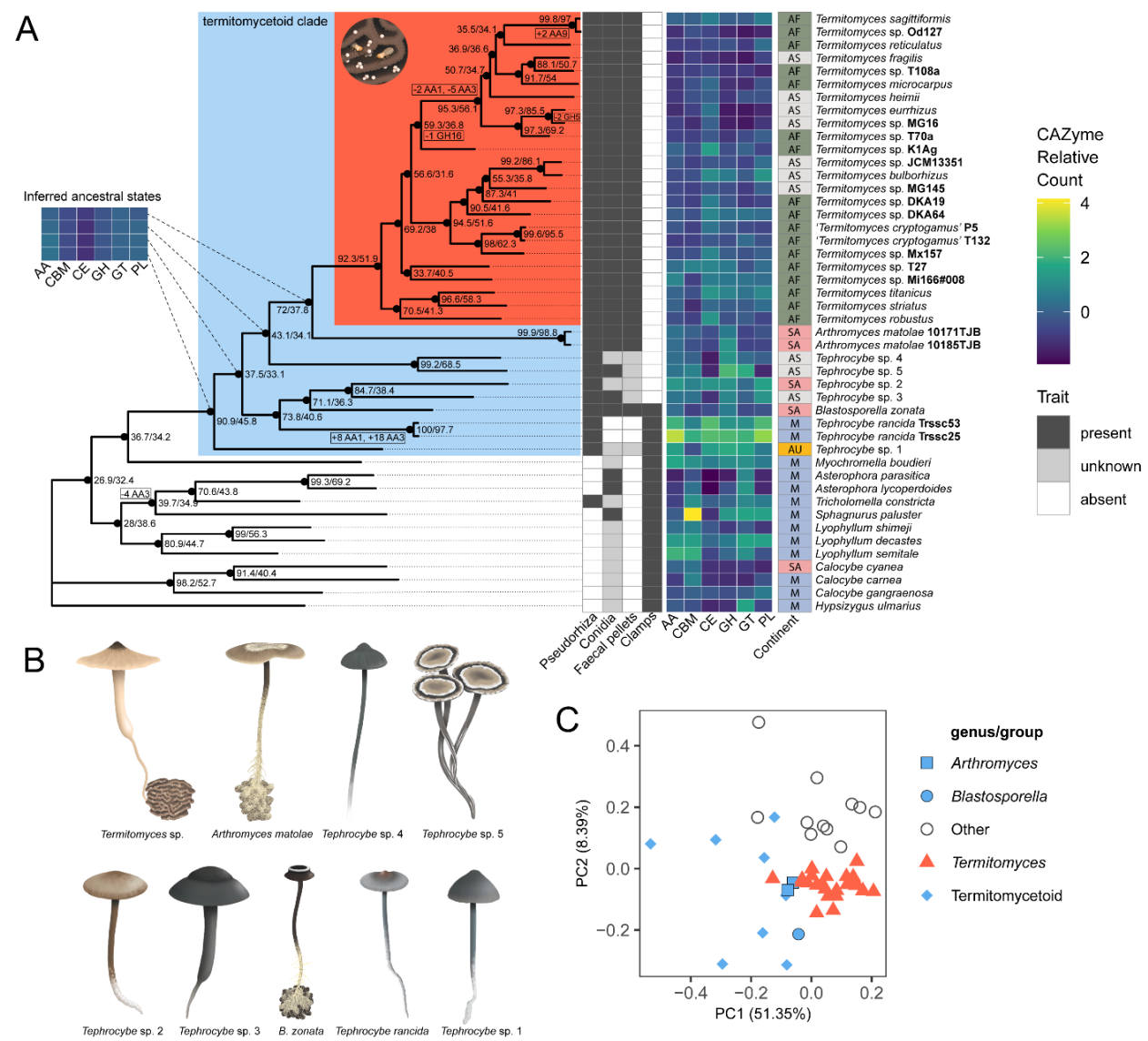

Figure 1 | Phylogenomic and CAZyme analysis of the Lyophyllaceae showing that the insect-associated Arthromyces is sister to the genus Termitomyces and showing a reduction in CAZyme complement prior to the symbiosis. A: Maximum-likelihood tree based on 1131 core nuclear (BUSCO) genes. Numbers at the nodes represent gene concordance factor/site concordance factor, dots indicate 100\% bootstrap support, 
numbers in boxes indicate significant changes in CAZymes. Hypsizygus ulmarius was used as an outgroup. Termitomycetoid clade highlighted in blue, termite-associated species highlighted in red. The most parsimonious reconstruction of the origin of the pseudorhiza is in the most recent common ancestor of the sister group of Tephrocybe sp. 1 as indicated. Matrix of morphological and CAZyme data: the left section of the matrix contains the character states of the predisposition traits: a pseudorhiza, production of asexual spores (conidia), an insect-faecal association, and the presence of hyphal clamp connections. The right section of the matrix shows a scaled heatmap of the total number of CAZymes split into the six main biochemical groups (Auxiliary Activity (AA), Carbohydrate Binding Module (CBM), Carbohydrate Esterase (CE), Glycoside Hydrolase (GH), Glycosyltransferase (GT) and Polysaccharide Lyase (PL). Colours indicate the difference of a taxon's CAZyme content from the average of the group, in standard deviations. CAZyme data of selected ancestral nodes inferred by CAFE is indicated to the left of the phylogeny, using the same colour scale as the main matrix. Significant changes in CAZymes not explained by the phylogeny are indicated in boxes next to the nodes. The column on the right indicates the continent on which each species occurs; Australia (AU), Africa (AF), Asia (AS), South America (SA) or multiple continents (M). B: drawings of representative species in the termitomycetoid clade showing their overall morphology and the presence and/or absence of the predisposition traits. C: Principal component analysis of the same CAZyme data as in the CAZyme matrix except that the six main groups are split into 119 CAZyme subfamilies, numbers in parentheses indicate the percentage of variance explained by a principal component. Note that samples of Termitomyces (red triangles) and Arthromyces (blue squares) cluster together.

\section{Insect-faecal associations predate domestication}

During field work, collections of $A$. claviformis, $A$. matolae and B. zonata showed that these species were associated with aggregated clumps of insect-faecal pellets in all cases (Figure 2). Faecal pellets upon which $A$. matolae was growing were composed of woody plant material (Figure S3). We were unable to identify the insect source of the pellets.

To identify the source of the faecal pellets in our $B$. zonata samples, we collected nearby abundant beetle larvae whose fresh faecal pellets were macroscopically similar to those found with $B$. zonata samples (Figure 2D). We used DNA barcode sequences to identify these as larvae of a Scarab beetle (Scarabaeidae), although we could not identify them to the species level, due to a lack of an identical or close matches in NCBI Genbank (STAR methods). Our novel finding of the insect-faecal pellet substrate of Arthromyces and B. zonata, raises the question whether other species in the termitomycetoid clade are also associated with insect faeces. We were unable to re-collect any of the species of 
Tephrocybe except T. rancida, but despite extensive efforts, we found no indication that $T$. rancida grows on insect faeces (STAR Methods and Figure S3). This apparent lack of an insect-faecal relationship is supported by the higher total number of CAZymes in the T. rancida genome compared to $B$. zonata (Data S1), including significant increases in AA1 and AA3 (Figure 1A). The phylogenetic position of the five unidentified species of Tephrocybe in the termitomycetoid clade, the pseudorhiza and their similarity in CAZyme profiles warrants recollection and detailed study of the substrate. It remains unclear if these taxa have unrecognized associations with insect faeces.

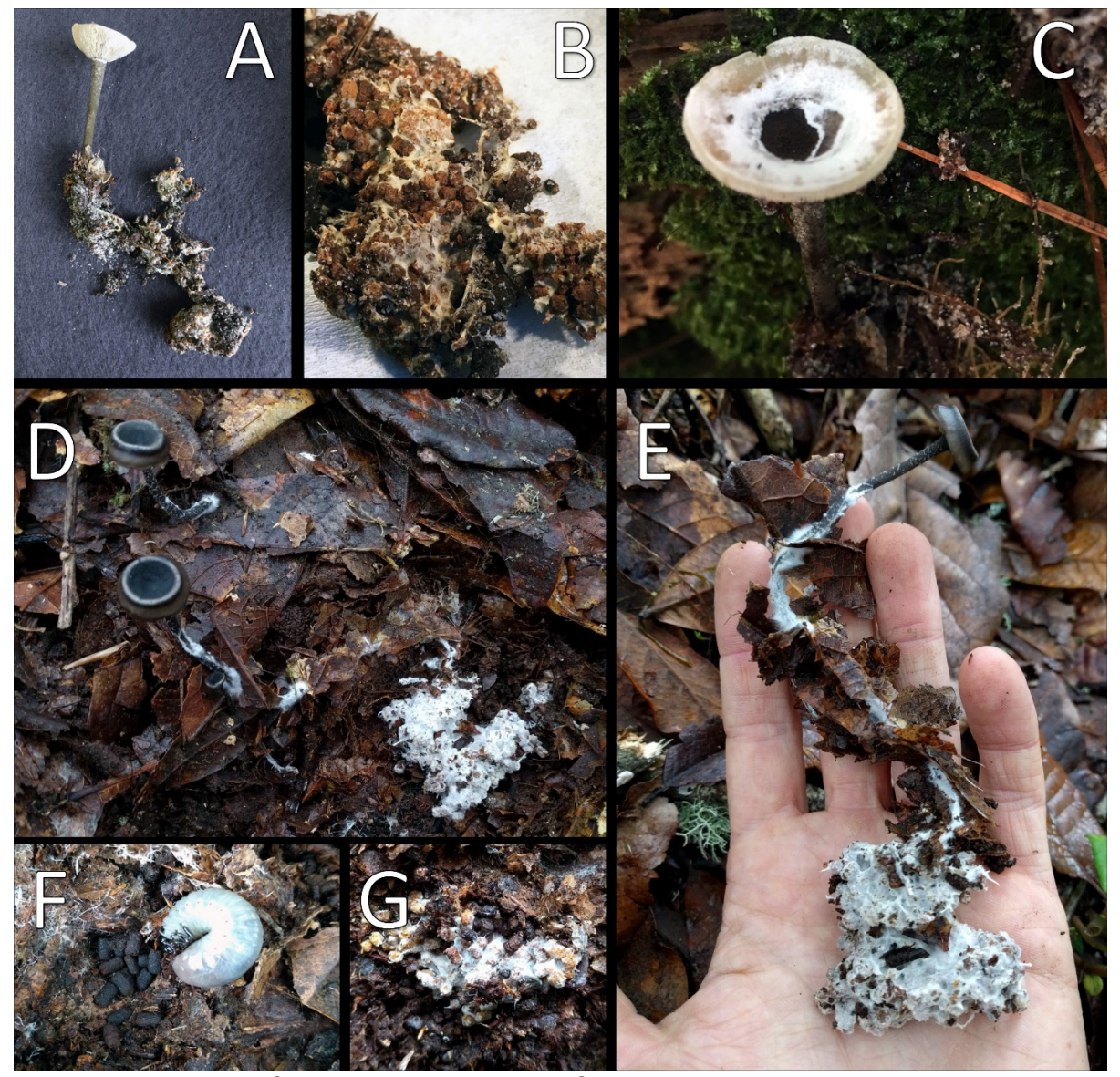

Figure 2 | Insect-faecal associations found in Arthromyces matolae and Blastosporella zonata. A: Mushroom of $A$. matolae connected to a faecal pellet mass. B: Close-up of the faecal pellets. C: Pileus of $A$. matolae showing the dark arthroconidia. D: B. zonata mushrooms and pellet mass. E: Detail of the mushroom of $B$. zonata showing the attachment with the pseudorhiza to the pellet substrate. F: Beetle larva next to fresh faecal pellets. G: Close-up of the faecal pellet mass showing white rot in the pellets. 


\section{Conidial production predates domestication}

While all mushroom-forming fungi produce sexual spores (basidiospores) for reproduction, only some species produce asexual spores (conidia), either on the mycelium or in rare cases on the mushroom (Kües et al. 2016). However, several species in the termitomycetoid clade, produce conidia (Figure 1). In species of Termitomyces only the mycelium produces conidia (Botha and Eicker 1991a) (Figure 3A-D), which are ingested by the termites and mixed in the gut to inoculate fresh fungus combs (Hinze, Crailsheim, and Leuthold 2002). These conidia are produced both inside termite mounds as well as when grown in laboratory culture. Previously, it has been reported that Arthromyces species produce conidial chains by fragmentation of terminal hyphae (arthroconidia) on the entire mushroom while $B$. zonata produces ornamented conidia in small spore heads by a budding process (so-called blastoconidia) on the mushroom cap (Baroni et al. 2007). Here, we report that $A$. matolae also produces dikaryotic conidia on the mycelium in laboratory culture (Figure $3 \mathrm{E}-\mathrm{H})($ Figure S4A). These conidia are encased in an elaborate structure composed of a hyphal net with large extended setae composed of single cells. We further found that $B$. zonata produces both dikaryotic blastoconidia and arthroconidia on the mycelium in culture (Figure S4BD). Arthroconidia were also detected on the mushrooms of Tephrocybe sp. 3 and Tephrocybe sp. 5. We did not find conidia in the following taxa: T. rancida, Tephrocybe sp. 1, Tephrocybe sp. 2 and Tephrocybe sp. 4. The current lack of laboratory cultures for the various Tephrocybe spp. prevents the confident pinpointing of the origin, or origins and subsequent losses, of conidial production in the termitomycetoid clade. However, the most parsimonious reconstruction is that the common ancestor of Arthromyces and Termitomyces produced conidia. 


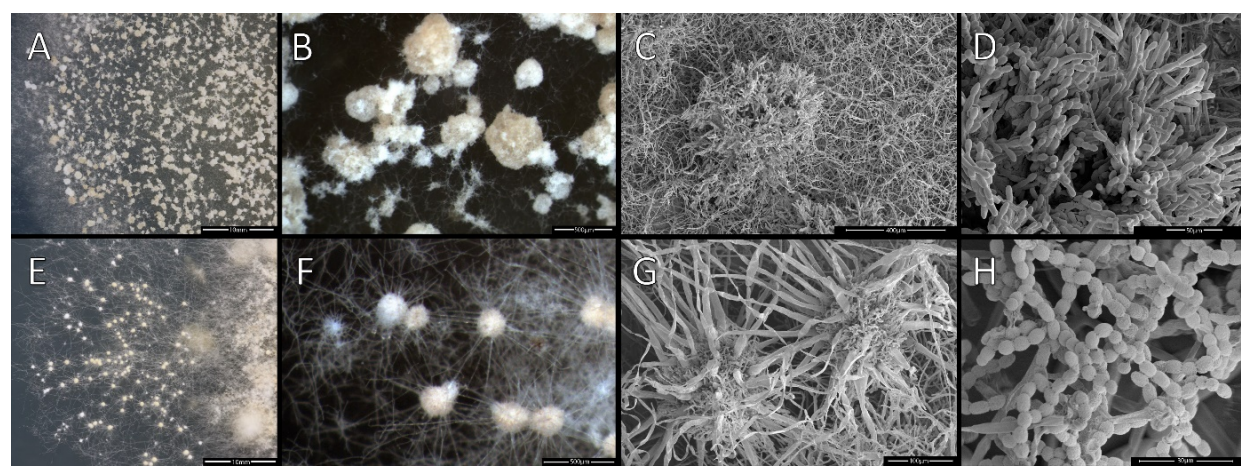

Figure 3 | Macroscopic and SEM microscopic images of the conidiophores of 'Termitomyces cryptogamus' and Arthromyces matolae. Conidiophores in laboratory culture of 'T. cryptogamus' (P5) (A,B). Electron microscope image of a conidiophore showing the chains of conidia (C). Close-up of a conidiophore showing the branched chains of conidia of ' $T$. cryptogamus' (D). Laboratory culture of $A$. matolae (FLAS-F-62734) showing the conidiophores $(E, F)$. Electron microscope image of $A$. matolae showing two conidiophores with large aerial setae (G). A close-up of an $A$. matolae conidiophore showing the chains of arthroconidia $(H)$.

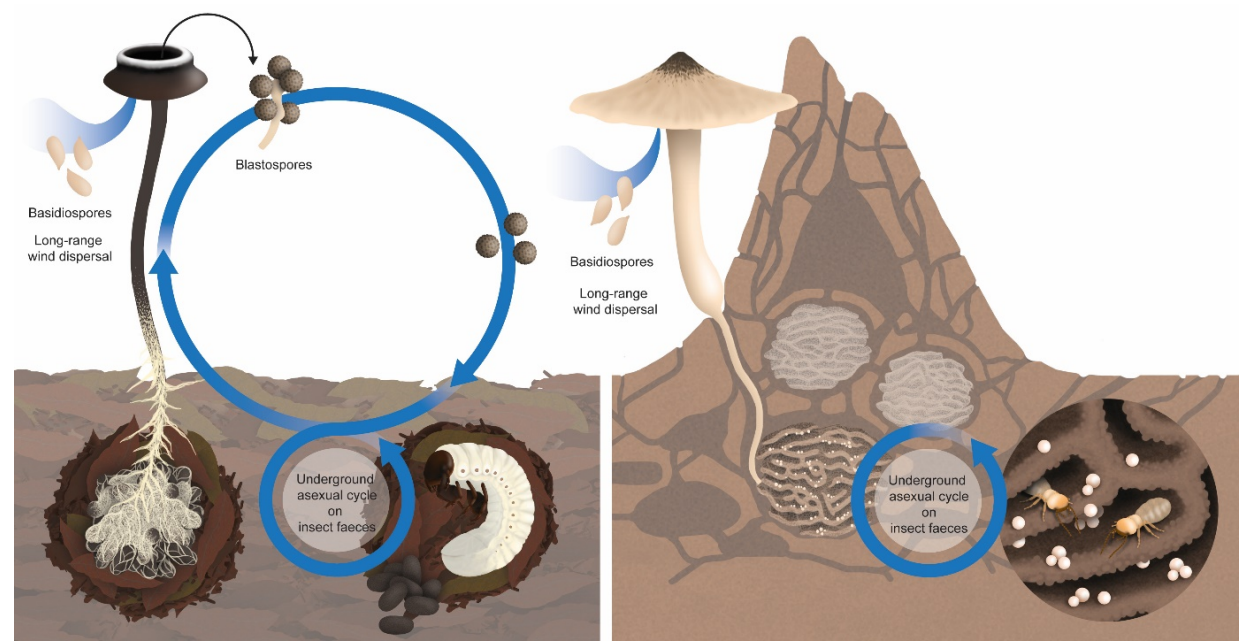

Figure 4 | Proposed insect-fungal interaction model of insect-faecal associated mushroom-forming fungi compared to the lifecycle of the domesticated genus Termitomyces. In the aboveground part, asexual spores produced on the mushroom are used for local dispersal and rapid inoculation of the faecal pellets. In the belowground part, asexual spores may stick to an insect and inoculate fresh batches of faecal pellets. Additionally, the conidia could also facilitate faster colonization by reinoculation of the substrate via the insect host. Wind-dispersed basidiospores are used for long-range dispersal and sexual recombination. 


\section{A rooting stipe predates domestication}

All species within the termitomycetoid clade are able to produce a rooting stipe (pseudorhiza). Within Termitomyces, T. microcarpus may only produce a tiny pseudorhiza in some cases, probably as a response to epigeous fruiting on expelled comb material (Horak 1968). The pseudorhiza is a specialized part of the stipe which pushes the immature mushroom of the fungus from the buried subterranean nutrient substrate to the soil surface (Buller 1934) and has evolved independently several times in Basidiomycota. The nutrient substrate can be a tree root in the case of Phaeocollybia and Xerula radicata (Campbell 1938, Redhead and Malloch 1986), a wood mouse or mole latrine in the case of Hebeloma radicosum (Sagara et al. 2000b, Sagara, Senn-Irlet, and Marstad 2006), and insect faeces in the case of Arthromyces, B. zonata (Figure 2) and Termitomyces, where insect faeces form a specialised fungus comb. All termitomycetoid species are able to produce a pseudorhiza (Figure 1) which indicates a single transition towards growth on a buried nutrient substrate in this group.

\section{Loss of clamp connections predates domestication}

There is a striking pattern in a range of mutualisms with convergent increases in genome copy numbers per cell, either through polyploidy or multiple nuclei (Nobre et al. 2014). Examples are the multinucleate cells of the fungi cultivated by leaf-cutting ants (Kooij et al. 2015), the fungi forming arbuscular mycorrhizae with plants (Kuhn, Hijri, and Sanders 2001), the polyploid endosymbiotic plastids and mitochondria of eukaryotic cells (Bendich 1987), and even our own domesticated crops, most of which are polyploids (Bretagnolle and Thompson 1995). Termitomyces also fits in this pattern with multinucleate cells containing up to 10 nuclei, lacking the specialised morphological structures, clamp connections, that maintains nuclei per cell in most other basidiomycete fungi (De Fine Licht, Andersen, and Aanen 2005, Nobre et al. 2014). This striking similarity suggests that increased ploidy of symbionts may be selected as a consequence of a symbiotic lifestyle. However, the most parsimonious reconstruction is that clamp connections were lost before domestication of Termitomyces since its sister group Arthromyces also lacks clamp connections (Baroni et al. 2007). The origin of multinucleate cells without clamp connections presumably is even older, predating the split between the Termitomyces-Arthromyces clade and the Tephrocybe sp. 4 and sp. 5 clade, but following the second split in the termitomycetoid clade, between Tephrocybe sp. 1, which has clamp connections, and its sister group, most members of which lack clamp connections. 


\section{Origin of termite-fungus symbiosis}

Three pathways have been described to explain the evolution of a domestication interaction; commensal, prey-or-harvest, and direct (Zeder 2012, 2015). The latter two primarily apply to human domesticators since they involve conscious selection of a potential domesticate. In the commensal pathway, a two-way partnership can arise if species $A$ enters the habitat of species $B$ where it can make use of a niche provided by species B (Zeder 2015). Previously, the sister group of Termitomyces and its biology have remained unknown, preventing identification of this potential niche. The consumption of fungus-infested wood has been shown to increase survival in several different species of termites (Viana et al. 2018). Some species of termites are also known to consume mushrooms (Waller et al. 1987) and fungus-growing termites have been observed to consume mushrooms other than Termitomyces (Chiu et al. 2019). The observation that termites consume and are attracted to rotting wood led to the hypothesis that mycophagy was the initial step towards domestication (Batra and Batra 1979, Rouland-Lefèvre 2000, Mueller et al. 2005). This explanation may be too simplistic as it does not recognize a potential niche provided by the termites that could be utilized by the fungus. Our novel findings on the biology of species in the termitomycetoid clade suggests that the ancestor was not a generalist wood degrader but already possessed a suite of traits which predisposed it towards domestication. This is unique among the fungus-growing insects as ancestral predispositions have not been documented in fungusgrowing ants and ambrosia beetles.

Our phylogenetic analysis provides strong support for a sistergroup relationship between Arthromyces and Termitomyces. Both genera share the five traits discussed above, so the most parsimonious reconstruction is that the common ancestor of Termitomyces grew on insect faeces and had the carbohydrate-degrading enzymatic profile to utilize that growth substrate, produced conidia, had multinucleate cells with no clamp connections and had a rooting stipe. Our results therefore imply that an insect-faecal association predated the termitefungus symbiosis. This insect-faecal association may even predate the common ancestor of Arthromyces and Termitomyces as several other taxa in the termitomycetoid clade exhibit various combinations of these five traits.

All non-Termitid termite families rely on cellulolytic protist gut symbionts for the digestion of wood (Brugerolle and Radek 2006). However, these gut symbionts have been lost in the Termitidae (Darlington 1994, Eggleton 2006). The loss of gut symbionts was 
correlated with a diversification of feeding habits, including fungusfarming, bacteria-farming and soil-feeding (Inward, Vogler, and Eggleton 2007, Chouvenc et al. 2021). Recent evidence suggests that the subfamily Sphaerotermitinae, the only extant member of which constructs combs similar to fungus-growing termites but are instead colonized by bacteria, is the sister group of the fungus-growing termites (Garnier-Sillam et al. 1989, Bucek et al. 2019). The most parsimonious reconstruction for comb evolution is that comb-building was present in the common ancestor of the Sphaerotermitinae and the fungusgrowing termites (Bucek et al. 2019). After the loss of the flagellated protists, the ancestral comb may have served as an "external rumen" in fungus-growing termites (Nobre and Aanen 2012), providing a suitable substrate for a fungus adapted to grow and reproduce on a similar faecal substrate. As wood-decay fungi are more efficient lignin degraders compared to lignocellulolytic bacteria (Sigoillot et al. 2012), the increased capacity to degrade lignin and cellulose combined with a reduced capacity to break down oligosaccharides of the ancestors of Termitomyces may have increased the amount of useable calories in the comb substrate which directly benefitted the termites. Given the sistergroup relationship between Arthromyces and Termitomyces, the most parsimonious reconstruction is that the appendiculate conidiophores formed by Arthromyces and the nodules of Termitomyces are homologous. The conidia may have served an important role in maintaining the fungus as the dominant species within the ancestral comb since the conidia could facilitate local dispersal and continuous substrate reinoculation (Figure 4).

The conidia produced on the mushroom in Arthromyces and several other species in the termitomycetoid clade may be involved in local dispersal since they are thick-walled and this likely increases their chances of survival during periods when substrate is unavailable. This strategy has been suggested for the mycoparasitic genus Asterophora as well because their hosts are only available seasonally and patchily (Buller 1924, Redhead et al. 1994). It seems likely that they serve the purpose of survival structures such as chlamydospores or sclerotia that are found in a wide array of Basidiomycota (Smith, Henkel, and Rollins 2015, Kües et al. 2016).

According to our hypothesis, the niche provided by the termites for the ancestor of Termitomyces was the pre-digested plant material in the ancestral comb. Besides mineral nutrients obtained from consuming the fungus, the termites could also benefit from the increase in available calories of the comb as a result of fungal degradation and the oligosaccharides left behind by the fungus. The fungus benefitted 
from a physically pre-treated substrate with an enlarged surface area combined with local dispersal and reinoculation by the termites.

Non-wind dispersed asexual spores (produced by the fungus) that are locally dispersed by a different species (the termites) is analogous with human domestication and propagation of food plants. It has been proposed that the reduction of natural seed dispersal, which includes the loss of seed shattering, is a key trait selected for under cultivation (Diamond and Ordunio 1999, Purugganan and Fuller 2009). Most seed crops have non-shattering seeds, which renders a plant species with limited dispersal abilities and primarily dependent on humans for survival and propagation (Diamond 2002). Similarly, Termitomyces depends on termites for substrate colonisation and accordingly for survival and propagation (Leuthold, Badertscher, and Imboden 1989). The finding that the sister group of Termitomyces produces conidia and is associated with insect faeces lends support to the hypothesis that one of the first steps in the domestication process was local dispersal by insects.

\section{Acknowledgements}

We would like to thank the Colombian students from the University of Antioquia who assisted with collecting $B$. zonata during fieldwork. We thank Dirk Stubbe for providing a specimen of Tephrocybe sp. 5 We thank Marc Maas for creating Figure 4. We thank the Royal Botanical Gardens KEW herbarium for letting us use their facilities and providing specimens. We thank the field mycologists who helped us with taxon sampling; Carolien Reindertsen, Mirjam Veerkamp, Arthur Grupe, Jenny Rogers, Jacob Kalichman and Gert Immerzeel. We thank Natascha Oosterwijk for making some of the microscopic images. We thank Marcel Giesbers from the Wageningen Electron Microscopy Centre for his help in making the electron microscopy images. DKA, LJJvdP, MN, AAGG and BA were supported by the Netherlands Organisation for Scientific Research (DKA, LJJvdP, MN, and AAGG by VICI: NWO 86514007; DKA and BA by ALWGR.2017.010). TJB and DJL acknowledge grants from NSF (DEB-9525902 and DEB-0103621 Belize, Puerto Rico, Dominican Republic), TJB for support from the National Geographic Society (Belize) and the New York Botanical Gardens (Thailand and Australia) supporting field research that allowed discovery of many of the novel non-Termitomyces taxa included in this study.

The Colombian biological material used in this work was collected under the permission given to the University of Antioquia 
according to resolution 0524 of May 27, 2014 issued by the National Environmental Licensing Authority - ANLA.

\section{Author contributions}

Conceptualization, LJJvdP, DKA; Methodology, LJJvdP, DKA, MN, BA; Investigation, LJJvdP, DKA, MN, AAGG, TJB, MES, MEC AEFM, TWK, ZWdB; Formal analysis; LJJvdP, MN, BA; Resources, TJB, MES, MEC, AEFM, DJL, TWK; Data Curation, MN; Writing - Original Draft, LJJvdP. All of the authors commented on the first draft and approved the final version of the manuscript.

\section{Declaration of interests}

The authors declare no competing interests.

\section{STAR $\star$ Methods}

\section{RESOURCE AVAILABILITY}

\section{Lead contact}

Further information and requests for resources should be directed to and will be fulfilled by the Lead Contact, Lennart van de Peppel (lennartvdpeppel@gmail.com)

\section{Materials availability}

A culture of Arthromyces matolae (FLAS-F-62734) was submitted to the culture collection of the Westerdijk Fungal Biodiversity Institute under the accession number CBS 147616.

\section{Data and code availability}

Newly generated raw reads of the fungal taxa are available in the Sequence Read Archive (SRA) with accession numbers: SRX10313000SRX10313007, SRX10337354-SRX10337371 and SRX4910404SRX4910415. Assembled genomes are available at NCBI Genbank with accession numbers: GCA_017580835.1, GCA_017607575.1, GCA_017657195.1, GCA_017657225.1, GCA_017657235.1, GCA_017657275.1, GCA_017657295.1, GCA_017657335.1, GCA_017657355.1, GCA_017657315.1, GCA_018220975.1, GCA_018221615.1, GCA_018221655.1, GCA_018221735.1, GCA_017657375.1, GCA_018221635.1, GCA_018221805.1, GCA_018282005.1, GCA_018221785.1, GCA_018849495.1, GCA_018850235.1， GCA_018282025.1، GCA_018850275.1, GCA_018850815.1, GCA_018850255.1, GCA_018851285.1, 
GCA_018851305.1,

GCA_018851325.1,

GCA_018851835.1,

GCA_018854895.1,

GCA_018855395.1,

GCA_018855915.1,

GCA_018856295.1,

GCA_018857265.1,

GCA_018857285.1,

GCA_018857305.1, GCA_018858115.1. Beetle larva sequences are available at NCBI Genbank with accession numbers: MW698941 and MW698942.

Scripts used for filtering contigs and removing bacterial contamination from the assemblies in the bioinformatic analyses as well as the scripts used in the CAZyme analysis are available at: https://github.com/BenAuxier/Termite.Domestication

\section{EXPERIMENTAL MODEL AND SUBJECT DETAILS}

We sampled a total of 47 taxa, of which 25 were Termitomyces, across the entire family of the Lyophyllaceae and within the Lyophyllaceae we focused primarily on the 'termitomycetoid' clade (Hofstetter et al. 2014). For the three remaining clades we sampled one to four representative species. We collected specimens from various sources; the Termitomyces samples were obtained from our in-house culture collection, the Royal Botanical Gardens KEW herbarium and from mushrooms stored in ethanol collected in Ivory Coast. Other nonTermitomyces lyophylloid herbarium specimens were obtained from the Royal Botanical Gardens KEW herbarium and the Cort herbarium. Fresh Lyophylloid mushrooms were provided by various collectors from the Netherlands, DNA was isolated either directly from these mushrooms or from tissue cultures. Tissue cultures were made by cutting the stipe or pileus and moving a sterile piece of tissue from the inside of the stipe or pileus with sterile forceps into a petri dish containing malt yeast extract agar (per litre demi water: $20 \mathrm{~g}$ malt extract, $2 \mathrm{~g}$ yeast extract, $15 \mathrm{~g}$ agar) and streptomycin $(30 \mathrm{mg} / \mathrm{L})$ against bacterial contamination. A full overview of all samples can be found in Data S2.

\section{METHOD DETAILS}

\section{Detection and collection of faecal pellets}

Mushrooms of Blastosporella zonata and pellets were collected in Murillo - Tolima, Colombia. Between ten and 15 pellets were stored in $1.5 \mathrm{ml}$ Eppendorf tubes with pure ethanol for subsequent analysis. In some cases, pellets were found very close to the pellet mass on which $B$. zonata was growing. These pellets, which did not show fungal colonization, were collected separately. 
On two occasions (collection Bzo6 and Bzo8) scarabid beetle larvae were found in close proximity of the pellet substrate. A total of six larvae were collected, three larvae were found within a $15 \mathrm{~cm}$ radius of collection Bzo6 and one larva was found within the same radius of collection Bzo8. Two additional larvae were collected randomly in leaf litter.

The larvae were collected in $50 \mathrm{ml}$ tubes to collect fresh faecal pellets. Fresh pellets and beetle larvae were stored in pure ethanol. Fresh fruiting bodies were stored in $50 \mathrm{ml}$ tubes and tissue cultures were made on the same day. Conidial cultures were made by streaking conidia from the pileus of the fruiting body on a petri dish containing the same medium. Cultures were stored at room temperature.

To detect potential faecal pellets and to study the function of the pseudorhiza of Tephrocybe rancida, we sampled mushrooms at one location on the property of the Nyenrode Business University in Breukelen, the Netherlands. We sampled in late October of 2015, 2017 and 2019. The rooting base was carefully excavated using a small gardening trowel. The pseudorhiza was traced into the soil but no clear connection to faecal pellets or any buried substrate could be found. Fruiting bodies were collected in $50 \mathrm{ml}$ tubes and were either dried or used to make tissue cultures. Single-spore isolates were made by attaching a pileus with petroleum jelly to the lid of a petri dish and spores were captured on MYA medium with streptomycin. When basidiospores had germinated a single colony was transferred to a new MYA plate.

\section{Scoring of the morphological predisposition traits}

The four morphological traits were scored after inspection of the specimens that were collected. In most cases the traits could unambiguously be detected from our collections. However, in some cases we were not able to score all four traits as we only had a culture and no mushroom or the other way around. In these cases, scoring was done combining our personal observations and those from literature. Our single collection of Tricholomella constricta did not have a pseudorhiza; however, this trait is variable within this species (Kalamees 1992), and therefore, we scored it as 'present'. In the case of Termitomyces we made the general assumption that all species have a pseudorhiza although there may be a single case in which there may not always be a pseudorhiza, which is in T. microcarpus (Heim 1977). However, because some authors describe it as weakly rooting (Horak 1968), it is probably still able to produce the pseudorhiza and we therefore scored it as 'present'. The ambiguity of this trait in $T$. 
microcarpus is most likely a response to epigeous fruiting on expelled comb material.

We were not able to detect conidia in our culture of Sphagnurus paluster; however, conidial production has been reported for this species (Walther, Garnica, and Weiss 2005) and we therefore scored it as 'present'.

The presence of clamp connections has been reported in the following genera or species: Asterophora (Thompson 1936), Blastosporella (Baroni et al. 2007), Calocybe cyanea (Baroni et al. 1999) and Hypsizygus (Singer 1947), Lyophyllum (Moncalvo, Rehner, and Vilgalys 1993), Myochromella (Hofstetter et al. 2014), Tricholomella (Kalamees 1992), T. rancida (Hofstetter et al. 2014), S. paluster (Redhead 1981). The absence of clamp connections is reported in the following genera: Arthromyces (Baroni et al. 2007) and Termitomyces (Heim 1977, Nobre et al. 2014).

\section{DNA isolation}

For DNA isolation of the fungal samples a small piece $(0.2-0.5 \mathrm{~g})$ of mycelium from a laboratory culture, the pileus of a dried herbarium specimen or the pileus of a specimen stored in ethanol was frozen in liquid nitrogen and disrupted in a $1.5 \mathrm{ml}$ Eppendorf tube with glass beads prior to DNA isolation. DNA isolation for all samples was performed using the cetyltrimethylammonium bromide (CTAB) as previously described (Nieuwenhuis et al. 2019). DNA from beetle larvae was isolated from a leg part using the same protocol.

DNA from faecal pellets was isolated by using a Nucleospin Soil DNA extraction kit (Macherey-Nagel) following the manufacturer's instructions. For the fresh pellets that were directly obtained from living beetle larvae only a single pellet was used for DNA isolation, from the pellets that were part of the fungal substrate between five and 20 pellets were used depending on the size of the pellets and availability.

\section{Beetle larvae identification}

As the identification of beetles from the larval stage is very difficult and requires a field expert, we attempted molecular identification of the six larvae that we collected. This was done by obtaining a partial sequence of the mitochondrial cytochrome $c$ oxidase (CO1) and using NCBI BLAST for identification. A partial sequence of the $\mathrm{CO} 1$ was amplified using the primer pair C1-J-2183 ('Jerry')/ TL2-N-3014 ('Pat')(Simon et al. 1994), using the following PCR program: denaturation at $94^{\circ} \mathrm{C}$ for 60 seconds, then five cycles consisting of 30 seconds denaturation at $94^{\circ} \mathrm{C}$, 40 seconds annealing at $47^{\circ} \mathrm{C}$ and elongation for 60 seconds at $72^{\circ} \mathrm{C}$, 
followed by 30 cycles consisting of 30 seconds denaturation at $94^{\circ} \mathrm{C}, 40$ seconds annealing at $52^{\circ} \mathrm{C}$ and elongation for 60 seconds at $72^{\circ} \mathrm{C}$, followed by a final extension step for 10 minutes at $72^{\circ} \mathrm{C}$. After Sanger sequencing of amplified products, we were able to distinguish two different genotypes, of which four larvae with identical sequences were of genotype 1 (MW698941) and two larvae with identical sequences of genotype 2 (MW698942). We could not make a reliable identification for either genotype because searches against the Genbank database did not return a close match (97\% similarity). The closest match for genotype 1 was a $85.75 \%$ match to a Cryptodus sp. sequence (KF801857), while genotype 2 had a $84.17 \%$ match to a Pimelopus dubius dubius sequence (EF487738). Both of these species belong to the subfamily Dynastinae (Rhinoceros beetles) within the Scarabaeidae.

\section{Faecal pellet identification}

Identification of the depositor of the faecal pellets of B. zonata or Arthromyces could shed light on the interaction between the fungus and the insect partner. As we were only able to obtain fresh pellets in ethanol for $B$. zonata we focused on these pellets. We attempted amplification of the mitochondrial cytochrome coxidase (CO1) on DNA extracted from pellets using PCR with primer pairs, LCO1490/HCO2198 (Folmer et al. 1994) and Jerry/Pat (Simon et al. 1994). We also attempted amplification of the internal transcribed spacer 1 (ITS1) marker using the Vogler primer pair (Vogler and DeSalle 1994) and the 16S marker using the Coleoptera specific primers Coleop_16Sc and Coleop_16Sd (Epp et al. 2012). PCR was performed using protocols and conditions described for each primer pair specified in the original publications. We could not confidently observe amplification of host DNA as we obtained multiple different PCR products per reaction.

\section{Environmental DNA barcoding}

Our standard PCR protocol was insufficient to identify the depositor of the pellets so we opted for an environmental DNA barcoding approach. A recent study used environmental DNA barcoding approaches to determine dietary arthropod contents in faecal samples of insectivorous animals (Jusino et al. 2019). We used two different DNA barcodes: a $157 \mathrm{bp}$ target region of the CO1 using the primer pair ZBJ-ArtF1c/ZBJArtR2c (Zeale et al. 2011) and a 156bp target region of $16 \mathrm{~S}$ using the primer pair Ins16S_1shortF/Ins16S_1shortR (Clarke et al. 2014). PCR was performed using protocols and conditions described for each primer pair specified in the original publications. A total of 19 PCR reactions were done using the protocol below on 13 different DNA 
samples; six from a B. zonata pellet substrate, five from pellets not visibly colonized by $B$. zonata and two from fresh beetle pellets. Unique barcode adapters were used for each PCR reaction and all samples were pooled after PCR and sequenced using an Oxford nanopore MinION. To test whether the pellets were of beetle origin we used the sequences generated from the larvae that we collected as reference. We used Geneious 10.0.9 (www.geneious.com) to match reads (between 1,000 and 27,000 reads per sample) against the reference but were unable to find any significant matches (data not shown)(Kearse et al. 2012). Since we used two DNA samples from faecal pellets directly obtained from these larvae this suggests that our method may not be sensitive enough to pick up host DNA (from gut epithelial cells) from the faecal pellets.

\section{Electron microscopy}

Scanning electron microscopy on laboratory cultures of $B$. zonata (Bzo9), A. matolae (FLAS-F-62734) and 'T. cryptogamus' (P5) was performed at the Wageningen Electron Microscopy Centre. To preserve the delicate conidiophores in $A$. matolae and the nodules in 'T. cryptogamus' samples were frozen in liquid nitrogen prior to imaging (cryoSEM). The A. matolae culture that was used was grown for 14 days at $15^{\circ} \mathrm{C}$ on MYA agar. The 'T. cryptogamus' culture was grown for 25 days at $25^{\circ} \mathrm{C}$ on MYA.

\section{Library preparation and whole genome sequencing}

Library preparation and whole-genome sequencing was performed by Novogene (Hong Kong) using the Illumina Hiseq 2500 platform. The paired-end reads that were generated were $150 \mathrm{bp}$ long and the insert size was $500 \mathrm{bp}$.

\section{Assembly and annotation}

We assembled paired Illumina reads using SPAdes v.3.5.0 with default settings (Nurk et al. 2013). Short contigs smaller than $300 \mathrm{bp}$ or contigs with a coverage lower than $5 x$ were removed from the assembly using a script. Presumed bacterial contigs were removed from the assembly using a script which matched contigs using BLAST against a reference library of 500 randomly selected bacterial genomes. Contigs with a BLAST hit with an expect value (E) of less than 1e-7 were removed from the assembly. We applied automatic annotations to each assembly using the funannotate pipeline (v.1.7.4). We ran funannotate -mask with default options for repeat masking, followed by funannotate -sort. We then ran funannotate -predict using a pretrained Augustus data set for 
'Termitomyces cryptogamus' T132 as reference (Stanke and Morgenstern 2005). We also ran funannotate -iprscan, funannotate remote with antiSmash, and funannotate -annotate all with default settings(Palmer 2019).

\section{Marker selection and phylogenetic analysis}

We collected conserved orthologs using BUSCO with the basidiomycete reference gene set odb9 provided on the BUSCO website (Seppey, Manni, and Zdobnov 2019). We then removed any sequence sets for which we found fewer than 25 matches. Finally, we aligned the remaining sequence sets using MAFFT v.7.475 (Katoh and Standley 2013) with the following parameters: --auto --maxiterate 1000 --adjustdirection

To remove poorly aligned regions, we used Gblocks v. $0.91 \mathrm{~b}$ (Castresana 2000) with the following input: $-0-b 5=h-t=D N A$.

We then concatenated all trimmed alignments and ran a maximum likelihood phylogenetic analysis with IQ-TREE (version 2.1.2) with the following settings: -s -spp -o -bb 1000 -bsam GENESITE -m TESTMERGE --runs 100, with a partition for each BUSCO locus, and Hypsizygus ulmarius as outgroup. Using -bsam GENESITE reduces bootstrap inflation by resampling partitions first and then resampling sites within partitions. We ran 100 independent runs and all produced the same topology with minimal variation in likelihood estimates. To estimate phylogenetic conflicts between loci we compared species tree to individual locus trees estimated with IQ-TREE (version 2.1.2)(Minh et al. 2020). We reconstructed the locus trees with the following parameters: -s -S. The gene and site concordance factors were then computed with IQTree using the command: - $t$--gcf-s --scf 100. In addition, we used the locus trees generated by IQ-TREE to perform a coalescent-based species tree reconstruction using ASTRAL(Mirarab et al. 2014). We performed ASTRAL with default settings using the command: java -jar astral.5.6.3.jar -i -o 2>out.log.

\section{CAZyme analysis}

Predicted CAZymes were collected from the funannotate output for each assembly in our dataset. These predictions are made from the dbCAN2 database based on Hidden Markov Model predictions. To increase the confidence of the predictions, we then submitted the corresponding amino acid sequences to the dbCAN2 webserver, to obtain the predictions for this set of proteins using DIAMOND and Hotpep (Zhang et al. 2018). Only predicted CAZymes that were also 
identified using either the DIAMOND or Hotpep pipelines were used for the analysis.

\section{CAFE analysis}

To detect significant changes in CAZyme composition along our phylogenetic tree we ran CAFE (De Bie et al. 2006) on our CAZyme dataset using default settings. The phylogenetic tree reconstructed using IQ-tree was made ultrametric using the package Ape in $\mathrm{R}$ and was used as input for the analysis (Paradis and Schliep 2019).

\section{Supplemental materials}

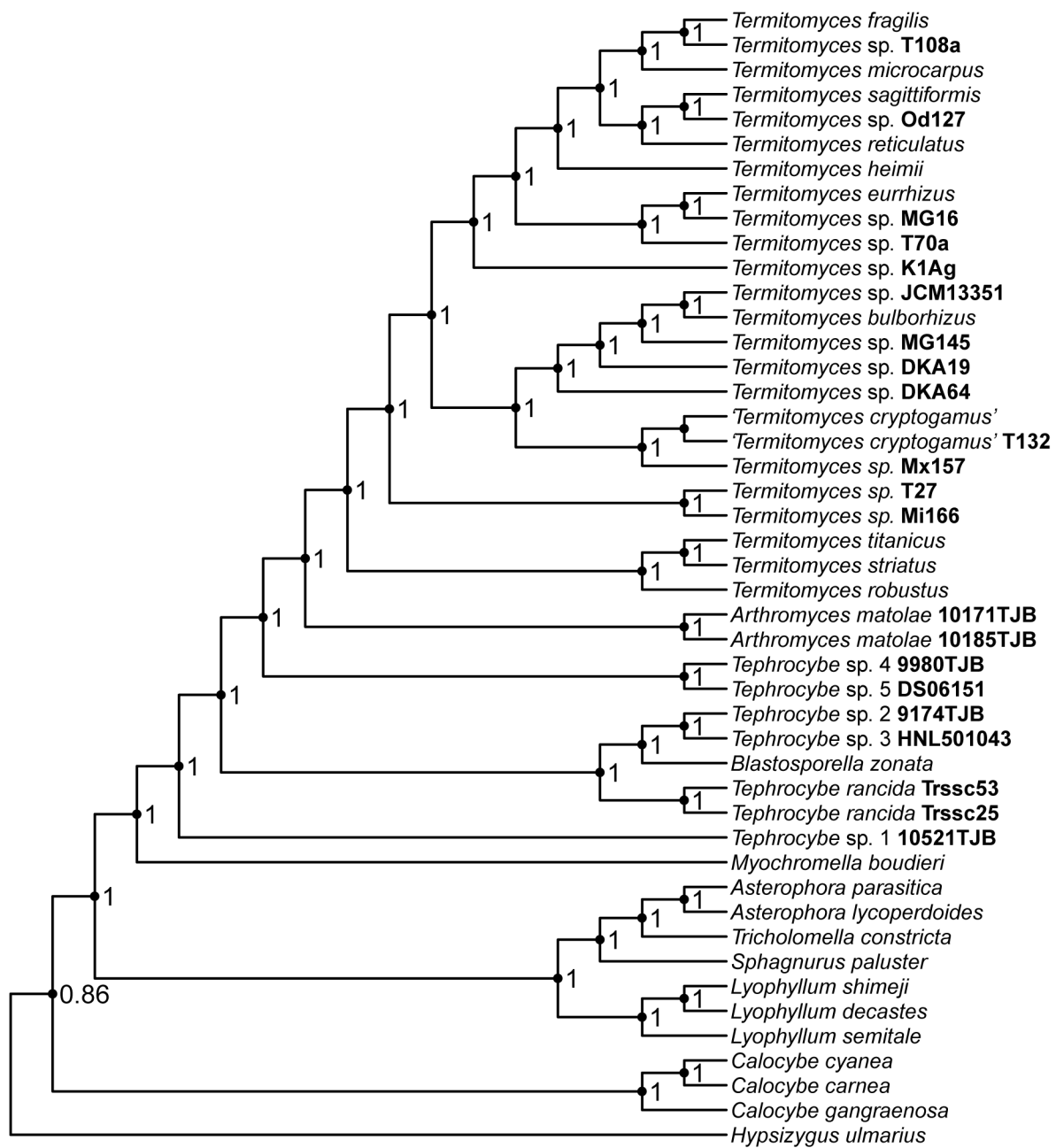

Figure S1 | Phylogenetic tree resulting from coalescent-based ASTRAL analysis based on the same dataset used for Figure 1. 


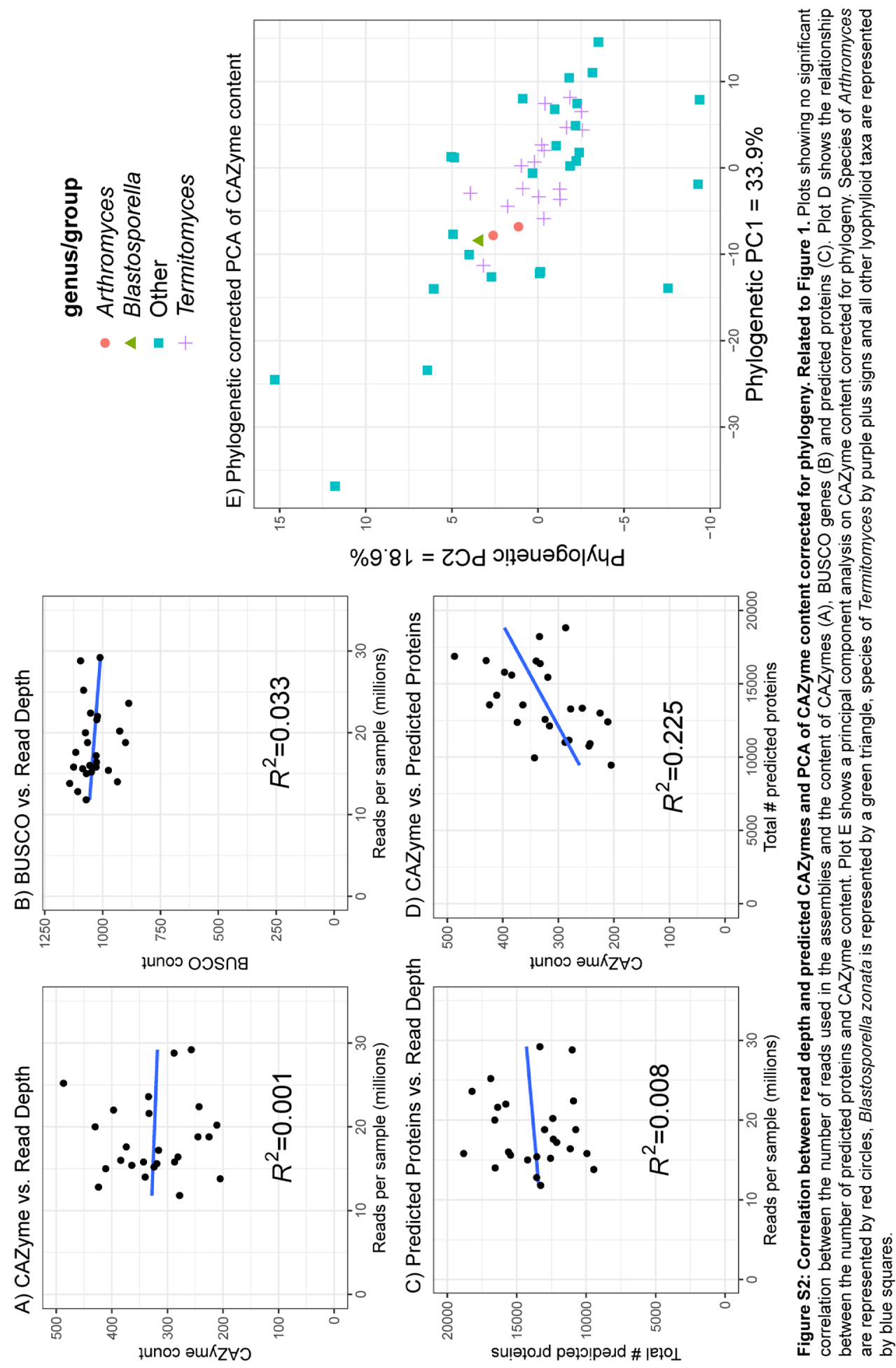




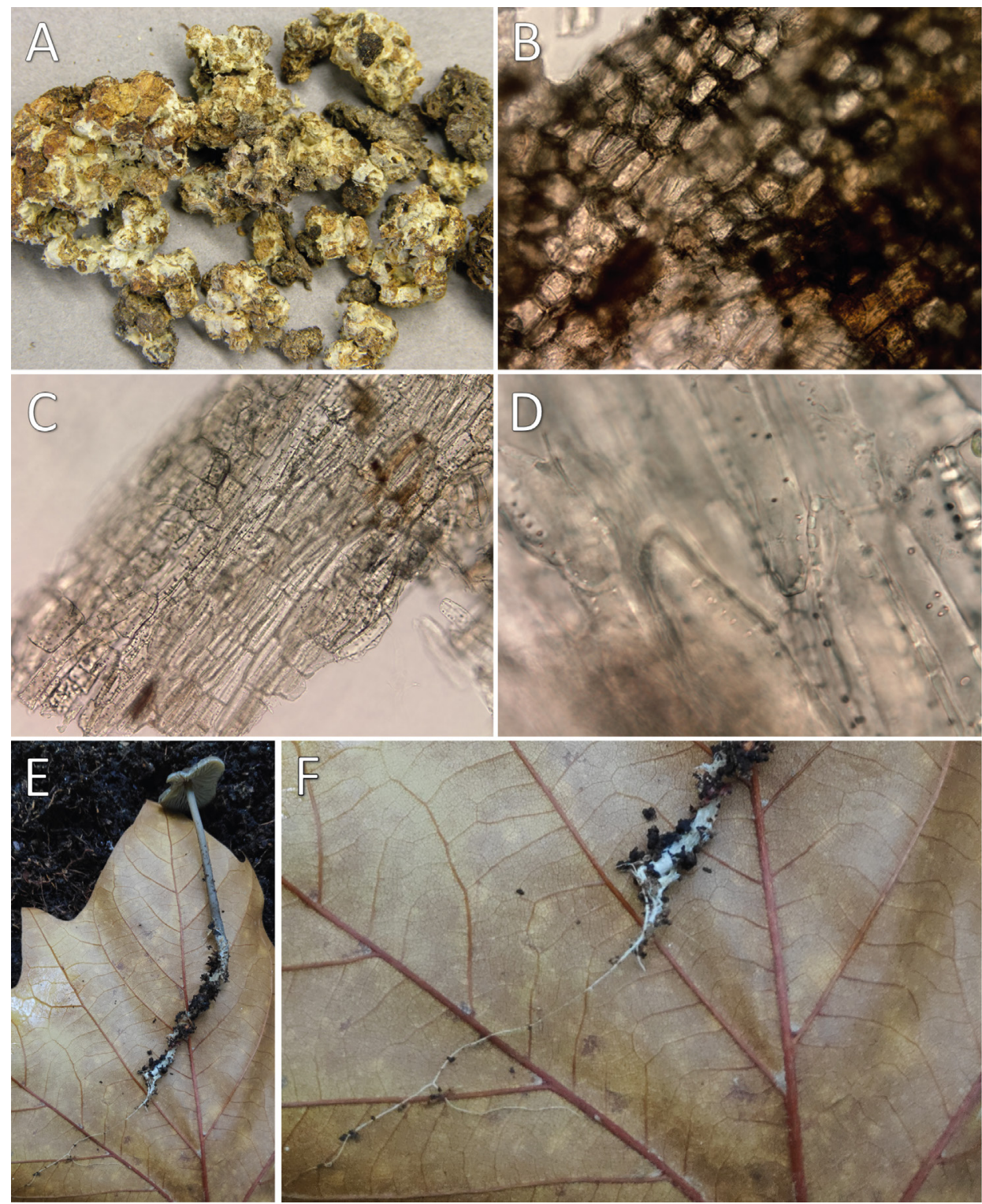

Figure S3 | Insect faecal substrate of Arthromyces matolae and mushroom of Tephrocybe rancida. Related to Figure 2. Macroscopic picture of faecal substrate (A). Microscopic picture showing cork-like structure (B), vessel elements (C) and (bordered) pits in the cell walls (D). These elements are characteristic for plant xylem indicating a woody origin of the substrate. Mushroom of Tephrocybe rancida with tapering pseudorhiza (E). After careful excavation of the pseudorhiza we could not find any attachment to a buried substrate as the pseudorhiza tapered into a thin thread which would eventually break (F). 

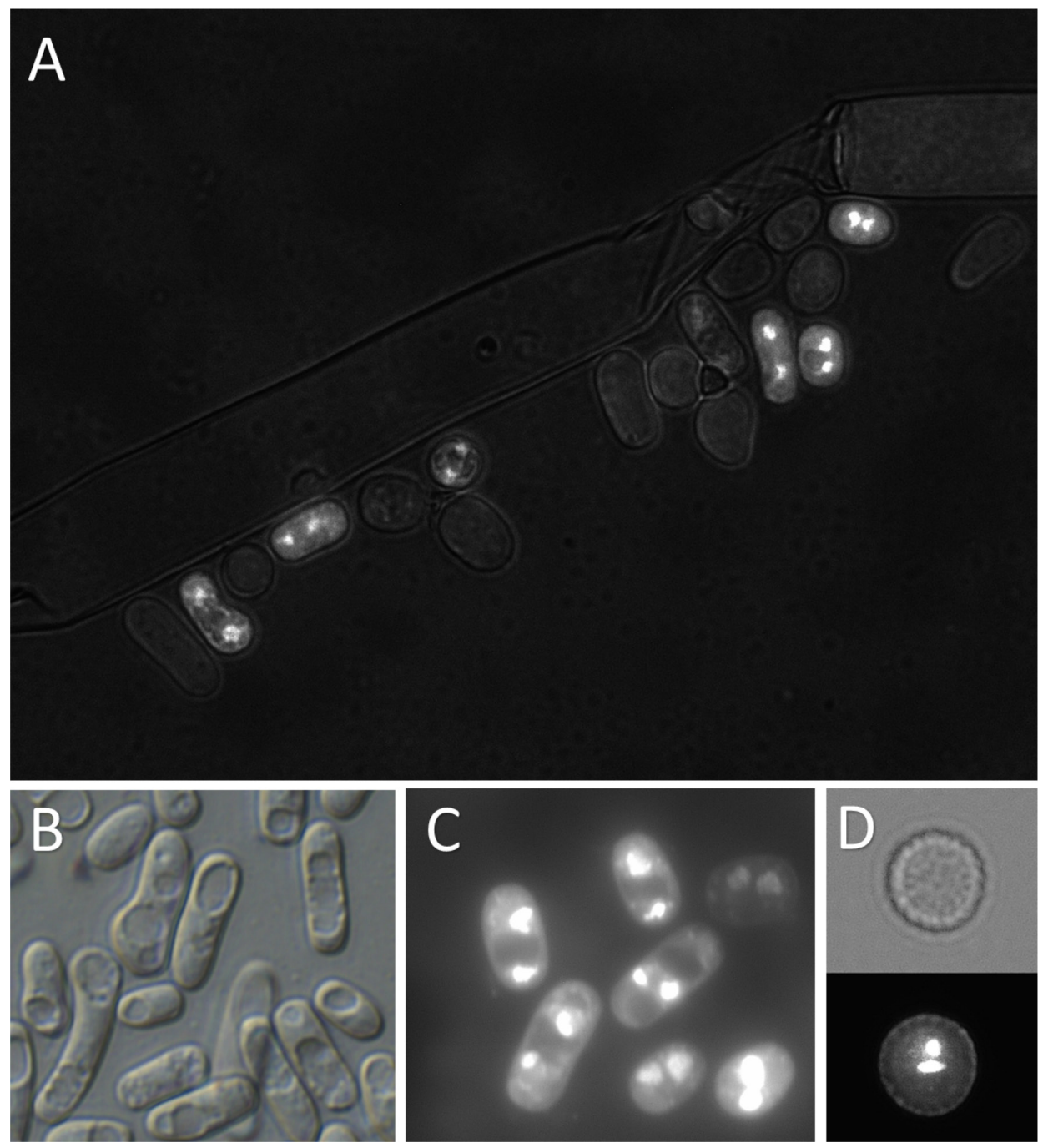

Figure S4 | Production of asexual spores (conidia) in laboratory cultures. Related to Figure 3. Dapi staining of the arthroconidia produced in a culture of Arthromyces matolae (FLAS-F-62734) showing two nuclei per conidium (A). Dikaryotic arthroconidia ( $B$ and $C$ ) and dikaryotic blastoconidia (D) produced in a culture of Blastosporella zonata (Bzo9).

Data S1 Raw CAZyme data showing the number of predicted CAZymes for each class for all species.

Data S2 Sample table with metadata and NCBI Genbank accession numbers for each species.

Available at: https://doi.org/10.1016/j.cub.2021.07.070 



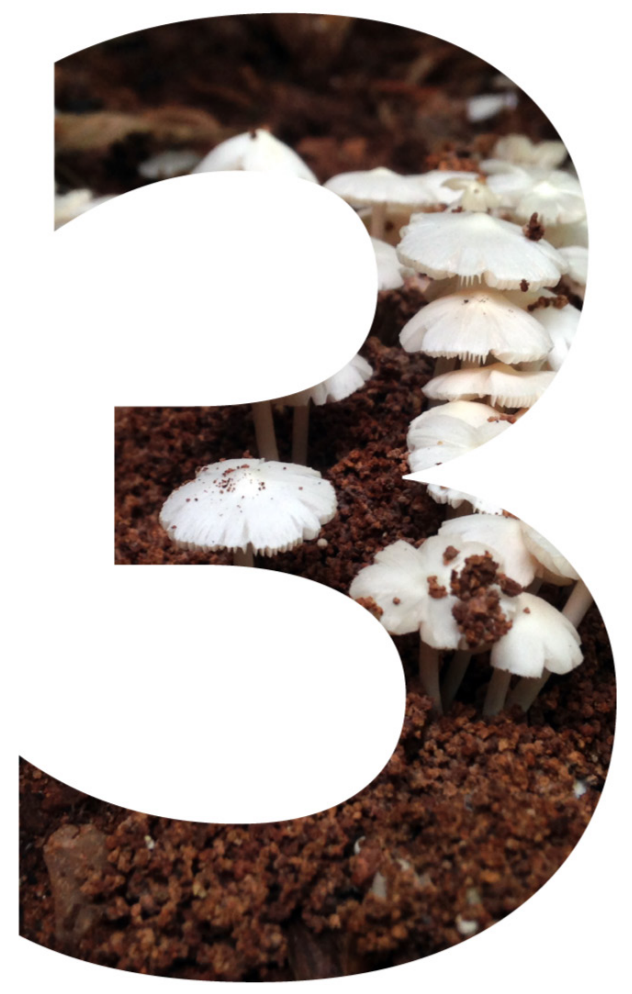

Genetic population structure of the agaric Blastosporella zonata reveals cryptic species and different roles for sexual and asexual spores in dispersal

Lennart J.J. van de Peppel Timothy J. Baroni Duur K. Aanen 


\begin{abstract}
Blastosporella zonata is one of the few basidiomycete fungi that produce asexual spores (conidia) on the mushroom. The role of these conidia in the fungal lifecycle is not known. We tested whether conidia are being utilized in local dispersal by looking for signatures of clonality in 21 samples from three localities separated by about three kilometres in Murillo, Colombia. To identify clonally related individuals, we sequenced three polymorphic markers at two unlinked loci (nuclear rRNA: ITS and LSU, and EF1 $\alpha$ ) for all collections plus three herbarium samples. We identified two sets of clonally related individuals growing closely together in one of the three localities, and only one pair shared between localities. In all three localities we observed multiple nonclonally related dikaryons showing that sexual reproduction is also important. Our results indicate that the conidia on the mushroom are primarily important for local dispersal. Unexpectedly, our results also indicate the existence of two cryptic biological species.
\end{abstract}




\section{Introduction}

The basidiomycete fungus Blastosporella zonata (family: Lyophyllaceae), currently the only species in this genus, is found in the neotropics in the Dominican Republic and Colombia (Baroni et al. 2007). A recent study showed that, within the Lyophyllaceae, B. zonata is part of a termitomycetoid clade which contains the termite-associated fungus Termitomyces (van de Peppel et al. 2021). The same study also showed that $B$. zonata is insect-associated as it grows on faecal pellets, probably of an unidentified Coleopteran larva.

The basidiocarp of $B$. zonata is characterized by its zonate pileus and the production of dark ornamented blastoconidia (Figure 1). In basidiomycetes the formation of asexual spores on the sexual structures is considered rare (Kües et al. 2016); it has been observed only in a small number of species including other members of the Lyophyllaceae, such as the arthroconidia-producing genus Arthromyces (Baroni et al. 2007) and chlamydospore-producing mycoparasites in the genus Asterophora (Buller 1924, Redhead and Seifert 2001). Another arthroconidia-producing agaric which shows resemblance to Arthromyces is Arthrosporella ditopa (Singer 1970), but after examination of the type collection it remains inconclusive whether these two species are closely related (Baroni et al. 2007). Other nonlyophylloid agarics that produce asexual spores on the basidiocarp include the mycoparasites in the genera Squamanita (Bas 1965) and Dendrocollybia (Hughes et al. 2001). Furthermore, the species Pleurotus cystidiosus is known to produce arthroconidia on both the mycelium and the mushroom (Zervakis, Moncalvo, and Vilgalys 2004) and two species of Cystoderma also produce conidia on the mushroom (Saar, Põldmaa, and Kõljalg 2009).

The function of the conidia on the mushroom has been studied in some mycoparasites such as in the genus Asterophora. Two researchers in the 1800's already did experiments in which they infected host mushrooms with chlamydospores (Krombholz 1831, Brefeld 1889) summarized by Buller 1924). They observed that As. lycoperdoides mushrooms emerged on host mushrooms within three weeks after infection. This clearly demonstrates that As. lycoperdoides is able to complete its lifecycle with just the chlamydospores and that these spores can be utilized for host infection. A similar test has not been conducted for species in the genus Squamanita, but the conidia probably serve a similar function in the host infection process (Redhead et al. 1994, Griffith et al. 2019). The utilization of dikaryotic asexual spores instead of monokaryotic sexual spores by these mycoparasites 
makes sense as host mushrooms are ephemeral and decay quickly, therefore their lifecycle is significantly shortened by not having to find a compatible mate.
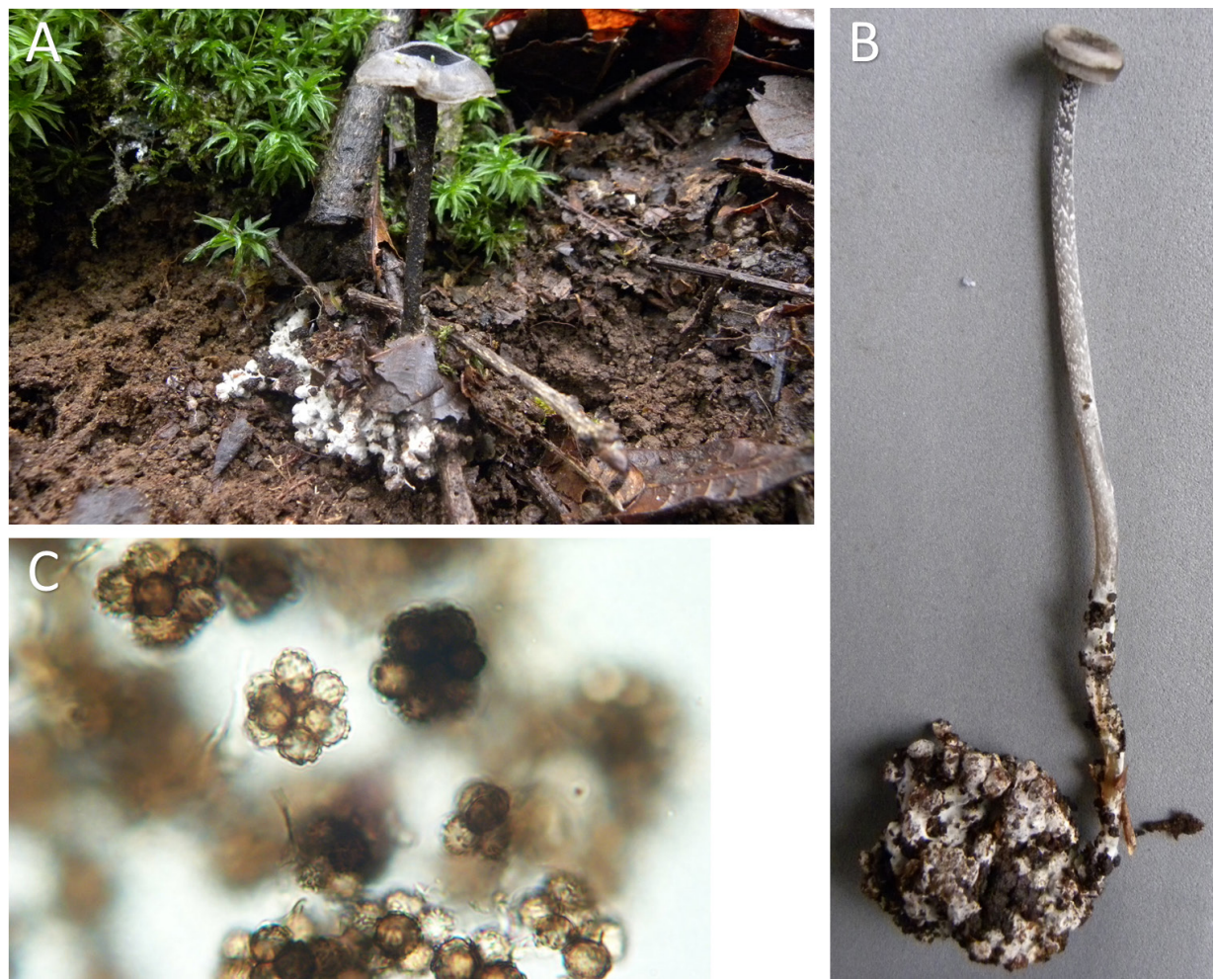
Figure 1 | A: Mushroom of B. zonata (TJB10425). B: Excavated specimen
showing the aggregated faecal pellet substrate (TJB10433). C: Ornamented blastoconidia which cover the pileus (ZT6333).

No studies have been conducted on the role of the conidia in the lifecycle of $B$. zonata, although involvement in substrate colonization, potentially vectored by insects, has been suggested (van de Peppel et al. 2021). B. zonata produces both basidiospores and conidia, and it seems likely that the wind-dispersed basidiospores are used for longrange dispersal, while the non-wind dispersed conidia are used for local dispersal (van de Peppel et al. 2021). In addition the thick-walled conidia may also serve as a survival structure as an insect faecal substrate is most likely only seasonally available. A similar survival function has also been suggested for the conidia of the mycoparasitic genera Asterophora and Squamanita (Redhead et al. 1994).

If $B$. zonata would use the conidia for local dispersal we would expect to see signatures of clonality within and not between 
populations while different populations generally contain different clones that originate from independent colonisations by sexual spores. To test this hypothesis, we collected $B$. zonata mushrooms at three different sites near Murillo, Colombia. To test for genetic variation among our 21 collections of $B$. zonata we obtained DNA sequence data of three highly polymorphic genetic markers.

\section{Material \& Methods}

\section{Sampling of B. zonata}

Blastosporella zonata is a very peculiar basidiomycete fungus, with a very specific ecology growing on isolated substrates of patches of insect faeces. The species is rare, has a patchy distribution and has only been documented from a few countries in South America, growing in cloud forest (Baroni et al. 2007). We collected B. zonata mushrooms at three different sites in a cloud forest close to the village of Murillo, Colombia. Collecting took place between the $31^{\text {st }}$ of October and the $2^{\text {nd }}$ of November 2016. A total of 21 mushrooms were collected. GPS coordinates were recorded for most collections except for four collections (Bzo2, Bzo5, Bzo18 and Bzo19), for which the coordinates were estimated based on the position of nearby collections. Mushrooms were stored in $25 \mathrm{ml}$ tubes and tissue and conidial cultures were made on the same day. Tissue cultures were made by cutting the stipe or pileus of a mushroom and placing a piece of tissue with sterile forceps on a petri dish containing malt yeast extract agar with streptomycin (MYA; per litre demi water: $20 \mathrm{~g}$ malt extract, $2 \mathrm{~g}$ yeast extract, $15 \mathrm{~g}$ agar and $100 \mathrm{ppm}$ streptomycin added after autoclaving). Conidial cultures were made by striking out conidia from the pileus of the mushroom with a sterile needle onto a MYA petri dish. Cultures were first stored at room temperature at the field location and back in the laboratory at $19^{\circ} \mathrm{C}$. Subsequent sub-culturing was done to obtain axenic cultures. Small leftover fragments from mushrooms were stored in $1.5 \mathrm{ml}$ Eppendorf tubes with pure ethanol to serve as backup in case tissue or conidial cultures would not grow. Two herbarium samples collected at the same location and one herbarium sample from the Dominican Republic were also added to the dataset.

\section{Optimal growth temperature test and germination of basidiospores of B. zonata}

To test the optimal growth temperature for $B$. zonata we grew replicate cultures of the same strain (Bzo 9) at six different temperatures $\left(4^{\circ} \mathrm{C}\right.$, $10^{\circ} \mathrm{C}, 15^{\circ} \mathrm{C}, 19^{\circ} \mathrm{C}, 25^{\circ} \mathrm{C}$ and $30^{\circ} \mathrm{C}$ ) on $20 \mathrm{ml}$ of $\mathrm{MA}$ (per litre demi water: 
$8 \mathrm{~g}$ malt extract, $15 \mathrm{~g}$ agar). The inoculum was standardized to a $3 \mathrm{~mm}$ diameter circle by using a sterile corkborer. Cultures were grown for 44 days and scans were made of each culture at day 5, 12, 19, 26 and 44 using an Epson Perfection V600 scanner. The entire experiment was done in triplicate.

The change in total area of a fungal colony over time was used as a measure for growth. As fast growth could go at the cost of producing thin mycelium we recorded white values of the colony as a measure for mycelial density. The area and white value of the fungal colony in each scan were measured using a script in ImageJ (Supplemental file 1).

We attempted to acquire homokaryotic isolates by germinating basidiospores. Basidiospores were collected by attaching the pileus of a mushroom to the lid of a petri dish. The lid was placed on a petri dish with MYA medium and turned after intervals of five seconds, 30 seconds, five minutes and ten minutes. Plates with basidiospores were also incubated at room temperature first and later moved to $19^{\circ} \mathrm{C}$. Individual colonies were quickly subcultured to prevent mating with neighbouring colonies.

\section{DNA isolation, PCR and sequencing}

DNA was extracted from a small piece of cultured mycelium $(0.5 \mathrm{~g})$. In case a tissue or conidial culture was unsuccessful a small piece similar in size of the back-up mushroom stored in ethanol was used instead. All DNA extractions were done using a cetyltrimethylammonium bromide (CTAB) protocol (Nieuwenhuis et al. 2019).

Two partial sequences of the nuclear ribosomal DNA and one fragment of the single-copy nuclear elongation factor 1 alpha (EF1 $\alpha$ ) were amplified using regular polymerase chain reaction (PCR) protocols. The first nuclear ribosomal marker, containing the internal transcribed spacer 1 (ITS1), 5.8S and the internal transcribed spacer 2 (ITS2) was amplified using the fungal specific forward primer ITS1 $f$ and general reverse primer ITS4 (White et al. 1990, Gardes and Bruns 1993). The second nuclear ribosomal marker, a partial sequence of the large ribosomal subunit (LSU, 28S) was amplified using the primers LROR and LR5 (Vilgalys and Hester 1990). A partial sequence of translation elongation factor 1-alpha (EF1 $\alpha$ ) was amplified using the primer pair EF595F and EF1160R (Kauserud and Schumacher 2001). The PCR programs were conducted according to (van de Peppel, Aanen, and Biedermann 2018). 


\section{Chromatograms, alignment, haplotype reconstruction and phylogenetic reconstruction}

The secondary mycelium, regenerated from a mushroom or conidium, contains two genetically different nuclei, which resulted in heterozygous sites in some of the sequences that we generated. We manually checked the chromatograms to identify double peaks using Geneious 10.0.9 (www.geneious.com)(Kearse et al. 2012). Double peaks were assigned with the letter according to the standard International Union of Pure and Applied Chemistry (IUPAC) notation for DNA. Some EF1 $\alpha$ sequences contained a large poly-T repeat of up to ten bases in length in some of the strains. The signal after the poly-T repeat was very distorted so the reverse complement sequence of the reverse primer was used to obtain the remaining part of the sequence. The poly-T region did not affect the outcome of the analyses and was therefore not removed for the final analysis.

Alignments were created for each individual marker. An overview of the sequences and their corresponding Genbank accession numbers can be found in Supplemental table 1. Sequences were aligned using the web server of MAFFT Multiple Sequence Alignment software version 7 using the default settings (Katoh and Standley 2013). After trimming, the alignments for each marker were concatenated using Geneious 10.0.9. After manual inspection in Unipro UGENE variable positions were extracted (Okonechnikov et al. 2012). Haplotypes for each unique genotype were reconstructed using DnaSP 6 (Rozas et al. 2017). Phylogenetic reconstruction for unique haplotypes was conducted using the webserver of IQ-tree with defaults settings and 1000 ultrafast bootstraps (Trifinopoulos et al. 2016).

\section{Results}

\section{Optimal growth temperature test and germination of basidiospores of B.zonata}

The results of our growth experiment show a clear pattern, both total area and white value were highest at $19^{\circ} \mathrm{C}$ (Supplemental figure 1). At $4^{\circ} \mathrm{C}, 10^{\circ} \mathrm{C}$ and $15^{\circ} \mathrm{C}$ the culture was still able to grow but the total area was smaller, and white values were very similar suggesting similar mycelial densities at these temperatures. At $25^{\circ} \mathrm{C}$ degrees colony growth was much lower and also white values were lower compared to the other treatments. At $30^{\circ} \mathrm{C}$ none of the replicates showed any radial growth.

Our attempts at germinating the blastoconidia of $B$. zonata were highly successful with very low rates of bacterial or fungal infection. 
Basidiospores had a much lower germination rate and took up to several weeks to germinate. We obtained seven homokaryotic cultures from a single parent (Figure 2).

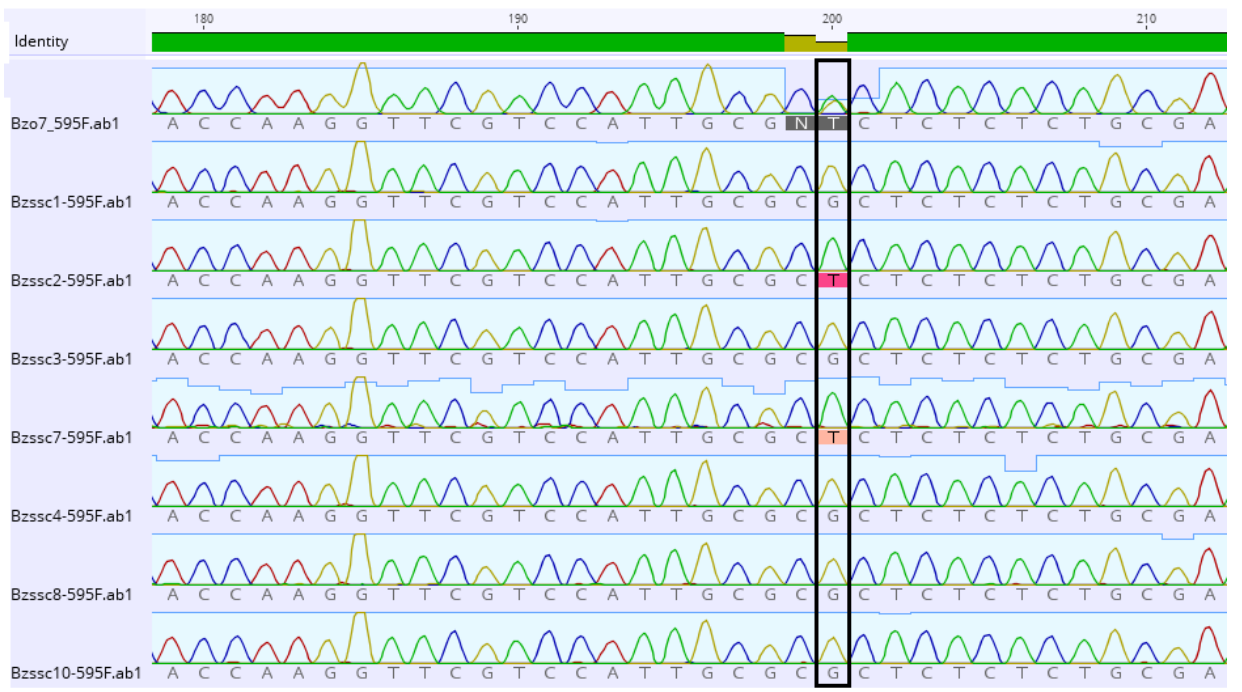

Figure 2 Chromatograms of partial EF1 $\alpha$ sequences of B. zonata (Bzo7) showing the dikaryotic (G/T) parent (top sequence) and the homokaryotic offspring (either a G or T).

\section{Population study}

We found that the genetic resolution was lowest for the nuclear ribosomal marker (seven variable positions; four in ITS, three in LSU), based on the concatenated alignments of ITS and LSU we could recover five different genotypes. The EF1 $\alpha$ marker offered a much higher genetic resolution (44 variable positions) and yielded a total of 14 different genotypes. Concatenation of the ribosomal marker and EF1 $\alpha$ alignments did not yield a higher resolution than the 14 genotypes that were already recovered using just the EF1 $\alpha$ marker.

Haplotype reconstruction resulted in 19 different haplotypes from the 14 different genotypes (Figure 3A). Phylogenetic reconstruction of the reconstructed haplotypes revealed two distinct clades (Figure 3B). Individual haplotypes of a genotype always belonged to one of those two clades. Assuming a single, randomly mating population, the probability that the eight genotypes from clade 1, and the six genotypes from clade 2, all contain haplotypes of their respective clades is:

$$
p=\left(\frac{16}{28}\right)^{8} *\left(\frac{12}{28}\right)^{6}=7.0 * 10^{-5}
$$


Therefore, we conclude that the two clades represent two cryptic biological species within $B$. zonata.

\begin{tabular}{|l|c|c|}
\hline Collection & Genotype & Site \\
\hline Bzo 1 & $1-1$ & 1 \\
\hline Bzo 2 & $2-2$ & 1 \\
\hline Bzo 3 & $1-3$ & 1 \\
\hline Bzo 4 & $4-5$ & 2 \\
\hline Bzo 5 & $6-7$ & 2 \\
\hline Bzo 6 & 6-8 & 2 \\
\hline Bzo 7 & $9-10$ & 2 \\
\hline Bzo 8 & $4-11$ & 2 \\
\hline Bzo 9 & $7-12$ & 3 \\
\hline Bzo 10 & $2-13$ & 3 \\
\hline Bzo 11 & $2-13$ & 3 \\
\hline Bzo 12 & $2-13$ & 3 \\
\hline Bzo 13 & $2-13$ & 3 \\
\hline Bzo 14 & $2-13$ & 3 \\
\hline Bzo 15 & $2-13$ & 3 \\
\hline Bzo 16 & $13-14$ & 3 \\
\hline Bzo 17 & $13-14$ & 3 \\
\hline Bzo 18 & $2-13$ & 3 \\
\hline Bzo 19 & $2-13$ & 3 \\
\hline Bzo 20 & $2-13$ & 3 \\
\hline Bzo 21 & $15-16$ & 3 \\
\hline TJB10425 & $15-16$ & 1 \\
\hline TJB10431 & $17-18$ & 2 \\
\hline TJB8371 & $19-19$ & DR \\
\hline
\end{tabular}
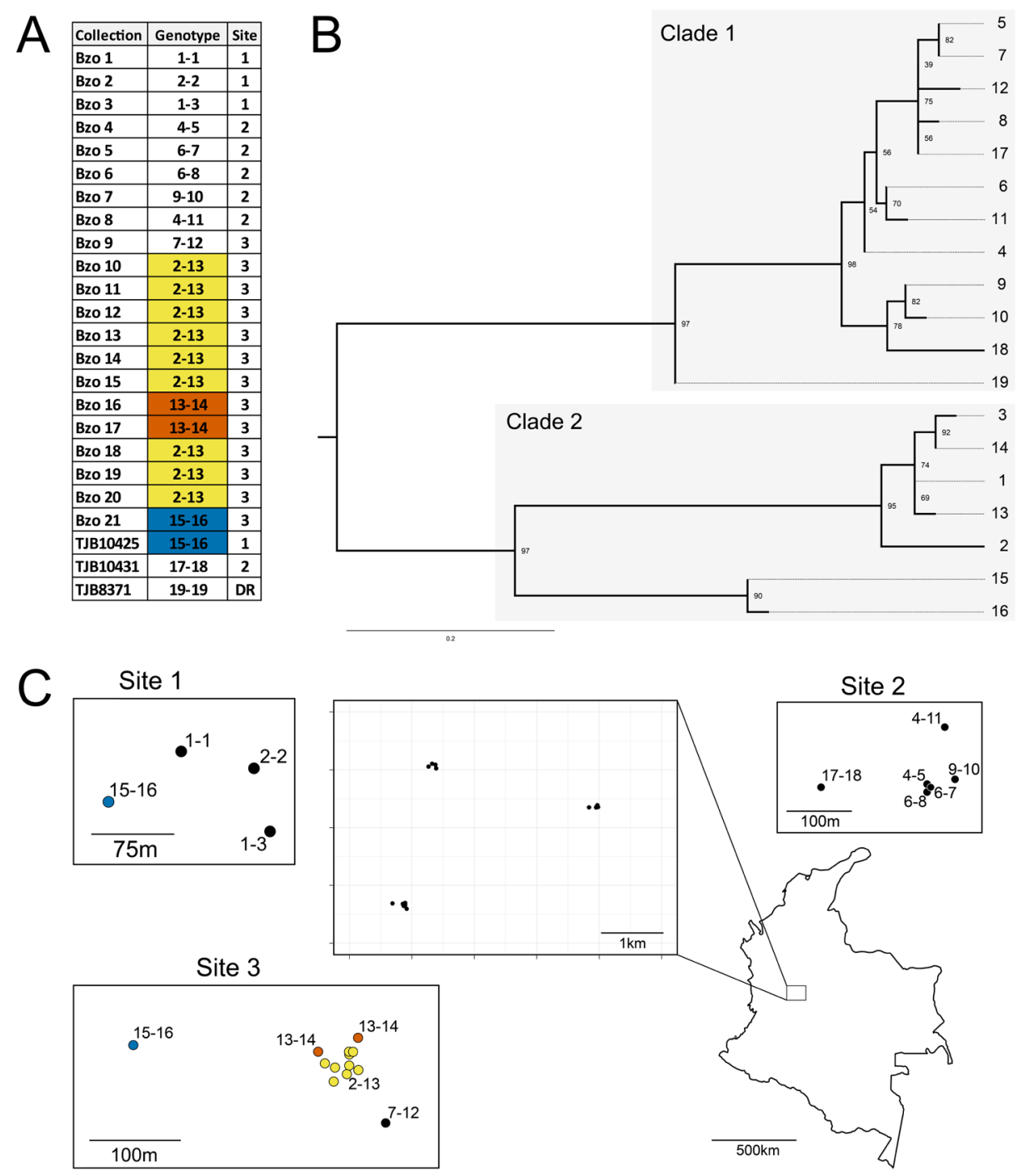

Figure 3 | A: Collections of $B$. zonata with their corresponding genotypes (predicted haplotype 1 - predicted haplotype 2) and collection site (Site 1,2,3 or Dominican Republic (DR)). Collections with identical genotypes are highlighted in blue, red or yellow. B: Maximum-likelihood tree of predicted haplotypes with midpoint rooting, numbers at the nodes indicate ultrafast bootstraps. C: Map of the collections of $B$. zonata at the three different sites around Murillo, Colombia. Presumed clones are highlighted using the same colours used in panel A. 
In total we found three genotypes that occurred more than once, two twice (15-16 and 13-14), and one nine times (2-13). We found three unique genotypes at site 1 and one genotype (15-16) at this site also occurred at site 3 (Figure 3C). All genotypes at site 3 belonged to clade 2. Site 2 harboured six unique genotypes all belonging to clade 1 (Figure 3C). Site 3 contained one individual of clade 1, this genotype was shared with site 1 (15-16), the larger group of nine individuals from clade 2 and the smaller group of two individuals belong to clade 2 .

Focusing on the clade where we found identical genotypes (clade 2), we calculated expected genotype frequencies under random mating and compared the expected frequencies with the observed frequencies. This showed a statistically highly significant difference $(x 2$ $=61.55 ; 36$ degrees of freedom; $p=0.001$ ), demonstrating that this population deviates from free recombination. This is most likely due to clonality of some of the samples, particularly since those are also mostly located within one site. Theoretically, an alternative explanation would be inbreeding (although this would lead to a higher frequency of homozygous samples, which we did not find). Therefore, we also tested for deviations within the site where we found the identical genotypes (site 3$)$. This analysis showed an even higher $p$ value $(x 2=36.00 ; 16$ degrees of freedom; $p=0.0009)$. We therefore conclude that these samples are clonality related.

\section{Discussion}

Our experiment on the optimal growth temperature of $B$. zonata shows that this species grows best at $19^{\circ} \mathrm{C}$, while it is also able to grow at lower temperatures as low $4^{\circ} \mathrm{C}$. At $25^{\circ} \mathrm{C}$ growth speed is severely reduced while at $30^{\circ} \mathrm{C}$ cultures were unable to grow. These findings show that $B$. zonata is well-adapted to grow at the colder temperatures in cloud forests (Jarvis and Mulligan 2011). This is sharp contrast with the termite-associated genus Termitomyces of which a number of species have an optimal growth temperature around $29^{\circ} \mathrm{C}$ (Thomas 1981).

At the two sites with the fewest collections, sites 1 and 2, we found a similar pattern with mostly unique genotypes, and the samples at each site belonging to one of the two clades, and even mushrooms growing close to each other differ genetically (Figure 3C). At site 3 the majority of individuals belonged to clade 2 with only a single collection from clade 1 (Figure 3C). At this site we found two groups with identical sequences for all three markers; genotype 13-14 (two individuals) and genotype 2-13 (nine individuals), and one individual had an identical genotype (15-16) to a collection at site 1. Given the total genetic 
diversity we found using the two genetic markers, the identical sequences for all markers for two groups of individuals point towards clonality. Our phylogenetic analysis on the predicted haplotypes for each genotype revealed that both predicted haplotypes of a single genotype always belonged to the same clade which indicates reproductive isolation between the two clades. From this, we conclude that clade 1 and 2 represent two cryptic species within $B$. zonata. We do not know whether there is any ecological differentiation between the two, for example due to differences in host-specificity.

All of the mushrooms we collected were attached to aggregated faecal pellets, which shows the dependence of $B$. zonata on this substrate to complete its life cycle. As the substrate is heterogeneous and may only be seasonally available, depending on the life cycle of the insect, we expect that the blastoconidia from B. zonata may enhance successful substrate colonization. Dispersal via conidia is very convenient for a fungus which grows on a rare and seasonal substrate because only a single conidium is needed for colonization and completion of the lifecycle via the production of mushrooms. Colonization of such temporal substrates via sexual spores is less certain in heterothallic species, as it always requires two sexually compatible basidiospores to form mushrooms. The clonality we find at site 3 suggests that blastoconidia on the mushroom are particularly important for local dispersal. However, long-range dispersal might occasionally happen as we found two genetically identical individuals, which likely are clonally related, at sites 1 and 3 , separated by about three kilometres. We did not observe any substantial mycelial growth extending outwards from the faecal substrate into the soil, and substrates were physically separated by soil, so colonization via mycelial outgrowth is unlikely. The blastoconidia from $B$. zonata are thick-walled and pigmented, which indicates that they are adapted for long-term survival (Clémençon 1997, Kües et al. 2016). The conidia may remain dormant in the soil until new faecal pellets are produced by the host insect. The thick-walled chlamydospores of Squamanita and Asterophora may have a similar role as the spores remain dormant in the soil until new host mushrooms appear (Redhead et al. 1994). It has been assumed that Asterophora mainly uses the chlamydospores to complete its lifecycle (Buller 1924). This is supported by the observation that basidiospores are often completely absent in mature mushrooms of these fungi (de Bary 1859, Buller 1924, Thompson 1936), but occasional mushrooms with basidiospores also have been reported (Brefeld 1889, Ingold 1940). Studies on three species of Squamanita found no polymorphisms in the ITS1 sequence within each species, 
indicating predominantly asexual reproduction (Mondiet, Dubois, and Selosse 2007, Griffith et al. 2019). In our study on B. zonata we observe ample genetic diversity, especially at sites 1 and 2, indicating that basidiospores play an important role in shaping the genetic population structure. We also did not observe malformed or absent lamellae in mature mushrooms of $B$. zonata, which is sometimes the case for mushrooms of Asterophora (Redhead and Seifert 2001). In one case (Bzo 7) we managed to germinate basidiospores of $B$. zonata, confirming that they are viable. In the case of Asterophora, both wind dispersal (Buller 1924) and arthropod dispersal have been suggested (Redhead et al. 1994). Since B. zonata grows on insect pellets, a potential insect dispersal agent of the blastoconidia seems likely and deserves further investigation.

\section{Acknowledgements}

We want to thank Ana Esperanza Franco-Molano for the organization of the field trip to Murillo and Martha Cárdenas for assistance in the lab. We thank Alex Grum-Grzhimaylo and the Colombian students for helping us spot and collect mushrooms. We want to thank Ben Auxier for his assistance with creating Figure 3. We want to thank Natascha Oosterwijk for conducting the growth temperature experiment. 


\section{Supplementary materials}

Supplemental table 1 | Overview of the samples collected and used during this study with their corresponding Genbank accession numbers, collection site, genotype, haplotypes, collection date and location.

\begin{tabular}{|c|c|c|c|c|c|c|c|c|c|}
\hline Collection & ITS & LSU & EF1 $\alpha$ & Genotype & Site & hap1 & hap2 & Collected & Coordinates \\
\hline Bzo 1 & MZ566499 & MZ566495 & MZ574147 & 1-1 & 1 & 1 & 1 & $31 / 10 / 2016$ & N $04^{\circ} 52^{\prime} 51.83^{\prime \prime}$ W $075^{\circ} 10^{\prime} 00.44^{\prime \prime}$ \\
\hline Bzo 2 & MZ566499 & MZ566495 & MZ574148 & $2-2$ & 1 & 2 & 2 & $31 / 10 / 2016$ & Estimated \\
\hline Bzo 3 & MZ566501 & MZ566495 & MZ574149 & -3 & 1 & 1 & 3 & $31 / 10 / 2016$ & 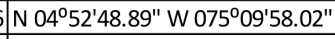 \\
\hline Bzo 4 & MZ566498 & MZ566494 & MZ574150 & $4-5$ & 2 & 4 & 5 & $01 / 11 / 2016$ & N $04^{\circ} 52^{\prime} 24.69^{\prime \prime}$ W $075^{\circ} 08^{\prime} 25.78^{\prime \prime}$ \\
\hline Bzo 5 & MZ566498 & MZ566494 & MZ574151 & 6 & 2 & 6 & 7 & $01 / 11 / 2016$ & Estimated \\
\hline Bzo 6 & MZ566498 & MZ566494 & MZ574152 & 6-8 & 2 & 6 & 8 & $01 / 11 / 2016$ & N $04^{\circ} 52^{\prime} 24.50^{\prime \prime}$ W $075^{\circ} 08^{\prime} 25.84^{\prime \prime}$ \\
\hline Bzo 7 & MZ566498 & MZ566494 & MZ574153 & $9-10$ & 2 & 9 & 10 & $01 / 11 / 2016$ & $\mathrm{~N} 04^{\circ} 52^{\prime} 24.82^{\prime \prime}$ W $075^{\circ} 08^{\prime} 24.69^{\prime \prime}$ \\
\hline Bzo 8 & MZ566498 & MZ566494 & MZ574154 & $11-4$ & 2 & 11 & 4 & $01 / 11 / 2016$ & 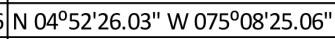 \\
\hline Bzo 9 & MZ566498 & MZ566494 & MZ574155 & $7-12$ & 3 & 7 & 12 & $02 / 11 / 2016$ & N $04^{\circ} 51^{\prime} 21.41^{\prime \prime}$ W $075^{\circ} 10^{\prime} 15$ \\
\hline Bzo 10 & MZ566499 & MZ566495 & MZ574156 & $13-2$ & 3 & 13 & 2 & $02 / 11 / 2016$ & 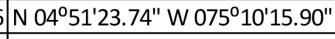 \\
\hline Bzo 11 & MZ566499 & MZ566495 & MZ574156 & 13-2 & 3 & 13 & 2 & $02 / 11 / 2016$ & N $04^{\circ} 51^{\prime} 23.91^{\prime \prime}$ W $075^{\circ} 10^{\prime} 16.22^{\prime \prime}$ \\
\hline Bzo 12 & MZ566499 & MZ566495 & MZ574156 & & 3 & 13 & 2 & & $\mathrm{~N} 04^{\circ} 51^{\prime} 24.50^{\prime \prime} \mathrm{W} 075^{\circ} 10^{\prime} 16.09^{\prime \prime}$ \\
\hline Bzo 13 & MZ566499 & MZ566495 & MZ574156 & 13-2 & 3 & 13 & 2 & $02 / 11 / 2016$ & N $04^{\circ} 51^{\prime} 24.41^{\prime \prime}$ W $075^{\circ} 10^{\prime} 16.22^{\prime \prime}$ \\
\hline Bzo 14 & MZ566499 & MZ566495 & MZ574156 & 13-2 & 3 & 13 & 2 & $02 / 11 / 2016$ & N $04^{\circ} 51^{\prime} 24.50^{\prime \prime}$ W $075^{\circ} 10^{\prime} 16.23^{\prime \prime}$ \\
\hline Bzo 15 & MZ566499 & MZ566495 & MZ574156 & & 3 & 13 & 2 & $02 / 11 / 2016$ & N $04^{\circ} 51^{\prime} 23.96^{\prime \prime}$ W $075^{\circ} 10^{\prime} 16.98^{\prime \prime}$ \\
\hline Bzo 16 & MZ566499 & MZ566495 & MZ574157 & 13-14 & 3 & 13 & 14 & $02 / 11 / 2016$ & N $04^{\circ} 51^{\prime} 25.11^{\prime \prime}$ W $075^{\circ} 10^{\prime} 15.89^{\prime \prime}$ \\
\hline Bzo 17 & MZ566499 & MZ566495 & MZ574157 & 13-14 & 3 & 13 & 14 & $02 / 11 / 2016$ & N $04^{\circ} 51^{\prime} 24.49^{\prime \prime}$ W $075^{\circ} 10^{\prime} 17.23^{\prime \prime}$ \\
\hline Bzo 18 & MZ566499 & MZ566495 & MZ574156 & $13-2$ & 3 & 13 & 2 & $02 / 11 / 2016$ & Estimated \\
\hline Bzo 19 & MZ566499 & MZ566495 & MZ574156 & 13-2 & 3 & 13 & 2 & $02 / 11 / 2016$ & Estimated \\
\hline Bzo 20 & MZ566499 & MZ566495 & MZ574156 & $13-2$ & 3 & 13 & 2 & $02 / 11 / 2016$ & N 04 ${ }^{\circ} 51^{\prime} 23.21^{\prime \prime} \mathrm{W} 075^{\circ} 10^{\prime} 16.73^{\prime \prime}$ \\
\hline Bzo 21 & MZ566500 & MZ566496 & MZ574158 & $15-16$ & 3 & 15 & 16 & $02 / 11 / 2016$ & N $04^{\circ} 51^{\prime} 24.78^{\prime \prime}$ W $075^{\circ} 10^{\prime} 23.15^{\prime \prime}$ \\
\hline TJB10425 & MZ566500 & MZ566496 & MZ574158 & 15-16 & 1 & 15 & 16 & & N $04^{\circ} 52^{\prime} 50.00^{\prime \prime} \mathrm{W} 075^{\circ} 10^{\prime} 02.40^{\prime \prime}$ \\
\hline TJB10431 & MZ566498 & MZ566494 & MZ574159 & 17-18 & 2 & 17 & 18 & & N $04^{\circ} 52^{\prime} 24.60^{\prime \prime}$ W $075^{\circ} 08^{\prime} 29.90^{\prime \prime}$ \\
\hline TJB8371 & EU708340 & EU708337 & MZ574160 & 19-19 & DR & 19 & 19 & & N 1902'33.00" W $070^{\circ} 31^{\prime} 03.00^{\prime \prime}$ \\
\hline
\end{tabular}




\section{B. zonata - area}

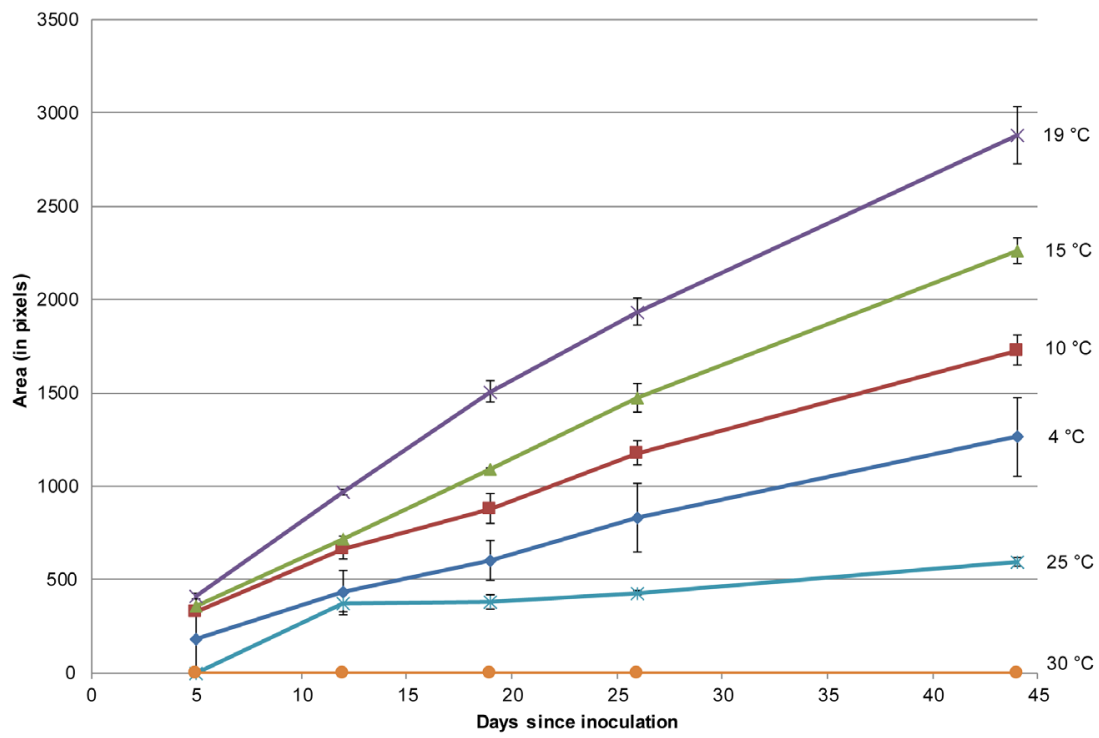

B. zonata - white value

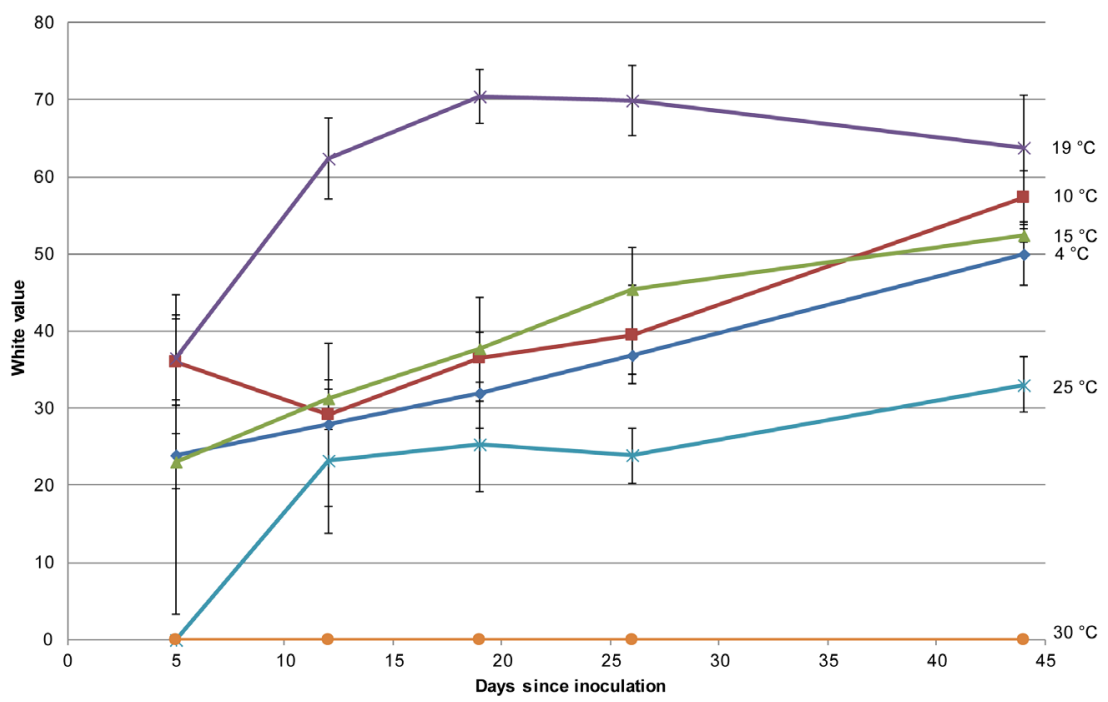

Supplemental figure 1 | Increase of fungal colony area (top) and white value (bottom) over time. Error bars at each data point represent standard deviation. Temperature treatments are given at the far right next to each graph. Both total area and white value were highest after 44 days for cultures grown at $19^{\circ} \mathrm{C}$. None of the cultures kept at $30^{\circ} \mathrm{C}$ showed any growth. 


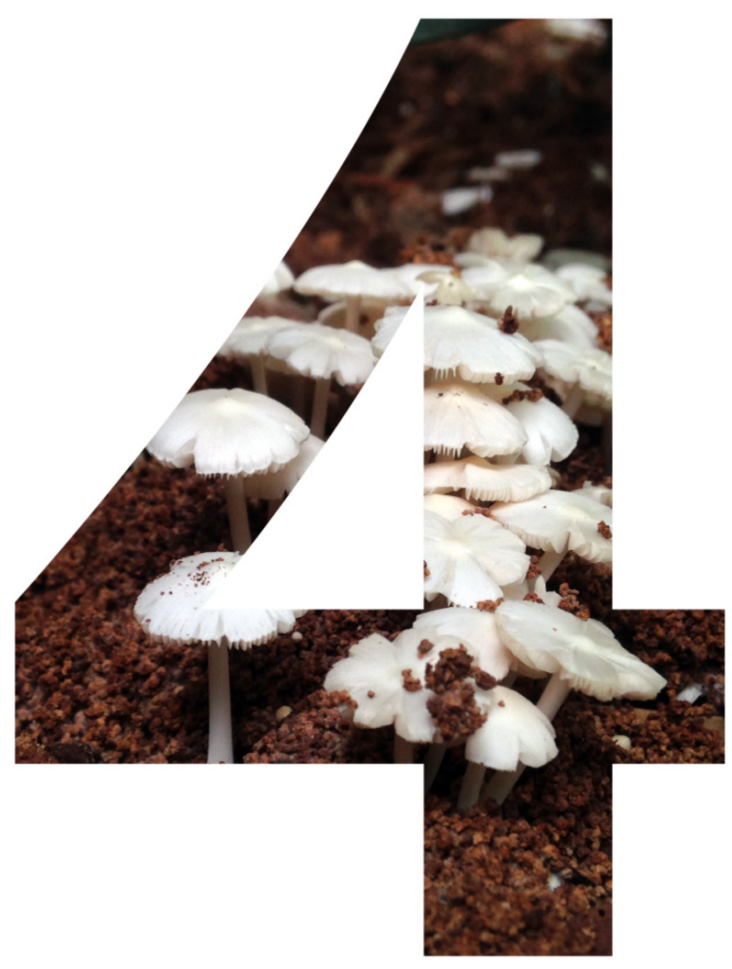

Four new genera and six new species of lyophylloid agarics (Agaricales, Basidiomycota) from three different continents Lennart J.J. van de Peppel, M. Catherine Aime Thomas Læssøe, Ole S. Pedersen Victor R.M. Coimbra, Thomas W. Kuyper Duur K. Aanen, Timothy J. Baroni 


\section{Abstract}

Four new genera encompassing four new species are described in Lyophyllaceae (Agaricales). Australocybe is a novel monotypic genus from Australia. Phaeotephrocybe is a novel monotypic genus from Belize. Nigrocarnea is novel monotypic genus from Laos containing the novel conidia-producing species $N$. radicata. The novel genus Praearthromyces contains two Asian taxa, the conidia-producing Pr. furcatifolius known from Malaysia and Singapore, and Pr. griseus from Thailand, which lacks conidial production. In addition, we describe Arthromyces glabriceps, which is the first species in the genus Arthromyces, which lacks conidial production on the basidiome. Alternative options for a classification of these lyophylloid taxa are discussed and on the basis of morphological, ecological and biogeographical considerations rejected.

* The species names in this chapter will be validated at a later stage in accordance with the International Code of Nomenclature for Algae, Fungi and Plants. 


\section{Introduction}

The genus Tephrocybe as introduced by Donk (1962) as a segregate from the genus Lyophyllum P. Karst., contains a number of small collybioid mushrooms, often with a hygrophanous pileus and grey colours (Donk 1962). Various molecular studies have shown the genus to be paraphyletic, which is consistent with the lack of diagnostic features (Hofstetter et al. 2002, Hofstetter et al. 2014, Bellanger et al. 2015). A number of Tephrocybe species have been transferred to new genera such as Myochromella, Sagaranella and Sphagnurus (Hofstetter et al. 2014). This introduction of new genera made the genus Tephrocybe monotypic with $T$. rancida, the type species. A previous study showed that $T$. rancida is part of a termitomycetoid clade which, next to the termite-associated genus Termitomyces, also contains the insect-faecal associated genera Arthromyces and Blastosporella (van de Peppel et al. 2021). The study by van de Peppel et al. also showed that five different species, which were provisionally identified as Tephrocybe sp. (s.l.), were also part of this termitomycetoid clade. In this study we examined these five unidentified species and an additional specimen of an unidentified species of the genus Arthromyces.

The specimen from Australia (Tephrocybe sp. 1) is macroscopically similar to $T$. rancida but phylogenetically occupies an isolated branch in the termitomycetoid clade. Because of the strong phylogenetic evidence and to conserve monophyletic genera, we propose to place this species in the monotypic genus Australocybe. The sole member of this genus, $A$. olivacea, is described below.

The study by van de Peppel et al. 2021 recovered a subclade within the termitomycetoid clade containing $T$. rancida, $B$. zonata and Tephrocybe sp. 2 and 3. Fieldwork in Laos in connection with the production of the first booklet on edible, poisonous and medicinal fungi from this country led to the discovery of Tephrocybe sp. 2, a species with internal conidia in the pileus context (Læssøe, Pedersen, and Sysouphanthong 2019). Tephrocybe sp. 3 is a species from Belize, similar to $T$. rancida, which also lacks the production of conidia on the basidiome. Due to the lack of shared characters among these two species and the genus Blastosporella, we propose to erect the two monotypic genera Phaeotephrocybe and Nigrocarnea. Tephrocybe sp. 2 is described below as N. radicata and Tephrocybe sp. 3 is described below as $P$. leucophylla.

Baroni et al. (2007) reported a close morphological similarity of the palaeotropical / Asian Tricholoma furcatifolium to the neotropical / American genus Arthromyces. Examination of Tephrocybe sp. 5 from the study by van de Peppel et al. 2021 showed a match of this specimen 
with the description of $T$. furcatifolium. This species together with Tephrocybe sp. 4 make up a small clade sister to the ArthromycesTermitomyces clade, and these two species thus are not related to Tricholoma. In addition, we examined the type collection of $T$. furcatifolium. A DNA sequence generated from one of the paratypes of T. furcatifolium had a perfect match to Tephrocybe sp. 5 and therefore confirms this species belongs to the Lyophyllaceae. As both this species and Tephrocybe sp. 4 do not nest phylogenetically with any of the described genera within the termitomycetoid clade we propose to transfer T. furcatifolium to the new genus Praearthromyces and describe Tephrocybe sp. 4 as Pr. griseus below.

The genus Arthromyces currently encompasses two species, $A$. matolae known from Belize and A. claviformis, originally reported from Puerto Rico and the Dominican Republic (Baroni et al. 2007). Recently, A. claviformis has also been observed in Colombia near Cali (mushroomobserver.org, observations: 275090, 348275, 367362) and Cogua (observation 363150). A recent soil metabarcoding study identified OTU's as Arthromyces sp. from the Andean Yungas forest of Argentina (Eduardo et al. 2018). This indicates that the genus Arthromyces has a distribution range spanning the entire continent of South America and extending to the Caribbean islands and Central America. Furthermore, it has been found further north as $A$. matolae has been collected from Gainesville, Florida (van de Peppel et al. 2021). Although the production of dark arthroconidia on both the stipe and pileus of the basidiome is one of the key features of Arthromyces we examined a specimen, identified as Arthromyces sp., which lacks conidia on the basidiome. Our molecular phylogenetic analysis shows that this species nests within the genus Arthromyces and is sister to $A$. matolae. This species is described as $A$. glabriceps below.

\section{Material and methods}

\section{Morphological analysis}

Colour codes mentioned in the species descriptions refer to Kornerup $\&$ Wanscher (1978) (Kornerup and Wanscher 1978).

\section{DNA extraction and PCR}

For DNA extraction, small fragments of dried fungarium specimens were used. DNA was extracted following a modified cetyltrimethylammonium bromide (CTAB) protocol (Nieuwenhuis et al. 2019). Three markers were amplified using conventional polymerase chain reactions (PCRs); the nuclear ribosomal region containing the 
internal transcribed spacer 1 (ITS1), 5.8S and the internal transcribed spacer 2 (ITS2), a partial sequence of the nuclear ribosomal large subunit (LSU, 28S) and the sequence encoding for elongation factor 1 alpha $(E F 1 \alpha)$. The following primer pairs were used: the fungal specific forward primer ITS1f and the universal reverse primer ITS4 for the ITS marker (White et al. 1990, Gardes and Bruns 1993), LROR and LR5 for the LSU (Vilgalys and Hester 1990) and EF595F and EF1160R for EF1 $\alpha$ (Kauserud and Schumacher 2001). After initial amplification of the holotype of T. furcatifolium failed, a PCR was conducted targeting ITS1 and ITS2 separately using the primer pairs ITS1f/ITS2 and ITS3/ITS4 (White et al. 1990, Gardes and Bruns 1993).

All PCR programs were conducted according to (van de Peppel, Aanen, and Biedermann 2018).

Sequencing was performed by Eurofins genomics (Ebersberg, Germany) using both the forward and reverse primer. For LSU only the forward primer (LROR) was used as the forward read was of sufficient quality for subsequent analysis. Inspection of the chromatograms and assembly of the forward and reverse sequences was done using the CLC Genomics Workbench 8.

\section{Phylogenetic analysis}

As a phylogenomic study was conducted on the termitomycetoid clade (van de Peppel et al. 2021), we limited our phylogenetic reconstruction on specimens of the genus Arthromyces using Termitomyces as an outgroup. Sequences for each individual marker (ITS, LSU and EF1 $\alpha$ ) were aligned using MAFFT v7.409 online alignment software, using default settings (Katoh, Rozewicki, and Yamada 2017). The three resulting alignments were trimmed and concatenated in Geneious 10.0.9 (www.geneious.com)(Kearse et al. 2012). A maximum-likelihood tree was reconstructed using the webserver of $1 \mathrm{Q}$-tree with default settings and 1000 ultrafast bootstraps (Trifinopoulos et al. 2016).

\section{Results}

\section{Phylogenetic analysis}

The maximum-likelihood from the analysis by van de Peppel et al. 2021 is fully resolved (Figure 1A). The phylogenetic reconstruction we conducted on specimens of the genus Arthromyces shows that $A$. glabriceps is sister to a clade containing $A$. matolae and $A$. claviformis (Figure 1B). 


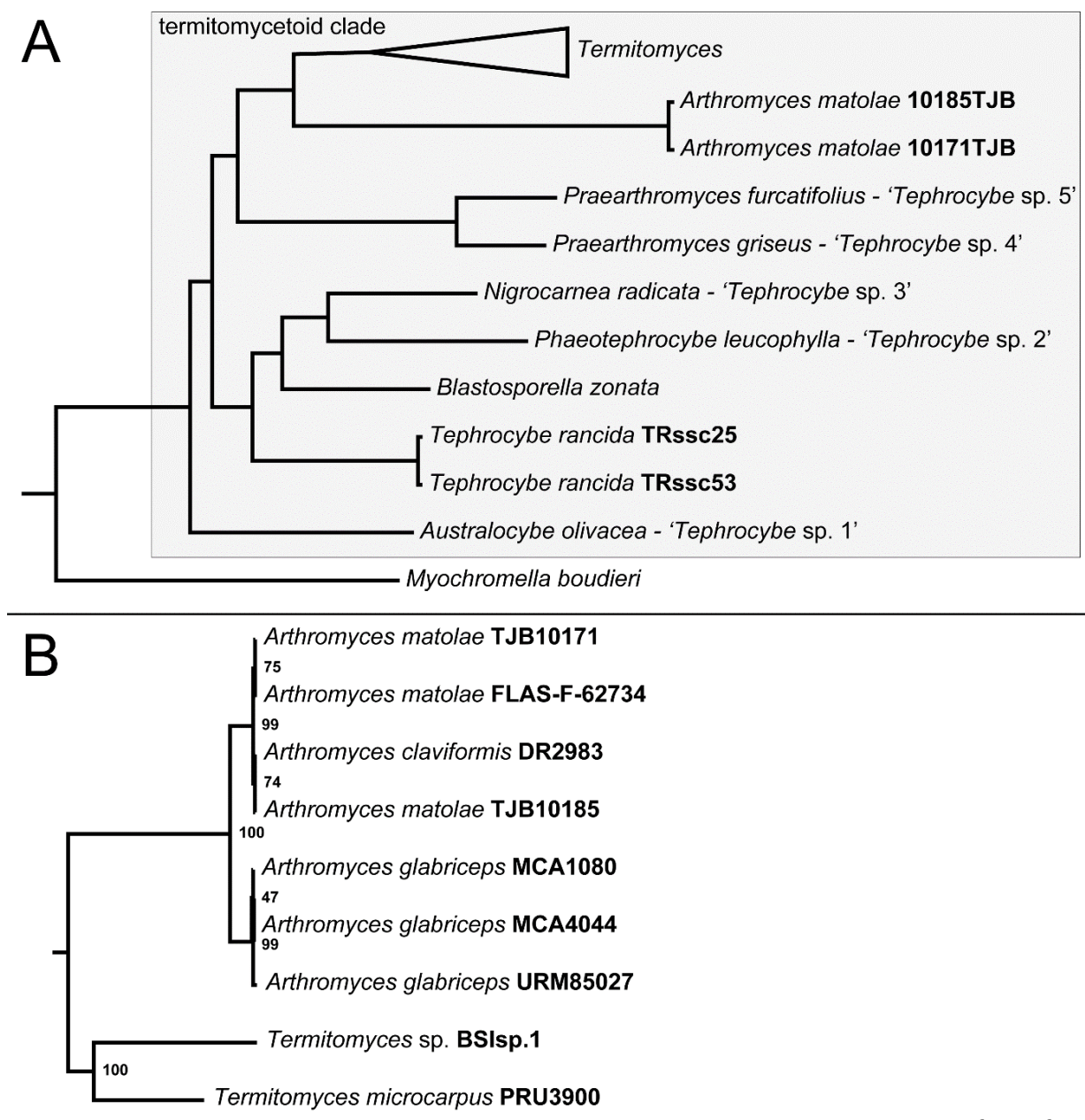

Figure $1 \mid A$ : Phylogenetic tree showing the phylogenetic placement of the five unidentified species of Tephrocybe in the termitomycetoid clade (Adapted from van de Peppel et al. 2021). B: Maximum-likelihood tree showing the novel species A. glabriceps nests with other species of Arthromyces. Numbers at the nodes indicate ultrafast-bootstraps.

\section{Taxonomy}

Australocybe olivacea T.J. Baroni \& L.J.J. van de Peppel, gen. et sp. nov.

Etymology: Australocybe - named after the continent of origin. Olivacea - named after the olive-green colour of the lamellae. 


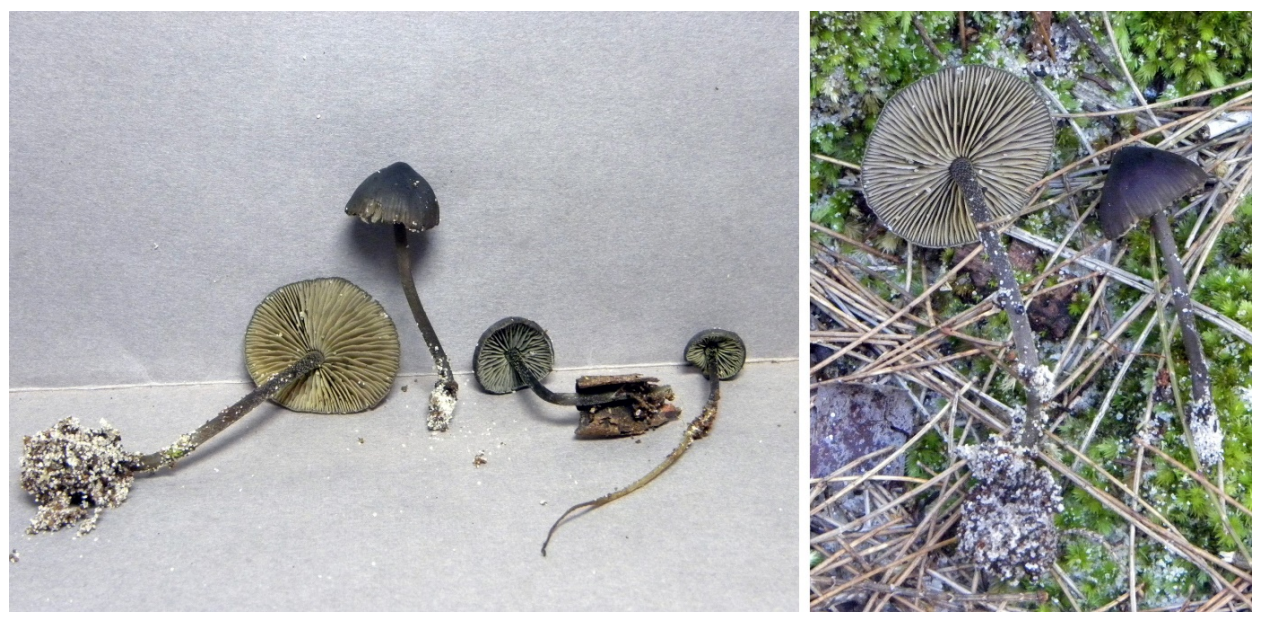

Figure 2 | Basidiomes of Australocybe olivacea, T.J. Baroni 10521

Diagnosis: Tephrocyboid with the habit of $T$. rancida but with a dark olive-grey colour of the pileus, olive-green lamellae and with both cheilo- and pleurocystidia (with resinous contents).

Description: Basidiomes dark fuscous overall except for olive-grey lamellae. Pileus 7-20 mm, broadly conical-convex becoming plane with a low rounded umbo, faintly translucently striate near margin when moist, mostly opaque and densely matted fibrillose textured when young, somewhat granular over the disc, becoming glabrous, dark fuscous brown (6-8F3, Negro), fading to grayish brown (6D-E3, Café-auLait) or brownish gray (6D2), hygrophanous and becoming a paler brownish grey (5D2, Dust) from disc out radially to margin. Lamellae olive to yellowish grey (3D2-3, Yellowish Grey) or olive brown (4C-D34, Flaxen, Sallow; 4E3), adnexed, crowded, rarely forked, broad (2.5-5 $\mathrm{mm}$ ), edge even, concolorous. Stipe concolorous with pileus, strongly tapered into a long pseudorhiza, which is a pale greenish golden-olive or greyish yellow (4B-C4, Champagne to Blonde), 1-2 x 15-45 mm, some reaching $60 \mathrm{~mm}$ long with well-developed pseudorhiza, with golden olive pruina from apex to mid stipe, glabrous below, hollow and white stuffed in medulla.

Microscopical description: Spores 6.0-7.0 x 3.0-4.0 $\mu \mathrm{m}$, ellipsoid, smooth, round in polar view, with slightly congophilous wall, walls strongly cyanophilic. Basidia 20-25 x 6-7 $\mu \mathrm{m}$, 4-sterigmate, with large cyanophilic bodies. Cheilocystidia abundant (lamella edge sterile), subglobose to ovate, pedicellate, towards apex often broadly to narrowly rostellate, 30-40 x 12-16 $\mu \mathrm{m}$, often with resinous exudation at apex, sometimes completely filled with golden brown resinous matter 
(somewhat like chrysocystidia when the material is still inside the cystidium). Pleurocystidia scattered, as cheilocystidia, often with golden brown pigment. Hymenophoral trama with hyaline, $7 \times 5 \mu \mathrm{m}$ broad, arthroconidia, sometimes attached to terminal hyphae of hymenophoral trama and there forming as arthroconidia; hymenophoral trama regular, hyphae thin-walled, with abundant cyanophilic bodies. Clamp-connections throughout in hymenophoral trama

Additional Material examined: Australia, Queensland State, Great Sandy National Park, Cooloola Sector, Freshwater Track, in sandy soil along the road side, in the same general area as CORT014480, under Allocasuarina, Eucalyptus pilularis and other Eucalyptus species, and Leptospermum, 2556'46.6"S 15307'42.6"E, 144 m elevation, 25 May 2011, T.J. Baroni 10527 (CorT014479) consisting of two basidiomata.

Nigrocarnea radicata O.S. Pedersen \& T. Læssøe gen. et sp. nov. Mycobank:

Etymology: Nigrocarnea - referring to the black flesh in the pileus. Radicata - referring to the long pseudorhiza

Diagnosis: Tephrocyboid with the habit of $T$. rancida, but with striking black, pulverulent pileus flesh consisting of chains of smooth, darkcoloured arthroconidia and the absence of clamp connections in all tissues.

Description: Small group rooting in soil under Fagaceae in rich broadleaved forest. Pileus brownish grey, ca. 7F2, with discrete broad umbo to sub-conical, minutely pruinose, margin pale grey, inrolled, 16$25 \mathrm{~mm}$, dry. Lamellae crowded, adnexed, lead grey, ca 14B1, edge slightly paler, eroded, L: 55+, l: 3, forks quite common, $1.5 \mathrm{~mm}$ broad. Pileus context deep black, 3-4 mm thick. Stipe solitary or joined in pairs with very long, very thin pseudorhiza, 16-30 × 2-4 mm excluding pseudorhiza, pruinose and fibrillose, grey, much paler than pileus. Smell and taste somewhat rancid but not very strong. Spore deposit not observed.

Microscopic description: Basidiospores few, ellipsoid, inamyloid, cyanophilous, 4.4-7.9 × 3.0-5.0 $\mu \mathrm{m}$, av. $5.4 \times 3.7 \mu \mathrm{m}$, Qav. $1.48(\mathrm{~N}=50)$. Basidia 4-spored, clavate with siderophilous granules, $15-20 \times 6-9 \mu \mathrm{m}$ $(\mathrm{N}=10)$. Hymenophoral trama regular, hyphae 3-12(-18) $\mu \mathrm{m}$ broad, hyaline, thin-walled to slightly thick-walled. Pileipellis completely 
covered by arthroconidia; hyphae a cutis, brownish, with parietal brownish pigment and some weakly encrusted; pileocystidia absent. Stipitipellis a cutis of smooth hyphae, 5-9 $\mu \mathrm{m}$ broad; without caulocystidia. Clamp connections absent at all septa. Arthroconidia in pileus trama roundish to squarish, smooth, pale-brown to grey-brown, 6.6-9.2 $\times$ 6.0-7.0 $\mu \mathrm{m}$, av. $8.3 \times 6.8 \mu \mathrm{m},(\mathrm{N}=20)$, single or in chains of maximum four conidia.
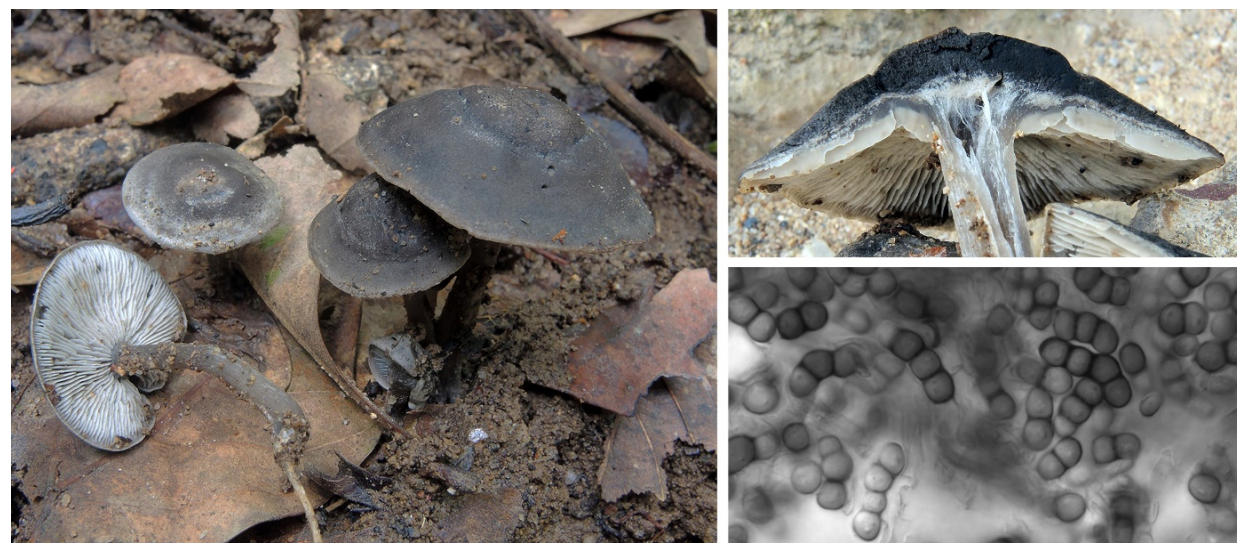

Figure 3 | Basidiomes of $N$. radicata in situ (left). B Cross section of the pileus showing the mass of dark arthroconidia (top right). Microscopic image of the arthroconidia of the pileus (bottom right).

Notes: Molecular data shows that $N$. radicata is closely related to Blastosporella zonata (Figure 1). Morphologically they look similar as they both produce conidia on the pileus and have a rooting stipe. They can be distinguished by the type of conidia as $N$. radicata produces chains of arthroconidia and $B$. zonata produces ornamented blastoconidia in small sporeheads. These two species have no overlap in geographic habitat as N. radicata occurs in the palaeotropics while $B$. zonata occurs in the neotropics. An ITS sequence generated from collection UDB034575/HNL501934 shows that it is identical to the type collection. These collections were made some $10 \mathrm{~km}$ apart in the same area, forest type and altitude and with similar macroscopic and microscopic descriptions.

Discussion: Because of the reported insect-faecal association in the closely related B. zonata (van de Peppel et al. 2021), we attempted to dig down to find the nutrient substrate to which the pseudorhiza was attached, but it was not possible to follow the very thin strand deeper than about $10 \mathrm{~cm}$ in hard soil. The type locality was very rich in ectomycorrhizal fungi and also in species of Termitomyces. 


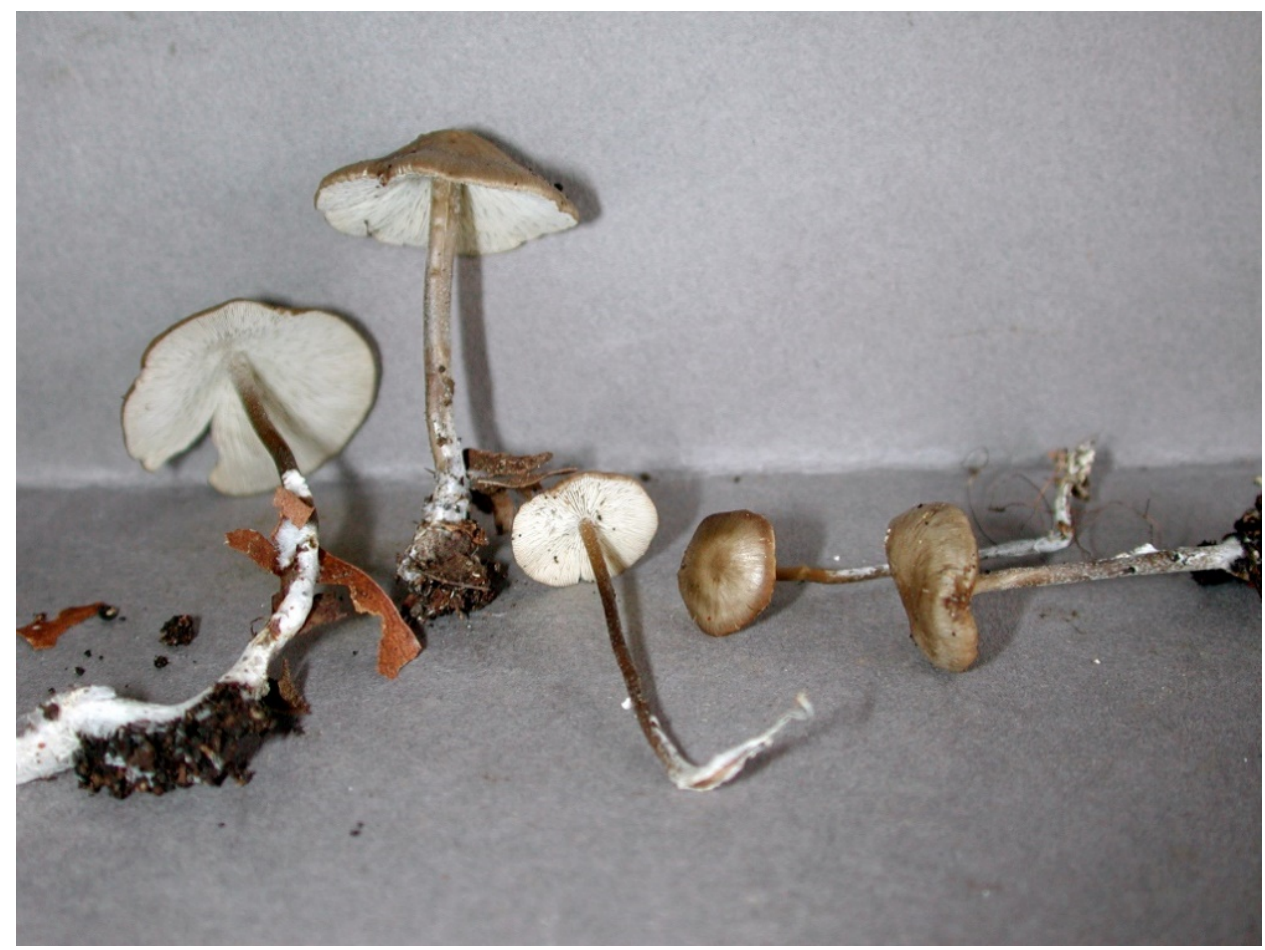

Figure 4 | Basidiomes of Ph. leucophylla, T.J. Baroni 9174

Phaeotephrocybe leucophylla T.J. Baroni, T.W. Kuyper \& L.J.J. van de Peppel, gen. et sp. nov.

Etymology: Phaeotephrocybe - referring to the brown colour of the pileus and stipe and the similarity to the genus Tephrocybe. Leucophylla - referring to the bright white lamellae.

Diagnosis: Tephrocyboid similar in appearance to $T$. rancida but easily distinguished by the brown colour of the pileus and stipe and the white gills. It can be distinguished from B. zonata, N. radicata and Arthromyces by the absence of dark blasto- or arthroconidia on the pileus.

Description: Pileus 10-25 mm, convex or broadly campanulate, some broadly truncate umbonate, moist, hygrophanous, subzonate or zonate bevelled ridged on some, pale olive yellowish brown at first (4B4 Greyish Yellow, Champagne), becoming dark gray brown (5D-E4 Dark Blonde, Hair Brown), strongly hygrophanous then shiny and paler,. Lamellae extremely crowded, adnate, white or some becoming grayish $\tan$ (5C3 brownish orange)with age, $1 \mathrm{~mm}$ broad, dichotomously forked 2-3 times, mainly when close to stipe. Stipe 30-50 mm long, 1-2.5 mm 
wide, equal, terete, mostly tapering at base to a bent short pseudorhiza, rather tough and pliant, concolorous with pileus, densely white floccose matted over base and especially over the bent pseudorhiza, fine white appressed fibrillose over the above ground portions, but especially noticeable over the upper $1 / 2$ of the stipe, hollow. Odour rancid farinaceous when crushed. Taste farinaceous fabaceous.

Microscopic description: Spores 6.0-8.0(-10) x 3.5-4(-5) $\mu$ m, elliptical or narrowly elliptical or somewhat elliptical-cylindrical in profile and face views, round in polar view, smooth, with slightly thickened, congophilous and cyanophilic wall. Basidia very short, (12-)15-20 x 5-7 $\mu \mathrm{m}, 2$ and 4-sterigmate, clavate, with cyanophilic bodies. Lamella edge sterile, consisting of cheilocystidia / cystidioles, 20-25 x 3-7 $\mu \mathrm{m}$, partly narrowing near apex, straight to somewhat flexuose, hyaline, with thin to slightly thickened walls. Pleurocystidia scattered, more or less similar to cheilocystidia or somewhat broader, 20-30 x 4-6(-8) $\mu \mathrm{m}$. Hymenophoral trama regular, consisting of parallel hyphae, with thin to slightly thickened wall. Pileus context composed of radially arranged, hyaline, mostly inflated hyphae, $8-20 \mu \mathrm{m}$ in diam. Pileipellis a cutis of brown or pale yellow brown repent cylindrical or slightly inflated hyphae hyphae, 3-6(-8) $\mu \mathrm{m}$ in diameter, pale brown pigment mainly parietal, but occasionally minutely encrusting. Stipitipellis a cutis of repent, parallel, colorless hyphae, 2.5-5 $\mu \mathrm{m}$ in diam, producing scattered clusters of cylindrial, erect and interwoven or single caulocystidia, 15-55 x 3-5 $\mu \mathrm{m}$. Clamp connections absent in all tissues. Arthroconidia absent from all tissues.

Notes: This species lacks clamp connections a feature shared with $N$. radicata, but different from $B$. zonata and $T$. rancida which have clamp connections. Although the habit of this species is similar to T. rancida, molecular data shows that it is sister to $N$. radicata (Figure 1).

Praearthromyces T.J. Baroni, T.W. Kuyper \& L.J.J. van de Peppel gen. nov.

Etymology: Prae Arthomyces - before Arthromyces.

Diagnosis: Species with tephrocyboid or collybioid habit with crowded lamellae, clampless hyphae, with a thin, deeply rooting pseudorhiza. 
Praearthromyces furcatifolius T.J. Baroni, T.W. Kuyper \& L.J.J. van de Peppel, sp. nov.

Etymology: Furcatifolius - with forked lamellae

Description: Pileus 1-2.5 $\mathrm{cm}$ wide, applanate to concave or slight infundibuliform, disc depressed or some subumbilicate, margin undulate, smooth, surface strongly velutinous but becoming densely pruinose when older and staining fingers when handled with olive gray dusty conidia when touched, concentrically zoned white, dark and light gray, the darker zones covered with chains of arthroconidia. Contex dull white or pale gray, soft. Lamellae densely crowded, narrow (less than 1 $\mathrm{mm}$ ), often forked or bifurcate near stipe attachment but also in the middle or near the pileus edge, pale gray or gray, edges white and fimbriate and then finely eroded. Stipe 5-13 cm long, 1-3 mm wide, cylindrical or compressed and twisted, gradually tapering downwards to a long pseudorhiza, fasciculate strongly at the base, not easily separated, pale to dark gray, pale grayish over the pseudorhiza, surface strongly pruinose on above ground parts.

Microscopic description: Basidiospores 5-6(-7) x (3-)4-4.5 $\mu \mathrm{m}$, not very abundant, ellipsoid in profile and face views, round in polar view, smooth, hyaline, only slightly congophilous wall, but walls cyanophilic. Basidia (12-)15-20 x 5-6 $\mu \mathrm{m}$, apparently 2-sterigmate, sterigma difficult to confirm, but whole mounts of lamellae in polar view show four spores over the basidia/basidioles indicating 4-sterigmate basidia as well, but this number could not be confirmed viewing individual basidia/basidioles, basidia clavate or narrowly clavate, with cyanophilic bodies. Cheilocystidia cylindrical-contorted or diverticulate, often branched and knobby, also some subclavate, irregularly septate, producing a thick layer of interwoven, often collapsed end cells, difficult to observe individual cells. Pleurocystidia absent. Lamella trama composed of olive brown inflated hyphae, similar in shapes and size to those of the pileus context. Pileus context pale olivaceous brown, radially arranged, mostly inflated hyphae, (6-)10-24 $\mu \mathrm{m}$ in diam, also with scattered narrowly cylindrical dark brown encrusted hyphae mixed in. Pileipellis a pale or dark brown layer of repent cylindrical hyphae producing a cutis of hyphal cells, 2.5-5.5 $\mu \mathrm{m}$ in diameter, with brown parietal pigments and distinctly darker colored septa; producing erect end cells with straight or often curved hyaline hyphae that eventually form septa and chains of thick-walled brown ornamented arthroconidia, up to 10 cells in length but frequently 5-7 cells remain connected, individual conidia ranging from subglobose to broadly ellipsoid, 7-9 $x$ 
5.5-7 $\mu \mathrm{m}$, some conidia becoming elongate ellipsoid and slightly curved phaseoliform, up to 10-13 x 5.5-7 $\mu \mathrm{m}$, thick-walled, dark yellowish brown, with obvious verrucose ornamentation. Clampconnections absent in all tissues.

Notes: Species in this genus closely resemble Arthromyces by having a pseudorhiza and in the case of Pr. furcatifolius by producing chains of dark arthroconidia on the basidiome. However, phylogenetic reconstruction shows a sister relationship of Praearthromyces to the Arthromyces-Termitomyces clade (Figure 1)(van de Peppel et al. 2021). Placing Pr. furcatifolius in the genus Arthromyces would make the latter genus paraphyletic or would require Arthromyces to be synonymized with Termitomyces, which is not warranted because of the lack of a termite association. The genus can be distinguished from Australocybe, Blastosporella and Tephrocybe by the lack of clamp connections, a feature that is shared with Arthromyces, Phaeotephrocybe, Nigrocarnea and Termitomyces. It can be further distinguished from Termitomyces by the absence of a termite association. Currently, the genera Praearthromyces and Arthromyces can only be distinguished by either their geographic origin, Arthromyces from the new world and Praearthromyces from the old world or by DNA sequence data.

Discussion: The study by van de Peppel et al. (2021) showed that this taxon is part of a two species clade sister to the ArthromycesTermitomyces clade (Figure 1). Corner (1994) described Tricholoma furcatifolium, characterized by very crowded, narrow, regularly furcate lamellae (Corner 1994). The description does not refer to the production of dark arthroconidia on the basidiome. We were unable to obtain a sequence from the holotype. However, another collection made by Corner under the name $T$. furcatifolium did produce arthroconidia. We managed to obtain an ITS sequence from this collection; this sequence was $99 \%$ identical to the sequence we obtained from the DS06151 collection. We are unable to resolve whether the holotype and this second collection made by Corner represent different species or morphological variation within a species. In order to prevent future nomenclatural issues related to the holotype of Tricholoma furcatifolium, we describe this new species in a new genus with the epithet furcatifolius, thereby pre-empting any future transfer of Corner's holotype to this genus (Art. 11.4 of the International Code of Nomenclature for Algae, Fungi and Plants). 


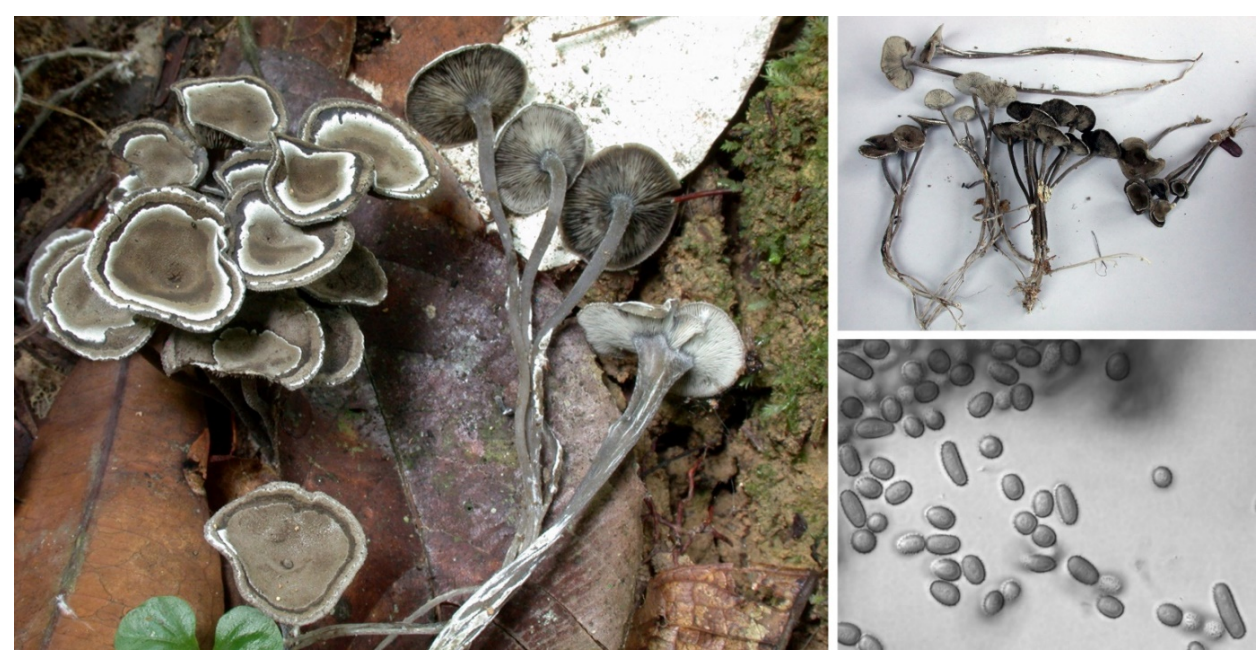

Figure 5 | Basidiomes of Praearthromyces furcatifolius, DS06151. Microscopic image of the arthroconidia produced on the pileus (bottom right).

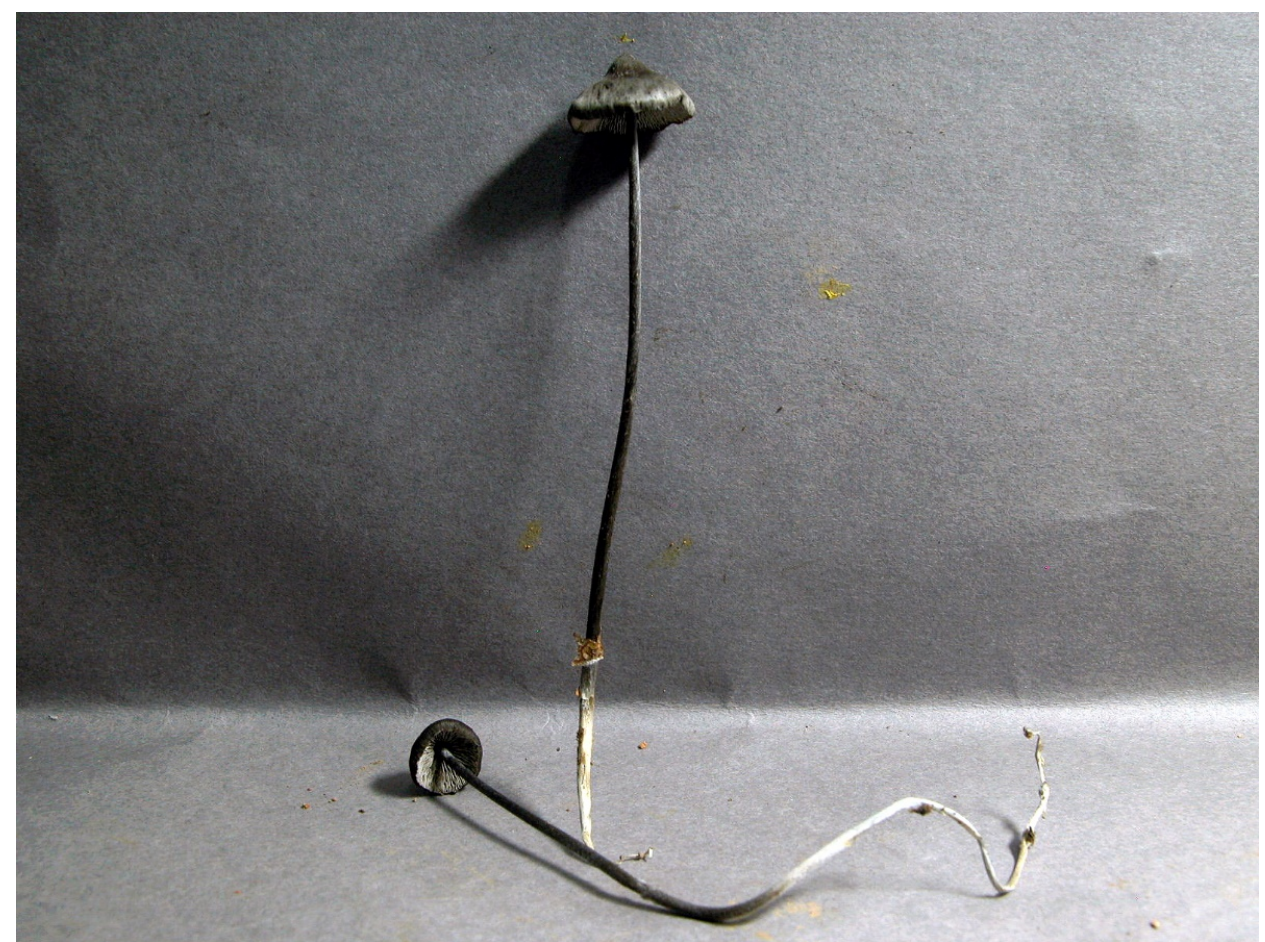

Figure 6 | Basidiomes of Pr. griseus, T.J. Baroni 9980 
Praearthromyces griseus T.J. Baroni \& L.J.J. van de Peppel, sp. nov.

Etymology: Griseus - referring to the grey colour of the pileus

Diagnosis: Tephrocyboid habit similar to $T$. rancida but with cheilocystidia and the absence of clamp connections.

Description: Pileus 8-14 mm broad, conical with broad conical umbo or narrowly campanulate, margin incurved, dry, with radiate innate fibrillose surface, deep grayish with brown hues (5F3-4 Nutria, Sepia, Beaver; to 5E3 Drab or Mouse Grey). Context pale grayish, thin (less than $0.5 \mathrm{~mm}$ ). Lamellae deeply adnexed, crowded, edges eroded and fimbriate, broad (1 mm), pale grayish or brownish gray (5C-D2 Birch Gray, Nougat). Stipe 8-9.5 cm long, 1-1.5 mm broad, equal, but strongly tapered into a long sometimes curving pseudorhiza, terete, fine white pruinate over apex, fine silvery white scattered appressed fibrillose below, concolorous with pileus (5E3 Drab or Mouse Grey), except for white contrasting subterranean pseudorhiza, narrowly hollow and grayish in context. Odor faintly farinaceous when cut.

Microscopic description: Basidiospores 6-9(-10) × 3-4.5 $\mu \mathrm{m}$, ellipsoid or ellipsoid elongate in profile and face views, round in polar view, walls cyanophilic and congophilous. Basidia (17-) 20-30 x (5-)6-8 $\mu \mathrm{m}, 4$ sterigmate, with small and sometimes sparse cyanophilic bodies. Cheilocystidia densely crowded along lamella edge, hyaline, cylindrical but also some sublageniform, frequently undulate over upper $1 / 2$ of cylindrical neck, 18-32(-55) × 3-6 $\mu$ m. Pleurocystidia absent. Lamella trama pale brown from parietal pigment, composed of parallel cylindrical or inflated hyphae, 4-15 $\mu \mathrm{m}$ in diam. Pileus context similar in color and hyphal make-up to lamella trama, pale brown from parietal pigment, radially arranged, inflated hyphae mixed with cylindrical hyphae, 5-20 $\mu \mathrm{m}$ in diam. Pileipellis two layered, with a thin hyaline layer of a few cylindrical hyphae, 2-4 $\mu \mathrm{m}$ diam, overlying a dark brown layer of hyphae that have parietal and clearly encrusting pigments on many of these gradually larger hyphae, 4-13 $\mu \mathrm{m}$ in diam, as one progresses into the context. Stipitipellis similar to the pileipellis, with a thin hyaline layer of cylindrical hyphae, 2-4 $\mu \mathrm{m}$ in diam, overlying a pale brown layer of progressively broader cylindrial or slightly inflated hyphae, 5-15 $\mu \mathrm{m}$ in diam, with parietal pigments. Clamp connections absent in all tissues. Arthroconidia not present.

Notes: Pr. griseus can easily by distinguished from Pr. furcatifolius by the lack of arthroconidia on the basidiome and lamellae that are not forked 
Arthromyces glabriceps M.C. Aime \& Coimbra sp. nov.

Etymology: glabri-ceps Latin, for the glabrous pileus

Diagnosis: Similar to A. matolae, but differing in the lack of conidia on the pileus.

Description: Pileus up to $4 \mathrm{~cm}$ diameter, glabrous, hygrophanous, concentrically grooved, plane with broad umbo, brown 6F5 to 5E5, margin paler, occasionally uplifted, translucent-striate; umbo flat or conic, subtended by a nipple, shallowly corrugated under hand lens, paler than pileus. Lamellae narrow, crowded, L: ca. $60 \mathrm{~mm}$, pallid $3 \mathrm{~A} 2$ to 5A2 then mottled. Stipe: hollow, taupe to greyish, 3D2, white fibrillose under hand lens, tomentum dense, white, even, with short, narrow tapered pseudorhiza, 79-180 $\mathrm{mm} \times 3 \mathrm{~mm}$.

Microscopic description: basidiospores (5-)6-7(7.5) x 3-4 $\mu \mathrm{m}$, ellipsoid in profile and face views, round in polar view, smooth, often biguttulate, walls cyanophilic, inamyloid. Basidia 2-sterigmate, 15-20 x 5$8 \mu \mathrm{m}$, clavate, with cyanophilic bodies, basidia scattered on lamella margins. Cheilocystidia 29-52 × 2-4 $\mu$ m, hyaline, cylindrical, crowded into coagulated fasciculate clusters along the lamella edge, and covered by deep golden or golden brown encrusting resinous pigments often making the individual cystidia difficult to discern. Pleurocystidia absent. Lamella trama hyaline, composed of parallel cylindrical or inflated hyphae, 5-22 $\mu \mathrm{m}$ in diam. Pileus context hyaline or pale brown, composed of radially arranged, mostly inflated hyphae, 10$30 \mu \mathrm{m}$ in diameter, with many short barrel shaped cells. Pileipellis a deep (80-100 $\mu \mathrm{m}$ in depth), hyaline layer, composed of a cutis of repent cylindrical, loosely interwoven hyphae, 2-4 $\mu \mathrm{m}$ in diam. Stipitipellis hyaline, repent layer, cylindrical, 2-5(-7) $\mu \mathrm{m}$ in diam. Clamp connections absent in all tissues. Arthroconidia absent in all tissues.

Notes: Arthromyces glabriceps differs from $A$. matolae and $A$. claviformis in lacking the production of conidia on the pileus, and in its distribution; A. glabriceps is apparently restricted to the Guiana Shield, known only from the western Pakaraima Mountains of Guyana, and from Brazil.

Distribution and habitat: In Dicymbe corymbosa monodominant forest or adjacent mixed forest; despite multiple years of collecting effort in 
this region, known only from the Upper Potaro River Basin of Guyana and Brazil.
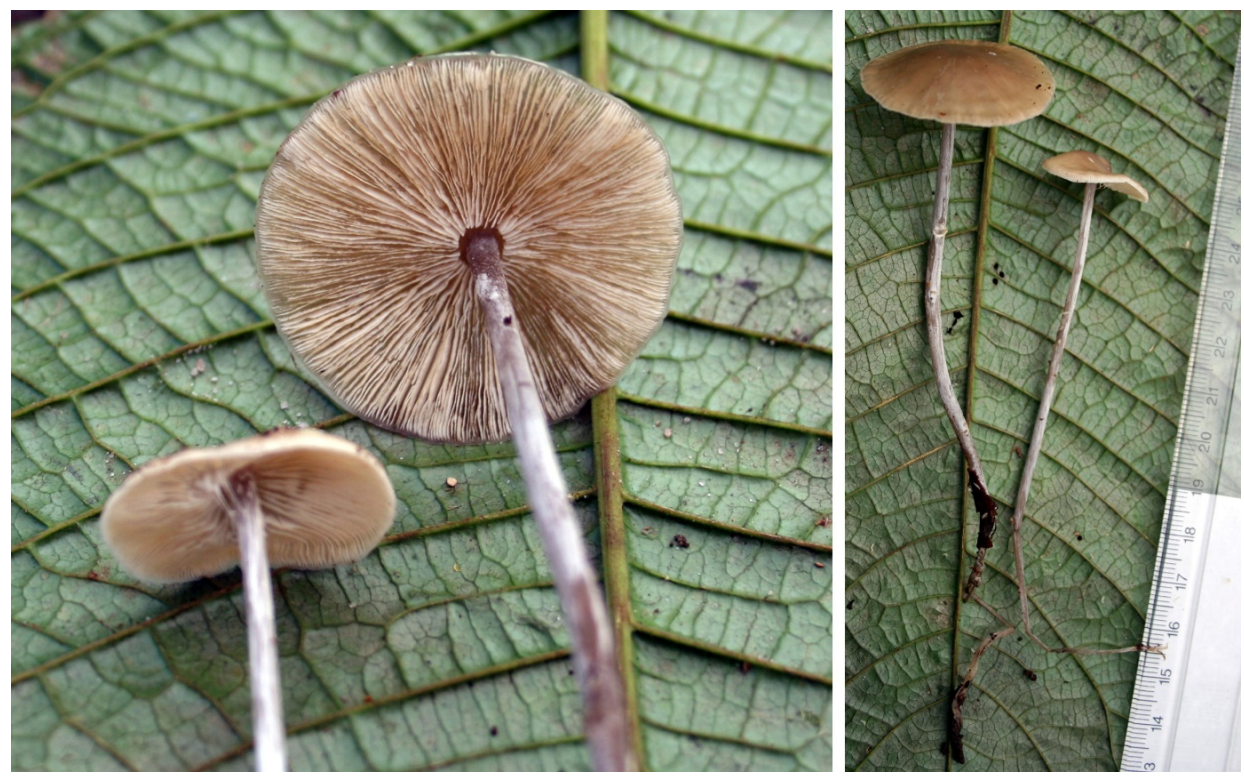

Figure 1 | Basidiomes of $A$. glabriceps, MCA4044

Additional material examined: Guyana: Region 8 Potaro-Siparuni, Pakaraima Mountains, Upper Potaro River Basin, approximately $15 \mathrm{~km}$ east of Mt. Ayanganna, vicinity of Potaro base camp, elev. 710-750 m, 518'04"N 5954'40"W, M.C. Aime 1080 (BRG; PULxxx); M.C. Aime 1297 (BRG; PULxxx); 2002 Jul 03, M.C. Aime 2194A (BRG; PULxxx); 2002 Jul 03, M.C. Aime 2194B (BRG; PULxxx); M.C. Aime 3988 (BRG; PULxxx); 2010 May 27 (BRG; PULxxx); M.C. Aime 4044 (BRG; PULxxx); M.C. Aime 4318 (BRG; PULxxx); 2012 May 31 M.C. Aime 4784 (BRG; PULxxx); 2016 Jun 18, M.C. Aime 6216 (BRG; PULxxx).

\section{Discussion}

A recent phylogenomic study included five of our newly described species (as Tephrocybe sp1-5.)(van de Peppel et al. 2021). The study by van de Peppel et al. already showed that geography and morphological features such as conidial production, clamp connections and insectfaecal associations were not consistent with the clades recovered from their phylogeny (see also Table 1). We therefore justify the erection of the four new genera based on the six criteria as proposed by Vellinga et al. (Vellinga et al. 2015). These six criteria are: (1) monophyly; (2) sufficient phylogenetic coverage (3) sufficient branch support (4) discussion of alternative options (discussed below) (5) no single marker 
phylogeny (6) include all supporting evidence and background information.

The single species in the genus Australocybe, A. olivacea, is macro-morphologically quite similar to $T$. rancida. However, phylogenetic analysis shows that it does not nest in the same clade as T. rancida but occupies an isolated branch (van de Peppel et al. 2021). Placing this species in Tephrocybe would require synonymization of the entire termitomycetoid clade to preserve monophyly (criterium 1), in which the oldest name; Termitomyces, would take priority. This is extremely undesirable as it would include many non-termite associated species in the genus Termitomyces. A similar problem would occur if we would place the two species of Praearthromyces in Arthromyces, which in theory could be justified based on their morphology, but which would make it unavoidable that the genera Praearthromyces and Arthromyces to be synonymized with Termitomyces. Although Arthromyces grows on insect faeces and so may be insect-associated, it is highly unlikely that these insects are termites (van de Peppel et al. 2021). As mentioned previously, we consider that the (unique) termite association of Termitomyces necessitates maintaining the current definition of the genus, and therefore making erecting several new small or monotypic genera inevitable.

Table 1 | Overview of morphological characters represented by the new species and their close relatives.

\begin{tabular}{|l|c|c|c|c|c|l|}
\hline & Characters & & & & & \\
\hline & conidia & clamp & cheilo- & pleuro- & Insect-faecal & \\
\hline species & on basidiome & connections & cystidia & cystidia & association & continent \\
\hline Australocybe oliveacea & $\mathbf{0}$ & $\mathbf{x}$ & $\mathbf{x}$ & $\mathbf{x}$ & $\boldsymbol{?}$ & Australia \\
\hline Tephrocybe rancida & $\mathbf{0}$ & $\mathbf{x}$ & $\mathbf{0}$ & $\mathbf{0}$ & $\mathbf{0}$ & North America, Europe \\
\hline Blastosporella zonata & $\mathbf{x}$ & $\mathbf{x}$ & $\mathbf{x}$ & $\mathbf{0}$ & $\mathbf{x}$ & South America \\
\hline Nigrocarnea radicata & $\mathbf{x}$ & $\mathbf{0}$ & $\mathbf{x}$ & $\mathbf{?}$ & $\boldsymbol{?}$ & Asia \\
\hline Phaeotephrocybe leucophylla & $\mathbf{0}$ & $\mathbf{0}$ & $\mathbf{x}$ & $\mathbf{x}$ & $\boldsymbol{?}$ & South America \\
\hline Praearthromyces furcatifolius & $\mathbf{x}$ & $\mathbf{0}$ & $\mathbf{x}$ & $\mathbf{0}$ & $\boldsymbol{?}$ & Asia \\
\hline Praearthromyces griseus & $\mathbf{0}$ & $\mathbf{0}$ & $\mathbf{x}$ & $\mathbf{0}$ & $\boldsymbol{?}$ & South America \\
\hline Arthromyces glabriceps & $\mathbf{0}$ & $\mathbf{0}$ & $\mathbf{x}$ & $\mathbf{0}$ & $\boldsymbol{?}$ & South America \\
\hline Arthromyces matolae & $\mathbf{x}$ & $\mathbf{0}$ & $\mathbf{x}$ & $\mathbf{0}$ & $\mathbf{x}$ & South America \\
\hline Termitomyces spp. & $\mathbf{0}$ & $\mathbf{0}$ & $\mathbf{x}$ & $\mathbf{x}$ & $\mathbf{x}$ & Africa \\
\hline
\end{tabular}

The two species $N$. radicata and Ph. leucophylla share the tephrocyboid habit with $T$. rancida. Ph. leucophylla also shares the feature of not producing conidia with $T$. rancida. Both $N$. radicata and $B$. zonata produce conidia, while only the latter produces ornamented conidia. The genera Phaeotephrocybe and Nigrocarnea can be separated from the genera Blastosporella and Tephrocybe by the lack of clamp connections. If we wanted to conserve monophyly without erecting a 
new genus, both of these new species together with $B$. zonata should be placed in Tephrocybe. We consider this solution as a possible retrograde step as the variation in traits (both absence and presence of conidia, clamp connections, insect-association) within Tephrocybe would leave no synapomorphies to separate Tephrocybe from the other related genera. The genera Tephrocybe and Blastosporella are currently easily distinguished by the absence of an insect association in the former and the presence of an insect association in the latter (van de Peppel et al. 2021). Since we could not confirm an insect association in $N$. radicata and $P h$. leucophylla we chose to erect two new genera instead of placing them in Blastosporella. Placing N. radicata and Ph. leucophylla in a single genus was also not a valid option as these species do not share many morphological characters (Table 1) and branch lengths suggest significant genetic divergence between the two. Biogeographical considerations would also argue against combining both in one genus. The lack of clamp connections in these genera sets them apart from Blastosporella and Tephrocybe, while Phaeotephrocybe can be easily distinguished from Nigrocarnea by the absence of internal dark-pigmented arthroconidia.

The addition of the genera Australocybe, Phaeotephrocybe, Nigrocarnea and Praearthromyces to the Lyophyllaceae and especially the termitomycetoid clade shows that even more ecological transitions may have occurred within this group than previously thought (Hofstetter et al. 2014). Insect associations have only been demonstrated in the genera Arthromyces, Blastosporella and Termitomyces; however, the presence of a pseudorhiza and other shared characters with Termitomyces suggests that other species also could be insectassociated. The diversity and widespread geographic distribution of these genera suggests that more species await discovery. 



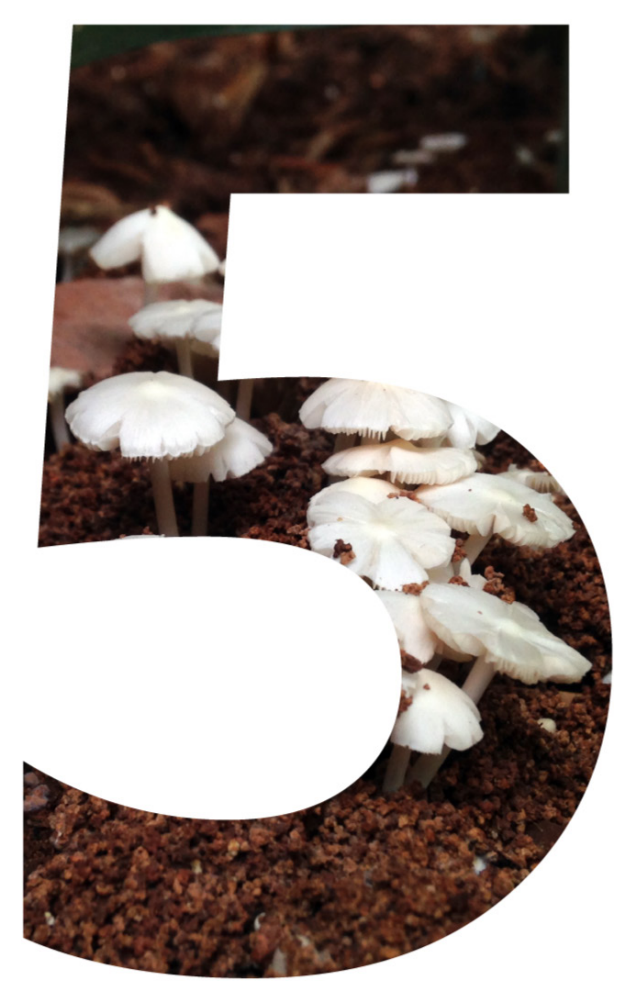

Species and speciation in the termite-cultivated fungus Termitomyces

Lennart J.J. van de Peppel

Z. Wilhelm de Beer

N'Golo A. Koné

Duur K. Aanen 


\section{Abstract}

Termitomyces is a genus of basidiomycete fungi cultivated by termites of the subfamily Macrotermitinae. This symbiosis originated in central Africa, and subsequently, the fungus-growing termites have colonized almost the entire African continent including Madagascar as well as significant parts of the Asian continent. Around 40 species of Termitomyces have been described based on morphology of the sexual fruiting bodies, which are associated with some 330 species of fungusgrowing termites distributed over 11 genera. However, the total number of fungal species may be higher as not all species regularly produce mushrooms, and morphological variation does not seem to be a reliable criterion for species delimitation in this group. In this study we estimated the total number of species based on ITS-barcode criteria and assessed host specificity and geographic differentiation to infer patterns of speciation. We estimated the total number of genetic species using Automatic Barcode Gap Discovery (ABGD) on a large dataset of well over 1500 ITS sequences from laboratory cultures, herbarium specimens and the public database NCBI Genbank. This resulted in 87 species hypotheses. A phylogenetic reconstruction was performed on representative sequences of those 87 species hypotheses constrained by a well-supported phylogeny based on whole-genome data to address host specificity and geographical differentiation. Five main clades were recovered which generally were associated with species of one or two host genera, except for samples collected from the genera Microtermes and Ancistrotermes, which formed two separate non-sister clades. We did not find any evidence for long-term host fidelity as would be expected for species with strictly vertical transmission. We found strict geographic separation between African and Asian species of Termitomyces and infer a minimum of seven inter-continental migrations. We show that epigeous fruiting of the T. microcarpus group has a single evolutionary origin in Africa and that fruiting in species of this group likely is induced by the fungus. In contrast, fruiting in the symbionts of some species of Microtermes and Macrotermes may be suppressed by the termite, as mushrooms of certain species are found in some host genera, but not in others. We discuss some examples of incongruence between morphological and genetic species concepts and give suggestions to improve taxonomy of the genus Termitomyces. 


\section{Introduction}

Basidiomycete fungi of the genus Termitomyces (family: Lyophyllaceae) are cultivated by species of a subfamily of termites, the Macrotermitinae. The symbiosis has a single origin around 30 million years ago in the rain forests of central Africa (Aanen et al. 2002, Aanen and Eggleton 2005, Roberts et al. 2016). Since all extant species of Termitomyces form a clade, only a single successful domestication event occurred and no reversals to a free-living state have been reported (Aanen et al. 2002, van de Peppel et al. 2021). From central Africa fungus-growing termites expanded their range through subSaharan Africa, including a migration of the genus Microtermes to Madagascar (Nobre, Eggleton, and Aanen 2010). Four of the eleven described genera of fungus-growing termites have been established in parts of Asia (Bignell, Roisin, and Lo 2010).

Fungus-growing termites cultivate Termitomyces on a structure called the fungus comb which is constructed from primary faeces. On the fungus comb Termitomyces produces small asexual structures that are called nodules. The nodules are consumed by the termites and several different functions have been attributed to them. One of these functions is efficient within-nest propagation of the fungus; upon consumption the conidia present in the nodules are mixed with the plant material in the gut, and after a short gut passage, these primary faeces are used to construct the fungus comb (Leuthold, Badertscher, and Imboden 1989). The nodules are also thought to play a role in nutrition by providing the termites with a reliable protein source and the essential amino acid tryptophan (Nobre and Aanen 2012, Chiu et al. 2019). The fungus may also provide the termites with additional digestive enzymes (Martin and Martin 1978).

Basidiocarps (mushrooms) are observed for the majority of fungus-growing termite species, which rely on the basidiospores for inoculation of the fungus in newly established colonies (Johnson et al. 1981, Korb and Aanen 2003, de Fine Licht, Boomsma, and Aanen 2006). In only two independent cases vertical transmission of the symbiont has evolved: via the male reproductive of the species Macrotermes bellicosus and via the female reproductive of all studied species of the genus Microtermes asexual fungal spores are carried in the gut (Johnson et al. 1981, Johnson 1981, Korb and Aanen 2003). The production of mushrooms usually occurs during the rainy season which coincides with the production of the reproductives (alates) (Koné et al. 2011, Koné et al. 2018). There is a moderate degree of host-specificity 
between fungus-growing termites and Termitomyces, mainly on a generic level (Aanen et al. 2002, Aanen et al. 2007, Osiemo et al. 2010). The genus Termitomyces was erected by Roger Heim in 1942, before which fungus-growing termite symbionts were placed in several unrelated fungal genera (Heim 1942). Currently, Termitomyces is a large genus with 100 names listed in Index Fungorum (July 2021, http://www.indexfungorum.org/). These names include all names that have been validly published and therefore also contains synonyms and different forms of a single species, which makes it unclear how many biological species are represented. Large differences in macromorphological characteristics exist within the genus, particularly in size. For example, the genus contains both T. titanicus, which is considered to be the largest mushroom in the world with a cap diameter of up to one metre, but also T. microcarpus which has a cap diameter of less than two centimetres (Pegler and Piearce 1980, Piearce 1987). For most species of Termitomyces the fruiting process starts underground; primordia are formed on the fungus comb in the termite nest and the immature basidiocarp is pushed to the soil surface by a root-like structure called a pseudorhiza. Some species also have a cap with a hard pointed umbo called a perforatorium which is thought to facilitate penetration through the soil (Heim 1977). A notable exception to this way of fruiting is the species $T$. microcarpus which fruits aboveground on comb material ejected by the termites. Probably as a response to this particular way of fruiting it does not produce a pseudorhiza and also does not have a perforatorium.

Taxonomy of Termitomyces is mostly based on macromorphological characters of the basidiocarp such as size, shape, colour of the pileus, shape of the perforatorium and even the colour of the pseudorhiza. This has led to a biased representation of the total diversity of the genus as some species are thought to rarely produce basidiocarps or even may have lost the ability to fruit (Wood and Thomas 1989, Darlington 1994, Bingham 2002). Due to current taxonomical practices many of these rarely fruiting species remain undescribed, as researchers only possess laboratory cultures made by plating fungal nodules with no representative basidiocarp (Makonde et al. 2013). Only recently $T$. cryptogamus has been described based on morphological features of a laboratory culture in combination with molecular data (van de Peppel et al. 2021, under review). Although this species has been studied extensively it remained unnamed for almost two decades (De Fine Licht, Andersen, and Aanen 2005, de Fine Licht, Boomsma, and Aanen 2006, Aanen et al. 2009, Poulsen et al. 2014). Research has also shown major geographical differentiation between 
African and Asian Termitomyces taxa (Froslev et al. 2003). This geographical differentiation has often not been considered in the naming of species of Termitomyces some names are shared between African and Asian specimens, while genetically they may be diverse (Froslev et al. 2003). These examples show that macromorphological descriptions of the mushroom should always be complemented with molecular evidence. For fungi, the ribosomal internal transcribed spacer (ITS) marker has been suggested as a barcode sequence (Schoch et al. 2012).

Here, we studied genetic diversity in the genus Termitomyces by using species delimitation software on a large dataset of ITS sequences generated specifically for this study as well as sequences from Genbank. With the species delimitation analysis we were able to estimate the number of known phylogenetic species. We combined our species hypotheses with metadata on location and termite host species to arrive at an improved species concept for Termitomyces. Using this improved species concept and further resolved intrageneric relationships we evaluated the importance of five factors which could potentially influence diversification and speciation in the genus Termitomyces: host-specificity, geographic separation, symbiont transmission mode, host control over fruiting and fruiting mode (hypogeous versus epigeous). Our species hypotheses should offer a framework for future taxonomists and may facilitate future taxonomical practices in Termitomyces as it offers diagnostic features other than basidiocarp-based morphological differences.

\section{Methods}

\section{Taxon sampling}

Sequences generated for this study came from two major sources, the first being our in-house collection which consists of a culture collection of Termitomyces strains collected in South Africa and ethanol-preserved nodules and mushrooms collected from various places in central and western Africa. The second source were herbarium samples from four different herbaria: Naturalis Biodiversity Centre, the Netherlands, Royal Botanic Gardens KEW, United Kingdom (K), Meise Botanic Garden, Belgium (BR) and two herbaria in South Africa: the South African National Collection of Fungi (PREM) and the Schweickerdt Herbarium (PRUM). To link names to some of our species hypotheses, material of several type specimens was collected.

In addition to the sequences that were specifically generated for this study we obtained additional ITS sequences from Genbank. We 
used the following search filters: "Termitomyces", "Lyophyllaceae" and "internal transcribed spacer". Metadata such as species name, collection location, termite host species and isolation source were also extracted and put in a spreadsheet. To filter out any mislabelled sequences we did an initial sequence alignment and phylogenetic tree reconstruction (data not shown). We checked sequences on long branches using NCBI Blast. This method was sufficient to filter out sequences of ascomycetes or non-Termitomyces basidiomycetes. In a few cases unreliable sequences were removed after manual inspection as some sequences had multiple mutations in the conserved $5.8 \mathrm{~S}$ region. Additionally, to name more of our species hypotheses we added sequences from the online UNITE database (https://unite.ut.ee/). In this curated database species hypotheses are generated using sequence similarity thresholds of the ITS region (Abarenkov et al. 2010). Most of these species hypotheses are reliable identifications as sequences are linked to a herbarium or voucher specimen. A sequence of Blastosporella zonata and Arthromyces matolae were added as outgroup species.

\section{DNA isolation and PCR}

For DNA extraction, a small piece of tissue of about $0.1-0.5 \mathrm{~g}$ of either a laboratory culture, basidiocarp or dried herbarium specimen was used. DNA was extracted following cetyltrimethylammonium bromide (CTAB) protocol used in a previous study (Nieuwenhuis et al. 2019). As DNA from old herbarium material is largely degraded we aimed to increase the DNA yield by extending the precipitation step in our protocol to two weeks for the herbarium samples (Staats et al. 2011).

An initial PCR was done to test whether the ITS marker could be amplified in its entirety using either the fungus-specific ITS1f (Gardes and Bruns 1993) or Termitomyces-specific ITS1fT forward primer (Aanen et al. 2007) and the universal reverse ITS4 primer (White et al. 1990). In case the ITS marker could not be amplified, ITS1 and ITS2 were amplified separately by using the ITS1fT and ITS2 primers for ITS1 and the ITS3 and ITS4 primers for ITS2 (White et al. 1990). In case amplification was still unsuccessful another attempt was conducted using ITS1fT as forward primer and a newly designed Termitomycesspecific reverse primer; ITS2T (AGATCCGTTGCTGAAAGTTG) for ITS1. For ITS2 the newly designed Termitomyces-specific forward primer ITS3T (AGTGTCATTTAAATTCTCAACC) and the general reverse primer ITS4 were used (White et al. 1990). The program used for all PCR reactions was as follows: initial denaturation at $94^{\circ} \mathrm{C}$ for five minutes, followed by 35 cycles consisting of one minute of denaturation at $94^{\circ} \mathrm{C}$, 
one minute of annealing at $53^{\circ} \mathrm{C}$ and elongation for one minute at $72^{\circ} \mathrm{C}$, followed by a final extension step for 10 minutes at $72^{\circ} \mathrm{C}$.

\section{Species delimitation}

Combining our set of newly generated ITS sequences and the filtered set of ITS sequences from Genbank resulted in a dataset consisted of a total of 1577 sequences. We used Automatic Barcode Gap Discovery (ABGD) with default settings for species delimitation (Puillandre et al. 2012).

\section{Phylogenetic reconstruction}

For each species hypothesis we extracted a single representative sequence, this was either the longest or most complete sequence. The sequences were aligned using the web server of MAFFT (7.475) using an iterative refinement strategy (FFT-NS-i)(Katoh and Standley 2013).

After trimming the alignment we conducted phylogenetic reconstruction using RAxML version 8.2.12 (Stamatakis 2014) with 100 bootstraps using a GTR+gamma model, species 69 as the outgroup and the tree from van de Peppel et al. 2021 (in prep.) as a constraint tree.

\section{Fungus-growing termite phylogeny}

In order to reconstruct a phylogeny on fungus-growing termite hosts, we downloaded all available fungus-growing termite complete mitochondrial genomes in Genbank (September, 2020). We also added three complete mitochondrial genomes of Sphaerotermitinae and used Labritermes buttelreepeni as an outgroup (Bucek et al. 2019). We extracted all 14 mitochondrial core genes and the 12S rRNA and 16S rRNA and made individual alignments for each gene using Geneious 10.0.9 (Kearse et al. 2012). Individual alignments were concatenated using Geneious and phylogenetic reconstruction was conducted using RAxML version 8.2.12 (Stamatakis 2014) with 100 bootstraps using a GTR+gamma model and Labritermes buttelreepeni as the outgroup. Partitioning by gene did not result in better support, so for the final analysis only the third codon position was excluded.

\section{Results}

\section{Species delimitation}

Our species delimitation analysis resulted in 89 species hypotheses (87 species of Termitomyces and two outgroup species), which was the most conservative estimation. 


\section{Type specimens}

We obtained 20 type specimens from herbarium collections, and for 11 of these we obtained either a full ITS or partial ITS sequence, this included two specimens older than 140 years (Table 1).

Table 1 | Overview of type specimens collected and sequenced with their corresponding herbarium or Genbank identification number, type status, part of ITS sequenced, species hypothesis number, continent of origin and collection year.

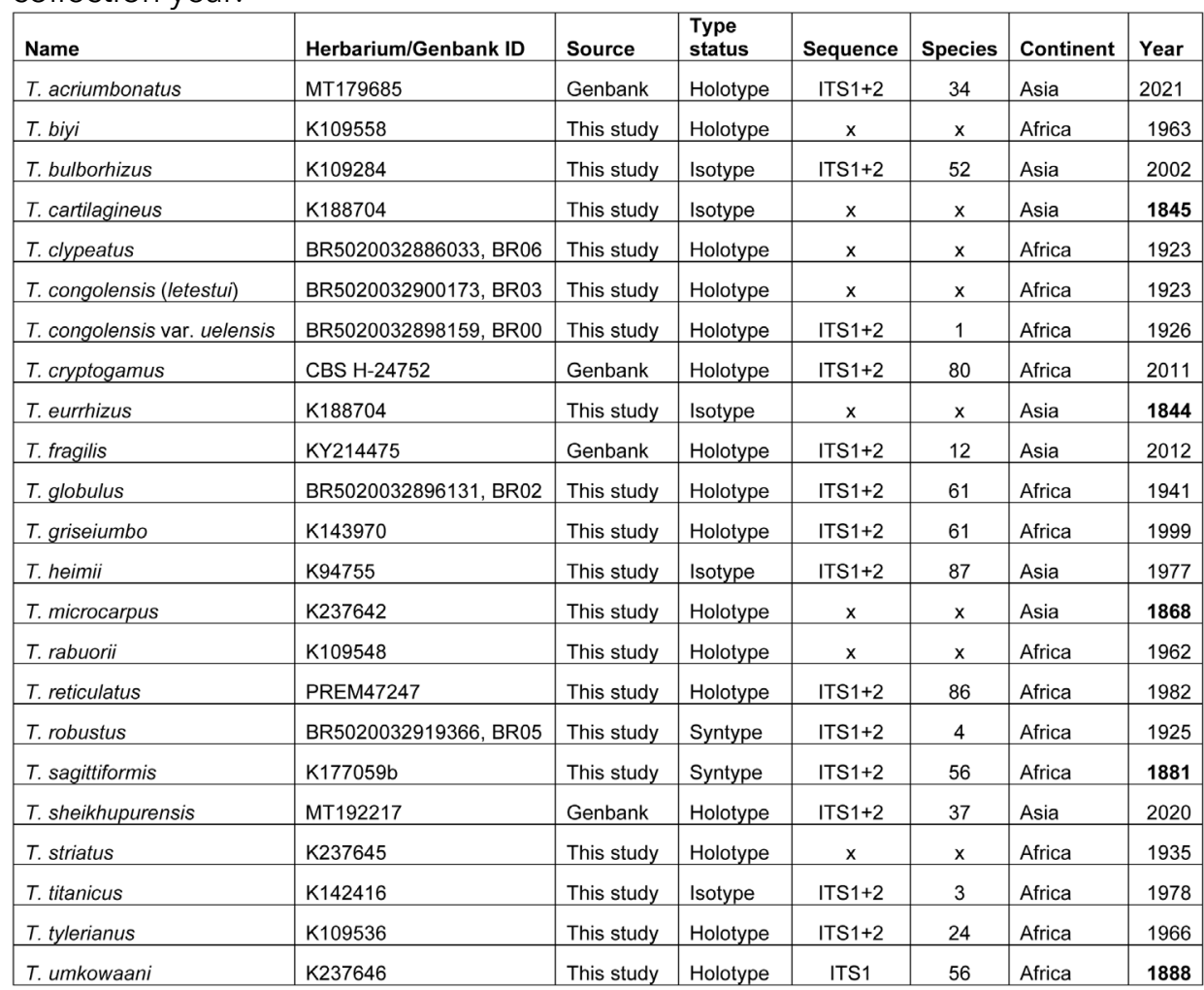

\section{Phylogenetic analysis}

Using a preliminary phylogenetic analysis of the 88 species, we selected 24 taxa that covered the diversity of Termitomyces in our sample for whole-genome sequencing (van de Peppel et al. 2021). The phylogenetic reconstruction shown in Figure 1 used this phylogeny as a constraint. We recovered five main groups (Figure 1). Group 1 contains the symbionts of three genera: Acanthotermes, Pseudacanthotermes and Ancistrotermes. Group 2 contains predominantly the symbionts of Microtermes but also those of Ancistrotermes, and Allodontermes. Group 3 contains the symbionts of Macrotermes. Group 4 is a small group with just two species hypotheses 
and is associated predominantly with Microtermes and Ancistrotermes but also Synacanthotermes. Group 5 is the largest group including more than half of the total number of species hypotheses and containing predominantly symbionts of the genus Odontotermes but also the small genus Protermes. Within this group we can distinguish four sub groups.

We find several examples of incongruence between morphological species concepts and the DNA-based species hypotheses. The three most notable examples are T. clypeatus, for which we could match specimens to five different species hypotheses (species 6, 43, 49, 79) in two major groups (group 1 and 5.3), T. striatus which we also could match to five species hypotheses (species 12, 15, $41,49,72$ ) in four major groups (group 1, 2, 3, 5.3) and T. fuliginosus which matched to four species hypotheses (species 44, 53, 65, 82) in three major groups (group 1, 4, 5.1). T. microcarpus shows a different pattern: although it contains seven species hypotheses, these all belong to group 5.4 . 


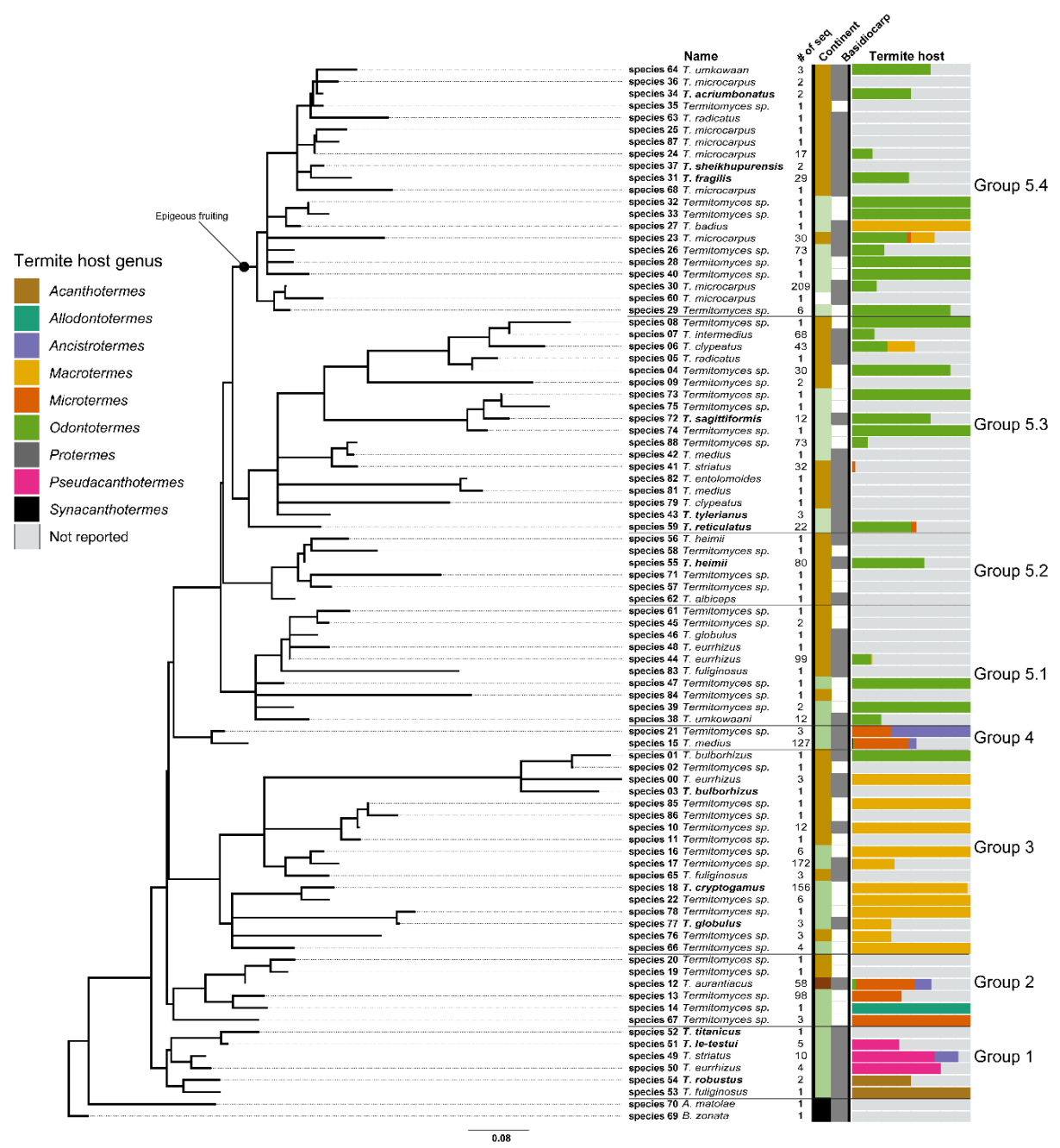

Figure 1 | Phylogenetic reconstruction of representative ITS sequences for each species hypothesis. Names in bold are identifications confirmed by sequences obtained from type specimens. Numbers next to species names indicate the number of sequences that are clustered in a species hypothesis. Different continents are indicated in the third column; Africa (light green) and Asia (brown). Black indicates the American origin of the two outgroups. Whether a species hypothesis could be linked to a sequence obtained from a basidiocarp is indicated in the fourth column; basidiocarp present (dark grey) or absent/not reported (white). The termite host genus for each species hypothesis is indicated in the bar plots on the far right, each bar shows the frequency of the reported host of the total number of sequences. 


\section{Discussion}

\section{Host specificity}

Previous phylogenetic studies on the genus Termitomyces recovered either four or five main symbiont clades and showed moderate host specificity between termite hosts and their symbionts, mainly at the level of these clades (Aanen et al. 2002, Aanen et al. 2007, Nobre, Kone, et al. 2011, van de Peppel and Aanen 2020). The relationships between these five clades were not resolved by previous studies but a recent phylogenomic study managed to resolve these deeper relationships (van de Peppel et al. 2021)(Figure 2). Interestingly this study confirmed the paraphyly of the symbionts of Ancistrotermes (and Microtermes) termites.

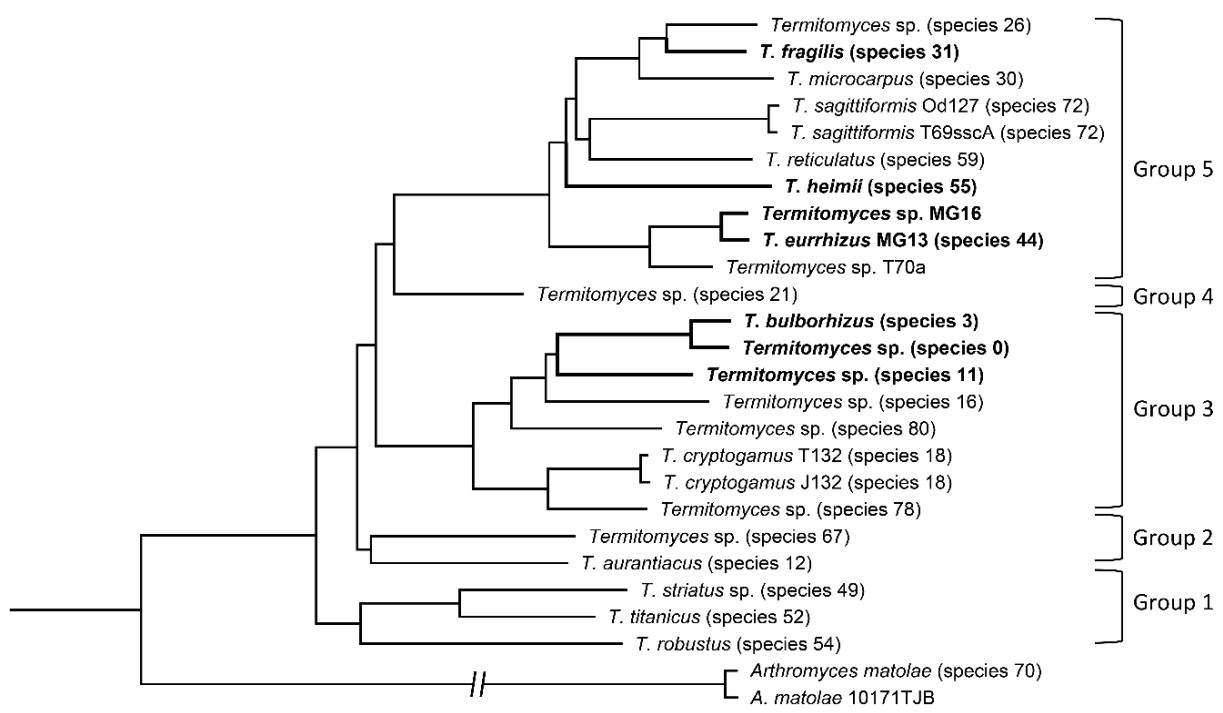

Figure 2 | Termitomyces phylogeny adapted from van de Peppel et al. 2021. showcasing the five major groups within Termitomyces. Names in bold indicate Asian taxa. All branches were significantly supported by either 100 or 99 bootstraps.

In agreement with previous studies we find that each clade has one or two dominant host genera (Figure 1). The paraphyly of the Microtermes and Ancistrotermes symbionts raises the question whether these termites have obtained a new symbiont lineage or whether the termite genera are also paraphyletic. In a subsequent analysis on the wholemitochondrial genome sequences of all fungus-growing termites currently in Genbank (September, 2020) we found some indication that this may be the case as two unidentified Microtermes species cluster 
outside the main clade (Figure 3). Further study is needed to confirm this as there is no biological data on which Termitomyces symbionts are associated with these two termite species. Additionally the genus Ancistrotermes is only represented by a single sequence in this analysis, and this sample is very close to a Microtermes sequence, raising some doubt on this result.

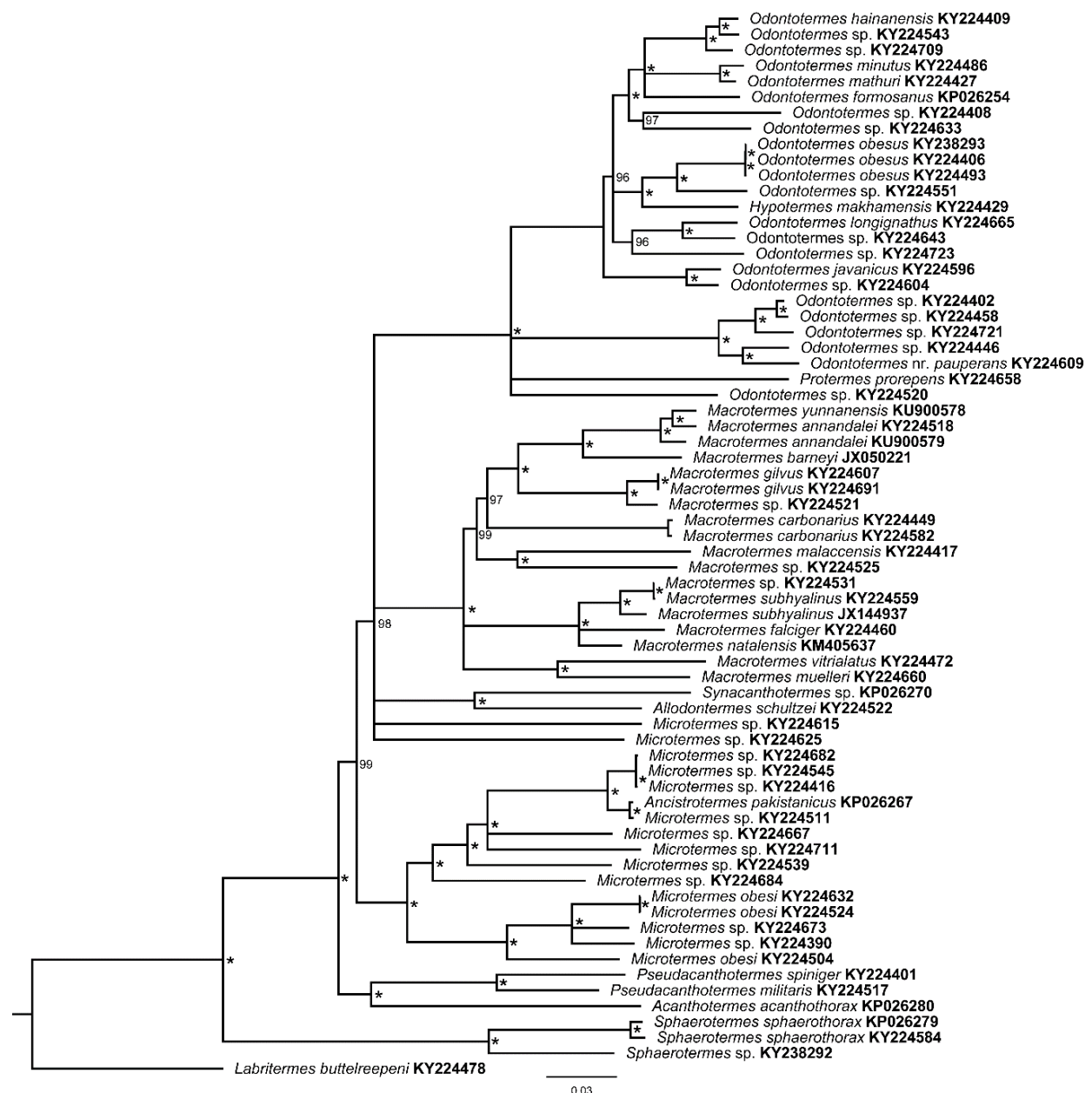

Figure 3 | Maximum-likelihood tree of fungus-growing termites based on the mitochondrial genomes. Numbers at the nodes indicate ultrafast bootstraps, an asterisk indicates 100 ultrafast bootstrap support, nodes with a support value lower than 95 were collapsed into polytomies. Labritermes buttelreepeni was used as an outgroup.

Symbiont sharing appears to be rare between the major genera Macrotermes, Microtermes and Odontotermes on the African continent. In two cases, an Odontotermes symbiont is shared with Microtermes 
termites (species 75 and 86 ) and in two cases it is the other way around (species 27 and 78). Although a rare case of symbiont exchange between these genera seems possible, one should be cautious as these observations based on a single collection could be potential misidentifications. The morphology or architecture of the mound on which a fruiting body is collected is not a reliable identifier for the host genus, as colonies of different genera often co-inhabit a single mound. Co-habitation of Microtermes in the mounds of Ma. natalensis and various Odontotermes spp. occurs frequently (Uys 2002, van de Peppel and Aanen 2020). There are several reports of smaller species of termites who construct their fungus gardens at the base of the large above-ground mounds of Macrotermes species, such as An. latinotus, Odontotermes sp., P. militaris, Allodontotermes sp. and Microtermes sp. (Malaisse 1978, Mujinya et al. 2014). A Termitomyces basidiocarp collected on a termite mound should always be traced back via the pseudorhiza to the host fungus garden where a termite should be sampled for a reliable identification of the termite host (preferably both morphologically and molecularly). The relationship can be confirmed if the fungal sequence from the gut of the worker termites matches the fruiting body sequence (Aanen et al. 2002). Reliable identifications are thus crucial for solid claims on host-specificity.

On the Asian continent there are more reports of Termitomyces species shared among different genera. These species include: $T$. bulborhizus, T. clypeatus, T. eurhizus and T. microcarpus (Pegler and Vanhaecke 1994, Wei, Tang, and Yao 2009). We also find three Asian taxa associated with the three major termite genera: species 23 ( $T$. microcarpus), species 6 (T. clypeatus) and species 44 ( $T$. eurhizus).

We do not have an explanation for this difference in host-specificity between the continents, but independent migrations by host and symbiont may have affected this. For example, if a termite species migrates from Africa to Asia but its symbionts do not, its survival may depend on associating with an aspecific symbiont from a different species or genus.

\section{Geographic separation}

Our analysis generally shows clear genetic divergence between African and Asian taxa as all of the species hypotheses occur either on Africa or on Asia, except for species 12, consistent with a recent intercontinental migration. The divergence between African and Asian taxa is consistent with previous research (Froslev et al. 2003). Even though the symbiosis originated in Africa and only four of the major fungus-growing termite genera occur in Asia, we see no large differences in diversity of 
Termitomyces symbionts between the continents. The Asian species hypotheses make up just over half of the total diversity, 48 out of the 87 species hypotheses (including the one mixed species hypothesis).

Our phylogenetic analysis shows at least seven independent intercontinental migrations in the genus Termitomyces, presumably from Africa to Asia, but back-migrations are also possible as our tree is not completely resolved. The migratory events occur in all five major groups, except for group 1, which is only represented by African species. We identified at least two additional migratory events to the five intercontinental migrations in Termitomyces already documented (Aanen et al. 2002) and four out-of-Africa migrations of the termites (Aanen and Eggleton 2005). The lack of mixed populations within species suggests that migratory events are not very common.

\section{Symbiont transmission mode}

Uniparental vertical symbiont transmission evolved twice independently in the fungus-growing termites, via the female reproductives in the genus Microtermes and via the male reproductives in the species Ma. bellicosus (Johnson 1981, Korb and Aanen 2003, Nobre, Fernandes, et al. 2011). Although uniparental vertical symbiont transmission could increase host-specificity, previous studies did not find evidence for strong host-specificity in both the genus Microtermes and the species Ma. bellicosus (Nobre, Fernandes, et al. 2011, van de Peppel and Aanen 2020). Our analysis is congruent with these previous findings as we found two species (species 16 and 18) from two different sub clades to be associated with Ma. bellicosus. Both these species are also shared with several other species of Macrotermes. The symbionts of the genus Microtermes are also found in two main groups (group 2 and 4) and are also shared with the termite genera Ancistrotermes, Synacanthotermes and in rare cases Odontotermes. That these particular symbionts are shared between different hosts, including those without uniparental vertical transmission, shows that there is little opportunity for co-speciation. Therefore, it seems unlikely that symbiont transmission mode has played a major factor in speciation in the genus Termitomyces.

\section{Host control over fruiting}

We found that 37 of the 87 species hypotheses could not be associated with a sequence from a mushroom. About half of the African species (20 out of 38 species hypotheses) and approximately a third of the Asian species (17 out of 47 species hypotheses) could not be linked to a sequence obtained from a mushroom. Most species of Termitomyces 
produce mushrooms seasonally, usually correlated with the rainy seasons, and with alate production by the termites (Koné et al. 2011, Koné et al. 2018). Some species, especially the symbionts of the termite genus Microtermes and some African species of Macrotermes, seem to produce mushrooms infrequently or not at all (Johnson 1981, Wood and Thomas 1989, Darlington 1994, Korb and Aanen 2003, Nobre, Fernandes, et al. 2011, van de Peppel and Aanen 2020). At least four species of Microtermes occur in South Africa associated with at least four species of Termitomyces (species 12, 13, 15, 67)(Aanen et al. 2007, van de Peppel and Aanen 2020), yet none of the mushroom-forming species of Termitomyces known to occur in South Africa are associated with Microtermes (Van der Westhuizen and Eicker 1990). This is also supported by our study on the herbarium collections of the PREM and PRUM herbaria in South Africa, none of the 92 specimens that we investigated, with collection dates ranging from 1912 to 2008, had Microtermes as the reported host or had a DNA match to one of the four Microtermes symbiont species. Nevertheless, for the absence of clonality among symbiont populations, indicates that sexual reproduction and thus mushroom formation should occur regularly (van de Peppel and Aanen 2020). Of the four South African Microtermes symbiont species, species 12 and 15 are shared with other termite genera with horizontal symbiont transmission such as Ancistrotermes and Synacanthotermes. All mushrooms associated with species hypothesis $15, T$. medius, were associated with termites of the genus Ancistrotermes, and never with Microtermes, which indicates the capacity to form mushrooms is still present, but can be suppressed by the termite host. A similar pattern has been found for populations of the symbionts of a different species with vertical symbiont transmission, Ma. bellicosus, which are predominantly clonal when associated with Ma. bellicosus, but recombining when associated with other species of Macrotermes (Nobre, Fernandes, et al. 2011). A potential case of suppressed fruiting in a Macrotermes species with horizontal transmission is $M$. natalensis and its symbiont: 'T. cryptogamus'. No natural mushrooms have been reported for this species, but under laboratory conditions in the absence of the termite host it can form mushrooms which produce viable spores (De Fine Licht, Andersen, and Aanen 2005, Vreeburg et al. 2020). So it seems that differences in the frequency of mushrooms formation between groups can be explained by suppression of mushroom formation by some termite species but not by others (Korb and Aanen 2003). Further experimental studies are needed to confirm this hypothesis. 

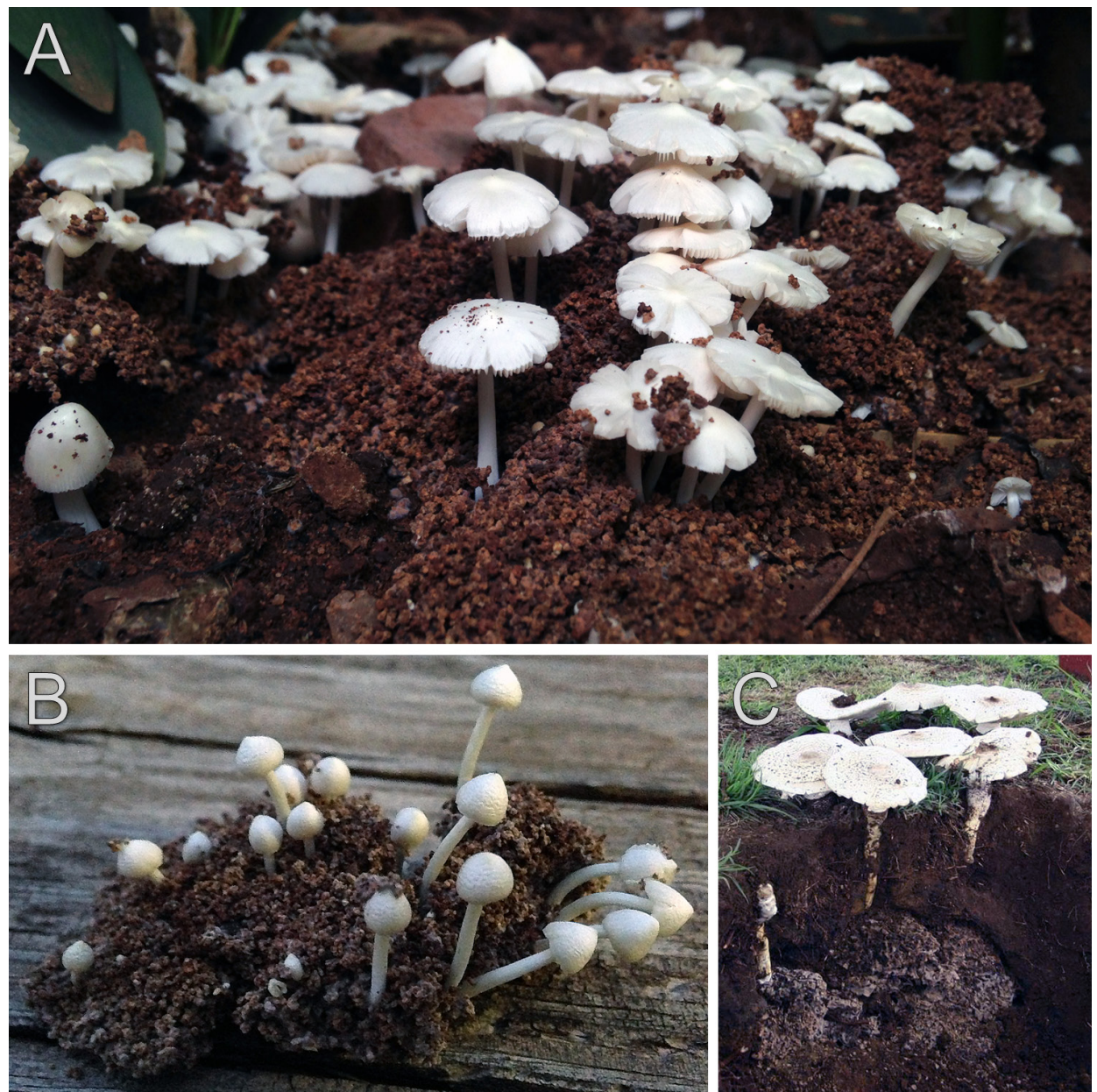

Figure 4 | Photos depicting the differences between the two fruiting modes in Termitomyces. A: Epigeous fruiting of African T. microcarpus (species 30) showing basidiocarps produced on ejected comb material. B: No connection between comb and basidiocarp is present. C: An example of the more common fruiting mode; hypogeous fruiting, showing the attachment of $T$. reticulatus (species 59) to a fungus comb of Odontotermes sp.

\section{The evolution of epigeous fruiting}

With a cap diameter of less than two centimetres, $T$. microcarpus is the smallest species of Termitomyces. It grows in large patches of up to hundreds of mushrooms. It differs from other species of Termitomyces in that it fruits on comb material that termites eject from the nest (Figure 4). Unlike all other species of Termitomyces it does not produce a pseudorhiza, although a tiny pseudorhiza has been reported (Horak 1968). The atypical fruiting behaviour and the lack of a pseudorhiza made Heim (1977) think that this way of fruiting closely resembled the 
ancestral state and he therefore placed T. microcarpus in its own subgenus: Prae-Termitomyces. Molecular studies show that the opposite is true, as T. microcarpus is nested within Termitomyces so that this kind of mushroom formation is derived (Aanen et al. 2002, Froslev et al. 2003).

It might seem likely that $T$. microcarpus has a limited host range because of this unique fruiting behaviour, if it would exploit a specific termite behavioural activity. However, this does not seem to be the case, as in Africa this species is associated with 0 . badius, $O$. transvaalensis and O. vulgaris (Bottomley and Fuller 1921, Sands 1969, Van der Westhuizen and Eicker 1990) but also with two species of the genus Protermes; P. minutus and P. prorepens (Aanen et al. 2002). In Asia it is associated with $O$. malaccensis, $O$. redemanni but also species in the genus Ancistrotermes (Pegler and Vanhaecke 1994), Hypotermes xenotermitis (Aanen et al. 2002) and Ma. barneyi (Wei, Tang, and Yao 2009). Here, we found additional host species, in Asia: Ma. gilvus, Ma. annandalei, Microtermes sp., O. longignathus, and O. formosanus, and in Africa: Mi. subhyalinus. Importantly, most of these species are also known to associate with different Termitomyces symbionts. For example, $O$. badius is known to associate with $T$. umkowaani and $T$. reticulatus and $O$. transvaalensis with $T$. reticulatus (Van der Westhuizen and Eicker 1990). Surprisingly, this broad host range implies that this typical behaviour of ejecting comb material by the termites is induced by the fungus, as termites perform this behaviour only when associated with $T$. microcarpus. This is in sharp contrast with what we described in the previous paragraph in the termite genus Microtermes, where the termite seems to have some level of control over fruiting of the symbiont.

Our phylogenetic reconstruction shows that all samples identified as T. microcarpus are placed within one clade (group 5.4) indicating that epigeous fruiting evolved once. Since the Asian taxa form a sub-clade within the main clade, epigeous fruiting most likely evolved on the African continent. Group 5.4 consists of 21 species hypotheses and includes several species names, showing that the name T. microcarpus is paraphyletic and does not represent a single species but rather a group of species exhibiting a similar phenotype. This causes some taxonomical issues, as the type specimen has been described from a collection from Sri Lanka (Berkeley and Broome 1871). Despite repeated attempts, we did not manage to obtain a sequence from the type specimen of $T$. microcarpus and therefore we cannot accurately assign the name to a species hypothesis. Species 36 represents a T. microcarpus collection from Sri Lanka, the country of 
origin from the type collection, which could represent the same species as the type collection. This means that the African T. microcarpus collections (species 30) represents a different species and should therefore be renamed, which may not be warranted as it is a very common species in Africa (Pegler 1977, Van der Westhuizen and Eicker 1990). This is also reflected by the 209 sequences which make up species 30 in our analysis. Other species with either epigeous fruiting or indications for epigeous fruiting (lack of a pseudorhiza) include: $T$. badius, T. indicus, T. narobiensis, and T. orientalis (Otieno 1964, 1968, Natarajan 1975), although some authors conclude that these species all are synonyms for T. microcarpus (Pegler 1977, Pegler and Vanhaecke 1994, Wei, Tang, and Yao 2009, Osiemo et al. 2010). Our single specimen identified as T. badius (species 27 ) is genetically distinct from specimens identified as T. microcarpus, this may indicate that $T$. badius could be a different species. We were unable to obtain specimens for $T$. indicus, $T$. narobiensis and $T$. orientalis, so their relationship to $T$. microcarpus remains unclear.

A 'semi-hypogeous' form of T. microcarpus has been reported of which the stipe can be up to eight centimetres belowground and multiple fruiting bodies can be connected (Heim 1942, 1977). Heim also notes that there is no connection between the basidiocarps and the fungus comb. We were able to match an unidentified fruiting body (VDWALT1577) to this description in species hypothesis 26 (R. van der Walt, pers. comm.). It is therefore likely that this semi-hypogeous form represents a different species, although it belongs to clade 5.4. Other species from group 5.4 which produce a pseudorhiza and therefore may represent semi-hypogeous fruiters are $T$. acriumbonatus, $T$. fragilis, T. radicatus and T. sheikhupurensis (Natarajan 1977, Ye et al. 2019, Usman and Khalid 2020, Izhar, Khalid, and Bashir 2020). Growth on ejected comb material is reported for T. sheikhupurensis (Izhar, Khalid, and Bashir 2020) and for T. fragilis a vague connection to a fungus garden has been reported (Ye et al. 2019). The phylogenetic position of $T$. radicatus remains ambiguous as two species hypotheses (species 5 and 63) with sequences of $T$. radicatus appear in two different groups (group 5.3 and 5.4).

We found no clear evidence for a reversal from fruiting on ejected comb material to fruiting directly from the fungus comb in group 5.4. Only in some species this ejected comb material may reach the soil surface and produce the typical habit lacking a pseudorhiza. In other cases, the comb material may be expelled from the nest but remain buried in the soil and therefore a (short) pseudorhiza is produced. The mechanism by which the fungus causes the termites to 
eject comb material from their nest is an interesting topic for future study.

\section{Taxonomical challenges}

Morphology-based taxonomy has greatly hampered the identification and description of new species of Termitomyces, as basidiocarps are seasonal and not frequent in many species. The symbionts of several species of Microtermes (Wood and Thomas 1989, Darlington 1994, van de Peppel and Aanen 2020) as well as several species of Macrotermes (de Fine Licht, Boomsma, and Aanen 2006, Koné et al. 2011) even have no reports of basidiocarps. The rarity of basidiocarps in a substantial number of species begs for a different approach to the taxonomy of Termitomyces. Although differences in morphology occur in laboratory cultures, these differences are not sufficient and reliable enough to distinguish between species (Botha and Eicker 1991a, b, Tibuhwa 2012). An approach to taxonomy using well-characterized and publicly accessible laboratory cultures in combination with molecular data has been suggested (Makonde et al. 2013). Thus far, only a single species, T. cryptogamus, has been described using this approach (van de Peppel et al. 2021 under review).

This study shows that there generally is a clear genetic differentiation between African and Asian taxa of Termitomyces, indicating substantial geographical isolation between continents. Although certain species names, such as T. clypeatus, T. eurhizus and $T$. microcarpus are used for the same morphospecies, African and Asian specimens of these morphospecies are clearly genetically divergent. This may cause problems in scientific communication as specimens from different continents identified under the same name generally represent different phylogeneticspecies and possibly also separate biological species. This is clearly the case for T. microcarpus, where the African and Asian are not part of the same species hypothesis and cluster in separate sub-clades within clade 5.4. Also different species hypotheses within continents bear the name T. microcarpus, indicating additional cryptic diversity. Two species with a very similar morphology, T. umkowaani and T. eurhizus, have been synonymized by some authors (Van der Westhuizen and Eicker 1990, Pegler and Vanhaecke 1994). This similar or identical morphology can be explained by shared ancestry as we find that T. umkowaani (species 38) and T. eurhizus (species 44, 48) are part of two distinct sub-clades in group 5.1. In other cases, morphological similarity is not due to shared ancestry, but due to non-homologous processes, for example convergent evolution. This is 
the case for $T$. striatus and $T$. clypeatus, as specimens of these species are found scattered in the phylogenetic tree.

Previous examples indicate that a species concept based on morphological diagnostic features of the mushroom is not congruent with our phylogenetic species concept. A morphological species concept does not seem useful in the genus Termitomyces as it is very inconvenient to describe common species with rare fructification events and because there appears to be a high degree of cryptic species diversity. We therefore suggest delineating species primarily based on d DNA-sequence similarity, preferably in combination with additional features such as termite host genus, geographic origin, and fruiting mode. Additionally, to understand the significance of DNA divergence for reproductive isolation, the application of a biological species concept will also be highly valuable.

\section{Conclusion}

In this study, we delineated species using an automated barcode gap discovery approach on a large dataset of ITS sequences. Using this method, we identified 87 species of Termitomyces. We identified several factors that may have contributed to the current diversity such as host-specificity, geographic separation, host control over fruiting and fruiting mode. We conclude that uniparental vertical symbiont transmission probably has only a minor contribution to diversification in Termitomyces. There are probably several other factors which may have caused diversification but which were not part of this study, such as substrate preference of changes in host diet (da Costa et al. 2019). This study helped to improve our understanding of several processes which may have shaped current diversity in Termitomyces but further study is needed to confirm some of the hypotheses that we formulated. 


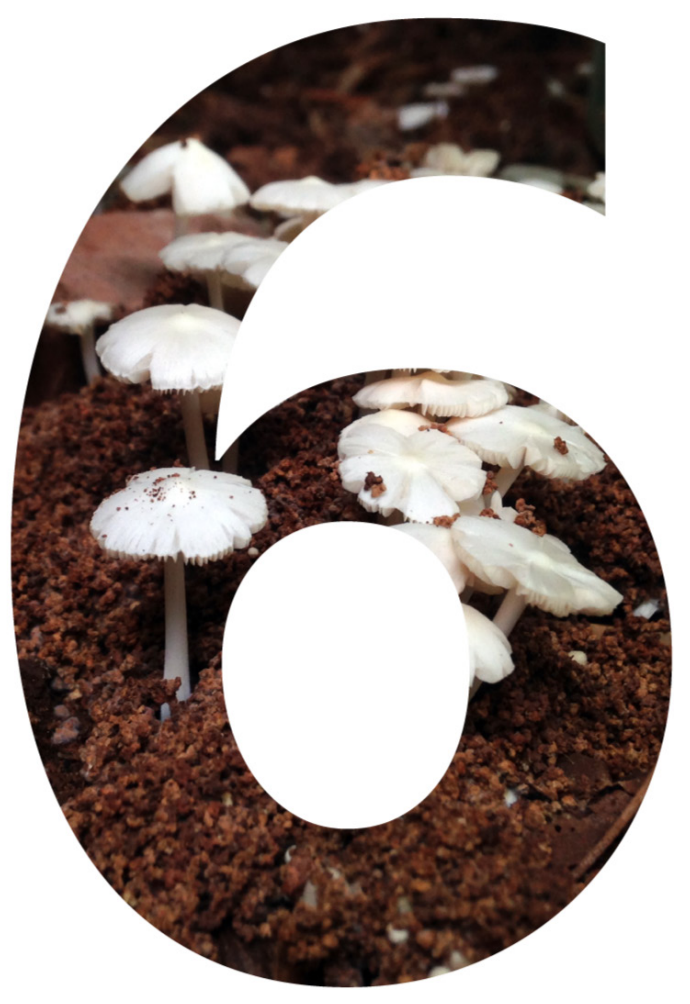

High diversity and low host-specificity of Termitomyces symbionts cultivated by Microtermes spp. indicate frequent symbiont exchange

Lennart J.J. van de Peppel Duur K. Aanen

Published in Fungal Ecology 


\section{Abstract}

Fungus-growing termites (subfamily Macrotermitinae) live in an obligate mutualistic symbiosis with species of the fungal genus Termitomyces (Basidiomycota). Although the species that build large mounds are the most conspicuous, termites of the genus Microtermes construct large underground networks of tunnels connecting many fungus gardens. They are also the only entire genus within the Macrotermitinae in which vertical transmission of the fungal symbiont has evolved. To study patterns of genetic diversity in species of the genus Microtermes and their Termitomyces symbionts, we sampled at three different locations in South Africa and sequenced $\mathrm{COI}$ for the termites and ITS for the fungi. We discovered high genetic diversity in both termites and fungal symbionts but very low interaction specificity. This implies that frequent horizontal exchange of fungal symbionts occurs between species, despite vertical transmission across generations. We also estimated colony size based on termite haplotype and fungal genotype combinations and found indications that colonies may extend over large areas. 


\section{Introduction}

Termites (order Isoptera) are a large and diverse insect group. They play an important role in ecosystem functioning as decomposers, especially in the tropics (Jouquet et al. 2011). In one extant group of termites, the family Termitidae (also called 'higher termites'), several highly specialised feeding types have evolved, such as soil feeding (Inward, Vogler, and Eggleton 2007). Within the Termitidae, all species of the subfamily Macrotermitinae have evolved a unique feeding habit; they live in an obligate symbiosis with species of the basidiomycete fungus Termitomyces (Johnson et al. 1981, Wood and Thomas 1989). The origin of the symbiosis has been estimated at 30 million years ago in the rain forests of Africa (Aanen and Eggleton 2005, Roberts et al. 2016). The fungus-growing termites have a single origin and their symbiotic fungus Termitomyces (Basidiomycota, Lyophyllaceae) as well, so that there has been a single domestication only (Aanen et al. 2002). The distribution of fungus-growing termites ranges from sub-Saharan Africa, including Madagascar, to Asia (Wood and Thomas 1989, Nobre, Eggleton, and Aanen 2010). Currently there are 10 described genera in the Macrotermitinae of which six occur in southern Africa (Eggleton 2000, Uys 2002). The most conspicuous fungus-growing termites construct large epigeal mounds but other genera have subterranean colonies in which they farm their fungus. The termites grow their fungus on a special substrate called the fungus comb, which they build from primary feces consisting of plant material inoculated with asexual spores. They harvest the asexual fruit bodies which serve as a proteinrich food source, a source of additional digestive enzymes, inoculum for new fungus combs and a source of the essential amino acid tryptophan (Martin and Martin 1978, Nobre and Aanen 2012, Leuthold, Badertscher, and Imboden 1989, Chiu et al. 2019).

Previous studies have shown that there is some host specificity between termite and fungus at the generic level but not at the species level (Aanen et al. 2002, Aanen et al. 2007, Osiemo et al. 2010). One of the factors influencing host-specificity is symbiont transmission. In the Macrotermitinae horizontal transmission is the main transmission mode; basidiospores produced by the sexual fruit bodies (mushrooms) are used to inoculate the fungus gardens of newly founded colonies (Johnson et al. 1981, Korb and Aanen 2003, de Fine Licht, Boomsma, and Aanen 2006). However, in the genus Microtermes and in a single species of the genus Macrotermes, M. bellicosus, vertical transmission has evolved. In both cases vertical transmission is uniparental, but in opposite ways: in Microtermes the female reproductives, and in $M$. bellicosus the male reproductives, carry asexual fungal spores in the gut 
to inoculate a new fungus garden (Johnson 1981, Johnson et al. 1981, Nobre, Fernandes, et al. 2011). Theory predicts that vertical transmission should reduce host-symbiont conflicts and increase hostspecificity (Frank 1996b). Consistent with vertical symbiont transmission, fruit bodies have never been observed for Microtermes (Johnson et al. 1981, Darlington 1994). If transmission is strictly vertical, the fungus will only be clonally propagated, and in the long term genetic variation in Microtermes symbionts will be reduced.

However, previous studies on genetic variation of Microtermes symbionts have given conflicting results (Aanen et al., 2002; Aanen et al., 2007). Microtermes species from western Africa all share one single genotype of a single species of Termitomyces and this genotype was also found in species of the genera Ancistrotermes and Synacanthotermes (Aanen et al. 2002). In contrast, in South Africa a big pool of genetically diverse symbionts was associated with species of the genus Microtermes (Aanen et al. 2002, Aanen et al. 2007). However, sampling in these studies has been limited and only on large spatial scales so it remains to be studied how these different patterns can be explained.

In the present study we have extensively sampled fungus gardens of species of the genus Microtermes on smaller spatial scales in South Africa to study the population genetics of Termitomyces and how variation is distributed between species and colonies. We sampled at three different field sites and collected sequence data for both termite host and fungal symbiont. By studying genetic diversity of both host and symbiont we estimated the number of different colonies inhabiting the mounds of $M$. natalensis or Odontotermes spp. We also estimated the average size of a colony and the potential spatial area of a colony. Furthermore we looked for any patterns of host-specificity and whether horizontal transmission of the symbiont occurs.

\section{Material and Methods}

Fungus-growing termites of the genus Microtermes have a very inconspicuous lifestyle; for example, they do not construct large epigeal mounds as species of some other genera such as Macrotermes do, but instead make large subterranean networks of tunnels connecting many different small fungus gardens (Uys 2002). There is a documented case where two foraging $M$. najdensis termites from the same colony have been collected 42 meters apart, indicating that colonies may stretch over long distances (Pearce et al. 1990). Knowledge is lacking on the exact size of colonies (in both horizontal and vertical extent), the number of fungus-gardens per colony, colony density and species 
richness on a local scale. This may be due to sampling bias towards the fungus-growing termite species with conspicuous mounds which are easier to find. Due to the lack of taxonomically informative characteristics it is extremely hard to identify Microtermes termites to the species, which may be another reason why they have been overlooked in many ecological studies (Hausberger et al. 2011). Microtermes often inhabits the mounds of other fungus-growing termites such as Macrotermes natalensis and Odontotermes spp. (Uys 2002)(Aanen, van de Peppel Pers. observations). Because of the high concentration of Microtermes fungus gardens in a relatively small surface area, we chose these mounds as our study sites for collecting Microtermes fungus gardens and termites (Figure 1).

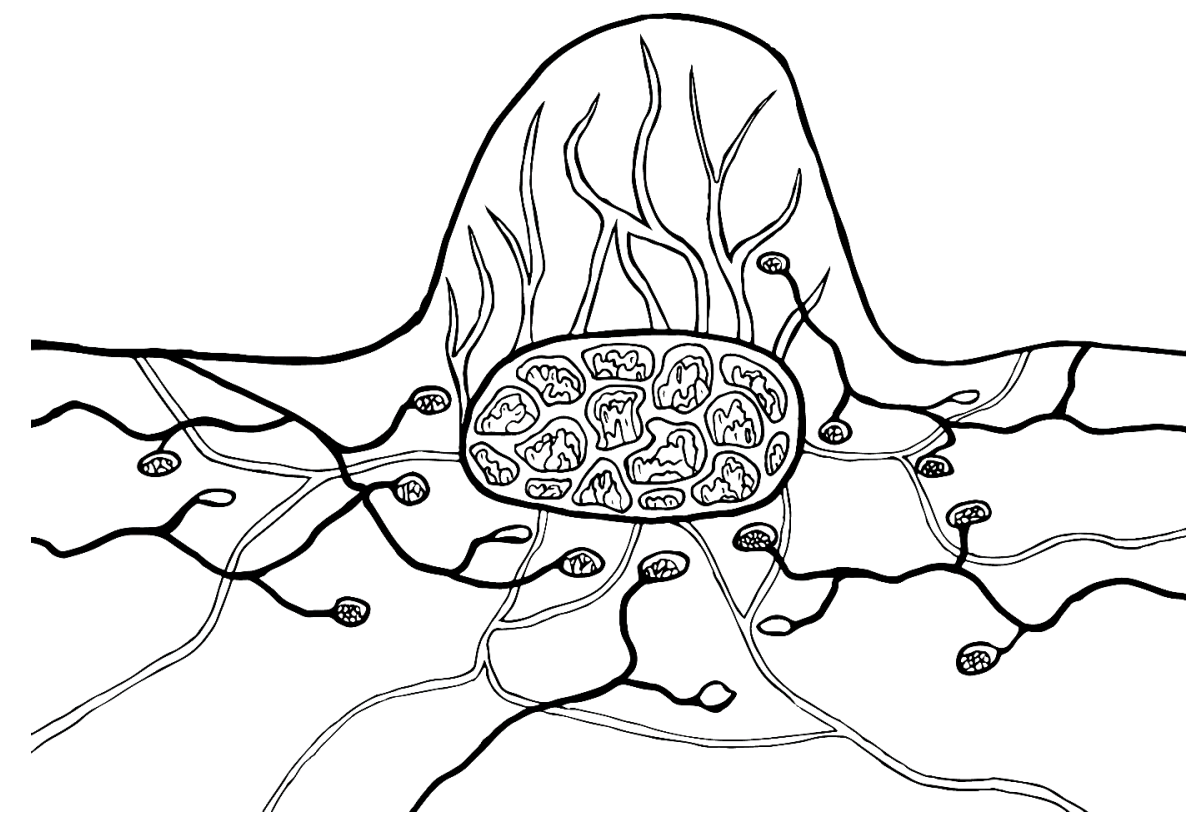

Figure 1 | Schematic overview of the organization of five Microtermes colonies (solid black lines) around a mound of $M$. natalensis.

\section{Sampling locations}

Termites and fungus combs were sampled in South Africa between the $14^{\text {th }}$ and $28^{\text {th }}$ of January 2016 at two different sites in Pretoria [Experimental farm (EF) and Plant Protection Research (ARC)] and at one site in Mookgophong (MO) (Figure 2). Termites and fungus comb were sampled when encountered during the excavation of Macrotermes natalensis and Odontotermes spp. mounds. A total of 11 mounds were sampled; five mounds ( $4 \times \mathrm{M}$. natalensis, $1 \times$ Odontotermes sp.) at $\mathrm{EF}$, three mounds ( $2 \times$ M. natalensis, 1x Odontotermes sp.) at ARC and 
another three at $\mathrm{MO}(3 \times \mathrm{M}$. natalensis). Fungal cultures were made on the collection day or the day after from comb material. Three nodules were placed on a malt yeast extract agar (MYA; per litre demi water: 20 $\mathrm{g}$ malt extract, $2 \mathrm{~g}$ yeast extract, $15 \mathrm{~g}$ agar) plate using sterile forceps. Pieces of each fungus comb were stored in $2 \mathrm{ml}$ tubes with pure ethanol. Since termites from the genus Microtermes are hard to identify on the basis of morphology, termites were only identified at the genus level (Korb et al. 2019). Termites were stored in $1.5 \mathrm{ml}$ Eppendorf tubes with pure ethanol on the collection day. Samples were kept at $4^{\circ} \mathrm{C}$ prior to DNA extraction.

In total 164 fungus comb samples and 134 termite samples were collected. For the final analysis, we only used the collections for which we had both a termite and a fungal symbiont sample.

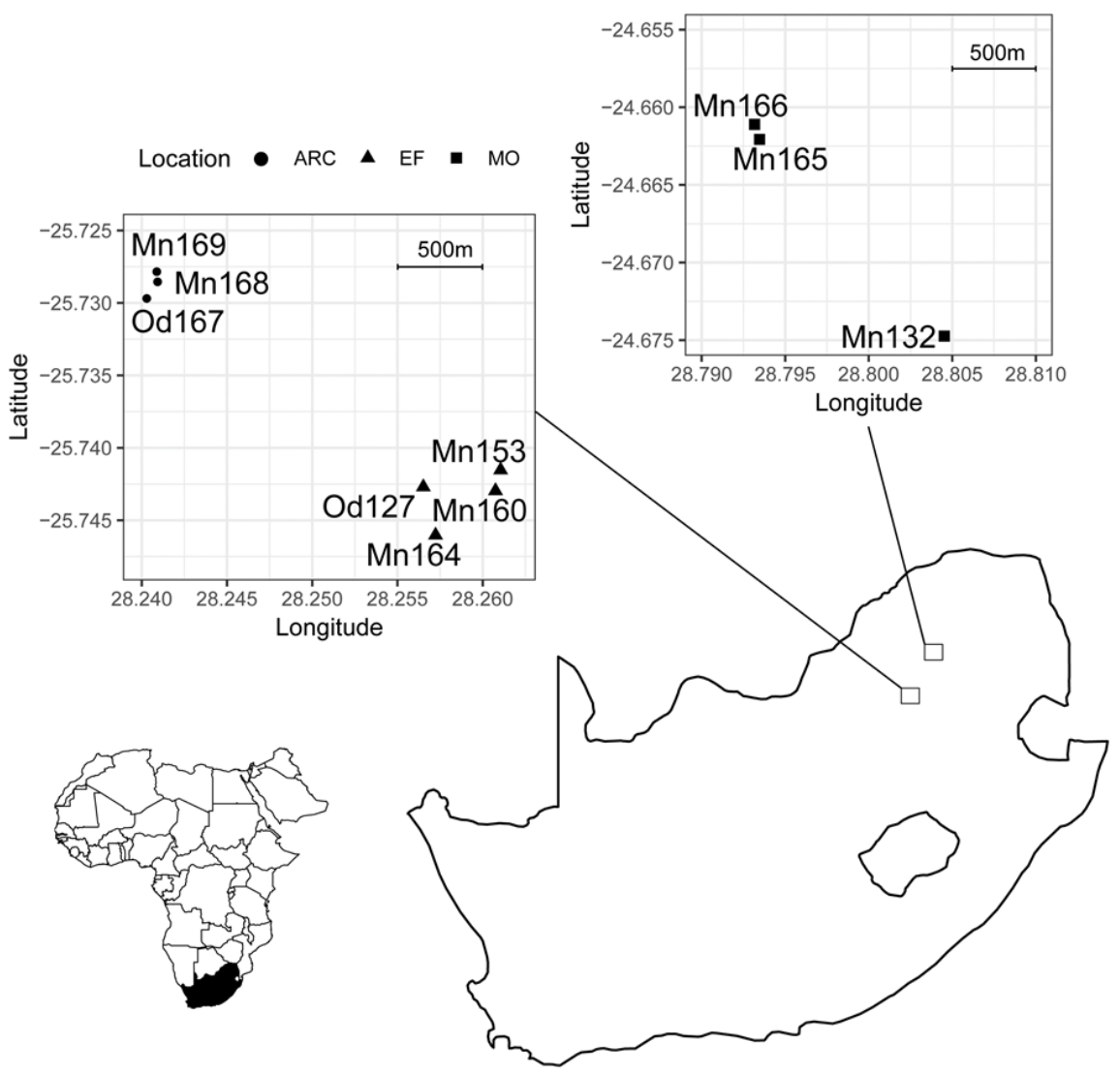

Figure 2 Sampling locations within South Africa [Experimental farm (EF), Plant Protection Research (ARC) and Mookgophong (MO)] and mounds sampled. 


\section{DNA isolation, PCR and sequencing}

For termite DNA isolations the head of a single termite was used. Initial DNA isolations on whole termites did not yield DNA of sufficiently high quality for polymerase chain reactions (PCRs), probably due to minerals and soil particles in the gut. For fungal DNA isolations, a small piece of mycelium $(0.5 \mathrm{gr})$ from a culture was used. All DNA isolations were done following a cetyltrimethylammonium bromide (CTAB) protocol (Nieuwenhuis et al. 2019). Partial sequences of the mitochondrial cytochrome oxidase I (COI) gene were obtained for the termites using a standard PCR reaction using the termite specific primer pair TL1862 and TH2877 (Aanen et al. 2002, Aanen et al. 2007). Sequencing was done using only the forward primer and in some cases using both forward and reverse primer if the forward sequence was too short. For the Termitomyces symbionts the nuclear ribosomal region containing the internal transcribed spacer 1 (ITS1), 5.8S and the internal transcribed spacer 2 (ITS2) was amplified using a standard PCR reaction using the fungal specific primer ITS1f and the general reverse primer ITS4 (White et al. 1990, Gardes and Bruns 1993). Primer sequences, the recipe for the PCR master mix and the PCR programs can be found in Supplementary document 1. Sequencing was done by Eurofins Genomics (Ebersberg, Germany). Forward and reverse sequences were assembled using CLC genomics workbench 8.

\section{Alignments, phylogenetic analysis and haplotype analysis}

Chromatograms were checked in CLC genomics workbench 8. Since fungal heterokaryons contain two genetically different nuclei, they can be heterozygous for the analysed sequences and the chromatograms from fungal sequences were checked for double peaks. These double peaks were assigned with the correct letter according to the standard International Union of Pure and Applied Chemistry (IUPAC) notation for DNA. In the samples for which length polymorphisms occurred full sequences were generated by combining the forward and reverse sequence, in this case always the longest variant of the two variants was used. In two cases two length polymorphisms were found and parts of the sequence were not recovered. The nucleotides that could not be recovered were replaced by question marks in the alignment. Because of these double length polymorphisms, both these sequences were treated as unique fungal genotypes (genotype 1Cx and genotype 1Dx). Additional sequences from previous studies were extracted from Genbank and were added to the alignment (Supplementary Tables 1 and 2). Sequences were aligned using online MAFFT Multiple Sequence Alignment software version 7 using the default settings 
(Katoh and Standley 2013, Katoh, Rozewicki, and Yamada 2017). Bayesian trees were reconstructed for both alignments and the posterior probability (Bayesian) estimates were calculated using Mr. Bayes 3.2.6. (Ronquist et al. 2012) using a general time-reversible model with gamma distribution (GTR+G), 30,000,000 generations and a sampling frequency of 4.000 . The first $25 \%$ of the samples were discarded (burninfrac $=0.25$ ). For each dataset the $50 \%$ majority rule consensus tree was constructed using the post burn-in samples from the posterior distribution of trees. The analyses were run on the CIPRES science gateway (Miller, Pfeiffer, and Schwartz 2010).

Genotype numbers were assigned to each sample corresponding to the main clades that were recovered from the phylogenetic analysis. A letter was added for each unique genotype within those clades if multiple genotypes were present. For further analysis only pairs of termite haplotypes and fungal genotypes were used; all single types were removed from the dataset. Two haplotype/genotype pairs were also excluded from the dataset because they were the only representative of a mound (mounds: Mn168 and $\mathrm{Mn169}$ ). The final dataset used for the analysis consisted of 85 pairs of termite haplotypes and fungal genotypes (Supplementary Table 3). Identical combinations of termite and fungal type at one mound were considered to be from the same Microtermes colony. This is based on the assumption that the termites cultivate their fungal symbiont in a monoculture (Aanen et al. 2002, Katoh et al. 2002, Shinzato et al. 2005). We did not identify the species either for the termites nor for the Termitomyces symbionts, so we will refer to each clade by their haplotype or genotype number.

\section{Results}

\section{Diversity}

A total of 96 termite sequences and 98 fungal sequences were obtained and used for phylogenetic reconstruction. Phylogenetic analysis of the termite samples revealed three small clades and one large clade which contained $83.3 \%$ of all the samples (Figure 3 ). In two of the four clades there was genetic variation within the clade. A similar pattern was found in the fungal tree where four main clades were recovered with the most dominant clade containing $61.2 \%$ of the samples and further genetic variation was present in three of the four clades (Figure 4).

Diversity was highest at location $\mathrm{MO}$ for both termites (Shannon index: 1.82) and fungus (Shannon index: 2.42). Seven of the eight different termite haplotypes and 14 of the 22 fungal genotypes were 
present at this location (Figure 5). At location EF termite diversity was the lowest (Shannon index: 0.39 ) but fungal diversity was relatively high (Shannon index: 1.7). Low diversity for both termites and fungus was found at location ARC (Shannon index: termites 0.5 and fungus 0.5 ). Here only two termite haplotypes and two fungal genotypes were present.

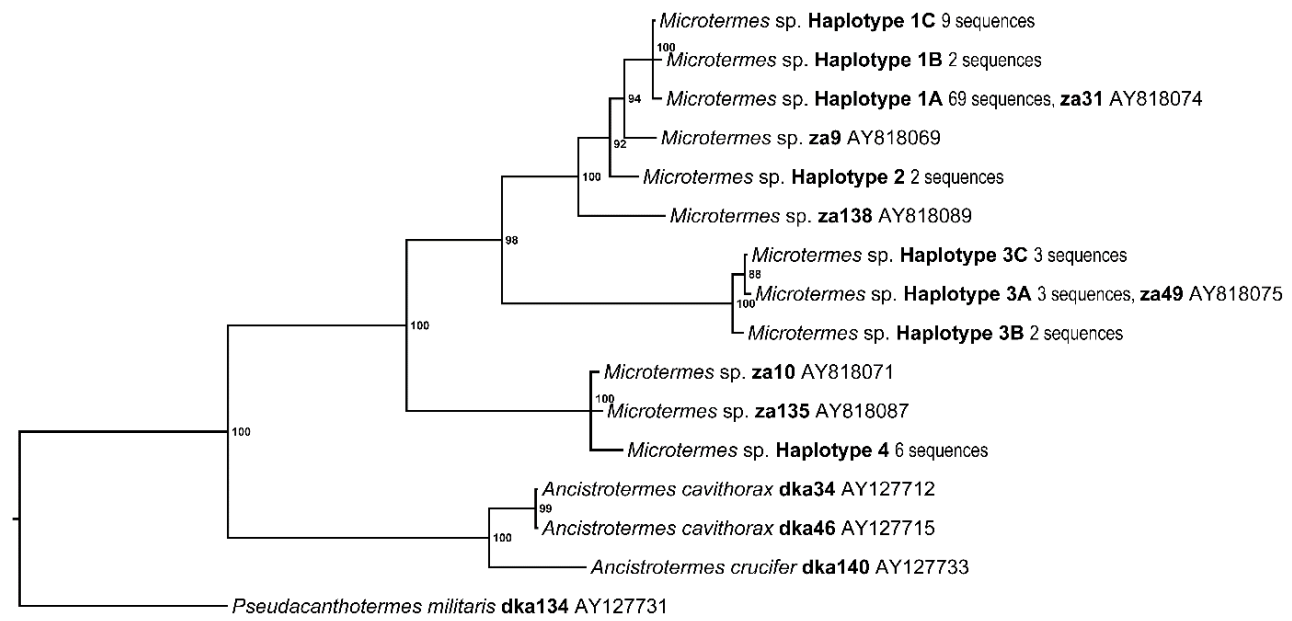

Figure 3 | Majority rule consensus tree of unique termite $\mathrm{COI}$ sequences. The number of identical sequences found in this study for each haplotype is indicated at the tip label. Node labels indicate posterior probabilities.

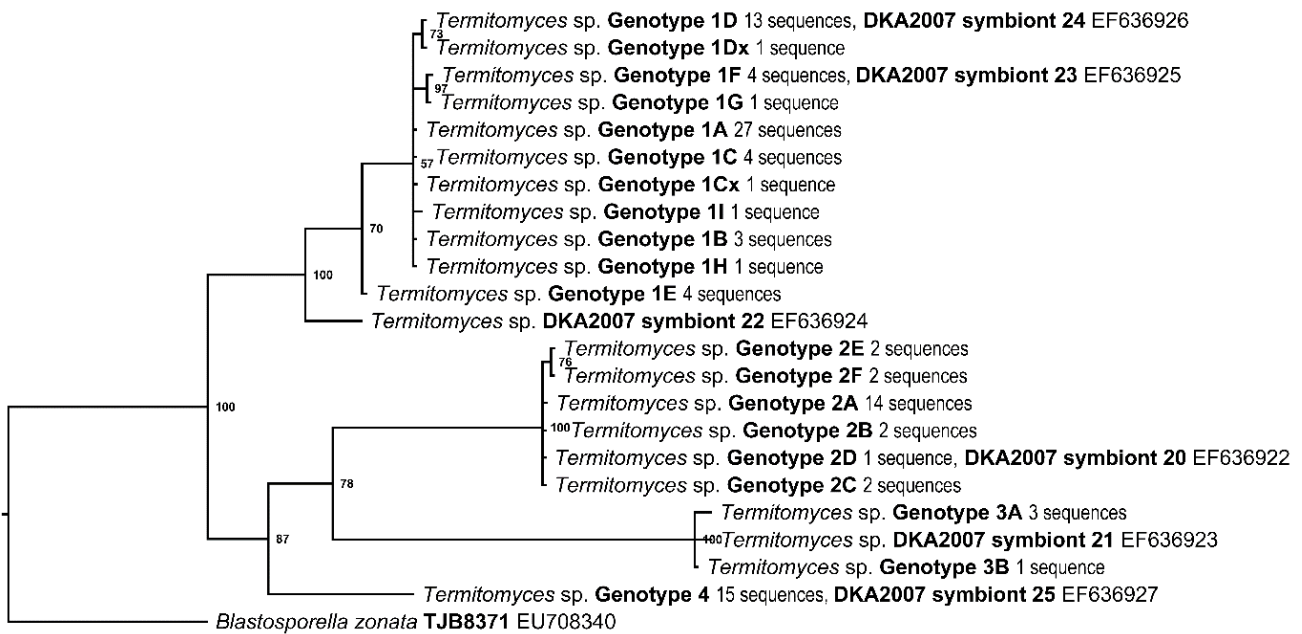

Figure 4 | Majority rule consensus tree of unique Termitomyces ITS sequences. The number of identical sequences found in this study for each genotype is indicated at the tip label. Node labels indicate posterior probabilities 


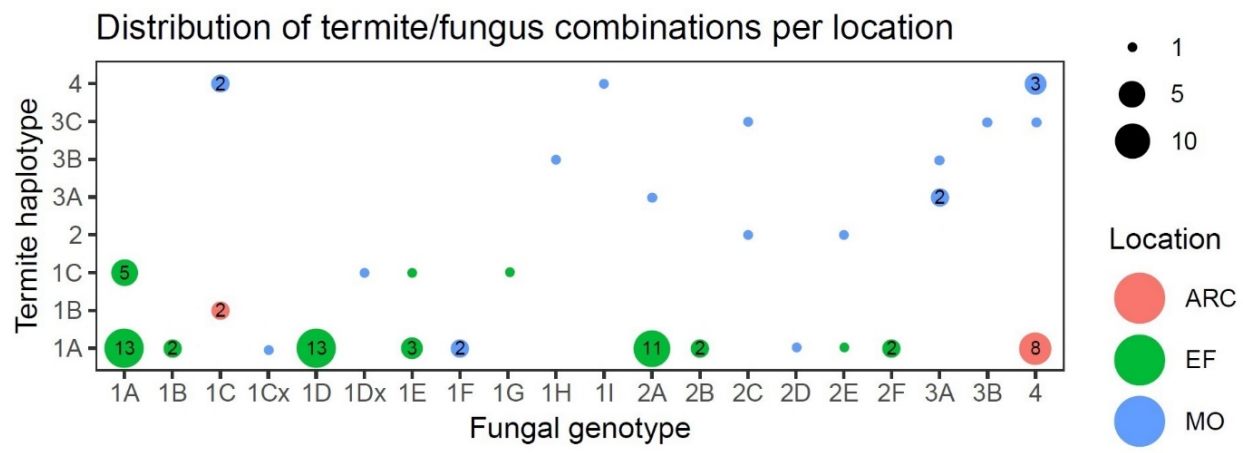

Figure $\mathbf{5}$ | Distribution of termite and fungal haplotypes for each location. The sampling locations are indicated by colour $(A R C=$ red, $E F=g r e e n$ and $\mathrm{MO}=$ blue). The size of the dot indicates the number of samples with the same types that were found.

\section{Colony size estimation and host-specificity patterns}

In total, 87 pairs for which both a fungal sequence and a termite sequence could be obtained were used for this analysis. The number of unique haplotype/genotype combinations found was 29. We considered that multiple counts of one haplotype/genotype combination at one mound were from the same colony. The largest potential colonies were found at mound Od127 and Od167 both consisting of at least eight fungus gardens. Overall the average number of sampled fungus gardens belonging to one colony was 2.36 per mound. Colony diversity was generally very high with on average 4.5 colonies per mound and up to ten different colonies per mound.

\section{Discussion}

\section{Diversity}

In general we found termite and fungal symbiont diversity to be highest at locations EF and MO. The diversity at ARC may in reality be higher, however, because of our limited sampling at this location. The results of this study are consistent with those of previous studies based on more limited sampling. Aanen et al. (2007) found four species of Microtermes and six different $\mathrm{CO} 1$ haplotypes in South Africa. In the present study we found two exact matches to these haplotypes and another six new ones. On the fungal side Aanen et al. (2007) found six different ITS genotypes, of which we found four in the present study. We found another 16 additional fungal genotypes. The discrepancy between host (eight haplotypes) and symbiont (20 genotypes) can partially be explained by the heterokaryotic nature of the fungus. Whereas the 
mitochondrial $\mathrm{COI}$ used for the termites is a haploid marker, we used the nuclear ITS marker for the fungus. Since Termitomyces has two nuclear types and each can have a different ITS sequence, there is more opportunity for unique genotypes. An additional possible explanation is that the resolution for both markers is different.

\section{Colony size}

We estimated the size of one colony on the basis of the number of fungus gardens and also considered the actual physical span of the colony in three dimensions. To test whether fungus gardens belong to the same colony we determined whether sample pairs with identical CO1 and ITS sequences were found in the same mound or also shared between mounds. For two sites ( $M O$ and $A R C$ ), we only found mound specific colonies and no shared colonies between mounds, but at site $E F$, we found colonies shared between different mounds. In total we found five cases in which colonies were shared between different mounds (Figure 6; 1A1D shared among four mounds, 1A1A shared among two mounds, 1A1B, shared among two mounds, 1A2A shared among two mounds and $1 \mathrm{C} 1 \mathrm{~A}$ shared among two mounds). If we take this into account for calculating the average colony size for this location this means that the average number of fungus gardens per colony is 4.9 instead of three. It also affects the size of the largest colony we found, which had eight fungus gardens, but will be 13 if we allow overlapping colonies between mounds. This could mean that the size of a Microtermes colony extends between different mounds, or, alternatively, that our resolution is insufficient to distinguish all colonies. On average we found 2.36 fungus gardens per mound of a unique combination. There were two cases with up to eight identical pairs at a single mound and 13 if we count overlapping colonies between mounds. Based on these numbers it is hard to estimate how many fungus gardens make up one Microtermes colony. An indication for a large colony size is the low number of royal chambers encountered during excavation, we only encountered two during the entire study. We also found a high number of different colonies at each mound with up to ten different colonies at mound Mn160 (Figure 6). This high cryptic diversity indicates that Microtermes spp. may play an important role in the functioning of the ecosystem (Hausberger et al. 2011). 


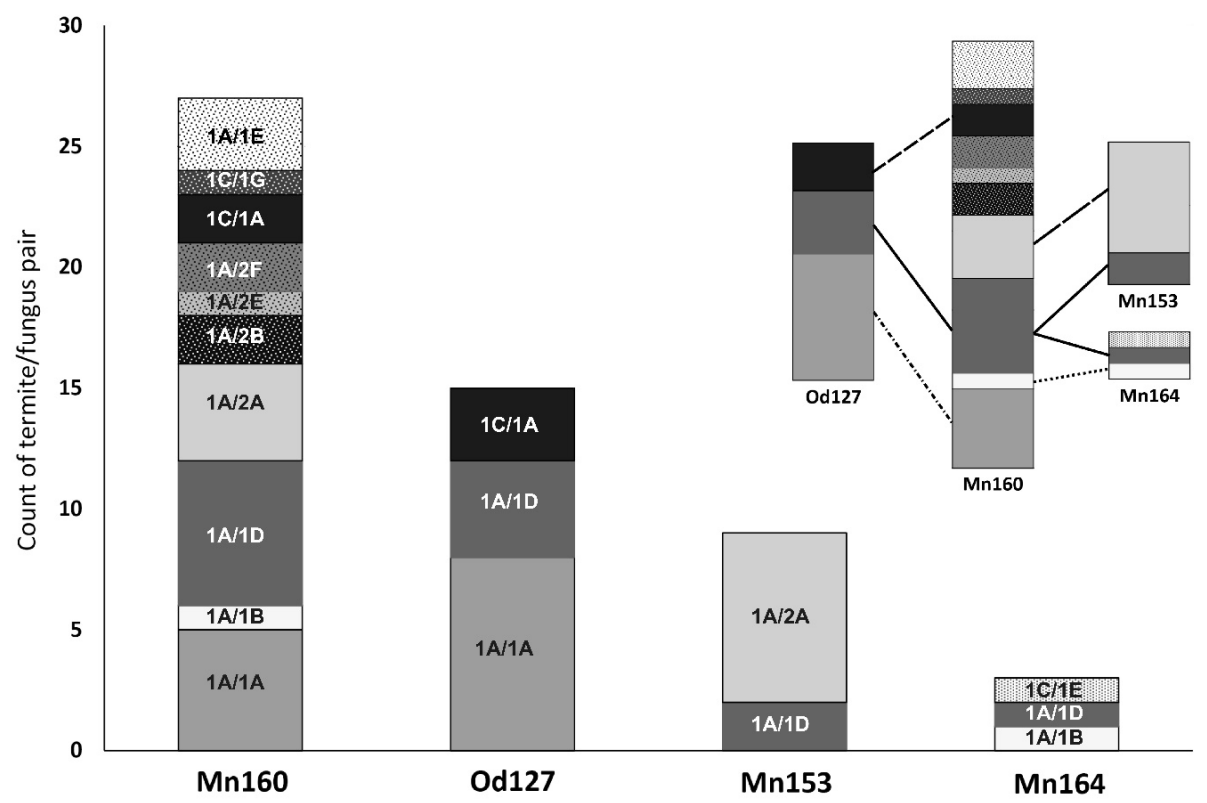

Figure 6 | Distribution of colonies over the different mounds at location EF. Each different colour and pattern indicates a unique type combination. Potential shared colonies between mounds are indicated in the top right panel. Colony $1 \mathrm{~A} / 1 \mathrm{D}$ is shared between all four mounds, colony $1 \mathrm{~A} / 1 \mathrm{~A}, 1 \mathrm{~A} / 1 \mathrm{~B}, 1 \mathrm{~A} / 2 \mathrm{~A}$ and $1 \mathrm{C} / 1 \mathrm{~A}$ at location $\mathrm{EF}$ are shared between two mounds. At the two remaining locations, $\mathrm{MO}$ and $\mathrm{ARC}$, each mound only has unique colonies which are not shared with the other mounds and are not shown in this picture.

We did not find shared occurrence of identical haplotype/genotype combinations at the different collection sites, which indicates that our method gives sufficient resolution to distinguish different colonies between different populations. We found five cases in which colonies were shared between different mounds at one collection site. These could be different colonies founded by sister queens or by female progeny from subsequent generations. If we assume these shared occurrences belong to a single colony it means that colonies may stretch as far as several hundreds of meters (Figure 2). This also implies that a major part of the colony may be found in the open field, which also explains why we only found two royal chambers during this study. For a more realistic estimate of colony size the sampling radius should be drastically increased and the open space in between mounds of other Macrotermitinae should be systematically excavated. Whether the density of Microtermes fungus gardens is higher in the large mounds of other species, and if so, what attracts Microtermes to those, is currently not known. 
Although our data offer enough resolution to study patterns of fungal diversity in relation to host diversity and how this is distributed in space, we cannot rule out that some identical combinations belong to different colonies. Neither can we rule out that a single termite colony contains genetically different fungi, since we assumed that a termite colony has a single fungal genotype, which has been found in all other studied species of fungus-growing termites, so far. Microsatellite data are needed to confirm that termites belong to the same colony and additional polymorphic markers are needed to confirm that the symbionts are the same clone.

\section{Host-specificity}

The five studied species of the genus Microtermes all have vertical symbiont transmission via the female reproductive (Johnson et al. 1981, Johnson 1981). Vertical transmission is a derived trait in the fungusgrowing termites, and horizontal transmission via the sexual spores produced by fruit bodies of the fungus is the ancestral state (Aanen et al. 2002). It is expected that sexual reproduction in the symbionts of Microtermes termites does not occur frequently, if it occurs at all. This is based on the absence of observations of fruit bodies for Microtermes symbionts (Johnson et al. 1981, Darlington 1994). However, it is possible that sexual reproduction could occur in these symbionts when they are cultivated by species of other genera of fungus-growing termites (Ancistrotermes, Allodontotermes, Acanthotermes and Synacanthotermes), since these share genetically identical or closely related symbionts with Microtermes (Aanen et al. 2002, Nobre, Kone, et al. 2011). Termitomyces medius, which is genetically very similar to Microtermes-associated symbionts in central Africa, does produce mushrooms when associated with species of Ancistrotermes (Aanen et al. 2002). It is not known whether Microtermes actively suppresses fruiting of their symbiont or if other factors prevent fruiting of the fungus.

Over time, strict vertical transmission is expected to lead to a tight host-symbiont relationship and reduced genetic variation in the symbiont (Herre et al. 1999, Frank 1997, Law and Dieckmann 1998). Our findings are not consistent with these theoretical expectations, since we found that a single termite haplotype (1A) could be associated with up to 12 different fungal genotypes from three of the four main clades. Fungi were not specific either to a single termite host. For example, fungal genotype 4 was associated with three different termite haplotypes from three of the four main clades. These findings show that host-switching must occur relatively often. A similar result has been 
found in fungus-growing ants which also have vertical transmission of their symbiont but occasionally horizontal transmission as well (Green, Mueller, and Adams 2002). Since Microtermes has vertical transmission of its symbiont and the symbionts do not fruit, the most likely way to obtain a different symbiont is by obtaining it from a neighbouring colony. Some of the Microtermes symbionts are shared with members from the genera Ancistrotermes, Acanthotermes, Allodontotermes and Synacanthotermes (Nobre, Kone, et al. 2011, Aanen et al. 2002). Fruit bodies associated with the nests of Ancistrotermes and Acanthotermes have frequently been found (Johnson et al. 1981, Koné et al. 2011, Koné et al. 2018). Theoretically a different symbiont could be obtained from basidiospores when fruiting occurs in these other genera. This does not seem very likely since there is little overlap in geographical range where species from these genera and Microtermes co-occur (Uys 2002). It is also not known over what distances basidiospores of Termitomyces can disperse. Studies on fungus garden establishment in Microtermes are limited and previous studies in a laboratory setting have not mentioned rates of successful fungus comb inoculation (Johnson et al. 1981, Johnson 1981). By pure chance, it is expected that establishment of a fungus garden in species with vertical symbiont transmission is not always successful, in which case the queen or the young workers may need to reobtain the symbiont from other colonies. We, therefore, expect that switching between fungal cultivars will most likely occur between generations because when the fungus is established within the colony the chance of it being outcompeted by an invading fungus is presumably low. In fungus-growing ants it has recently been observed that queens may lose their symbionts and are able to obtain a new symbiont from neighbouring fungus gardens (Howe, Schiøtt, and Boomsma 2018). Similarly, fungus-growing ants in the genus Cyphomyrmex are able to reobtain their symbiont if they accidentally lose it by stealing it from other colonies (Adams et al. 2000). Fungal crop theft has also been observed in fungus-growing ambrosia beetles (Hulcr and Cognato 2010). The high density of Microtermes colonies that we observed allows easy access to fungus gardens from other colonies in case a fungal symbiont is lost. However, we did not find any indication of symbiont exchange between different termite genera. Although all Microtermes sampled in this study were inhabiting mounds of either $M$. natalensis or Odontotermes spp. we never found Microtermes termites associated with the fungal symbiont of these genera. 
Microtermes has not been studied in great detail yet, probably because of its cryptic lifestyle. We aimed to resolve some of the basic questions about the biology of the genus Microtermes especially regarding colony size and host specificity. Microtermes certainly deserves more attention since it is one of the two cases in which vertical symbiont transmission has evolved in the Macrotermitinae and could serve as a model system for understanding the evolution of vertical transmission of symbionts. Vertical transmission is the main mode of symbiont transmission for Microtermes but we demonstrate that frequent horizontal exchange of genetically diverse symbionts occurs. Whether this horizontal exchange is the consequence of primary or secondary loss of the symbiont or whether termites actively search for a more suitable symbiont remains to be answered.

\section{Acknowledgements}

We would like to thank Mike and Brenda Wingfield, Wilhelm de Beer and the Forestry \& Agricultural Biotechnology Institute (FABI) for hosting us during the fieldwork in South Africa. We would like to thank Ben Auxier for helping us create Figure 2 and Figure 5. This research was funded by the Netherlands Organisation for Scientific Research (VICl; NWO 86514007).

\section{Supplementary document 1}

\begin{tabular}{ll}
\multicolumn{2}{l}{ PCR reaction mixture } \\
\hline 5x GoTaq buffer & $5 \mu \mathrm{l}$ \\
25mM MgCl2 & $2 \mu \mathrm{l}$ \\
10mM dNTP & $1 \mu \mathrm{l}$ \\
Forward primer & $1 \mu \mathrm{l}$ \\
Reverse primer & $1 \mu \mathrm{l}$ \\
GoTaq polymerase & $0.1 \mu \mathrm{l}$ \\
Template DNA $1: 10$ & $1 \mu \mathrm{l}$ \\
MQ water & $13.9 \mu \mathrm{l}$
\end{tabular}

Primer sequences and PCR programs

Fungal ITS (primers: ITS1f/ITS4)

ITS1f: 5'-CTTGGTCATTTAGAGGAAGTAA-3'

ITS4: 5'-TCCTCCGCTTATTGATATGC-3'

Initial denaturation at $94^{\circ} \mathrm{C}$ for $5 \mathrm{~min}$

35 cycles $\left(60 \mathrm{~s}\right.$ at $94^{\circ} \mathrm{C} ; \mathbf{6 0 s}$ at $53^{\circ} \mathrm{C} ; 60 \mathrm{~s}$ at $72^{\circ} \mathrm{C}$ ) 
Final elongation at $\mathbf{7 2}^{\circ} \mathrm{C}$ for $\mathbf{1 0} \mathbf{~ m i n}$

Termite COI (primers: TL1862/TH2877)

TL1862: 5'-TACTTCGTATTCGGAGCTTGA-3'

TH2877: 5'-TAGGTGTCGTGTAATACAATGTC-3'

Initial denaturation at $95^{\circ} \mathrm{C}$ for $\mathbf{5} \mathbf{~ m i n}$

35 cycles $\left(60 \mathrm{~s}\right.$ at $95^{\circ} \mathrm{C} ; 60 \mathrm{~s}$ at $50^{\circ} \mathrm{C} ; 60 \mathrm{~s}$ at $\left.72^{\circ} \mathrm{C}\right)$

Final elongation at $72^{\circ} \mathrm{C}$ for $\mathbf{1 0} \mathbf{~ m i n}$

\section{Supplementary tables}

Supplementary table 1 Termite haplotype COI accessions

\begin{tabular}{|l|l|l|}
\hline Isolate & Accession \# & Reference \\
\hline Microtermes sp. Haplotype 1A & MN166473 & This study \\
\hline Microtermes sp. Haplotype 1B & MN166474 & This study \\
\hline Microtermes sp. Haplotype 1C & MN166475 & This study \\
\hline Microtermes sp. Haplotype 2 & MN166476 & This study \\
\hline Microtermes sp. Haplotype 3A & MN166477 & This study \\
\hline Microtermes sp. Haplotype 3B & MN166478 & This study \\
\hline Microtermes sp. Haplotype 3C & MN166479 & This study \\
\hline Microtermes sp. Haplotype 4 & MN166480 & This study \\
\hline Microtermes sp. za9 & AY818069 & Aanen \& Eggleton 2005 \\
\hline Microtermes sp. za10 & AY818071 & Aanen \& Eggleton 2005 \\
\hline Microtermes sp. za31 & AY818074 & Aanen \& Eggleton 2005 \\
\hline Microtermes sp. za49 & AY818075 & Aanen \& Eggleton 2005 \\
\hline Microtermes sp. za135 & AY818087 & Aanen \& Eggleton 2005 \\
\hline Microtermes sp. za138 & AY818089 & Aanen \& Eggleton 2005 \\
\hline Ancistrotermes cavithorax dka34 & AY127712 & Aanen et al. 2002 \\
\hline Ancistrotermes cavithorax dka46 & AY127715 & Aanen et al. 2002 \\
\hline Ancistrotermes crucifer dka140 & AY127733 & Aanen et al. 2002 \\
\hline Pseudacanthotermes militaris dka134 & AY127731 & Aanen et al. 2002 \\
\hline
\end{tabular}


Supplementary table $2 \mid$ Termitomyces genotype ITS accessions

\begin{tabular}{|l|l|l|}
\hline Isolate & Accession \# & Reference \\
\hline Termitomyces sp. genotype 1A & MN161228 & This study \\
\hline Termitomyces sp. genotype 1B & MN161229 & This study \\
\hline Termitomyces sp. genotype 1C & MN161232 & This study \\
\hline Termitomyces sp. genotype 1D & MN161231 & This study \\
\hline Termitomyces sp. genotype 1E & MN161234 & This study \\
\hline Termitomyces sp. genotype 1F & MN161235 & This study \\
\hline Termitomyces sp. genotype 1G & MN161236 & This study \\
\hline Termitomyces sp. genotype 1H & MN161237 & This study \\
\hline Termitomyces sp. genotype 1I & MN161238 & This study \\
\hline Termitomyces sp. genotype 2A & MN161239 & This study \\
\hline Termitomyces sp. genotype 2B & MN161240 & This study \\
\hline Termitomyces sp. genotype 2C & MN161241 & This study \\
\hline Termitomyces sp. genotype 2D & MN161242 & This study \\
\hline Termitomyces sp. genotype 2E & MN161243 & This study \\
\hline Termitomyces sp. genotype 2F & MN161244 & This study \\
\hline Termitomyces sp. genotype 3A & MN161245 & This study \\
\hline Termitomyces sp. genotype 3B & MN161246 & This study \\
\hline Termitomyces sp. genotype 4 & MN161247 & This study \\
\hline Termitomyces sp. DKA2007 symbiont 20 & EF636922 & Aanen et al. 2007 \\
\hline Termitomyces sp. DKA2007 symbiont 21 & EF636923 & Aanen et al. 2007 \\
\hline Termitomyces sp. DKA2007 symbiont 22 & EF636924 & Aanen et al. 2007 \\
\hline Termitomyces sp. DKA2007 symbiont 23 & EF636925 & Aanen et al. 2007 \\
\hline Termitomyces sp. DKA2007 symbiont 24 & EF636926 & Aanen et al. 2007 \\
\hline Termitomyces sp. DKA2007 symbiont 25 & EF636927 & Aanen et al. 2007 \\
\hline Blastosporella zonata TJB8371 & EU708340 & Baroni et al. 2007 \\
\hline
\end{tabular}


Supplementary table $\mathbf{3}$ | Overview of all samples used with corresponding termite haplotype and genotype numbers

\begin{tabular}{|c|c|c|c|c|c|}
\hline Strain & Mound host & $\begin{array}{l}\text { Locatio } \\
\text { n }\end{array}$ & $\begin{array}{l}\text { Termite } \\
\text { haplotyp } \\
\text { e }\end{array}$ & $\begin{array}{l}\text { Fungal } \\
\text { genotyp } \\
\text { e }\end{array}$ & $\begin{array}{l}\text { Termite/Fung } \\
\text { us }\end{array}$ \\
\hline Mi127 005 & Odontotermes sp. & $\mathrm{EF}$ & $1 \mathrm{~A}$ & $1 \mathrm{~A}$ & $1 \mathrm{~A} / 1 \mathrm{~A}$ \\
\hline Mi127008 & Odontotermes sp. & $\mathrm{EF}$ & $1 \mathrm{~A}$ & $1 \mathrm{~A}$ & $1 \mathrm{~A} / 1 \mathrm{~A}$ \\
\hline Mi127 010 & Odontotermes sp. & $\mathrm{EF}$ & $1 \mathrm{C}$ & $1 \mathrm{~A}$ & $1 \mathrm{C} / 1 \mathrm{~A}$ \\
\hline Mi127 011 & Odontotermes sp. & $\mathrm{EF}$ & $1 \mathrm{~A}$ & $1 \mathrm{~A}$ & $1 \mathrm{~A} / 1 \mathrm{~A}$ \\
\hline Mi127 013 & Odontotermes sp. & $\mathrm{EF}$ & $1 \mathrm{~A}$ & $1 \mathrm{~A}$ & $1 \mathrm{~A} / 1 \mathrm{~A}$ \\
\hline Mi127 016 & Odontotermes sp. & $\mathrm{EF}$ & $1 \mathrm{~A}$ & $1 \mathrm{D}$ & 1A/1D \\
\hline Mi127 017 & Odontotermes sp. & $\mathrm{EF}$ & $1 \mathrm{~A}$ & $1 \mathrm{~A}$ & $1 \mathrm{~A} / 1 \mathrm{~A}$ \\
\hline Mi127 018 & Odontotermes sp. & EF & $1 \mathrm{~A}$ & 1D & 1A/1D \\
\hline Mi127 021 & Odontotermes sp. & $\mathrm{EF}$ & $1 \mathrm{C}$ & $1 \mathrm{~A}$ & $1 \mathrm{C} / 1 \mathrm{~A}$ \\
\hline Mi127 022 & Odontotermes sp. & $\mathrm{EF}$ & $1 \mathrm{~A}$ & $1 \mathrm{~A}$ & $1 \mathrm{~A} / 1 \mathrm{~A}$ \\
\hline Mi127 023 & Odontotermes sp. & EF & $1 \mathrm{C}$ & $1 \mathrm{~A}$ & $1 \mathrm{C} / 1 \mathrm{~A}$ \\
\hline Mi127 024 & Odontotermes sp. & $\mathrm{EF}$ & $1 \mathrm{~A}$ & 1D & 1A/1D \\
\hline Mi127 025 & Odontotermes sp. & $\mathrm{EF}$ & $1 \mathrm{~A}$ & 1D & 1A/1D \\
\hline Mi127 026 & Odontotermes sp. & EF & $1 \mathrm{~A}$ & $1 \mathrm{~A}$ & $1 \mathrm{~A} / 1 \mathrm{~A}$ \\
\hline Mi127 027 & Odontotermes sp. & $\mathrm{EF}$ & $1 \mathrm{~A}$ & $1 \mathrm{~A}$ & $1 \mathrm{~A} / 1 \mathrm{~A}$ \\
\hline Mi153 001 & M. natalensis & $\mathrm{EF}$ & $1 \mathrm{~A}$ & $2 \mathrm{~A}$ & $1 \mathrm{~A} / 2 \mathrm{~A}$ \\
\hline Mi153002 & M. natalensis & EF & $1 \mathrm{~A}$ & $2 \mathrm{~A}$ & $1 \mathrm{~A} / 2 \mathrm{~A}$ \\
\hline Mi153003 & M. natalensis & $\mathrm{EF}$ & $1 \mathrm{~A}$ & $2 \mathrm{~A}$ & $1 \mathrm{~A} / 2 \mathrm{~A}$ \\
\hline Mi153004 & M. natalensis & EF & $1 \mathrm{~A}$ & $2 \mathrm{~A}$ & $1 \mathrm{~A} / 2 \mathrm{~A}$ \\
\hline Mi153006 & M. natalensis & $\mathrm{EF}$ & $1 \mathrm{~A}$ & 1D & 1A/1D \\
\hline Mi153007 & M. natalensis & $\mathrm{EF}$ & $1 \mathrm{~A}$ & $1 \mathrm{D}$ & 1A/1D \\
\hline Mi153 008 & M. natalensis & EF & $1 \mathrm{~A}$ & $2 \mathrm{~A}$ & $1 \mathrm{~A} / 2 \mathrm{~A}$ \\
\hline Mi153009 & M. natalensis & $\mathrm{EF}$ & $1 \mathrm{~A}$ & $2 \mathrm{~A}$ & $1 \mathrm{~A} / 2 \mathrm{~A}$ \\
\hline Mi153012 & M. natalensis & $\mathrm{EF}$ & $1 \mathrm{~A}$ & $2 \mathrm{~A}$ & $1 \mathrm{~A} / 2 \mathrm{~A}$ \\
\hline Mi160 001 & M. natalensis & EF & $1 \mathrm{~A}$ & 1D & 1A/1D \\
\hline Mi160 003 & M. natalensis & $\mathrm{EF}$ & $1 \mathrm{~A}$ & $2 \mathrm{E}$ & $1 \mathrm{~A} / 2 \mathrm{E}$ \\
\hline Mi160 004 & M. natalensis & EF & $1 \mathrm{~A}$ & $1 \mathrm{~A}$ & $1 \mathrm{~A} / 1 \mathrm{~A}$ \\
\hline Mi160008 & M. natalensis & $\mathrm{EF}$ & $1 \mathrm{~A}$ & $1 \mathrm{D}$ & $1 \mathrm{~A} / 1 \mathrm{D}$ \\
\hline Mi160 009 & M. natalensis & EF & $1 \mathrm{C}$ & $1 \mathrm{~A}$ & $1 \mathrm{C} / 1 \mathrm{~A}$ \\
\hline Mi160 010 & M. natalensis & EF & $1 \mathrm{~A}$ & $2 \mathrm{~A}$ & $1 \mathrm{~A} / 2 \mathrm{~A}$ \\
\hline Mi160 011 & M. natalensis & $\mathrm{EF}$ & $1 \mathrm{~A}$ & $2 \mathrm{~A}$ & $1 \mathrm{~A} / 2 \mathrm{~A}$ \\
\hline Mi160 017 & M. natalensis & $\mathrm{EF}$ & $1 \mathrm{~A}$ & $1 \mathrm{G}$ & $1 \mathrm{~A} / 1 \mathrm{G}$ \\
\hline
\end{tabular}




\begin{tabular}{|c|c|c|c|c|c|}
\hline Mi160 018 & M. natalensis & $\mathrm{EF}$ & $1 \mathrm{~A}$ & $1 \mathrm{~A}$ & $1 \mathrm{~A} / 1 \mathrm{~A}$ \\
\hline Mi160 019 & M. natalensis & EF & $1 \mathrm{~A}$ & $1 \mathrm{~A}$ & $1 \mathrm{~A} / 1 \mathrm{~A}$ \\
\hline Mi160 021 & M. natalensis & EF & $1 \mathrm{~A}$ & $2 \mathrm{~F}$ & $1 \mathrm{~A} / 2 \mathrm{~F}$ \\
\hline Mi160 022 & M. natalensis & $\mathrm{EF}$ & $1 \mathrm{~A}$ & $2 \mathrm{~A}$ & $1 \mathrm{~A} / 2 \mathrm{~A}$ \\
\hline Mi160 026 & M. natalensis & $\mathrm{EF}$ & $1 \mathrm{~A}$ & $1 \mathrm{D}$ & $1 \mathrm{~A} / 1 \mathrm{D}$ \\
\hline Mi160 027 & M. natalensis & $\mathrm{EF}$ & $1 \mathrm{~A}$ & $1 \mathrm{G}$ & $1 \mathrm{~A} / 1 \mathrm{G}$ \\
\hline Mi160 029 & M. natalensis & $\mathrm{EF}$ & $1 \mathrm{~A}$ & $2 \mathrm{~F}$ & $1 \mathrm{~A} / 2 \mathrm{~F}$ \\
\hline Mi160030 & M. natalensis & $\mathrm{EF}$ & $1 \mathrm{~A}$ & $1 \mathrm{D}$ & 1A/1D \\
\hline Mi160 031 & M. natalensis & EF & $1 \mathrm{~A}$ & $2 B$ & $1 \mathrm{~A} / 2 \mathrm{~B}$ \\
\hline Mi160 032 & M. natalensis & $\mathrm{EF}$ & $1 \mathrm{~A}$ & $1 \mathrm{D}$ & 1A/1D \\
\hline Mi160034 & M. natalensis & $\mathrm{EF}$ & $1 \mathrm{C}$ & $1 \mathrm{~A}$ & $1 \mathrm{C} / 1 \mathrm{~A}$ \\
\hline Mi160035 & M. natalensis & $\mathrm{EF}$ & $1 \mathrm{~A}$ & $1 \mathrm{D}$ & $1 \mathrm{~A} / 1 \mathrm{D}$ \\
\hline Mi160 041 & M. natalensis & EF & $1 \mathrm{~A}$ & $1 \mathrm{~A}$ & $1 \mathrm{~A} / 1 \mathrm{~A}$ \\
\hline Mi160 048 & M. natalensis & EF & $1 \mathrm{C}$ & 11 & $1 \mathrm{C} / 1 \mathrm{I}$ \\
\hline Mi160 049 & M. natalensis & $\mathrm{EF}$ & $1 \mathrm{~A}$ & $2 \mathrm{~B}$ & $1 \mathrm{~A} / 2 \mathrm{~B}$ \\
\hline Mi160053 & M. natalensis & $\mathrm{EF}$ & $1 \mathrm{~A}$ & $2 A$ & $1 \mathrm{~A} / 2 \mathrm{~A}$ \\
\hline Mi160058 & M. natalensis & EF & $1 \mathrm{~A}$ & $1 \mathrm{~A}$ & $1 \mathrm{~A} / 1 \mathrm{~A}$ \\
\hline Mi160 061 & M. natalensis & EF & $1 \mathrm{~A}$ & $1 \mathrm{~B}$ & 1A/1B \\
\hline Mi160063 & M. natalensis & $\mathrm{EF}$ & $1 \mathrm{~A}$ & $1 \mathrm{G}$ & $1 \mathrm{~A} / 1 \mathrm{G}$ \\
\hline Mi164 002 & M. natalensis & $\mathrm{EF}$ & $1 \mathrm{~A}$ & $1 \mathrm{D}$ & 1A/1D \\
\hline Mi164003 & M. natalensis & $\mathrm{EF}$ & $1 \mathrm{~A}$ & $1 \mathrm{~B}$ & 1A/1B \\
\hline Mi164005 & M. natalensis & EF & $1 \mathrm{C}$ & $1 \mathrm{G}$ & $1 \mathrm{C} / 1 \mathrm{G}$ \\
\hline Mi132001 & M. natalensis & $\mathrm{MO}$ & $3 C$ & 4 & $3 \mathrm{C} / 4$ \\
\hline Mi132 002 & M. natalensis & $\mathrm{MO}$ & $1 \mathrm{~A}$ & $1 \mathrm{Ex}$ & 1A/1Ex \\
\hline Mi132003 & M. natalensis & $\mathrm{MO}$ & 4 & $1 \mathrm{~K}$ & $4 / 1 \mathrm{~K}$ \\
\hline Mi132004 & M. natalensis & $\mathrm{MO}$ & 4 & $1 \mathrm{C}$ & $4 / 1 C$ \\
\hline Mi132005 & M. natalensis & $\mathrm{MO}$ & 4 & $1 \mathrm{E}$ & $4 / 1 E$ \\
\hline Mi165002 & M. natalensis & $\mathrm{MO}$ & $3 B$ & $1 \mathrm{~J}$ & $3 \mathrm{~B} / 1 \mathrm{~J}$ \\
\hline Mi165 003 & M. natalensis & $\mathrm{MO}$ & $1 \mathrm{~A}$ & $1 \mathrm{H}$ & $1 \mathrm{~A} / 1 \mathrm{H}$ \\
\hline Mi165 004 & M. natalensis & $\mathrm{MO}$ & $1 \mathrm{~A}$ & $2 \mathrm{D}$ & $1 \mathrm{~A} / 2 \mathrm{D}$ \\
\hline Mi165005 & M. natalensis & $\mathrm{MO}$ & 2 & $2 \mathrm{E}$ & $2 / 2 E$ \\
\hline Mi165007 & M. natalensis & $\mathrm{MO}$ & 2 & $2 \mathrm{C}$ & $2 / 2 \mathrm{C}$ \\
\hline Mi165 009 & M. natalensis & $\mathrm{MO}$ & $1 \mathrm{~A}$ & $1 \mathrm{H}$ & $1 \mathrm{~A} / 1 \mathrm{H}$ \\
\hline Mi165010 & M. natalensis & $\mathrm{MO}$ & $1 \mathrm{C}$ & $1 \mathrm{Dx}$ & 1C/1Dx \\
\hline Mi165 011 & M. natalensis & $\mathrm{MO}$ & $3 C$ & $2 \mathrm{C}$ & $3 \mathrm{C} / 2 \mathrm{C}$ \\
\hline Mi166 001 & M. natalensis & $\mathrm{MO}$ & 4 & 4 & $4 / 4$ \\
\hline
\end{tabular}




\begin{tabular}{|c|c|c|c|c|c|}
\hline Mi166 002 & M. natalensis & $\mathrm{MO}$ & 4 & 4 & $4 / 4$ \\
\hline Mi166 003 & M. natalensis & $\mathrm{MO}$ & $3 B$ & $3 \mathrm{~A}$ & $3 B / 3 A$ \\
\hline Mi166 004 & M. natalensis & $\mathrm{MO}$ & $3 \mathrm{~A}$ & $3 \mathrm{~A}$ & $3 \mathrm{~A} / 3 \mathrm{~A}$ \\
\hline Mi166 005 & M. natalensis & $\mathrm{MO}$ & 4 & 4 & $4 / 4$ \\
\hline Mi166 008 & M. natalensis & $\mathrm{MO}$ & $3 C$ & $3 B$ & $3 \mathrm{C} / 3 \mathrm{~B}$ \\
\hline Mi166 009 & M. natalensis & $\mathrm{MO}$ & $3 A$ & $2 \mathrm{~A}$ & $3 \mathrm{~A} / 2 \mathrm{~A}$ \\
\hline Mi166 011 & M. natalensis & $\mathrm{MO}$ & $3 A$ & $3 \mathrm{~A}$ & $3 \mathrm{~A} / 3 \mathrm{~A}$ \\
\hline Mi167 001 & Odontotermes sp. & ARC & $1 \mathrm{~A}$ & 4 & $1 \mathrm{~A} / 4$ \\
\hline Mi167 002 & Odontotermes sp. & ARC & 1B & $1 \mathrm{~F}$ & $1 \mathrm{~B} / 1 \mathrm{~F}$ \\
\hline Mi167003 & Odontotermes sp. & ARC & $1 \mathrm{~A}$ & 4 & $1 \mathrm{~A} / 4$ \\
\hline Mi167 005 & Odontotermes sp. & ARC & $1 \mathrm{~A}$ & 4 & $1 \mathrm{~A} / 4$ \\
\hline Mi167 006 & Odontotermes sp. & ARC & $1 \mathrm{~A}$ & 4 & $1 \mathrm{~A} / 4$ \\
\hline Mi167 007 & Odontotermes sp. & ARC & $1 \mathrm{~A}$ & 4 & $1 \mathrm{~A} / 4$ \\
\hline Mi167 008 & Odontotermes sp. & ARC & $1 \mathrm{~A}$ & 4 & $1 \mathrm{~A} / 4$ \\
\hline Mi167 009 & Odontotermes sp. & ARC & $1 \mathrm{~A}$ & 4 & $1 \mathrm{~A} / 4$ \\
\hline Mi167011 & Odontotermes sp. & ARC & $1 \mathrm{~A}$ & 4 & $1 \mathrm{~A} / 4$ \\
\hline Mi167 012 & Odontotermes sp. & ARC & $1 \mathrm{~B}$ & $1 \mathrm{~F}$ & $1 \mathrm{~B} / 1 \mathrm{~F}$ \\
\hline
\end{tabular}




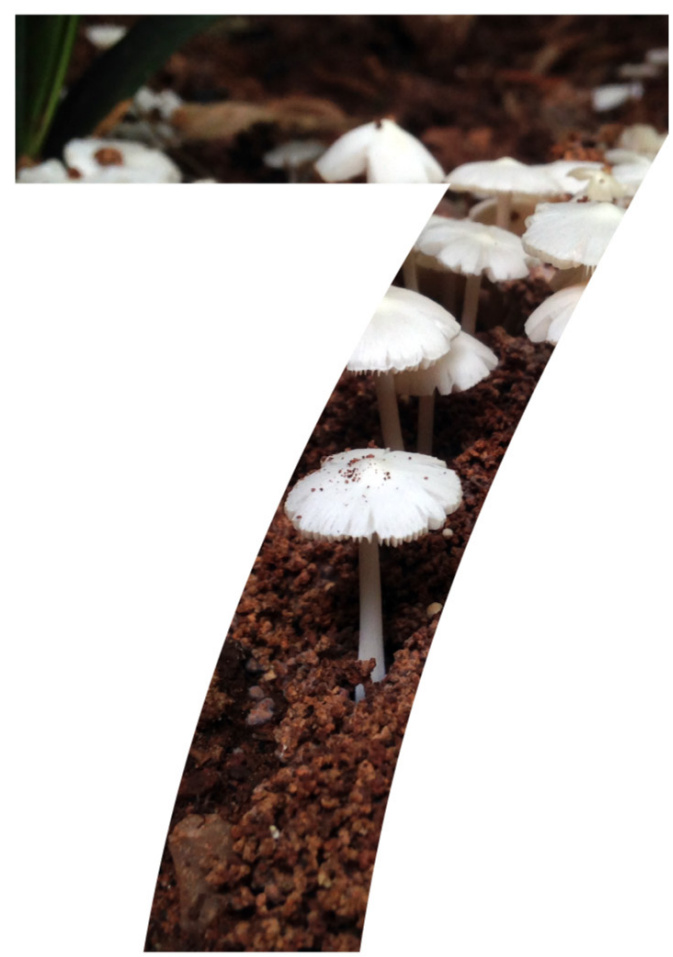

Termitomyces cryptogamus sp. nov. associated with Macrotermes natalensis in Africa

Lennart J.J. van de Peppel Z. Wilhelm de Beer Duur K. Aanen Ben Auxier 


\section{Abstract}

A new species of Termitomyces is described from Africa, symbiotic with the termite Macrotermes natalensis. As there are no records of field collected basidiocarps within this lineage, traditional basidiocarp based morphological taxonomy is not practical. While basidiocarps can rarely be obtained from incubation of fungal comb fragments, their practical use for taxonomical purposes is limited. Therefore, the species is described based on an ITS nucleotide sequence, with comparisons to an asexual culture. Based on samples with similar ITS sequences, this species is likely associated with multiple termite hosts across a large part of Africa.

* The species name in this chapter will be validated at a later stage in accordance with the International Code of Nomenclature for Algae, Fungi and Plants. 


\section{Introduction}

In Africa and Asia a subfamily of the Termitidae, the Macrotermitinae, live in obligate symbiosis with members of the basidiomycete genus Termitomyces (Lyophyllaceae). The fungus resides inside the termite nest in the so-called "fungus gardens", with mushrooms being periodically triggered by rain or potentially the health of the nest (Koné et al. 2011). The mushrooms range in size and are generally medium to large although some species, such as T. microcarpus (Berk. \& Broome) R. Heim, produce hundreds of small mushrooms. Traditionally, taxonomy in this genus has been based on basidiocarp morphology, with recent addition of molecular evidence (Frøslev et al. 2003, Mossebo et al. 2017).

During research of termites and their associated fungi in Africa, a lineage was found that associates with Macrotermes bellicosus and $M$. subhyalinus when found in northern Africa, but only with $M$. natalensis when found in South Africa (Nobre, Fernandes, et al. 2011). Surprisingly, sampling over ten years has not resulted in the discovery of mature basidiocarps of this species from any of its termite hosts, frustrating efforts for naming this species. Rarely, while excavating termite mounds, mushroom primordia are recovered (De Fine Licht, Andersen, and Aanen 2005, Vreeburg et al. 2020), and these can occasionally be incubated under laboratory conditions in the absence of termite workers. Presumably due to this laboratory incubation the mushrooms are misshapen, as the appearance is not consistent with other known Termitomyces species. This may be similar to development that occurs in the production of "Enoki" mushroom from Flammulina velutipes which causes long thin stipes, smaller caps and pale colouration (Kües and Navarro-González 2015). There is a need to provide a name to this well-studied fungus, but the lack of basidiocarps prevents comparisons with published literature descriptions (Botha and Eicker 1991b), which has led to an argument for using readily obtained asexual cultures as a stable type against which to compare (Makonde et al. 2013).

As extensive comparisons of ITS sequences from herbarium material from South Africa recovered no matches with our M. natalensis symbiont (van de Peppel et al. in prep), we provide a description based on sequence identity and asexual morphology. While there is a previous report of asexual characteristics from South African Termitomyces, only mounds from which basidiocarps could be found were used, and thus M. natalensis mounds were apparently not sampled (Botha and Eicker 1991b). As records of basidiocarps are lacking, asexual cultures are a logical solution to this taxonomic issue. Three previous publications 
addressed differences between species in asexual cultures, and while interspecific differences were found, asexual characteristics alone were not considered sufficient to delineate species (Botha and Eicker 1991a, b, Tibuhwa 2012).

Recently an ecological study of the symbionts of Macrotermes in Kenya found results consistent with previous sampling of this lineage (Vesala et al. 2017). Vesala et al. recovered the same fungal species as the one collected from South Africa (De Fine Licht, Andersen, and Aanen 2005, Nobre, Fernandes, et al. 2011, Vreeburg et al. 2020) from mounds of $M$. bellicosus, $M$. herus, M. jeanneli, M. michaelseni and $M$. subhyalinus. Vesala et al. included related ITS sequences from Genbank, including symbionts of $M$. natalensis and $M$. bellicosus, finding that these formed a supported clade. These sequences form two well supported groups with $97 \%$ and $98.5 \%$ support respectively, with the exception of a single sequence (Genbank id: AF357024), isolated from an unidentified Macrotermes species. Based on these results we describe the species based on a fungal isolate from South Africa symbiotic with $M$. natalensis for which the fungal genome has been published (Poulsen et al. 2014).

\section{Materials \& Methods}

\section{Samples and Isolates}

Details regarding sampling, isolation and subsequent protoplasting of this specific P5 culture are found in Poulsen et al. 2014 and Nobre et al. 2014. Briefly, a termite mound was opened, and nodules of asexual spores were carefully removed from the fungal combs without soil contamination. These nodules were placed on agar plates without antibiotics to establish hyphal cultures. A dried specimen and living culture representing the holotype and ex-holotype, respectively, were deposited to the CBS collection of the Westerdijk Fungal Biodiversity Institute, Utrecht, the Netherlands

\section{DNA extraction, PCR amplification, and sequencing}

Genomic DNA was extracted from an asexual culture using the cetyltrimethylammonium bromide (CTAB) protocol using mycelium and spores scraped from a petri dish. The nuclear ribosomal region containing the internal transcribed spacer 1, 5.8S and the internal transcribed spacer 2 was amplified using a standard PCR reaction using Promega GoTaq polymerase using the fungal specific primer ITS1F and the general reverse primer ITS4 (White et al. 1990, Gardes and Bruns 
1993). The 28S Large Ribosomal Subunit (LSU) was amplified using primer LROR and LR5 (Vilgalys and Hester 1990).

As no basidiocarps have been reported from $M$. natalensis termite mounds, we also surveyed herbarium samples from the South African National Collection of Fungi (PREM) and Schweickerdt Herbarium (PRUM) (van de Peppel et al. in prep).

\section{Sequence alignment and phylogenetic reconstruction}

We used a previously published set of ITS sequences (Vesala et al. 2017) with the addition of six sequences from Termitomyces symbiotic with $M$. natalensis from Genbank and a sequence generated from the specimen we designated as the type for the new species. Sequences were aligned using the web server of the alignment software MAFFT version 7 using default settings (Katoh and Standley 2013). Maximum likelihood trees were reconstructed using the web server of IQ-TREE version 2.0.6 using default settings (Trifinopoulos et al. 2016).

\section{Culture and Microscopy}

Cultures were maintained on MYA (20g Malt Extract, $2 \mathrm{~g}$ Yeast Extract, 1 $\mathrm{L} \mathrm{H}_{2} \mathrm{O}$ ), and incubated at $25^{\circ} \mathrm{C}$. Microscopy was performed on a Zeiss Axio Imager A1 with 63X objective lens under DIC optics.

\section{Taxonomy}

Termitomyces cryptogamus Van de Peppel sp. nov. Fig. 1

SEXUAL STATE-not observed.

ASEXUAL STATE-Growth of colonies on MYA medium reaching 5--6 cm in diameter in 3 weeks at $25^{\circ} \mathrm{C}$, somewhat faster at $30^{\circ} \mathrm{C}$. Growth consists of white hyphae mostly submerged in agar, with large $(1-2 \mathrm{~mm})$ clusters of asexual spores produced on the agar surface. Conidia produced are highly variable in size (10-100 mm in length) and shape, with 2-5 nuclei per spore. Dikaryotic colonies consistently produce dikaryotic conidia.

ETYMOLOGY: cryptogamus, referring to the hidden marriages of a genetically well-mixed species without recorded basidiocarps

DIAGNOSIS- In the absence of basidiocarps, T. cryptogamus cannot be distinguished readily from closely related known or unknown Termitomyces species based on its asexual characters. However, phylogenetic analyses of ITS and LSU sequences clearly separates a well-supported clade that includes the holotype of $T$. cryptogamus and several other strains obtained from fungal combs of M. natalensis in South Africa, as well as from those of M. bellicosus, 
M. herus, M. jeanneli, M. michaelseni and M. subhyalinus in Cameroon, Kenya, Senegal and Ivory Coast.

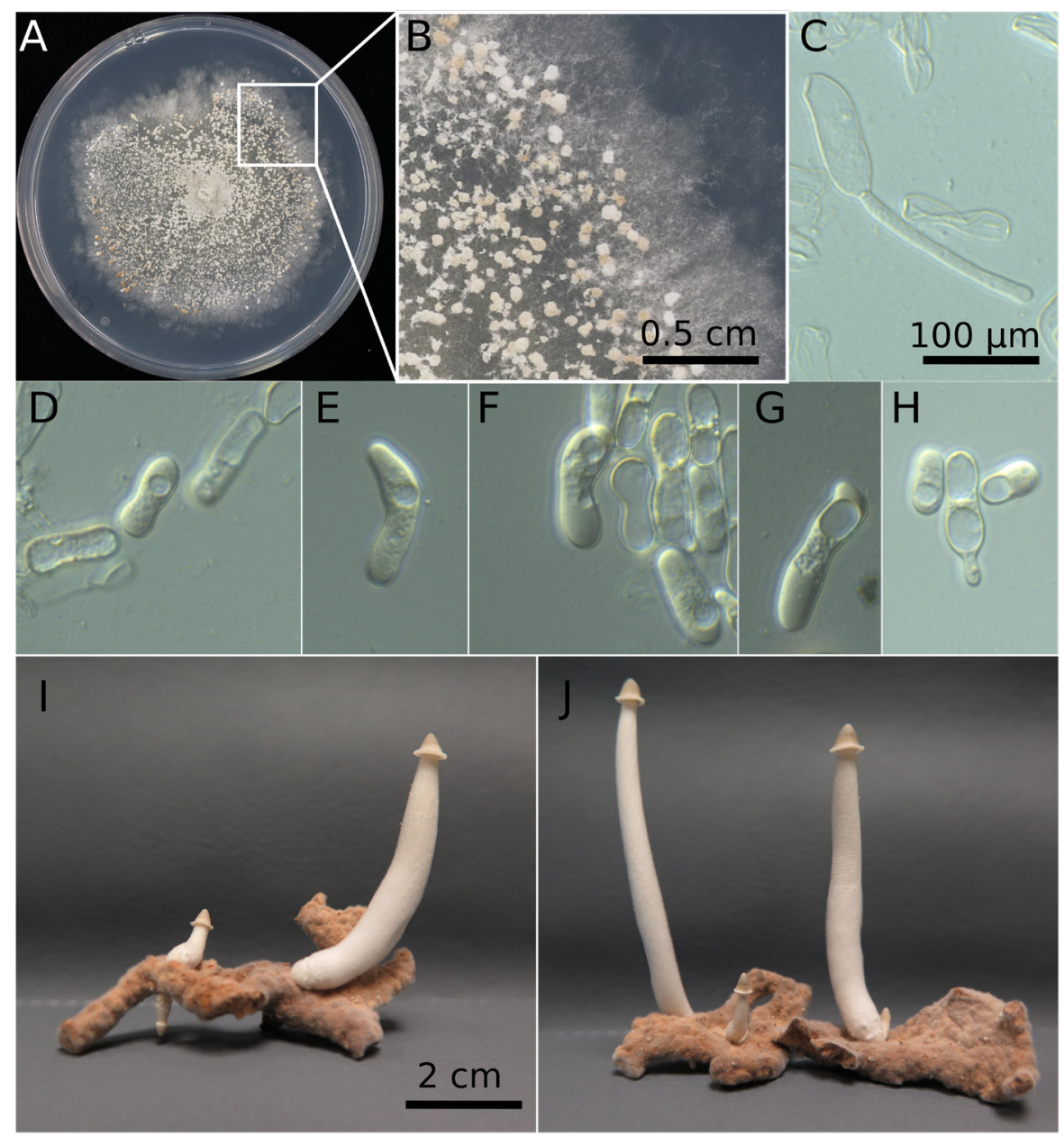

Figure 1 | Morphology of Termitomyces cryptogamus sp. nov. A) Growth on Malt Yeast Agar showing abundant nodule formation of asexual conidia. B) Close up view of $\mathrm{A}$. C-H) variable morphology of conidia. (-J) morphology of mushrooms on fragments of excavated fungus garden comb produced after incubation for 5-10 days. Note small caps $(<1 \mathrm{~cm})$ which still produce viable basidiospores. 


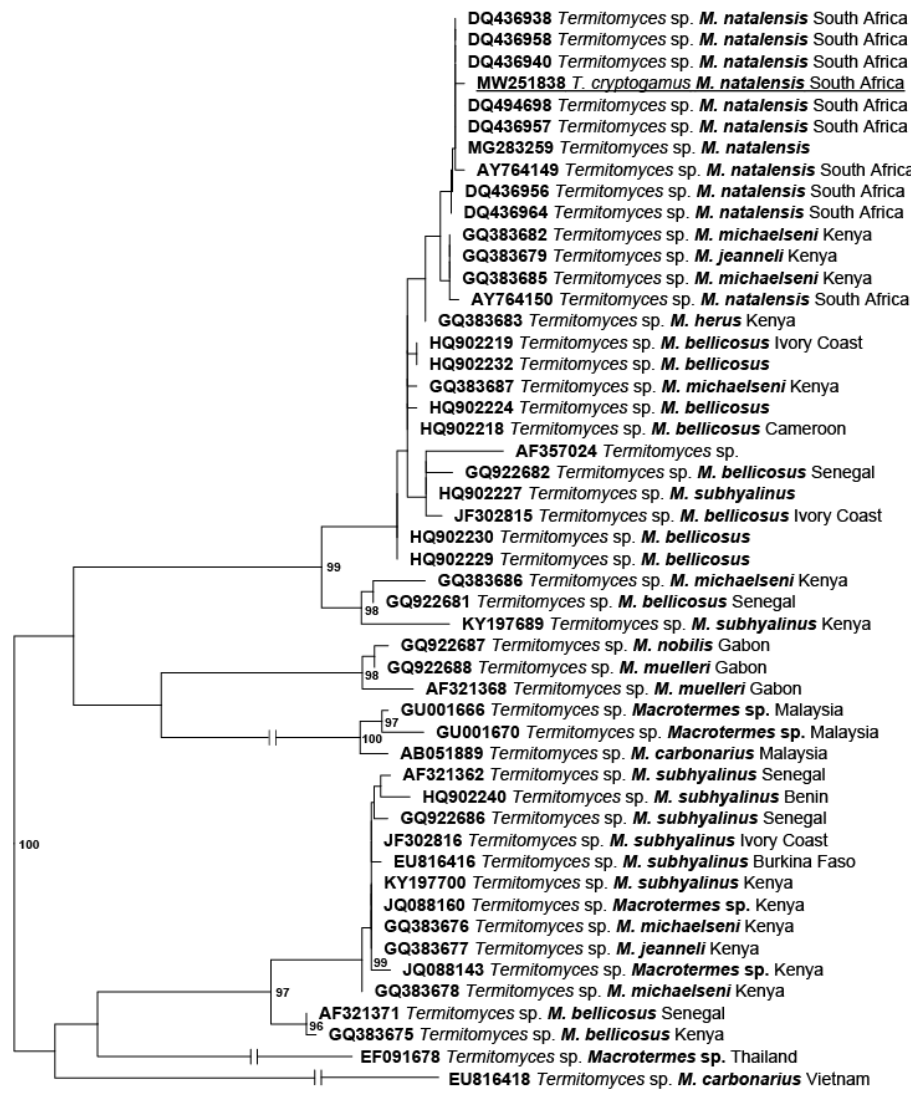

Termitomyces

cryptogamus sp. nov.

Group 1 sensu Vesala et al. 2017

Group 3 sensu Vesala et al 2017

Group 4 sensu Vesala et al. 2017

Group 2 sensu Vesala et al. 2017

Group 5 sensu Vesala et al. 2017

Group 6 sensu Vesala et al. 2017

Group 7 sensu Vesala et al. 2017

Group 9 sensu Vesala et al. 2017 Group 8 sensu Vesala et al. 2017

Figure 2 | Maximum likelihood tree based on ITS1-5.8S-ITS2 sequences of $T$. cryptogamus and allied Macrotermes symbionts with their host termite species (in bold) and their collection location. Outgroups were removed to increase readability of the tree. Numbers at the nodes indicate ultrafast bootstrap values, only significant node values $>95$ are displayed. Species delimitation groups with $97 \%$ ITS identity sensu Vesala et al. 2017 are displayed on the right side of the tree. A sequence of the T. cryptogamus type specimen P5 is underlined and indicated by an asterisk. 


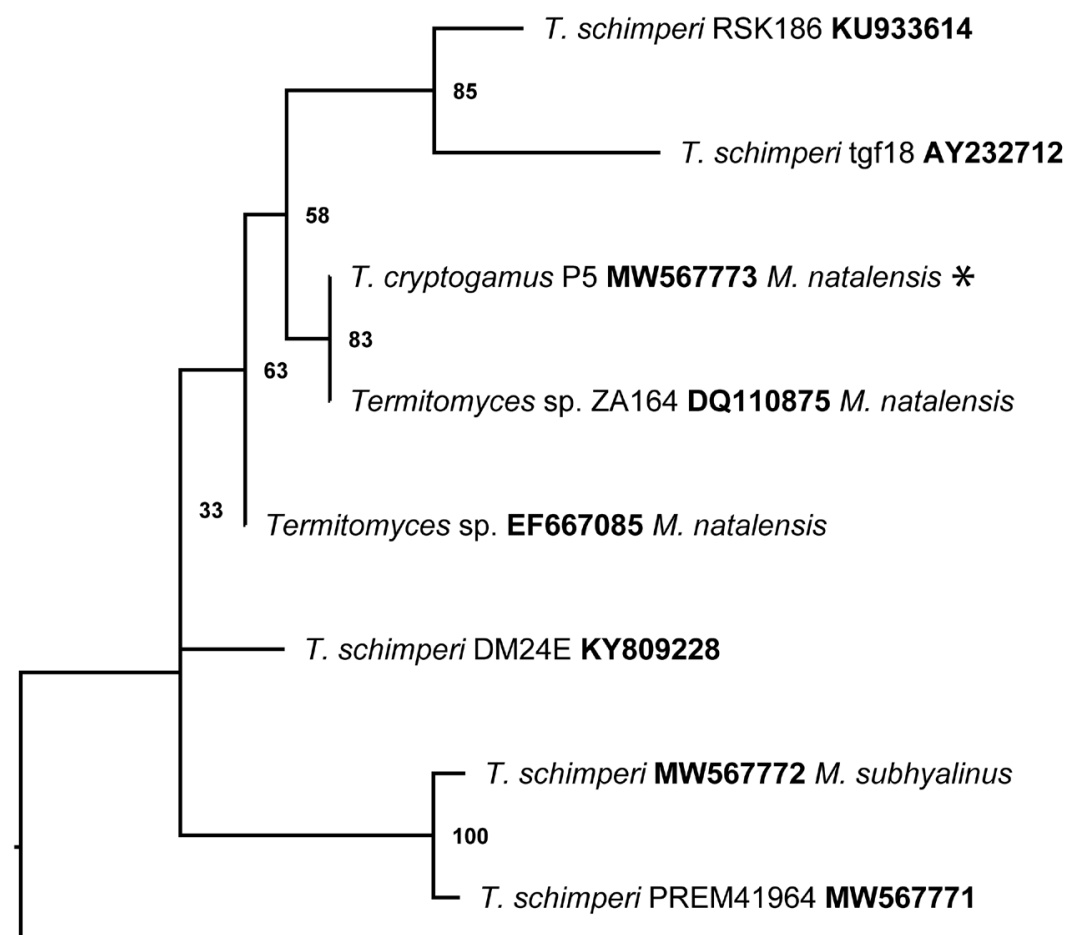

Termitomyces sp. AB073526 Odontotermes sp.

0.006

Figure 3 | Phylogenetic tree based on LSU sequences for T. cryptogamus and publicly available LSU sequences of $T$. schimperi, a common Macrotermes symbiont. Sequence of AB073526 used as an outgroup to root the tree. Node values indicate bootstrap support.

\section{Results}

The ITS sequence of our isolate P5 of Termitomyces cryptogamus sp. nov. placed it inside Group 1 of Vesala et al., 2017 (Fig 2). This group includes fungal individuals symbiotic with $M$. natalensis, $M$. bellicosus, M. subhyalinus, M. michaelseni, and M. jeanneli. Except for $M$. natalensis, these termite species are also found with closely related sister species to Termitomyces cryptogamus sp. nov.

To compare against other common Macrotermes mound symbionts, we also extracted DNA from samples of $T$. schimperi. However, repeated PCR amplifications of the ITS region were not successful. We were able to amplify LSU sequences from both $T$. cryptogamus sp. nov, as well as from herbarium samples of $T$. schimperi 
(PREM41964) (Figure 3). The LSU sequence of our P5 isolate was identical to a previously reported LSU sequence of a fungal symbiont of a $M$. natalensis mound. The commonly recovered Macrotermes symbiont, T. schimperi, was not monophyletic according to the LSU sequence relationships.

Microscopic investigations showed highly variable morphology of conidia harvested from laboratory grown cultures (Figure $1 \mathrm{C}-\mathrm{H}$ ). This limits morphological comparison with asexual cultures of other Termitomyces species.

\section{Discussion}

There is accumulating evidence, both direct and indirect, that sexual reproduction occurs in $T$. cryptogamus. First sexual reproduction between strains associated with $M$. natalensis was reconstructed to occur sufficiently frequently (at least 100 sexual events per generation) to explain the observed signature of free recombination (de Fine Licht, Boomsma, and Aanen 2006). Second, mating tests between homokaryons retrieved from heterokaryons demonstrated that the $M$. natalensis-associated strains belong to a single biological species (Nobre et al. 2014). Finally, in vitro, strains associated with M. natalensis were observed to produce mushrooms and viable basidiospores (De Fine Licht, Andersen, and Aanen 2005, Vreeburg et al. 2020). These findings make it all the more enigmatic that mushrooms have not been found in nature. A speculative hypothesis is that sexual reproduction of this species occurs belowground, synchronously with alate dispersal.

Nevertheless, the recovery of only after prolonged laboratory incubation prevents their use as a morphological type specimen, as presumably the incubation conditions have a large influence on the resulting morphology. Additionally, the infrequency and unpredictability of these mushroom primordia in nests precludes their use for identification. Further, the asexual spores produced are highly polymorphic, and thus cannot be used for reliable identification with this group. As such, molecular markers provide the only reliable way to identify samples, although comparisons of asexual cultures remain valuable (Lücking et al. 2020). Based on the work of Makonde et al. 2013, it is likely that other Termitomyces species exist where the only practical identification markers will be molecular.

Using the 3\% ITS similarity threshold, we find one lineage (group 1 sensu Vesala et al. 2017) that is distributed across Africa, with differing hosts depending on geography, but always within Macrotermes. These differences of termite symbionts combined with geographic isolation 
may represent barriers to gene flow. Further study should show if geographically separated populations of $T$. cryptogamus including populations associated with other Macrotermes species than $M$. natalensis all form a single biological species.

While comparisons of ITS sequences from T. cryptogamus sp. nov. and T. schimperi are not currently available, we feel these represent at least two species for two reasons. Firstly, the fact that we are unable to successfully amplify the ITS regions using the ITS1F and ITS4 primers, while we are able to amplify the LSU sequence indicates that there are likely mutations in the primer binding site, which are not found in $T$. cryptogamus sp. nov. for which ITS sequences are readily amplified. This indicates that $T$. schimperi likely has fixed substitutions not shared with $T$. cryptogamus sp. nov. Secondly, the relationships between the LSU sequences generated (Figure 3 ) from $T$. schimperi and $T$. cryptogamus sp. nov. show significant genetic difference, although the backbone nodes of the phylogeny do not receive statistical support of bootstrap values greater than 70 . Additionally, the recovery of two clades of $T$. schimperi indicates that $T$. schimperi is potentially paraphyletic and deserves further study. 


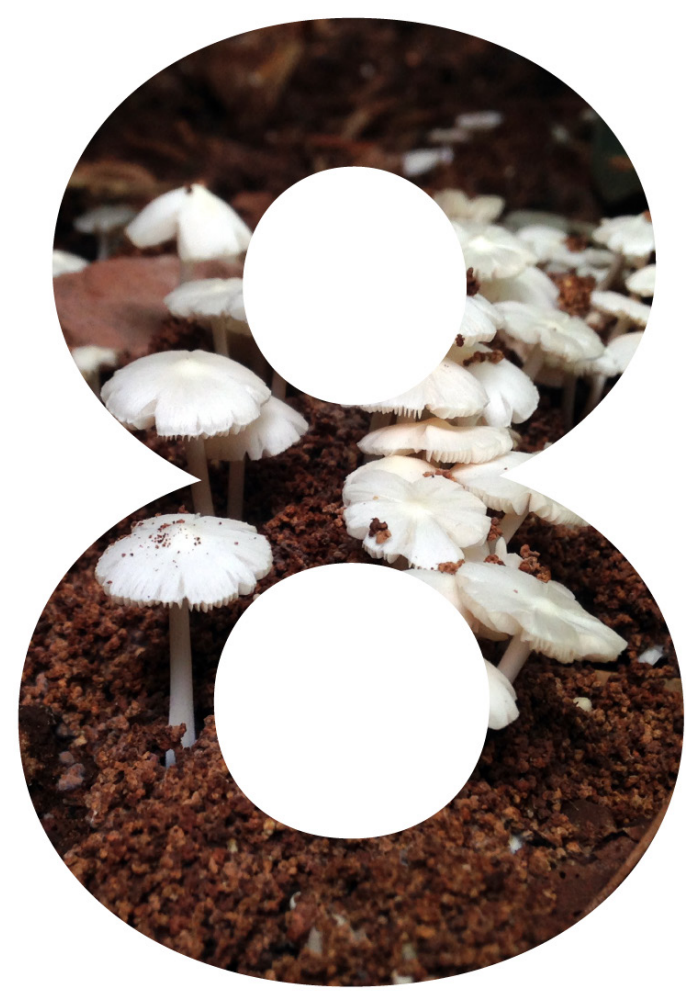

General discussion 
Mutualistic symbioses have been crucial for the evolution of life on earth and characterize major evolutionary transitions in evolution (Szathmáry and Maynard-Smith 1995, Maynard-Smith and Szathmáry 1997). For example, symbiogenesis may explain the evolution of eukaryotic cells from prokaryotic ancestors, as it suggests that cell organelles such as mitochondria and chloroplasts are derived from endosymbiotic prokaryotes (Sagan 1967). Mutualistic symbioses have increased biodiversity and have made it possible for organisms to explore new habitats. Fungi are an important example of this as many fungi are involved in associations with other organisms, and that way have expanded their own habitats as well as those of their associates. An important example of this are mycorrhizal fungi which had a critical role in the evolution and diversification of land plants (Wang and Qiu 2006). In this thesis, I focussed on a group of fungi, the basidiomycete genus Termitomyces, which lives in an obligate symbiosis with a subfamily of termites, the Macrotermitinae. The symbiosis between the fungusgrowing termites and their mutualistic fungi has a single origin in the rainforests of central Africa (Aanen and Eggleton 2005, Roberts et al. 2016). Since its origin many speciation events have taken place, both for the termites and for their fungal symbionts. The fungus-growing termites have expanded their range to cover almost the entire continent of Africa including the island of Madagascar and even parts of Asia. The single origin of fungiculture and the single successful domestication event of the fungus make this symbiosis unique among the main insectfungus mutualisms, since the other two have domesticated fungi multiple times independently. This uniqueness raises questions on origin of the symbiosis and differences between the other known insectfungus mutualisms.

In this thesis I focussed on the domestication and subsequent speciation of the termite-cultivated genus Termitomyces, thus approaching the symbiosis mainly from the fungal side. My first aim was to study the origin of the symbiosis. Using a phylogenomic approach I confirmed the monophyly of Termitomyces and identified Arthromyces as the sister group of Termitomyces. By studying the biology of Arthromyces and several other members of the termitomycetoid clade I reconstructed the biology of the ancestor of Termitomyces (Figure 1). I identified a set of shared traits between Termitomyces, its sister group Arthromyces and several other members of the termitomycetoid clade. These traits were most likely present in the common ancestor of Termitomyces and Arthromyces and so pre-dated the domestication of Termitomyces by termites. Those traits were: a rooting stipe of the mushrooms (a pseudorhiza), production of asexual spores (conidia) an 
insect-faecal pellet substrate, lack of clamp connections and a CAZyme profile with reduced capacity to degrade lignocellulose. I argued that this set of traits predisposed the common ancestor of Termitomyces to domestication (chapter 2). I studied the biology of the insectassociated fungus Blastosporella zonata, which belongs to the termitomycetoid clade, in more detail and found that the conidia produced by the fungus may be involved in local dispersal, while the wind-dispersed sexual spores typically disperse further. In addition, I also found evidence for occurrence of two cryptic biological species of Blastosporella (chapter 3). During the studies conducted in chapter 2 I discovered six species new to science within the termitomycetoid clade, which I describe in chapter 4.

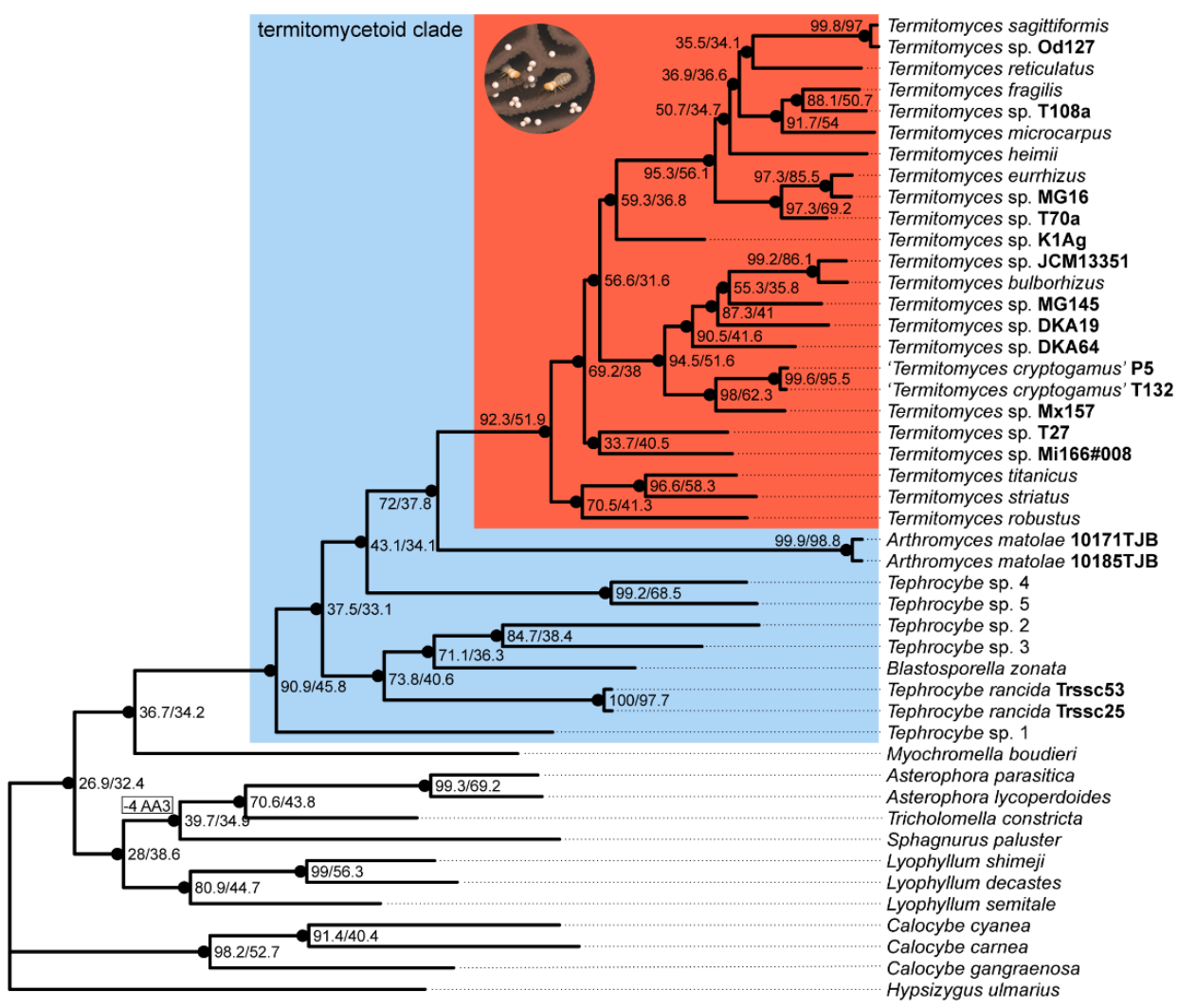

Figure 1 | Maximum-likelihood tree based on 1131 core nuclear (BUSCO) genes. Numbers at the nodes represent gene concordance factor/site concordance factor, dots indicate 100\% bootstrap support. Termitomycetoid clade highlighted in blue, termite-associated species highlighted in red. 
Next, I shifted my focus towards subsequent evolution of the genus Termitomyces, attempting to identify potential causes for speciation events within the genus. In chapter 5 I mapped the currently known genetic diversity in Termitomyces and delineated species based on molecular data. I used an Automatic Barcode Gap Discovery approach (Puillandre et al. 2012) to sort sequences into hypothetical species based on the DNA barcode gap, which is the difference between interand intraspecific genetic distances within a group. In addition, I considered differences in biology within the genus and reconstructed factors involved in speciation, such as host specificity and geographical isolation. Uniparental vertical symbiont transmission has been hypothesized to be an important contributing factor for (co-)speciation as it enhances host-specificity. Although horizontal transmission is the main symbiont transmission mode in fungus-growing termites, uniparental vertical transmission has evolved twice independently; once in the species Macrotermes bellicosus where vertical transmission is restricted to male reproductives, and once in the species of the genus Microtermes in female reproductives (Johnson 1981, Nobre, Fernandes, et al. 2011). Species in the genus Microtermes are inconspicuous as they do not construct large epigeal mounds but live in extensive tunnel systems underground. In chapter $\mathbf{6}$ I investigated host specificity and potential co-evolution in South African populations of Microtermes and their fungal symbiont and also attempted to estimate the size of a colony. I found evidence that colonies of Microtermes species may cover large areas and that there is frequent exchange of fungal symbionts.

The current taxonomic practice in mushroom-forming basidiomycetes, which is almost exclusively based on mushroom morphology, has caused issues in the taxonomy of Termitomyces since some species do not produce mushrooms in the wild. This leads to many species remaining undescribed, which hampers communication among scientists. Therefore, in chapter $\mathbf{7}$ I described the extensively studied species T. cryptogamus for which mushrooms have never been observed in the wild, based on a laboratory culture and DNA sequence data.

\section{The origin of the symbiosis}

Insect fungiculture has evolved independently several times (Mueller et al. 2005, Biedermann and Vega 2020). The three main systems are ambrosia beetles, attine ants, and the fungus-growing termites. Despite their independent evolution, several parallels or commonalities can be seen between the different systems. Examples of this are sociality in the 
insect host, the production of concentrated nutritional structures by the fungus and the evolution of vertical symbiont transmission. Because of the many commonalities, two main models for the evolution of insect fungiculture have been proposed; the "consumption-first" model and the "transmission-first" model (Mueller et al. 2001, Mueller et al. 2005). According to the consumption-first model an insect first consumes the fungus as part of its regular diet and later becomes adapted to cultivate it after which vertical transmission follows (Mueller et al. 2005). In contrast, according to the transmission-first model the insect is first vectoring the fungus and at some point, starts to derive nutrition from it, after which it starts with its cultivation. The consumption-first model has been suggested as the most likely scenario for the origin of the termite-fungus symbiosis because many wood-feeding termites show attraction to rotting wood (Batra and Batra 1979, Rouland-Lefèvre 2000). The evidence for this model is weak as it is based on observations on general termite behaviour and does not consider any knowledge on the biology of the sister groups of both the fungus-growing termites and their Termitomyces symbionts. With recent advances in knowledge on the evolutionary history of both termites and Termitomyces we are now able to identify key events, prior to the symbiosis, that eventually resulted in domestication.

\section{Nutritional symbioses in termites}

To understand the origin of fungiculture in termites we must know some key details on the biology of termites and especially about nutritional symbioses within this group. Termites have been classified as an order, Isoptera, separate from cockroaches (Blattodea). However, studies have shown that termites are nested within the Blattodea as they share a most recent common ancestor with the subsocial wood roach Cryptocercus, and so cockroaches do not form a monophyletic group. Therefore, the name Tutricablattae has been proposed for the group which includes Cryptocercus and all termites (Inward, Beccaloni, and Eggleton 2007, Lo et al. 2007, Evangelista et al. 2019). 


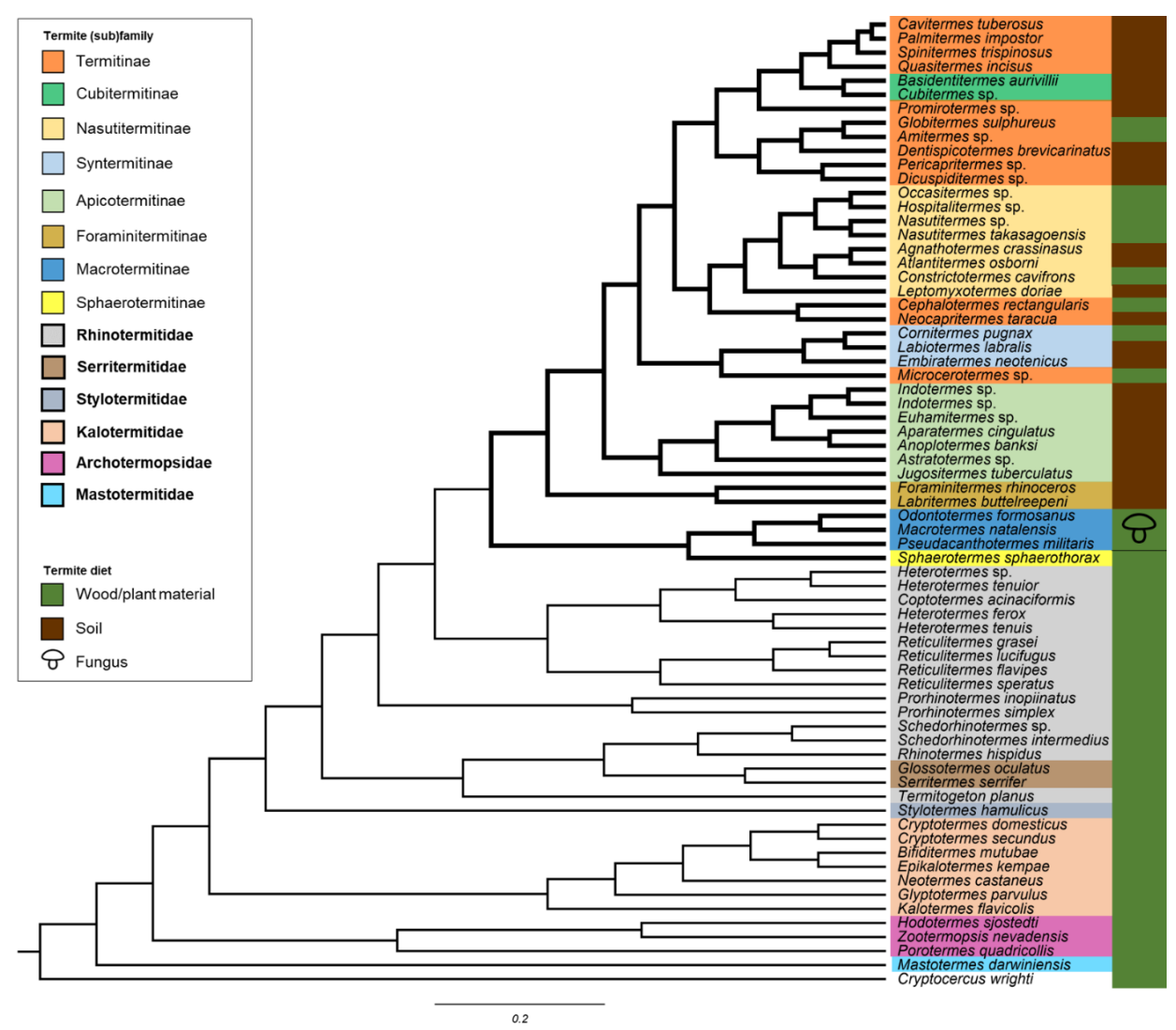

Figure 2 | Termite phylogeny with representatives of all the major extant termite families and their diet. Species in the Termitidae are indicated with thicker branches and within this family subfamilies are indicated. The Macrotermitinae cultivate fungi which are a secondary component of their diet, this is indicated with a mushroom symbol. The Sphaerotermitinae cultivate bacteria but it is not known whether the bacterial symbionts contribute directly to their diet. The outgroup is the wood roach Cryptocercus wrighti. Modified from Bucek et al. 2019

Most termites are plant feeders with transitions to bacteria-farming, fungus-farming and soil-feeding in the family Termitidae (Inward, Vogler, and Eggleton 2007, Chouvenc et al. 2021)(Figure 2). The lignocellulose in plant material is hard to digest because it is wellprotected against enzymatic breakdown, but termites are able to digest it with the assistance of nutritional symbionts (Cragg et al. 2015). All non-termitid termites and the sister group Cryptocercus carry flagellated protists in their gut, but these symbionts have been lost in the Termitidae. The loss of the symbiotic protists led to a diversification in feeding habits including bacteria-farming in the Sphaerotermitinae, 
fungus-farming in the Macrotermitinae and soil-feeding in the other Termitidae with many reversals to wood-feeding (Inward, Vogler, and Eggleton 2007, Chouvenc et al. 2021). The Sphaerotermitinae and Macrotermitinae are the only examples where the nutritional symbiont has been partially externalized (Figure 3) as the other Termitidae maintained intestinal Bacteria and Archaea. Recent research on the origin of the termite-fungus symbiosis has addressed the termite side of the symbiosis and has identified key evolutionary transitions in termite evolution which could help explaining the evolution of termite fungiculture (Bourguignon et al. 2015, Bucek et al. 2019, Chouvenc et al. 2021). The fungus-growing termites represent the only case among the fungus-growing insects in which the symbiotic fungus was successfully domesticated once. This could indicate that the predomesticated fungal ancestor possessed some unique traits that made it more suitable for domestication than any other wood-degrading fungus. Despite this potentially interesting evolutionary history, the fungal side of the symbiosis has not received much scientific attention. Therefore, in this thesis I thoroughly investigated the evolutionary origin of the termite-cultivated fungus Termitomyces and aimed to identify potential traits of the ancestor of Termitomyces that made it more likely to be domesticated.

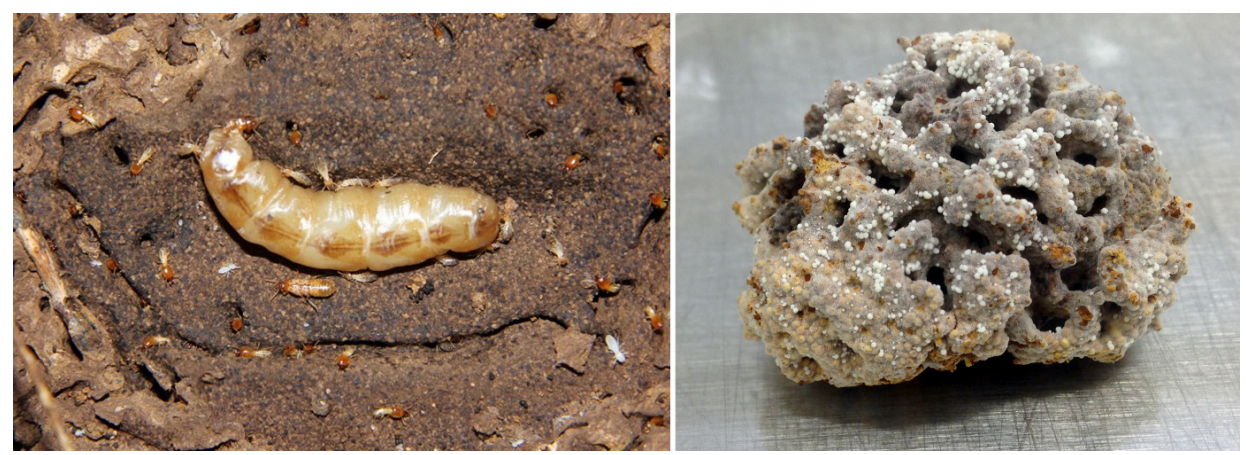

Figure 3 | Two examples of partially externalized digestion in the Termitidae. Picture on the left shows a queen of Sphaerotermes sphaerothorax on top of combs which harbour lignocellulolytic bacteria. The right picture shows a fungus comb of Microtermes sp., the white spheres are asexual fruiting bodies called 'nodules' which are consumed by the termites. Left picture by J. Šobotník.

Major transition in termite evolution: the loss of protozoan gut symbionts The key event that led to the diversification of the Termitidae was the loss of protist gut symbionts (Chouvenc et al. 2021). Interestingly, this loss of protist gut symbiont was followed by a diversification in feeding 
strategies such as fungus-farming, bacteria-farming and soil-feeding. There have been some difficulties reconstructing the phylogenetic placement of the early diverging bacteria-farming Sphaerotermitinae and the fungus-growing Macrotermitinae within the Termitidae. Multiple studies recovered the Macrotermitinae as the sister group to all the other Termitidae (Inward, Vogler, and Eggleton 2007, Bourguignon et al. 2015, Bourguignon et al. 2017). However, the most recent study on termite phylogenetics using transcriptome data found that Sphaerotermitinae is the sister group of Macrotermitinae, which together form a sister clade of all other Termitidae (Bucek et al. 2019)(Figure 4A). The phylogenetic placement for these early diverging groups is crucial as it has implications for the most parsimonious reconstruction of the biology of the common ancestor. The presumed Macro-Sphaerotermitinae sister-group relationship implies that the most parsimonious reconstruction is that the common ancestor of the two was a comb builder. It remains unclear whether this ancestral comb was sterile or inhabited by microbes. However, a sterile comb seems very unlikely as a comb constructed from faecal material seems a suitable environment for microbial organisms. Alternatively, the ancestral comb could have been inhabited by bacteria or fungi as both scenarios are equally parsimonious (replacement of bacteria by a fungus or fungi or vice versa).

In a review Chouvenc et al. pose two equally parsimonious scenarios on how the protist symbionts could have been lost in the Termitidae (Chouvenc et al. 2021)(Figure 4B,4C). The first scenario assumes that partially externalized digestion evolved in the common ancestor of the Termitidae and was followed by the evolution of soil feeding and reinternalization of the digestion after the split of the MacrotermitinaeSphaerotermitinae clade (Figure 4B). Some species in the sister group of the Termitidae already use woody faecal material as nest material, an example of this are the carton nests made by termites in the genus Coptotermes (Wood 1988, Eggleton 2006, Chouvenc, Efstathion, et al. 2013). The carton nests are used for regulating humidity and also offer resistance against entomopathogens by hosting Actinobacteria which produce antimicrobial compounds (Wood 1988, Chouvenc, Efstathion, et al. 2013, Chouvenc et al. 2018). The carton nest may have served as the basis for externalized digestion (Bignell 2006, Eggleton 2006, Aanen and Eggleton 2017). Since the function of the protist symbionts had been replaced by a form of partially externalized digestion they may have been lost over time. Later, different lignocellulolytic gut symbionts may have been reacquired as a new niche had opened up in the gut. A consequence of this may have been the transition to soil 
feeding in the non-externally digesting Termitidae. The second hypothesis assumes an early transition to soil-feeding in the ancestor of the Termitidae, which deprived the gut protozoa of cellulose eventually leading to a complete loss (Bucek et al. 2019)(Figure 4C). From their ancestral-state reconstruction Bucek et al. do not find strong evidence to either accept or reject this hypothesis. According to this second scenario, the loss of protozoa and transition to soil-feeding was followed by a quick reversal to wood-feeding and partially externalized digestion in the Macro-Sphaerotermitinae clade (Chouvenc et al. 2021).

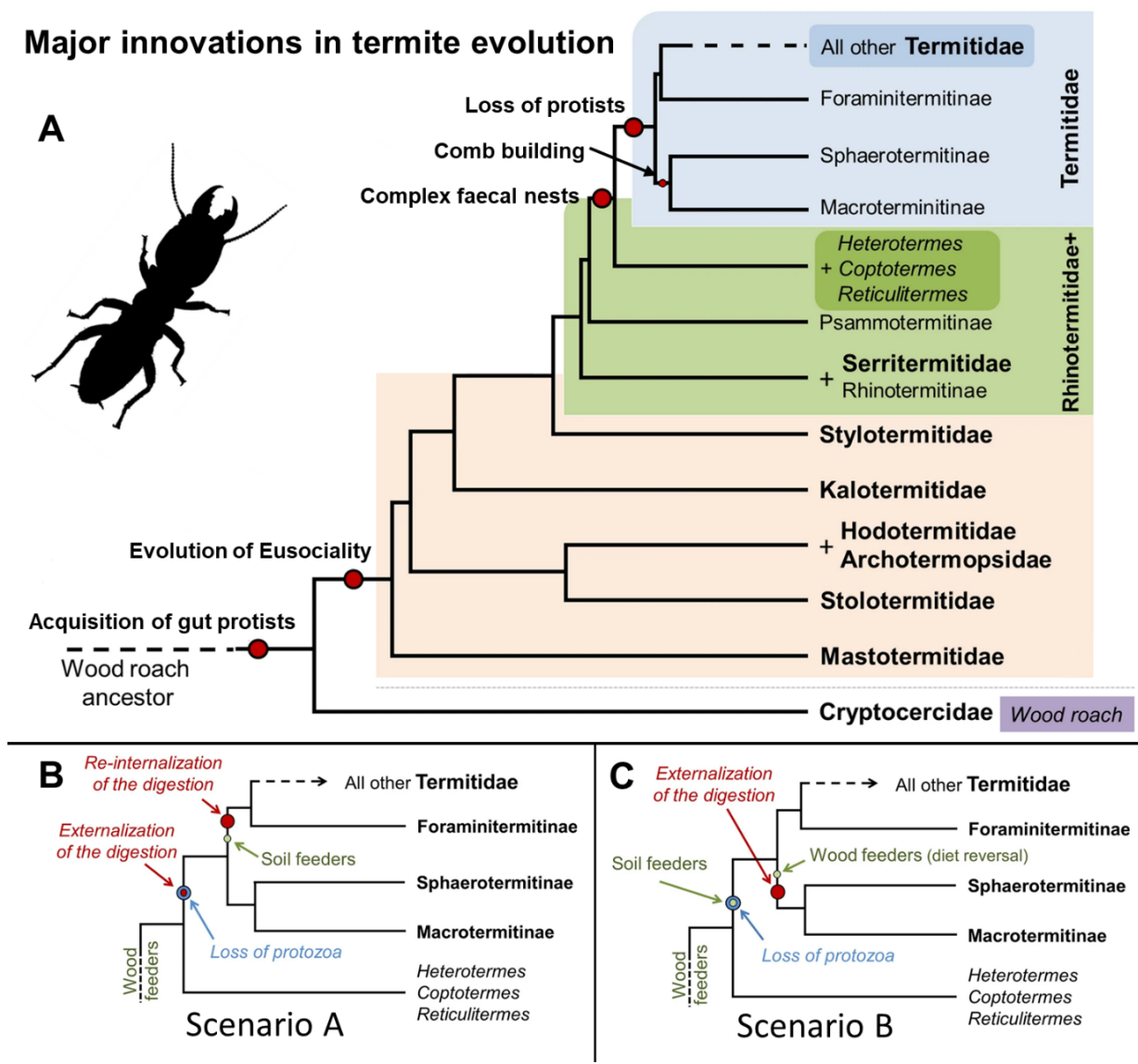

Figure 4 | Panel A showing a simplified phylogeny according to Bucek et al. 2019, relevant major evolutionary innovations are indicated on the tree with red circles. Panels B and C show two equally parsimonious scenarios explaining the loss of gut protozoa and subsequent evolution of soil-feeding and partially externalized digestion. All sub figures are modified from Chouvenc et al. 2021, in panel A the origin of comb building was added, the evolution of a frontal gland and adaptation to limited resources were removed. 
Partially externalized digestion and fungal domestication

Research on major transitions in termite evolution has received significant attention in the past two decades, which also has improved our understanding of the transition to fungiculture. Previous phylogenetic studies on the Lyophyllaceae did not show an insect relationship in species closely related to Termitomyces (Hofstetter et al. 2014, Bellanger et al. 2015). This has led researchers to assume that the ancestor of Termitomyces was a saprotrophic fungus. According to this view, the symbiosis might have originated via a consumption-first route in which the fungus was part of the regular diet of the ancestor of the fungus-growing termites (Rouland-Lefèvre 2000). This seemed reasonable as many termites feed on fungus-infested wood which also increases their survival (Viana et al. 2018).

In my thesis I show that Arthromyces is the sister group of Termitomyces and that Arthromyces is insect-associated as it grows on aggregated faecal pellets of an insect. I also show that the enzymatic machinery to break down carbohydrates is more similar between Arthromyces and Termitomyces than between Arthromyces and other members of the termitomycetoid clade. This implies that the ancestor of Termitomyces was not a common saprophyte but rather a fungus that was already specialized on insect faeces. As comb-building was most likely already present in the ancestor of the fungus-growing termites the ancestral comb may have provided a suitable niche. If we assume that the comb took over the role of gut protozoa it is likely that the ancestral comb was already inhabited by cellulolytic microorganisms. It has also been shown that in Coptotermes abandoned carton nests or parts of the nest which are abandoned can be colonized by saprophytic basidiomycetes such as Leucocoprinus (Chouvenc, Bardunias, et al. 2013). This indicates that the ancestral comb was an attractive substrate for microorganisms. It also means that the ancestor of Termitomyces had to outcompete cellulolytic microorganisms occupying the ancestral comb in addition to soil microorganisms, including other fungi, which could potentially occupy the same niche.

Major evolutionary transitions towards obligate fungiculture in termites The evolution of life on earth has seen a number of major transitions (Maynard-Smith and Szathmáry 1997). Some of these have happened multiple times independently across the tree of life, such as the transition from single cells to multicellularity and the origin of sociality (Szathmáry 2015). Other extant representatives of major transitions have a unique origin and are often referred to as 'singularities' (De Duve 2005). Examples of this are the transition from abiotic chemistry to living 
organisms and the transition from prokaryotic to eukaryotic cells (Maynard-Smith and Szathmáry 1997).

In his book on singularities, Cristian de Duve gives seven potential mechanisms which could account for these singularities, which I will briefly explain (De Duve 2005). The first mechanism is "deterministic necessity" which basically means things could not have been otherwise restricted by the laws of nature. This mainly applies to physical and chemical phenomena. The next three mechanisms all involve some sort of bottleneck. Probably the most well-known is the "selective bottleneck" imposed by natural selection. While a selective bottleneck is imposed by some external factor, a "restrictive bottleneck" is imposed by internal constraints, such as an existing body plan. These two types of bottlenecks often occur simultaneously. In some cases, a single lineage can emerge without a selective pressure, in this case we speak of a "pseudo-bottleneck". Mechanism five and six involve chance; in the case of "fantastic luck", an event can be so improbable that it most likely will not happen or in the case of a "frozen accident" there are multiple possibilities, but once a certain path is taken there is no way back. This can also mean that a transition may not be unlikely to happen but once a niche is occupied, a second transition is not possible anymore. Mechanism seven is "intelligent design" which I will not consider as such an explanation would involve intervention from outside the natural world and thus is not scientific.

Although both fungiculture and fungal domestication have analogous origins in other groups within the animal kingdom, and so are not singularities in the strict sense, fungiculture in termites and fungal domestication in the Lyophyllaceae are singular events. The singular domestication of Termitomyces by termites is unique among the major fungus-growing insect groups, since attine ants and ambrosia beetles have independently domesticated multiple fungal symbionts. The domestication of Termitomyces may not have been an improbable event considering the degree of 'preadaptation' in both the ancestor of fungus-growing termites (comb building) and the ancestor of Termitomyces (an insect-faecal association, a pseudorhiza, conidial production, a CAZyme profile with a reduced potential to degrade plant-cell wall components and a lack of clamp connections). However, the group of potential symbionts may have been extremely limited as the ancestral comb would have been a highly selective environment, which would have given a fungus with a termite-domestication syndrome a significant competitive advantage over other wooddegrading fungi. It is also unlikely that the domestication of Termitomyces led to complete niche occupation as bacteria-farming (in 
the case of the Sphaerotermitinae) represents a viable alternative for degrading plant material in the comb and a successful secondary fungal invasion in this group has not been observed.

The split between the Macrotermitinae and Sphaerotermitinae has been estimated to have occurred about 50 million years ago, while all extant species of Termitomyces descent from a common ancestor estimated about 20 million years later (Nobre, Kone, et al. 2011, Roberts et al. 2016, Bucek et al. 2019, Chouvenc et al. 2021). This means that different scenarios are possible, for example, a domestication event or multiple events between 50 and 30 million years ago with a severe bottleneck of the symbiont population around 30 million years ago, during which only a single lineage survived. There are some indications for an early bottleneck around 30 million years ago as this time period roughly coincides with an Eocene-Oligocene extinction event, caused by changes in climate, about 34 million years ago (Ivany, Patterson, and Lohmann 2000).

A single domestication event in a system with horizontal symbiont transmission and independent reproduction of the symbiont is rather paradoxical due to possible genetic exchange with free-living populations and the need to reobtain the symbiont from the environment, which may reduce host-symbiont fidelity. Since the symbiont needs to be obtained from the environment there is also the possibility to domesticate more than one symbiont. Uniparental vertical transmission is believed to greatly enhance host-symbiont fidelity and thus would enhance domestication (Frank 1997, Law and Dieckmann 1998, Herre et al. 1999). This may be one of the reasons why it independently evolved in all three fungus-growing insect systems (Francke-Grosmann 1967, Mueller, Rehner, and Schultz 1998, Korb and Aanen 2003). However, in fungus-growing termites, horizontal transmission has been maintained as the dominant mode of symbiont transmission (Korb and Aanen 2003). The fungus-growing termites represent the only case in which all symbionts are able to sexually reproduce but are also fully domesticated (meaning they do not occur free-living). Although the majority of fungal cultivars of attine ants still reproduce sexually, the cultivars of the so-called 'higher ants' are fully domesticated and reproduce primarily asexual and sexual reproduction is rarely reported under natural conditions (Mueller, Rehner, and Schultz 1998, Mueller 2002). The majority of fungi cultivated by ambrosia beetles have asexual reproduction although sexual stages have been reported in some symbionts (Farrell et al. 2001, van de Peppel, Aanen, and Biedermann 2018). As both asexual reproduction of the symbiont and uniparental vertical transmission create a 
reproductive barrier between symbiotic and free-living populations, a different type of barrier may have occurred in the symbiont population of fungus-growing termites. Alternatively, the Eocene-Oligocene extinction may have negatively affected the free-living members of the symbiont population, increasing the survival of fungi in the buffered conditions of the termite nests instead of the outside world, which eventually may have rendered the free-living population extinct. This dependence of the symbiont on the termite host would reduce the necessity of vertical transmission for the termites and therefore sexual reproduction could be maintained. Following this scenario, a single lineage may have survived which became domesticated or a subsequent additional bottleneck may have driven other independently domesticated lineages to extinction.

\section{The traits predisposing fungi to domestication by termites}

In chapter $\mathbf{2}$ I identified a set of traits which was most likely present in the ancestor of Termitomyces which I hypothesised predisposed it towards domestication. These traits are: an insect-faecal association, the production of conidia, the presence of a pseudorhiza (rooting stipe), a CAZyme profile with a reduced potential to degrade plant-cell wall components, the absence of clamp connections and a suitable carbohydrate-degrading enzymatic profile. All these traits are present in both Termitomyces and its sister group Arthromyces, strongly indicating that these traits were present in the common ancestor as well. Furthermore, various of these traits are found scattered in the termitomycetoid clade.

\section{Production of conidia}

Asexual sporulation is observed in a number of species in the Agaricomycetes, but the production of conidia on the mushroom is relatively rare (Kües et al. 2016). Different types of asexual spores (conidia) exist and they can be broadly placed in three categories: arthroconidia, blastoconidia, and chlamydospores. Blastoconidia are formed by budding from a weakened spot in the hyphal cell while arthroconidia are dissected from terminal hyphae. Chlamydospores are thick-walled and contain reserves such as lipids, glycogen and protein and are formed by condensation of the cytoplasm (Clémençon 1997). Because of these properties their main function is survival rather than dispersal. Species producing any of these three types of asexual spores are found in the Lyophyllaceae. Chlamydospores are formed by members of the genus Asterophora which parasitize on other basidiomycetes (Buller 1924). I found that most species in the 
termitomycetoid clade produce arthroconidia and that $B$. zonata produces both blastoconidia and arthroconidia. I hypothesized that the production of conidia in most members of the termitomycetoid clade is an important element in the lifecycle of these fungi. In the case of Termitomyces the function of the conidia is well-studied. The nodules, which produce the conidia, provide various nutrients and enzymes and conidia are used for re-inoculation of the combs (Leuthold, Badertscher, and Imboden 1989, Nobre and Aanen 2012). In chapter 3 we found signatures of clonality within localities but not between localities, indicating that conidia are used for local dispersal and that basidiospores serve long-range dispersal. Both B. zonata and species in the genus Arthromyces are growing on subterranean aggregated faecal pellets, a relatively homogeneous, but patchily distributed and probably seasonal substrate. The ability to rapidly colonize this substrate provides a competitive advantage over other fungi present in the soil. Conidia could facilitate rapid colonization as a single dikaryotic spore could colonize the substrate and fulfil the entire life cycle, without the need for two compatible basidiospores to colonize the substrate and mate to establish a secondary mycelium. As the conidia produced by Arthromyces and Blastosporella are relatively thick-walled they may also be able to survive in the soil when substrate is unavailable, similar to the thick-walled chlamydospores produced by mycoparasites in the genera Asterophora and Squamanita (Redhead et al. 1994).

The loss of clamps connections

In basidiomycetes mating occurs when two compatible homokaryons (mycelia with one type of haploid nucleus) meet and reciprocally donate a nucleus. After mating, the secondary mycelium or heterokaryon that is formed contains two different types of nuclei. In many species of basidiomycetes clamp connections are formed on the hyphae to maintain dikaryotic cells with one of each type of nucleus (Casselton and Olesnicky 1998). Species in the genus Termitomyces are an exception to this general pattern as they lack clamp connections and have variable numbers of up to ten nuclei per cell (De Fine Licht, Andersen, and Aanen 2005, Nobre et al. 2014). Interestingly, multinucleate cells in fungi, polyploidy or increased genome copy numbers, are observed in the symbionts in a range of mutualisms. Some examples of this are the multinucleate fungal cultivars of fungus-growing ants (Kooij et al. 2015), the multinucleate and multigenomic fungi forming arbuscular mycorrhizae with plants (Kuhn, Hijri, and Sanders 2001), the polyploid endosymbiotic plastids in plant cells and mitochondria in eukaryotic cells (Bendich 1987) and most of the crops domesticated by humans 
(Bretagnolle and Thompson 1995). A biological explanation for this striking pattern is lacking, but in plants polyploidy is often correlated with an increased size of plant organs such as fruits, this is often referred to as the 'gigas' effect (Sattler, Carvalho, and Clarindo 2016). A similar effect could be present in the symbiotic fungi of fungus-growing ants and fungus-growing termites as multinucleate cells are correlated with the production of gonglylidia or nodules.

The lack of clamp connections in the Lyophyllaceae is not restricted to the genus Termitomyces; also species in the genus Arthromyces (Baroni et al. 2007) and Gerhardtia (Vizzini et al. 2015) lack clamp connections. We found four additional species in the termitomycetoid clade to be clampless; the two species which make up the genus Praearthromyces and Nigrocarnea radicata (Tephrocybe sp2.) and Phaeotephrocybe leucophylla (Tephrocybe sp3.). This indicates three potentially independent losses of clamp connections in the Lyophyllaceae, one in the genus Gerhardtia, one in the common ancestor of $N$. radicata and $P$. leucophylla., and one in the clade containing the genera Praearthromyces, Arthromyces and Termitomyces. Since the loss of clamp connections is present in the genus Termitomyces but also in the sister genus Arthromyces as well as other members of the Lyophyllaceae the most parsimonious reconstruction is that this trait is also present prior to domestication. It is unclear whether a correlation exists between the absence of clamps and multinucleate cells as we did not determine the number of nuclei per cell in lyophylloid species other than Termitomyces.

The evolution of a pseudorhiza

A rooting stipe or pseudorhiza is a subterranean tapering root-like part of the stipe which connects the basidiocarp to a buried nutrient substrate. The pseudorhiza often has short lateral extending bundles of hyphae which attach to leaves or other objects in the soil. The major function of the pseudorhiza is to push the immature basidiocarp to the soil surface and to transport any nutrients necessary for further development of the basidiocarp (Buller 1934). A pseudorhiza has evolved independently several times in the Agaricomycetes, some examples I will list below. I will briefly discuss the evolution of the pseudorhiza in the Lyophyllaceae and then focus on the termitomycetoid clade.

The most common example of a buried nutrient substrate is buried wood in the case of Caulorhiza umbonata (Lennox 1979), Hypholoma radicosum (Parker 1933), some species of the genus Mycena (Smith 1947), the genus Phaeocollybia (Redhead and Malloch 
1986), and pseudorhiza-forming species of the genus Psathyrella (Van Waveren 1985) and Xerula radicata (Campbell 1938). Species of the genus Strobilurus usually grow on pinecones and produce a pseudorhiza in case the cone is buried (Singer 1962, Qin et al. 2018). This example shows that the trait can be plastic, only induced when needed. Various species in the genus Hebeloma are also known to produce a pseudorhiza, for example: $H$. luchuense (Fukiharu and Hongo 1995), H. radicosoides (Sagara et al. 2000a), H. radicosum and H. spoliatum (Sagara et al. 1981). Japanese collections of H. radicosum have been demonstrated to be molecularly distinct from European collections and have therefore been described as a new species: $H$. sagarae (Eberhardt et al. 2020). H. sagarae is usually associated with mole latrines where it also forms ectomycorrhizas with various tree species (Maeno et al. 2016, Eberhardt et al. 2020). Both H. sagarae and $H$. spoliatum have been associated with the latrines of the Japanese shrew mole, Urotrichus talpoides (Sagara et al. 1981). In addition, the Japanese species $H$. sagarae has been found associated to shrew (genus Sorex) latrines (Sagara, Ooyama, and Koyama 2008). Two species, $H$. radicosoides (initially misidentified as $H$. radicosum) and $H$. spoliatum have also been found on the abandoned nests of the wasp Vespula flaviceps lewisii where they grow on faecal pellets (Sagara et al. 1985). The European $H$. radicosum has been associated with latrines of the wood mouse (genus Apodemus)(Sagara, Senn-Irlet, and Marstad 2006). While $H$. radicosoides is part of a chemoecological group known as ammonia fungi, a group of fungi which fruit in response to a suddenly high concentration of ammonia or other nitrogenous material (Sagara 1975), H. sagarae does not respond to high concentrations of ammonia and may have developed a different association with the mammal latrines. A "habitat-cleaning symbiosis" has been proposed, which suggests that $H$. sagarae and its host tree produce ectomycorrhizas in mole latrines which remove compounds from the mole excreta to keep the nest clean (Sagara 1995, Sagara and Fukasawa 2014). All known species in the genus Termitomyces produce a rooting stipe except $T$. microcarpus (Heim 1977). Analogous to the unrelated species mentioned above, Termitomyces grows on a subterranean nutrient substrate, the fungus comb. Fruiting is initiated by primordia which are formed directly on the fungus comb (Heim 1977, Vreeburg et al. 2020).

The production of a pseudorhiza may be a rather plastic trait determined by the actual location and depth of the nutrient substrate. For example, M. galericulata is often encountered growing on nonburied tree stumps; however, when growing on buried wood it develops a pseudorhiza (Buller 1934, Smith 1947). There is also 
variability in this trait in the lyophylloid species Tricholomella constricta as it produces a short pseudorhiza in some cases (Kalamees 1992). The exact nutrient substrate for this species is not known, but fruiting in this species may be induced by urine (Sagara 1975), which in some cases might trigger hypogeous fruiting which creates the need for a pseudorhiza. The fact that $T$. microcarpus does not produce a pseudorhiza may not represent an actual loss of this trait but rather the lack of a phenotypic response due to epigeous fruiting, as this species fruits on parts of fungus comb with primordia which are expelled from the nest by the host termite (Heim 1977). When fungus-comb fragments with primordia of the species $T$. cryptogamus are incubated in the laboratory, the basidiocarps that are produced have elongated stipes but without a recognizable pseudorhiza (Vreeburg et al. 2020).

Within the Lyophyllaceae, the ability to produce a pseudorhiza is probably rather common: Myochromella inolens may produce a short pseudorhiza in some cases, Sagaranella tylicolor, which in some cases produces a pseudorhiza connected to black sclerotia (Knudsen and Vesterholt 2008), the previously mentioned T. constricta produces a small pseudorhiza in some cases (Kalamees 1992) and the species Gerhardtia pseudosaponacea also produces a small pseudorhiza (Cooper 2014). As mentioned previously the trait may show some degree of phenotypic plasticity or could evolve rather quickly. It could be that all or most species within the Lyophyllaceae can produce the pseudorhiza but only in some cases receive the right environmental trigger to actually produce it.

\section{The transition towards an insect-faecal nutrient substrate}

The sister group of the termitomycetoid clade, Myochromella, does not grow on a buried nutrient substrate and is most likely saprotrophic (Hofstetter et al. 2014). This is also reflected by the CAZyme profile of M. boudieri (Figure 5) which is similar to the CAZyme profile of Lyophyllum decastes which is a saprotroph and also able to form ectomycorrhizal associations (Pera and Alvarez 1995).

All species in the termitomycetoid clade produce a pseudorhiza. This suggests that a transition occurred from a saprotrophic lifestyle towards a lifestyle on a buried nutrient substrate in the ancestor of termitomycetoid clade. Whether this substrate consisted of faecal pellets produced by an insect or something different remains unclear as the nutrient substrate of Australocybe olivacea (Tephrocybe sp1.) as well as four other key species in the termitomycetoid clade has not been identified. 


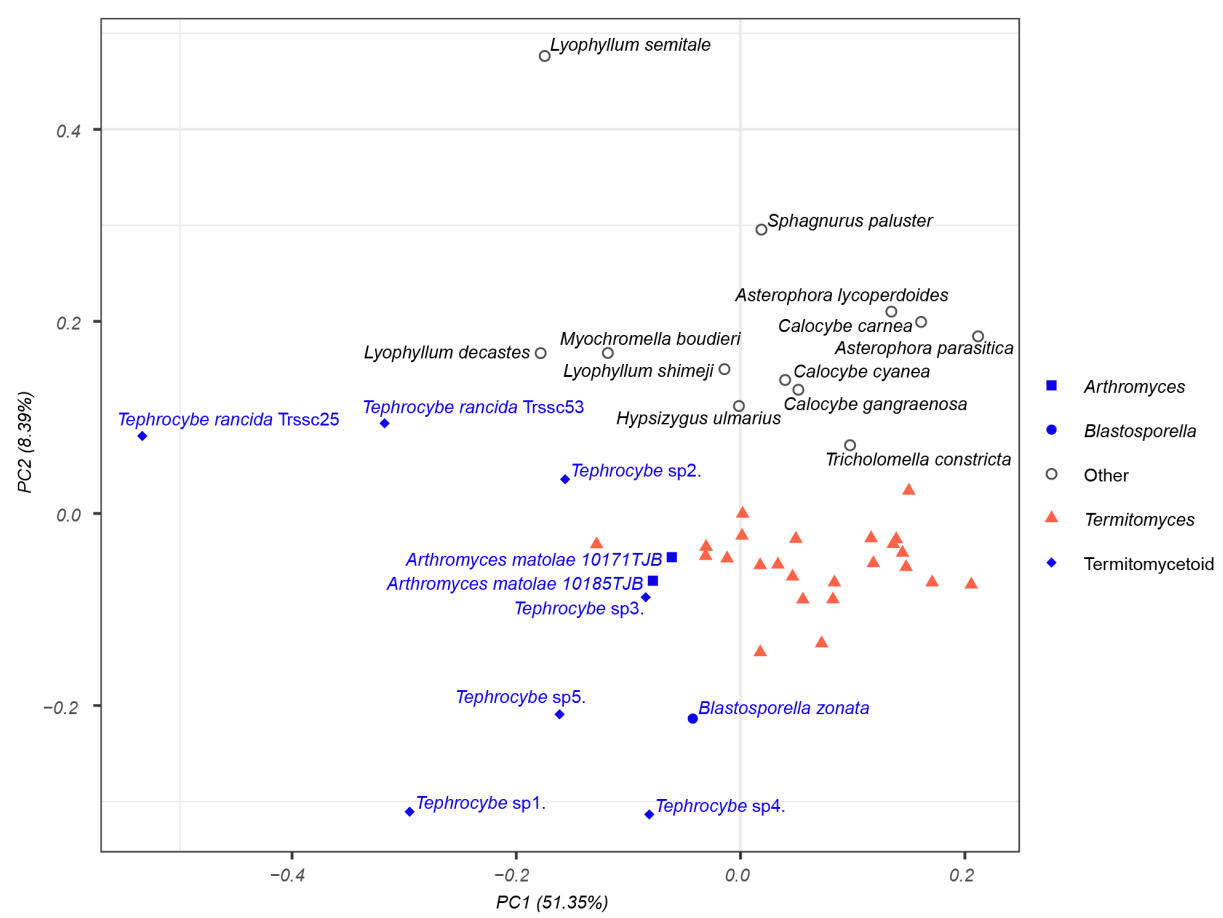

Figure $\mathbf{5}$ | Principal component analysis of 119 CAZyme subfamilies, numbers in parentheses indicate the percentage of variance explained by a principal component.

The transition from a saprotrophic lifestyle towards an insect-faecal association seems rather drastic as this transition may require several specific additional adaptations, such as the production of conidia or a CAZyme profile with a reduced potential to degrade plant-cell wall components. Therefore, an initial transition in the ancestor of termitomycetoid clade towards a non-faecal buried nutrient substrate, such as buried wood, seems logical (Figure 6A,B,C). Since we only have evidence for the presence of an insect-faecal association in the genera Arthromyces, Blastosporella and Termitomyces and the absence of such an association in the species $T$. rancida, a plausible scenario is an initial transition to a buried nutrient substrate in the ancestor of the termitomycetoid clade followed by two independent transitions to an insect-faecal nutrient substrate in the ancestor of Arthromyces and Termitomyces and in the species B. zonata (Figure6A). Assuming the genera Praearthromyces, Nigrocarnea and Phaeotephrocybe are also insect-faecal associated earlier transitions in the ancestors of the Praearthromyces-Arthromyces-Termitomyces clade and the Blastosporella-Nigrocarnea-Phaeotephrocybe clade are also possible 
(Figure 6B). Alternatively, again assuming insect-faecal associations in most species in the termitomycetoid clade, after the initial transition towards a buried nutrient substrate in the ancestor of the termitomycetoid clade a single transition towards an insect-faecal nutrient substrate could have taken place after the split with Australocybe, with a single reversal towards the ancestral lifestyle in $T$. rancida (Figure 6C).

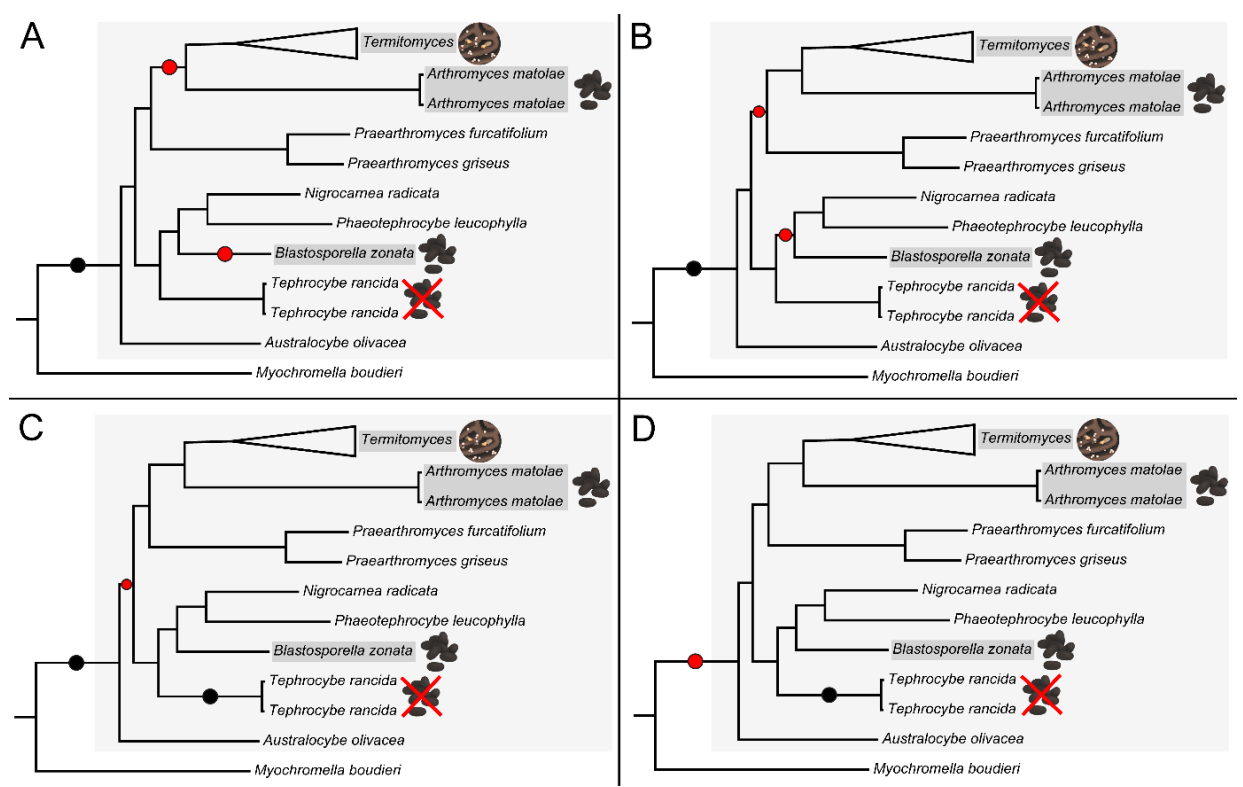

Figure 6 | Four scenarios that could explain the evolution of an insect-faecal association in the termitomycetoid clade. Black circles represent a transition to a buried nutrient substrate other than insect faeces, red circles represent a transition to an insect-faecal nutrient substrate. Insect-faecal associated species are highlighted in grey, T. rancida is not insect-faecal associated and the substrate for all other species has not been identified. An early transition towards a non-faecal buried nutrient substrate in the ancestor of the termitomycetoid clade followed by two independent transitions to insect faeces in the ancestor of Arthromyces and Termitomyces and B. zonata (A) or in the ancestors of the Praearthromyces-Arthromyces-Termitomyces and Blastosporella-Nigrocarnea-Phaeotephrocybe clades (B) or a single transition after the split of $A$. olivacea and subsequent loss in the T. rancida clade (C). Alternatively, an early transition towards an insect-faecal nutrient substrate in the ancestor of the termitomycetoid clade followed by a single loss in the $T$. rancida clade (D).

An early transition to an insect-faecal nutrient substrate in the ancestor of the termitomycetoid clade is also plausible and suggests that insectfaecal associations are the rule in the termitomycetoid clade and that $T$. 
rancida would represent the only reversal to a saprotrophic lifestyle (with a rudimentary pseudorhiza) or a transition to a buried nutrient substrate other than insect faeces (Figure 6D). In addition to the increase sharp increase in total CAZymes in T. rancida (chapter 2), the CAZyme profile of $T$. rancida also hints in this direction as it is closer to Lyophyllum, while the other termitomycetoid members are closer to the insect-faecal associated genera Termitomyces and Arthromyces (Figure $5)$.

Identifying the nutrient substrate of all species within the termitomycetoid clade would be crucial in reconstructing the transitions from a saprotrophic lifestyle towards an insect-faecal lifestyle and eventually a domesticated lifestyle.

\section{Parallels between human agriculture and termite fungiculture}

Domestication can be defined as an evolutionary interaction in which a novel dispersal mechanism is acquired by a producer species while its performance is controlled for the benefit, usually nutritional, of a consumer species (Milla et al. 2015). According to this definition, domestication does not have to be a conscious process and can therefore be applied to fungus-growing insects and various other systems, which allows us to make comparisons between human crop domestication and the fungal domestication by termites. I argue that many aspects in the evolution of the termite-fungus symbiosis are analogous to the domestication of crops by humans. Many of the crops domesticated by humans share several traits which have been subjected to artificial selection and set the crops apart from their wild progenitors, this set of traits has been referred to as a domestication syndrome (Harlan, de Wet, and Price 1973, Hammer 1984). Some of these traits are the loss of seed dispersal mechanisms, size increase of the harvestable organ (such as fruits or seeds), erect growth habit and the loss of photoperiod sensitivity (Meyer and Purugganan 2013). The Termitomyces symbionts of the fungus-growing termites also exhibits a particular set of traits (discussed above) which were likely already present in the wild progenitor of Termitomyces. The lifestyle of this wild progenitor was close to Termitomyces, as they both grow on insect faeces. This may be similar to the period following human crop domestication during which wild plants were cultivated by humans, this is called 'pre-domestication cultivation' (Abbo and Gopher 2020). The local dispersal of asexual, non-wind dispersed, fungal spores by an insect is analogous to the harvesting and sowing of seeds by humans in human agriculture. The loss of seed shattering which resulted in decreased natural seed dispersal is thought to have been a key trait 
favoured by selection under cultivation (Diamond and Ordunio 1999, Purugganan and Fuller 2009). The reduced natural dispersal caused by the loss of seed shattering caused plants to become dependent on humans for dispersal (Diamond 2002). This is remarkably similar to Termitomyces symbionts of the fungus-growing termites, which rely on the termite host for colonization of the substrate and their local dispersal (Leuthold, Badertscher, and Imboden 1989). As insect-faecal associations and the production of conidia are already present in the sister group of Termitomyces the dispersal of these conidia by an insect seems a crucial step in the domestication process of the ancestor of Termitomyces.

\section{Speciation in fungus-growing termites and Termitomyces symbionts}

Despite our findings that the ancestor of Termitomyces may have been predisposed to become domesticated there has only been one successfully domesticated lineage which made it to the current day. However, after the termites domesticated their fungus, multiple speciation events occurred for both the host termites and their fungus, eventually leading to the currently known diversity of about 330 species of fungus-growing termites (Eggleton et al. 2000) and 87 phylogenetic species (Box 1) of Termitomyces (chapter 5). The initial step in speciation probably took place shortly after fungal domestication, while the second step probably took place after migration out of the ancestral rainforest habitat to the drier savannahs (Aanen and Eggleton 2005, Nobre, Kone, et al. 2011, Roberts et al. 2016). After migration out of the ancestral habitat, at least four independent inter-continental migrations of the termites (Aanen and Eggleton 2005) and seven independent inter-continental migrations of Termitomyces symbionts have taken place (chapter 5). Since I could not find any evidence for genetic exchange between African and Asian populations of Termitomyces, I concluded that these populations are reproductively isolated. This reproductive isolation has resulted in speciation of Termitomyces as Asian species make up more than half of total number of species that I identified (chapter 5).

Speciation occurred much less frequently in the Sphaerotermitinae, the sister group of the fungus-growing termites, which is currently represented by just a single species, Sphaerotermes sphaerothorax (Bucek et al. 2019). The Sphaerotermitinae have evolved a similar form of complementary external digestion, but with lignocellulolytic bacteria instead of a fungus (Garnier-Sillam et al. 1989). This raises the question on what may have caused this difference in 
speciation between the Sphaerotermitinae and the Macrotermitinae. A truly fair comparison cannot be made as the exact symbionts of $S$. sphaerothorax have not been identified and could potentially be diverse among the same host species.

Nevertheless, the first factor that may play a role is the fact that wood-decay fungi are usually better lignin degraders than lignocellulolytic bacteria (Sigoillot et al. 2012). This may result in a more flexible diet for a fungus-grower than a bacteria-grower as the amount of lignin may become a limiting factor in the termite diet. More variation in the termite diet could potentially open up new niches for the symbiotic fungus, eventually leading to specialization and speciation. This may be the cause of the differences in nutritional preferences between different species of Termitomyces (da Costa et al. 2019). It also could have made the fungus-growing termites a stronger competitor over bacterial growers as they would be more efficient at digesting the same type of resources.

The Sphaerotermitinae are only found in central Africa (Šobotník and Dahlsjö 2017) and have not migrated out of their original range in contrast to the fungus-growing termites. The migration out of the humid rainforests by the fungus-growing termites may have required several adaptations in the regulation of humidity and temperature in the nest. This is probably reflected by the enormous diversity in mound architecture ranging from the subterranean gallery systems of species in the genus Microtermes to the tall epigeal chimney-like mounds of species in the genus Macrotermes (Uys 2002). Domestication of the fungal crop may be required as free-living populations of the fungus may not survive outside the native habitat. The bacteria grower $S$. sphaerothorax constructs subterranean nests (Grassé and Noirot 1948), this might have posed limitations on its capability to migrate to the drier savannahs but is probably not the most important factor. Of more importance seems to be the acquisition of bacterial symbionts by $S$. sphaerothorax. Knowledge on the composition of the bacterial population on the combs of S. sphaerothorax as well as its lifecycle and the acquisition of symbionts is lacking. In case the symbionts are not vertically transmitted but have to be obtained from the environment, this may have been another limitation, especially if the symbionts are restricted to a rainforest habitat. There is some analogy between the fungus-growing termites and the fungus-growing ants, as in the attine ants fungus-growing also evolved in the rainforests, but in south America (Mueller et al. 2001). However, fungal domestication by attine ants most likely occurred in a seasonally dry habitat, which may have favoured isolation of their cultivar (Branstetter et al. 2017). The evidence 
suggests that fungal domestication in fungus-growing termites happened prior to the migration out of the rainforests (Aanen and Eggleton 2005, Nobre, Kone, et al. 2011), so the migration out of the rainforests probably did not play a role in the domestication but may have been important for subsequent speciation events.

Another potential adaptation in fungus-growing termites, which may have resulted in speciation in Termitomyces is the evolution of uniparental vertical symbiont transmission. Uniparental vertical symbiont transmission evolved twice in the fungus-growing termites, in the female reproductives of the genus Microtermes and in the male reproductive of the species Macrotermes bellicosus (Johnson 1981, Nobre, Fernandes, et al. 2011). In chapter 5 and chapter 6 I showed that contrary to theory, uniparental vertical transmission has not resulted in tight host-symbiont relationships or co-evolutionary patterns as both $M$. bellicosus and species in the genus Microtermes cultivate several different species of Termitomyces, which are also cultivated by other species in the same genus or even different genera in the case of Microtermes. This implies that horizontal transmission occurs frequently for those species. Analogously, leaf-cutting ants have predominantly uniparental vertical transmission but occasional horizontal transmission has also been reported (Howe, Schiøtt, and Boomsma 2018).

The dietary composition, and thus the substrate for the fungus, may also play a role in symbiont selection by the host termite (da Costa et al. 2019). Therefore, shifts in dietary composition may have also promoted interaction specificity which could have resulted in speciation. However, specificity could also be the result rather than the cause of speciation. Not much is known about the diets of fungusgrowing termites and about mechanisms by which termites select their fungal symbiont. Identifying the mechanisms involved in symbiont selection may be valuable in understanding some of the factors which may underlie speciation events in the genus Termitomyces.

Box 1: Species concepts and species delimitation

To categorize life on earth, humans classify organisms into species. In order to delineate between different species a strong and coherent definition of a species is required. The definition of a species is called a species concept. One would expect that there is a single universal species concept; however, as many as 24 different species concepts have been reported (Mayden 1997). Probably the most well-known species concept is the biological species concept which states that: a 
species is an interbreeding group which is reproductively isolated from other such groups (Mayr 2000). A second species concept is the evolutionary species concept: a species is a lineage of ancestral descendant populations which maintains its identity from other such lineages and which has its own evolutionary tendencies and historical fate (Simpson 1951). A third, commonly used species concept is the phylogenetic species concept: a species is the smallest aggregation of populations (sexual) or lineages (asexual) diagnosable by a unique combination of character states in comparable individuals (Nixon and Wheeler 1990). The phenetic or morphological species concept defines a species as a set of organisms that look similar to each other and is distinct from other sets. Although still often used, this species concept has some problems, as it lacks a clear criterion and often conflicts with a phylogenetic species concept (chapter 4). The disagreement among biologists about the definition of a species results in problems when delimiting species as species boundaries are unclear (De Queiroz 2007). A unified of universal species concept would immediately solve any issues in species delimitation as there would be no disagreement on the boundaries and definition of a species (De Queiroz 2007).

Fungal taxonomy is traditionally largely based on morphological characters of sexual or asexual states, particularly of spores, fruiting structures and cell morphology (Talbot 1971). With the advent of DNA-sequencing techniques molecular evidence has become a major part of taxonomy and species delimitation. DNA-barcoding is an approach to identify species based on a short genetic marker, in fungi the nuclear ribosomal internal transcribed spacer (ITS) region (Schoch et al. 2012). Researchers have used an arbitrary cut-off value between 3-5\% ITS sequence divergence to delimit species (Raja et al. 2017), but this method has been criticized (Nilsson et al. 2008). A reliable approach to delimitate species may combine morphologybased, DNA sequence based approaches and the results of mating tests (Aanen et al. 2000, Lücking et al. 2020). This comparison between different species concepts may also shed light on the process whereby reproductive barriers arise by comparing the relationship between genetic divergence and reproductive isolation (Aanen et al. 2000).

\section{Taxonomic challenges in the genus Termitomyces}

Good taxonomy is important for clear communication in the biological sciences (Hibbett and Taylor 2013). Many of the commonly used species names of Termitomyces are from descriptions which predate 
the DNA sequencing era and are solely based on morphological characters (Heim 1942, 1951, 1977). With the incorporation of DNA sequences in fungal taxonomy, a phylogenetic species concept is more often used than a morphological species concept (Box 1). Morphologybased taxonomy of Termitomyces has resulted in a strong bias towards the description of species which (regularly) form mushrooms. This has kept a big part of the current diversity within Termitomyces out of the scope of taxonomy as fruiting events are extremely rare or perhaps even absent in some species (Johnson 1981, Darlington 1994, Korb and Aanen 2003, de Fine Licht, Boomsma, and Aanen 2006). Some of this cryptic diversity has been noted in molecular phylogenetic studies as well (Rouland-Lefevre et al. 2002, Froslev et al. 2003, Osiemo et al. 2010). The use of laboratory cultures for taxonomical purposes has been proposed (Makonde et al. 2013), although cultures have some diagnostic features, a culture alone is not sufficient to delineate species (Botha and Eicker 1991a, b, Tibuhwa, Kivaisi, and Magingo 2010, Tibuhwa 2012). The current nomenclatural code requires a physical specimen or illustration for typification (art. 8.1) and the specimen needs to consist of metabolically inactive material (art. 8.4)(Turland et al. 2018). For the publication of a valid name, these requirements exclude the use of either a DNA sequence or a living culture for typification, which hampers the description of species of Termitomyces that rarely produce mushrooms. A disconnect also arises as DNA sequences from old type specimens are not available and thus names cannot be connected to specimens of which only cultures are available. In chapter $\mathbf{5}$ I managed to sequence some of these old type specimens which helps in solving this issue. In chapter $\mathbf{5}$ I also demonstrated that morphological species may not always be congruent with phylogenetic species because of convergent evolution or cryptic phylogenetic species. In chapter $\mathbf{3}$ I showed the existence of two cryptic species in $B$. zonata, in this case biological species are congruent with phylogenetic species but not with morphological species. Another example of incongruency between morphological and phylogenetic species are Asian lineages of Termitomyces which are morphological similar or identical to their African counterparts, but belong to phylogenetically distinct clades. In chapter 7 I describe T. cryptogamus, a species without any recorded natural basidiocarps. I base my description on a combination of molecular, ecological, and cultural diagnostic features. As natural basidiocarps were lacking for this species we used a dried culture for typification. Our initial idea of using either the living culture or a DNA sequence as a type would result in an invalid name. The use of DNA sequences for typification has been suggested (Hawksworth et 
al. 2016), but is still controversial (Zamora et al. 2018). The use of DNA sequences for typification would allow scientists to describe valid species names with complete absence of any physical material of the species. This means that valid species names could be described from any environmental DNA-barcoding study for example. However, fungal taxonomy based on DNA barcodes would require a strong reference database with a sufficient taxonomic coverage, correctly identified and annotated sequences, and standardized translation of this data into species names. This, however, is often not the case (Nilsson et al. 2006).

\section{Future outlook}

To resolve the taxonomic challenges that I described above I would urge taxonomists to always include a DNA sequence or multiple sequences of the type collection, preferably ITS, with their description of a novel species. This is currently not required by the taxonomic code (Turland et al. 2018). In order to resolve the bias towards the description of species which regularly produce mushrooms I would urge researchers to publish species, of which only cultures are available, using the same approach as I did in chapter $\mathbf{7}$ with T. cryptogamus. The species hypotheses I generated in chapter $\mathbf{5}$ could serve as a framework for future taxonomists to identify whether their species is still undescribed or matches to something already described. As public culture collections are becoming more accessible to scientists, I strongly recommend taxonomists to submit a culture of their novel species to a public culture collection as well. Currently only dried cultures are allowed for typification, which is a shame, as they did not contain many diagnostic features and are therefore not very useful for taxonomical purposes. For future DNA they are also not very useful, as DNA will degrade over time. Storage space, time, and resources spent on maintaining collections of dried cultures could be better spent on maintaining collections of living cultures or improved curation of DNA sequence databases. Changing the code to allow living cultures stored in recognized public culture collections (perhaps in combination with DNA sequence data) for typification could be an important first step to improve the taxonomy in the genus Termitomyces and perhaps in other fungal groups as well. 


\section{References}

Aanen, D. K., H. H. de Fine Licht, A. J. Debets, N. A. Kerstes, R. F. Hoekstra, and J. J. Boomsma. 2009. "High symbiont relatedness stabilizes mutualistic cooperation in fungus-growing termites." Science 326 (5956): 1 103-6. doi: 10.1126/science.1173462.

Aanen, D. K., and P. Eggleton. 2005. "Fungus-growing termites originated in African rain forest." Current Biology 15 (9):851-5. doi: 10.1016/i.cub.2005.03.043.

Aanen, D. K., and P. Eggleton. 2017. "Symbiogenesis: Beyond the endosymbiosis theory?" Journal of theoretical biology 434:99-103. doi: 10.1016/i.jtbi.2017.08.001.

Aanen, D. K., P. Eggleton, C. Rouland-Lefevre, T. Guldberg-Froslev, S. Rosendahl, and J. J. Boomsma. 2002. "The evolution of fungus-growing termites and their mutualistic fungal symbionts." Proc Natl Acad Sci U S A 99 (23):1 4887-92. doi: 10.1073/pnas.222313099.

Aanen, D. K., and R. F. Hoekstra. 2007. "The evolution of obligate mutualism: if you can't beat 'em, join 'em." Trends Ecol Evol 22 (10):506-9. doi: 10.1016/j.tree.2007.08.007.

Aanen, D. K., T. W. Kuyper, T. H. Mes, and R. F. Hoekstra. 2000. "The evolution of reproductive isolation in the ectomycorrhizal Hebeloma crustuliniforme aggregate (Basidiomycetes) in northwestern Europe: a phylogenetic approach." Evolution 54 (4):1192-206. doi: $10.1111 / \mathrm{i} .0014-3820.2000 . t b 00554 . x$.

Aanen, D. K., V. I. Ros, H. H. de Fine Licht, J. Mitchell, Z. W. de Beer, B. Slippers, C. Rouland-Lefevre, and J. J. Boomsma. 2007. "Patterns of interaction specificity of fungus-growing termites and Termitomyces symbionts in South Africa." BMC Evol Biol 7:1 15. doi: 10.1186/1471. 2148-7-115.

Abarenkov, Kessy, R Henrik Nilsson, Karl-Henrik Larsson, lan J Alexander, Ursula Eberhardt, Susanne Erland, Klaus Høiland, Rasmus Kiøller, Ellen Larsson, and Taina Pennanen. 2010. "The UNITE database for molecular identification of fungi-recent updates and future perspectives." The New phytologist 186 (2):281-285.

Abbo, S., and A. Gopher. 2020. "Plant domestication in the Neolithic Near East: The humans-plants liaison." Quaternary Science Reviews 242:106412. doi: 10.1016/i.quascirev.2020.106412.

Adams, R. M., U. G. Mueller, A. K. Holloway, A. M. Green, and J. Narozniak. 2000. "Garden sharing and garden stealing in fungus-growing ants." Naturwissenschaften 87 (11):491-3. doi: $10.1007 / \mathrm{s} 001140050765$.

Alamouti, S. M., C. K. M. Tsui, and C. Brevil. 2009. "Multigene phylogeny of filamentous ambrosia fungi associated with ambrosia and bark beetles." Mycological Research 113 (8):822835. doi: 10.1016/i.mycres.2009.03.003.

Ané, C., B. Larget, D. A. Baum, S. D. Smith, and A. Rokas. 2007. "Bayesian estimation of concordance among gene trees." Molecular Biology and Evolution 24 (2):412-26. doi: $10.1093 / \mathrm{molbev} / \mathrm{msl} 170$.

Axelrod, R., and W. D. Hamilton. 1981. "The evolution of cooperation." Science 211 (4489):13906. doi: 10.1126 /science.7466396.

Baroni, T. J., A. E. Franco-Molano, D. J. Lodge, D. L. Lindner, E. Horak, and V. Hofstetter. 2007. "Arthromyces and Blastosporella, two new genera of conidia-producing lyophylloid agarics (Agaricales, Basidiomycota) from the neotropics." Mycological research 111 (5):572-580. doi: 10.1016/i.mycres.2007.03.007.

Baroni, T. J., N. W. Legon, R. Vilgalys, and D. J. Lodge. 1999. "Calocybe cyanea - a rare and beautiful agaric is discovered in Puerto Rico." Mycologist 13:7.

Bas, C. 1965. "The genus Squamanita." Persoonia 3 (3):331-359.

Batra, L. R. 1963. "Ecology of ambrosia fungi and their dissemination by beetles." Transactions of the Kansas Academy of Science 66 (2):213-236. doi: 10.2307/3626562.

Batra, L.R., and S.W.T. Batra. 1979. "Termite-fungus mutualism." Proceedings of the Second International Mycological Congress, Insect-Fungus Symbiosis. Nutrition, Mutualism and Commensalism, University of South Florida, Tampa, Florida.

Bellanger, J. M., P. A. Moreau, G. Corriol, A. Bidaud, R. Chalange, Z. Dudova, and F. Richard. 2015. "Plunging hands into the mushroom jar: a phylogenetic framework for Lyophyllaceae (Agaricales, Basidiomycota)." Genetica 143 (2):169-194. doi: 10.1007/s10709-0159823-8. 
Bendich, A. J. 1987. "Why do chloroplasts and mitochondria contain so many copies of their genome?" BioEssays 6 (6):279-282.

Berkeley, M. J., and C. E. Broome. 1871. "The Fungi of Ceylon." Journal of the Linnean Society of London, Botany 11 (56):537.

Biedermann, P. H., and M. Taborsky. 2011 . "Larval helpers and age polyethism in ambrosia beetles." Proceedings of the National Academy of Sciences 108 (41):17064-9. doi: 10.1073/pnas. 1107758108.

Biedermann, P. H. W., and F. E. Vega. 2020. "Ecology and Evolution of Insect-Fungus Mutualisms." Annual Review of Entomology 65:431-455. doi: 10.1146/annurev-ento-011019-024910.

Bignell, D. E. 2000. "Introduction to Symbiosis." In Termites: Evolution, Sociality, Symbioses, Ecology, edited by T. Abe, D. E. Bignell and M. Higashi, 189-208. Dordrecht: Springer Netherlands.

Bignell, David E. 2006. "Termites as soil engineers and soil processors." In Intestinal microorganisms of termites and other invertebrates, 183-220. Springer.

Bignell, David Edward, Yves Roisin, and Nathan Lo. 2010. Biology of termites: a modern synthesis: Springer Science \& Business Media.

Bingham, M.G. 2002. "Are species of termitomyces specific to their host termites?" Kirkia 18:77-82.

Botha, W. J., and A. Eicker. 1991 a. "Cultural studies on the genus Termitomyces in South Africa. I. Macro-and microscopic characters of basidiome context cultures." Mycological Research 95 (4):435-443.

Botha, W. J., and A. Eicker. 1991b. "Cultural studies on the genus Termitomyces in South Africa. II. Macro-and micromorphology of comb sporodochia." Mycological Research 95 (4):444451.

Bottomley, Averil M.., and Claude Fuller. 1921. The fungus food of certain termites: South African Biological Society.

Bourguignon, T., N. Lo, S. L. Cameron, J. Sobotnik, Y. Hayashi, S. Shigenobu, D. Watanabe, Y. Roisin, T. Miura, and T. A. Evans. 2015. "The Evolutionary History of Termites as Inferred from 66 Mitochondrial Genomes." Molecular Biology and Evolution 32 (2):406-421. doi: 10.1093/molbev/msu308.

Bourguignon, Thomas, Nathan Lo, Jan Šobotník, Simon YW Ho, Naeem lqbal, Eric Coissac, Maria Lee, Martin M Jendryka, David Sillam-Dussès, and Barbora Kř́žková. 2017. "Mitochondrial phylogenomics resolves the global spread of higher termites, ecosystem engineers of the tropics." Molecular Biology and Evolution 34 (3):589-597.

Branstetter, Michael G, Ana Ješovnik, Jeffrey Sosa-Calvo, Michael W Lloyd, Brant C Faircloth, Seán G Brady, and Ted R Schultz. 2017. "Dry habitats were crucibles of domestication in the evolution of agriculture in ants." Proceedings of the Royal Society B: Biological Sciences 284 (1852):20170095.

Brefeld, O. 1889. Untersuchungen aus dem Gesammtgebiet der Mykologie. Fortsetzung der Schimmel, und Hefenpilze. Vol. 8, Basidiomyceten III. Leipzig.

Bretagnolle, F., and J. D. Thompson. 1995. "Gametes with the somatic chromosome number: mechanisms of their formation and role in the evolution of autopolyploid plants." New Phytologist 129 (1):1-22. doi: DOI 10.1111/i.1469-8137.1995.tb03005.x.

Brugerolle, G, and R Radek. 2006. "Symbiotic protozoa of termites." In Intestinal microorganisms of termites and other invertebrates, 243-269. Springer.

Bucek, A., J. Sobotnik, S. He, M. Shi, D. P. McMahon, E. C. Holmes, Y. Roisin, N. Lo, and T. Bourguignon. 2019. "Evolution of Termite Symbiosis Informed by Transcriptome-Based Phylogenies." Current Biology 29 (21):3728-3734 e4. doi: 10.1016/i.cub.2019.08.076.

Bull, J. J., and W. R. Rice. 1991. "Distinguishing mechanisms for the evolution of co-operation." J Theor Biol 149 (1):63-74. doi: 10.1016/s0022-5193(05)80072-4.

Buller, A. H. R. 1924. Agarics which are parasitic on other agarics, Researches on Fungi III. London: Longmans Green and Co.

Buller, A. H. R. 1934. Researches on fungi VI. London: Longmans, Green \& Company.

Burnetti, A., and W.C. Ratcliff. 2020. "The Origin of Phototrophy Reveals the Importance of Priority Effects for Evolutionary Innovation." Preprints (20201 10700).

Campbell, A. H. 1938. "Contribution to the biology of Collybia radicata (Relh.) Berk." Transactions of the British Mycological Society 22 (1-2):151-159. doi: 10.1080/00275514.1991.12025978. 
Casselton, Lorna A, and Natalie S Olesnicky. 1998. "Molecular genetics of mating recognition in basidiomycete fungi." Microbiology and molecular biology reviews 62 (1):55-70.

Castresana, J. 2000. "Selection of conserved blocks from multiple alignments for their use in phylogenetic analysis." Molecular Biology and Evolution 17 (4):540-552. doi: 10.1093/oxfordjournals.molbev.a026334.

Chiu, C. I., J. H. Ou, C. Y. Chen, and H. F. Li. 2019. "Fungal nutrition allocation enhances mutualism with fungus-growing termite." Fungal Ecology 41:92-100. doi: 10.1016/i.funeco.2019.04.001.

Chouvenc, T., C. A. Efstathion, M. L. Elliott, and N. Y. Su. 2013. "Extended disease resistance emerging from the faecal nest of a subterranean termite." Proc Biol Sci 280 (1770):20131885. doi: $10.1098 /$ rspb.2013.1885.

Chouvenc, T., M. L. Elliott, J. Sobotnik, C. A. Efstathion, and N. Y. Su. 2018. "The Termite Fecal Nest: A Framework for the Opportunistic Acquisition of Beneficial Soil Streptomyces (Actinomycetales: Streptomycetaceae)." Environ Entomol 47 (6):1431-1439. doi: $10.1093 /$ ee/nvy 152.

Chouvenc, T., J. Sobotnik, M. S. Engel, and T. Bourguignon. 2021. "Termite evolution: mutualistic associations, key innovations, and the rise of Termitidae." Cellular and Molecular Life Sciences 78 (6):2749-2769. doi: 10.1007/s00018-020-03728-z.

Chouvenc, Thomas, Paul Bardunias, Caroline A Efstathion, Seemanti Chakrabarti, Monica L Elliott, Robin Giblin-Davis, and Nan-Yao Su. 2013. "Resource opportunities from the nest of dying subterranean termite (Isoptera: Rhinotermitidae) colonies: a laboratory case of ecological succession." Annals of the Entomological Society of America 106 (6):771-778.

Clarke, L. J., J. Soubrier, L. S. Weyrich, and A. Cooper. 2014. "Environmental metabarcodes for insects: in silico PCR reveals potential for taxonomic bias." Molecular ecology resources 14 (6): $1160-70$. doi: 10.1111/1755-0998.12265.

Clémençon, H. 1997. Anatomie der Hymenomyceten. (Anatomy of the Hymenomycetes). Teufen, Switzerland: Flück-Wirth.

Cooper, J. A. 2014. "New species and combinations of some New Zealand agarics belonging to Clitopilus, Lyophyllum, Gerhardtia, Clitocybe, Hydnangium, Mycena, Rhodocollybia and Gerronema." Mycosphere 5 (2):263-288. doi: 10.5943/mycosphere/5/2/2.

Corner, Edred John Henry. 1994. "Agarics in Malesia. I. Tricholomatoid. II Mycenoid." Beihefte zur Nova Hedwigia 109:1-271.

Cragg, Simon M, Gregg T Beckham, Neil C Bruce, Timothy DH Bugg, Daniel L Distel, Paul Dupree, Amaia Green Etxabe, Barry S Goodell, Jody Jellison, and John E McGeehan. 2015. "Lignocellulose degradation mechanisms across the Tree of Life." Current Opinion in Chemical Biology 29:108-119.

da Costa, Rafael R, Sabine ME Vreeburg, Jonathan Z Shik, Duur K Aanen, and Michael Poulsen. 2019. "Can interaction specificity in the fungus-farming termite symbiosis be explained by nutritional requirements of the fungal crop?" Fungal Ecology 38:54-61.

Darlington, J. P. E. C. 1994. "Nutrition and evolution in fungus-growing termites." Nourishment and evolution in insect societies: 105-130.

de Bary, A. 1859. Zur kenntniss einiger Agaricinen. Vol. 17. Botanische Zeitung.

De Bie, T., N. Cristianini, J. P. Demuth, and M. W. Hahn. 2006. "CAFE: a computational tool for the study of gene family evolution." Bioinformatics 22 (10):1269-71. doi: $10.1093 /$ bioinformatics/b+l097.

De Duve, Christian. 2005. Singularities: landmarks on the pathways of life: Cambridge University Press.

De Fine Licht, H. H., A. Andersen, and D. K. Aanen. 2005. "Termitomyces sp. associated with the termite Macrotermes natalensis has a heterothallic mating system and multinucleate cells." Mycological Research 109 (Pt 3):314-8. doi: 10.1017/s0953756204001844.

de Fine Licht, H. H., J. J. Boomsma, and D. K. Aanen. 2006. "Presumptive horizontal symbiont transmission in the fungus-growing termite Macrotermes natalensis." Mol Ecol 15 (11):3131-8. doi: $10.1111 /$ i.1365-294X.2006.03008.x.

De Fine Licht, H. H., J. J. Boomsma, and A. Tunlid. 2014. "Symbiotic adaptations in the fungal cultivar of leaf-cutting ants." Nat Commun 5 (1):5675. doi: 10.1038/ncomms6675.

De Queiroz, K. 2007. "Species concepts and species delimitation." Systematic biology 56 (6):87986. doi: $10.1080 / 10635150701701083$. 
Diamond, J. 2002. "Evolution, consequences and future of plant and animal domestication." Nature 418 (6898):700-7. doi: 10.1038/nature01019.

Diamond, J. M., and D. Ordunio. 1999. Guns, germs, and steel: Books on Tape.

Donk, Marinus Anton. 1962. "The generic names proposed for Agaricaceae." Beihefte zur Nova Hedwigia 5:320.

Eberhardt, U., H. J. Beker, N. Schutz, M. Mikami, and T. Kasuya. 2020. "Rooting Hebelomas: The Japanese 'Hebeloma radicosum' is a distinct species, Hebeloma sagarae sp. nov. (Hymenogastraceae, Agaricales)." Phytotaxa 456 (2):125-144. doi: 10.1 1646/phytotaxa.456.2.1.

Eduardo, Nouhra, Soteras Florencia, Pastor Nicolás, and Geml József. 2018. "Richness, species composition and functional groups in Agaricomycetes communities along a vegetation and elevational gradient in the Andean Yungas of Argentina." Biodiversity and Conservation 27 (8):1849-1871.

Eggleton, P, T Abe, DE Bignell, and M Higashi. 2000. "Termites: evolution, sociality, symbioses, ecology." Netherlands: Springer.

Eggleton, P. 2000. "Global patterns of termite diversity." In Termites: evolution, sociality, symbioses, ecology, 25-51. Springer.

Eggleton, P. 2006. "The termite gut habitat: its evolution and co-evolution." In Intestinal microorganisms of termites and other invertebrates, edited by H. König and A. Varma, 373404. Springer.

Epp, L. S., S. Boessenkool, E. P. Bellemain, J. Haile, A. Esposito, T. Riaz, C. Erseus, V. I. Gusarov, M. E. Edwards, A. Johnsen, H. K. Stenoien, K. Hassel, H. Kauserud, N. G. Yoccoz, K. A. Brathen, E. Willerslev, P. Taberlet, E. Coissac, and C. Brochmann. 2012. "New environmental metabarcodes for analysing soil DNA: potential for studying past and present ecosystems." Molecular Ecology 21 (8):1821-33. doi: 10.1111/j.1365-294X.201 2.05537.x.

Evangelista, Dominic A, Benjamin Wipfler, Olivier Bethoux, Alexander Donath, Mari Fujita, Manpreet K Kohli, Frédéric Legendre, Shanlin Liu, Ryuichiro Machida, and Bernhard Misof. 2019. "An integrative phylogenomic approach illuminates the evolutionary history of cockroaches and termites (Blattodea)." Proceedings of the Royal Society B 286 (1895):20182076.

Farrell, Brian D, Andrea S Sequeira, Brian C O'Meara, Benjamin B Normark, Jeffrey H Chung, and Biarte H Jordal. 2001. "The evolution of agriculture in beetles (Curculionidae: Scolytinae and Platypodinae)." Evolution 55 (10):2011-2027.

Floudas, D., M. Binder, R. Riley, K. Barry, R. A. Blanchette, B. Henrissat, A. T. Martinez, R. Otillar, J. W. Spatafora, J. S. Yadav, A. Aerts, I. Benoit, A. Boyd, A. Carlson, A. Copeland, P. M. Coutinho, R. P. de Vries, P. Ferreira, K. Findley, B. Foster, J. Gaskell, D. Glotzer, P. Gorecki, J. Heitman, C. Hesse, C. Hori, K. Igarashi, J. A. Jurgens, N. Kallen, P. Kersten, A. Kohler, U. Kues, T. K. Kumar, A. Kuo, K. LaButti, L. F. Larrondo, E. Lindquist, A. Ling, V. Lombard, S. Lucas, T. Lundell, R. Martin, D. J. McLaughlin, I. Morgenstern, E. Morin, C. Murat, L. G. Nagy, M. Nolan, R. A. Ohm, A. Patyshakuliyeva, A. Rokas, F. J. Ruiz-Duenas, G. Sabat, A. Salamov, M. Samejima, J. Schmutz, J. C. Slot, F. St John, J. Stenlid, H. Sun, S. Sun, K. Syed, A. Tsang, A. Wiebenga, D. Young, A. Pisabarro, D. C. Eastwood, F. Martin, D. Cullen, I. V. Grigoriev, and D. S. Hibbett. 2012. "The Paleozoic origin of enzymatic lignin decomposition reconstructed from 31 fungal genomes." Science 336 (6089): 1715-9. doi: $10.1126 /$ science.1221748.

Folmer, O., M. Black, W. Hoeh, R. Lutz, and R. Vrijenhoek. 1994. "DNA primers for amplification of mitochondrial cytochrome c oxidase subunit I from diverse metazoan invertebrates." Molecular Marine Biology and Biotechnology 3 (5):294-9.

Foster, K. R., and T. Wenseleers. 2006. "A general model for the evolution of mutualisms." J Evol Biol 19 (4):1283-93. doi: 10.1111 /i.1420-9101.2005.01073.x.

Francke-Grosmann, H. 1956. "Hautdrüsen als träger der pilzsymbiose bei ambrosiakäfern." Zeitschrift für Morphologie und Ökologie der Tiere 45 (3):275-308. doi: $10.1007 /$ bf00430256.

Francke-Grosmann, H. 1967. "Ectosymbiosis in wood-inhabiting insects." In Symbiosis, edited by S.M. Henry, 141-205. New York: Academic Press.

Frank, S. A. 1994. "Genetics of mutualism: the evolution of altruism between species." J Theor Biol 170 (4):393-400. doi: 10.1006/jtbi.1994.1200. 
Frank, S. A. 1996a. "Host-symbiont conflict over the mixing of symbiotic lineages." Proc Biol Sci 263 (1368):339-44. doi: 10.1098/rspb.1996.0052.

Frank, S. A. 1996b. "Host control of symbiont transmission: The separation of symbionts into germ and soma." American Naturalist 148 (6):1 113-1124. doi: Doi 10.1086/285974.

Frank, S. A. 1997. "Models of symbiosis." Am Nat 150 Suppl 1 (S1):S80-99. doi: 10.1086/286051.

Froslev, T. G., D. K. Aanen, T. Laessoe, and S. Rosendahl. 2003. "Phylogenetic relationships of Termitomyces and related taxa." Mycol Res 107 (Pt 1 1):1277-86. doi: $10.1017 / \mathrm{s} 0953756203008670$.

Frøslev, T. G., D. K. Aanen, T. Laessoe, and S. Rosendahl. 2003. "Phylogenetic relationships of Termitomyces and related taxa." Mycol Res 107 (Pt 1 1):1277-86. doi: $10.1017 /$ s0953756203008670.

Fukiharu, Toshimitsu, and Tsuguo Hongo. 1995. "Ammonia fungi of Iriomote Island in the southern Ryukyus, Japan and a new ammonia fungus, Hebeloma luchuense." Mycoscience 36 (4):425-430.

Gardes, M., and T. D. Bruns. 1993. "ITS primers with enhanced specificity for basidiomycetes application to the identification of mycorrhizae and rusts." Molecular Ecology 2 (2):113. 8. doi: $10.1111 /$ j.1365-294x.1993.tb00005.x.

Garnier-Sillam, E., F. Toutain, G. Villemin, and J. Renoux. 1989. "Études Préliminaires des Meules Originales du Termite XylophageSphaerotermes sphaerothorax (Sjostedt)." Insectes sociaux $36(4): 293-312$.

Grassé, P, and Ch Noirot. 1948. "Sur le nid et la biologie du Sphaerotermes sphaerothorax (Sjöstedt)." Annales des Sciences Naturelles, Zoologie.

Green, AM, UG Mueller, and RMM Adams. 2002. "Extensive exchange of fungal cultivars between sympatric species of fungus-growing ants." Molecular Ecology 11 (2):191-195.

Griffith, G. W., K. P. Gajda, A. P. Detheridge, B. Douglas, J. Bingham, A. Turner, V. Bowmaker, D. A. Evans, W. G. McAdoo, and B. T. M. Dentinger. 2019. "Strangler unmasked: Parasitism of Cystoderma amianthinum by Squamanita paradoxa and S. pearsonii." Fungal Ecology 39:131-141. doi: 10.1016/i.funeco.2018.11.012.

Hammer, K. 1984. "Das domestikationssyndrom." Die Kulturpflanze 32 (1):1 1-34.

Harlan, J. R., J. M. J. de Wet, and E. G. Price. 1973. "Comparative Evolution of Cereals." Evolution 27 (2):311-325. doi: $10.1111 /$ i.1558-5646.1973.tb00676.x.

Harrington, T. C. 1987. "New Combinations in Ophiostoma of Ceratocystis Species with Leptographium Anamorphs." Mycotaxon 28 (1):39-43.

Harrington, Thomas C. 2005. "Ecology and evolution of mycophagous bark beetles and their fungal partners." In Insect-Fungal Associations: Ecology and Evolution, edited by M. Blackwell F.E. Vega, 257-291. Oxford: Oxford Univeristy Press.

Hausberger, B., D. Kimpel, A. van Neer, and J. Korb. 2011 . "Uncovering cryptic species diversity of a termite community in a West African savanna." Mol Phylogenet Evol 61 (3):964-9. doi: 10.1016/i.ympev.2011.08.015.

Hawksworth, David L, David S Hibbett, Paul M Kirk, and Robert Lücking. 2016. "Proposals to amend the Codes. 308-310 Proposals to permit DNA sequence data to serve as types of names of fungi." Tax 65 (4):899-900.

Hedges, Lauren M, Jeremy C Brownlie, Scott L O'Neill, and Karyn N Johnson. 2008. "Wolbachia and virus protection in insects." Science 322 (5902):702-702.

Heim, R. 1942. "Nouvelles études descriptives sur les agarics termitophiles d'Afrique tropicale." Archives du Muséum National d'Histoire Naturelle 18:107-166.

Heim, R. 1951. "Les Termitomyces du Congo belge recueillis par Madame M. Goossens-Fontana." Bulletin du Jardin botanique de I'Etat, Bruxelles/Bulletin van den Rijksplantentuin, Brussel:205-222.

Heim, R. 1977. Termites et champignons; les champignons termitophiles d'Afrique noire et d'Asie meridionale. Paris: Editions Boubée.

Herre, E. A., N. Knowlton, U. G. Mueller, and S. A. Rehner. 1999. "The evolution of mutualisms: exploring the paths between conflict and cooperation." Trends Ecol Evol 14 (2):49-53. doi: 10.1016/s0169-5347(98)01529-8.

Hibbett, David S, and John W Taylor. 2013. "Fungal systematics: is a new age of enlightenment at hand?" Nature Reviews Microbiology 11 (2):1 29-133. 
Hilgenboecker, Kirsten, Peter Hammerstein, Peter Schlattmann, Arndt Telschow, and John H Werren. 2008. "How many species are infected with Wolbachia?-a statistical analysis of current data." FEMS microbiology letters 281 (2):215-220.

Hinze, B., K. Crailsheim, and R. H. Leuthold. 2002. "Polyethism in food processing and social organisation in the nest of Macrotermes bellicosus (Isoptera, Termitidae)." Insectes Sociaux 49 (1):31-37. doi: 10.1007/s00040-002-8275-1.

Hofstetter, V., H. Clemencon, R. Vilgalys, and J. M. Moncalvo. 2002. "Phylogenetic analyses of the Lyophylleae (Agaricales, Basidiomycota) based on nuclear and mitochondrial rDNA sequences." Mycological Research 106:1043-1059. doi: 10.1017/S095375620200641x.

Hofstetter, V., S. A. Redhead, F. Kauff, J. M. Moncalvo, P. B. Matheny, and R. Vilgalys. 2014. "Taxonomic revision and examination of ecological transitions of the Lyophyllaceae (Basidiomycota, Agaricales) based on a multigene phylogeny." Cryptogamie Mycologie 35 (4):399-425. doi: 10.7872/crym.v35.iss4.2014.399.

Horak, E. 1968. "Synopsis Generum Agaricalium (Die Gattungstypen der Agaricales)." Beitrage zur Kryptogamenflora der Schweiz 13.

Howe, Jack, Morten Schiøtt, and Jacobus J Boomsma. 2018. "Horizontal partner exchange does not preclude stable mutualism in fungus-growing ants." Behavioral Ecology.

Hughes, K. W., R. H. Petersen, J. E. Johnson, J. M. Moncalvo, R. Vilgalys, S. A. Redhead, T. Thomas, and L. L. McGhee. 2001. "Infragenic phylogeny of Collybia s. str. based on sequences of ribosomal ITS and LSU regions." Mycological Research 105 (2):164-172. doi: Doi $10.1017 /$ S0953756200003415.

Hulcr, J., and A. I. Cognato. 2010. "Repeated evolution of crop theft in fungus-farming ambrosia beetles." Evolution 64 (11):3205-12. doi: 10.1111/i.1558-5646.2010.01055.x.

Hulcr, J., and L. L. Stelinski. 2017. "The Ambrosia Symbiosis: From Evolutionary Ecology to Practical Management." Annu Rev Entomol 62:285-303. doi: 10.1146/annurev-ento-031616035105.

Ingold, C. T. 1940. "Note on the distribution of basidia in fruit bodies of Nyctalis parasitica (Bull.) fr." Transactions of the British Mycological Society 24 (1):29-32.

Ingold, Cecil Terence. 1953. Dispersal in fungi. Vol. 76: LWW.

Inward, D., G. Beccaloni, and P. Eggleton. 2007. "Death of an order: a comprehensive molecular phylogenetic study confirms that termites are eusocial cockroaches." Biol Lett 3 (3):331-5. doi: $10.1098 / \mathrm{rsbl} .2007 .0102$.

Inward, D. J., A. P. Vogler, and P. Eggleton. 2007. "A comprehensive phylogenetic analysis of termites (Isoptera) illuminates key aspects of their evolutionary biology." Mol Phylogenet Evol 44 (3):953-67. doi: 10.1016/i.ympev.2007.05.014.

Ivany, Linda C, William P Patterson, and Kyger C Lohmann. 2000. "Cooler winters as a possible cause of mass extinctions at the Eocene/Oligocene boundary." Nature 407 (6806):887890.

Izhar, A., A. N. Khalid, and H. Bashir. 2020. "Termitomyces sheikhupurensis sp. nov. (Lyophyllaceae, Agaricales) from Pakistan, evidence from morphology and DNA sequences data." Turkish Journal of Botany 44 (6):694-704. doi: 10.3906/bot-2003-51.

Jarvis, A., and M. Mulligan. 2011 . "The climate of cloud forests." Hydrological Processes 25 (3):327343. doi: $10.1002 /$ hyp.7847.

Ješovnik, A., V. L. González, and T. R. Schultz. 2016. "Phylogenomics and Divergence Dating of Fungus-Farming Ants (Hymenoptera: Formicidae) of the Genera Sericomyrmex and Apterostigma." PLoS One 11 (7):e0151059. doi: 10.1371/journal.pone.0151059.

Johnson, R. A., R. J. Thomas, T. G. Wood, and M. J. Swift. 1981. "The Inoculation of the Fungus Comb in Newly Founded Colonies of Some Species of the Macrotermitinae (Isoptera) from Nigeria." Journal of Natural History 15 (5):751-756. doi: Doi $10.1080 / 00222938100770541$.

Johnson, RA. 1981. "Colony development and estabilishment of fungus comb in Microtermes. umbaricus Siöstedt (Isoptera, Macrotermitinae) from Nigeria." J Nat Hist 32:3-12.

Jouquet, P., S. Traore, C. Choosai, C. Hartmann, and D. Bignell. 2011. "Influence of termites on ecosystem functioning. Ecosystem services provided by termites." European Journal of Soil Biology 47 (4):215-222. doi: 10.1016/i.ejsobi.201 1.05.005. 
Jusino, M. A., M. T. Banik, J. M. Palmer, A. K. Wray, L. Xiao, E. Pelton, J. R. Barber, A. Y. Kawahara, C. Gratton, M. Z. Peery, and D. L. Lindner. 2019. "An improved method for utilizing highthroughput amplicon sequencing to determine the diets of insectivorous animals." Molecular ecology resources 19 (1):176-190. doi: 10.1111/1755-0998.12951.

Kalamees, K. 1992. "Tricholomella, a New Genus, with the Distribution Data of TricholomellaConstrictum, Comb-Nov in East Europe and Asia." Persoonia 14 (4):445-447.

Katoh, H., T. Miura, K. Maekawa, N. Shinzato, and T. Matsumoto. 2002. "Genetic variation of symbiotic fungi cultivated by the macrotermitine termite Odontotermes formosanus (Isoptera: Termitidae) in the Ryukyu Archipelago." Mol Ecol 11 (8):1565-72. doi: 10.1046/i.1365-294x.2002.01535.x.

Katoh, K., and D. M. Standley. 2013. "MAFFT multiple sequence alignment software version 7: improvements in performance and usability." Molecular biology and evolution 30 (4):77280. doi: $10.1093 / \mathrm{molbev} / \mathrm{mst0} 10$.

Katoh, Kazutaka, John Rozewicki, and Kazunori D Yamada. 2017. "MAFFT online service: multiple sequence alignment, interactive sequence choice and visualization." Briefings in bioinformatics.

Kauserud, H., and T. Schumacher. 2001. "Outcrossing or inbreeding: DNA markers provide evidence for type of reproductive mode in Phellinus nigrolimitatus (Basidiomycota)." Mycological Research 105 (6):676-683. doi: Doi 10.1017/S0953756201004191.

Kearse, M., R. Moir, A. Wilson, S. Stones-Havas, M. Cheung, S. Sturrock, S. Buxton, A. Cooper, S. Markowitz, C. Duran, T. Thierer, B. Ashton, P. Meintjes, and A. Drummond. 2012 . "Geneious Basic: an integrated and extendable desktop software platform for the organization and analysis of sequence data." Bioinformatics 28 (12):1647-9. doi: 10.1093/bioinformatics/bts 199.

Kent, D. S., and J. A. Simpson. 1992. "Eusociality in the beetle Austroplatypus incompertus (Coleoptera: Curculionidae)." Naturwissenschaften $79 \quad$ (2):86-87. doi: $10.1007 /$ bf01131810.

Kiers, E. T., R. A. Rousseau, S. A. West, and R. F. Denison. 2003. "Host sanctions and the legumerhizobium mutualism." Nature 425 (6953):78-81. doi: 10.1038/nature01931.

Kirk, Paul M, Paul F Cannon, DW Minter, and JA Stalpers. 2008. "Dictionary of the Fungi.(10thedn)." Wallingford, UK.

Klepzig, K. D., and D. L. Six. 2004. "Bark beetle-fungal symbiosis: Context dependency in complex associations." Symbiosis 37 (1-3): 189-205.

Knudsen, Henning, and Jan Vesterholt. 2008. Funga nordica: Nordsvamp.

Koné, N'golo Abdoulaye, Bakary Soro, Linda Patricia Louyounan Vanié-Léabo, Souleymane Konaté, Adama Bakayoko, and Daouda Koné. 2018. "Diversity, phenology and distribution of Termitomyces species in Côte d'lvoire." Mycology 9 (4):307-315.

Koné, NA, K Dosso, S Konaté, JY Kouadio, and KE Linsenmair. 2011 . "Environmental and biological determinants of Termitomyces species seasonal fructification in central and southern Côte d'Ivoire." Insectes sociaux 58 (3):371-382.

Kooij, P. W., D. K. Aanen, M. Schiøtt, and J. J. Boomsma. 2015. "Evolutionarily advanced ant farmers rear polyploid fungal crops." Journal of evolutionary biology 28 (11):1911-24. doi: 10.1111 /jeb.12718.

Korb, J., and D. K. Aanen. 2003. "The evolution of uniparental transmission of fungal symbionts in fungus-growing termites (Macrotermitinae)." Behavioral Ecology and Sociobiology 53 (2):65-71. doi: 10.1007/s00265-002-0559-y.

Korb, J., B. D. Kasseney, Y. T. Cakpo, R. H. Casalla Daza, Jnkb Gbenyedji, M. E. Ilboudo, G. Josens, N. A. Kone, K. Meusemann, A. B. Ndiaye, S. I. Okweche, M. Poulsen, Y. Roisin, and F. Sankara. 2019. "Termite Taxonomy, Challenges and Prospects: West Africa, A Case Example." Insects 10 (1):32. doi: 10.3390/insects 10010032.

Kornerup, Andreas, and Johan Henrik Wanscher. 1978. Methuen's handbook of colour. 3 ed.

Krombholz, J. V. 1831. Naturgetreue Abbildungen und Beschreibungen der essbaren, schädlichen und verdächtigen Schwämme. Prague: G. Calve.

Kubatko, L. S., and J. H. Degnan. 2007. "Inconsistency of phylogenetic estimates from concatenated data under coalescence." Systematic Biology 56 (1):17-24. doi: $10.1080 / 10635150601146041$. 
Kües, U., S. M. Badalyan, A. Gießler, and B. Dörnte. 2016. "12 Asexual Sporulation in Agaricomycetes." In The Mycota I, 3rd edition. Growth, Differentiation and Sexuality, edited by Wendland J., 269-328. Springer.

Kües, U., and M. Navarro-González. 2015. "How do Agaricomycetes shape their fruiting bodies? 1. Morphological aspects of development." Fungal Biology Reviews 29 (2):63-97. doi: 10.1016/i.fbr.2015.05.001.

Kuhn, G., M. Hijri, and I. R. Sanders. 2001. "Evidence for the evolution of multiple genomes in arbuscular mycorrhizal fungi." Nature 414 (6865):745-8. doi: 10.1038/414745a.

Lcessøe, T., O. S. Pedersen, and P. Sysouphanthong. 2019. "An introduction to the edible, poisonous and medicinal fungi of northern Laos." Laos: Agro-Biodiversity Project.

Law, R., and U. Dieckmann. 1998. "Symbiosis through exploitation and the merger of lineages in evolution." Proceedings of the Royal Society B-Biological Sciences 265 (1402):1 245-1253. doi: DOI 10.1098/rspb.1998.0426.

Lennox, J. W. 1979. "Collybioid Genera in the Pacific Northwest." Mycotaxon 9 (1):1 17-231.

Leuthold, R. H., S. Badertscher, and H. Imboden. 1989. "The inoculation of newly formed fungus comb with Termitomyces in Macrotermes colonies (Isoptera, Macrotermitinae)." Insectes Sociaux $36(4): 328-338$.

Li, Y., C. C. Bateman, J. Skelton, M. A. Jusino, Z. J. Nolen, D. R. Simmons, and J. Hulcr. 2017. "Wood decay fungus Flavodon ambrosius (Basidiomycota: Polyporales) is widely farmed by two genera of ambrosia beetles." Fungal Biology 121 (11):984-989. doi: 10.1016/i.funbio.2017.08.004.

Lo, Nathan, Michael S Engel, Stephen Cameron, Christine A Nalepa, Gaku Tokuda, David Grimaldi, Osamu Kitade, Kumar Krishna, Klaus-Dieter Klass, and Kiyoto Maekawa. 2007. "Save Isoptera: A comment on Inward et al." Biology letters 3 (5):562-563.

Lücking, R., M. C. Aime, B. Robbertse, A. N. Miller, H. A. Ariyawansa, T. Aoki, G. Cardinali, P. W. Crous, I. S. Druzhinina, and D. M. Geiser. 2020. "Unambiguous identification of fungi: where do we stand and how accurate and precise is fungal DNA barcoding?" IMA fungus $11(1): 1-32$.

Maeno, Jun, Kiminori Shimizu, Naohiko Sagara, and Chihiro Tanaka. 2016. "Identity between the fruit bodies of Hebeloma radicosum and the mycosymbiont in ectomycorrhizas colonizing mole latrines." mycoscience 57 (3): 196-199.

Makonde, Huxley M, Hamadi I Boga, Zipporah Osiemo, Romano Mwirichia, J Benjamin Stielow, Markus Göker, and Hans-Peter Klenk. 2013. "Diversity of Termitomyces associated with fungus-farming termites assessed by cultural and culture-independent methods." PloS one 8 (2):e56464.

Malaisse, F. 1978. "High termitaria." In Biogeography and Ecology of Southern Africa, edited by M. J. A. Werger, 1279-1300. Dordrecht: Springer Netherlands.

Malloch, David, and Meredith Blackwell. 1992. "Dispersal of fungal diaspores." The fungal community: its organization and role in the ecosystem: 147-171.

Martin, M. M., and J. S. Martin. 1978. "Cellulose Digestion in the Midgut of the Fungus-Growing Termite Macrotermes natalensis: The Role of Acquired Digestive Enzymes." Science 199 (4336): 1453-5. doi: 10.1 126/science.199.4336.1453.

Matheny, P. B., J. M. Curtis, V. Hofstetter, M. C. Aime, J. M. Moncalvo, Z. W. Ge, J. C. Slot, J. F. Ammirati, T. J. Baroni, N. L. Bougher, K. W. Hughes, D. J. Lodge, R. W. Kerrigan, M. T. Seidl, D. K. Aanen, M. DeNitis, G. M. Daniele, D. E. Desjardin, B. R. Kropp, L. L. Norvell, A. Parker, E. C. Vellinga, R. Vilgalys, and D. S. Hibbett. 2006. "Major clades of Agaricales: a multilocus phylogenetic overview." Mycologia 98 (6):982-95. doi: 10.3852/mycologia.98.6.982.

Mayden, R. L. 1997. A hierarchy of species concepts: the denovement in the saga of the species problem. Edited by M. F. Claridge, Dawah, H. A. \& Wilson, M. R., Species: The units of diversity: Chapman \& Hall.

Mayers, C. G., T. C. Harrington, H. Masuya, B. H. Jordal, D. L. McNew, H. H. Shih, F. Roets, and G. J. Kietzka. 2020. "Patterns of coevolution between ambrosia beetle mycangia and the Ceratocystidaceae, with five new fungal genera and seven new species." Persoonia 44:41-66. doi: 10.3767/persoonia.2020.44.02.

Maynard-Smith, J., and E. Szathmáry. 1997. The Major Transitions in Evolution: Oxford University Press. 
Mayr, E. 2000. The biological species concept, Species concepts and phylogenetic theory: a debate. New York: Columbia University Press.

Mehdiabadi, N. J., and T. R. Schultz. 2010. "Natural history and phylogeny of the fungus-farming ants (Hymenoptera: Formicidae: Myrmicinae: Attini)." Myrmecological News 13:37-55.

Meyer, Rachel S, and Michael D Purugganan. 2013. "Evolution of crop species: genetics of domestication and diversification." Nature reviews genetics 14 (12):840-852.

Milla, Rubén, Colin P Osborne, Martin M Turcotte, and Cyrille Violle. 2015. "Plant domestication through an ecological lens." Trends in ecology \& evolution 30 (8):463-469.

Miller, Mark A, Wayne Pfeiffer, and Terri Schwartz. 2010. "Creating the CIPRES Science Gateway for inference of large phylogenetic trees." 2010 gateway computing environments workshop (GCE).

Minh, B. Q., M. W. Hahn, and R. Lanfear. 2020. "New Methods to Calculate Concordance Factors for Phylogenomic Datasets." Molecular Biology and Evolution 37 (9):2727-2733. doi: $10.1093 / \mathrm{molbev} / \mathrm{msaa} 106$.

Minh, B. Q., H. A. Schmidt, O. Chernomor, D. Schrempf, M. D. Woodhams, A. Von Haeseler, and R. Lanfear. 2020. "IQ-TREE 2: New models and efficient methods for phylogenetic inference in the genomic era." Molecular Biology and Evolution 37 (5):1530-1534.

Mirarab, S., R. Reaz, M. S. Bayzid, T. Zimmermann, M. S. Swenson, and T. Warnow. 2014. "ASTRAL: genome-scale coalescent-based species tree estimation." Bioinformatics 30 (17):i541-8. doi: $10.1093 /$ bioinformatics/btu462.

Moncalvo, J. M., S. A. Rehner, and R. Vilgalys. 1993. "Systematics of Lyophyllum Section Difformia Based on Evidence from Culture Studies and Ribosomal DNA-Sequences." Mycologia 85 (5):788-794. doi: 10.2307/3760610.

Mondiet, N., M. P. Dubois, and M. A. Selosse. 2007. "The enigmatic Squamanita odorata (Agaricales, Basidiomycota) is parasitic on Hebeloma mesophaeum." Mycological Research 111 (5):599-602. doi: 10.1016/i.mycres.2007.03.009.

Moriya, Shigeharu, Tetsushi Inove, Moriya Ohkuma, Taprab Yaovapa, Tohru Johijma, Poonpilai Suwanarit, Utaiwan Sangwanit, Charunee Vongkaluang, Napavarn Noparatnaraporn, and Toshiaki Kudo. 2005. "Fungal community analysis of fungus gardens in termite nests." Microbes and Environments 20 (4):243-252.

Mossebo, D. C., E. P. F. Essouman, M. C. Machouart, and C. Gueidan. 2017. "Phylogenetic relationships, taxonomic revision and new taxa of Termitomyces (Lyophyllaceae, Basidiomycota) inferred from combined nLSU- and mtSSU-rDNA sequences." Phytotaxa 321 (1):71-102. doi: 10.11646/phytotaxa.321.1.3.

Mueller, U. G. 2002. "Ant versus fungus versus mutualism: ant-cultivar conflict and the deconstruction of the attine ant-fungus symbiosis." The American Naturalist 160 Suppl 4 (S4):S67-98. doi: $10.1086 / 342084$.

Mueller, U. G., N. M. Gerardo, D. K. Aanen, D. L. Six, and T. R. Schultz. 2005. "The evolution of agriculture in insects." Annual Review of Ecology Evolution and Systematics 36:563-595. doi: 10.1146 /annurev.ecolsys.36.102003.152626.

Mueller, U. G., A. Ortiz, and M. Bacci. 2010. "Planting of fungus onto hibernating workers of the fungus-growing ant Mycetosoritis clorindae (Attini, Formicidae)." Insectes Sociaux 57 (2):209-21 5. doi: 10.1007/s00040-010-0072-7.

Mueller, U. G., T. R. Schultz, C. R. Currie, R. M. Adams, and D. Malloch. 2001. "The origin of the attine ant-fungus mutualism." Q Rev Biol 76 (2):169-97. doi: 10.1086/393867.

Mueller, Ulrich G, Stephen A Rehner, and Ted R Schultz. 1998. "The evolution of agriculture in ants." Science 281 (5385):2034-2038.

Mujinya, B. B., M. Adam, F. Mees, J. Bogaert, I. Vranken, H. Erens, G. Baert, M. Ngongo, and E. Van Ranst. 2014. "Spatial patterns and morphology of termite (Macrotermes falciger) mounds in the Upper Katanga, DR Congo." Catena 114:97-106. doi: 10.1016/i.catena.2013.10.015.

Munkacsi, A. B., J. J. Pan, P. Villesen, U. G. Mueller, M. Blackwell, and D. J. McLaughlin. 2004. "Convergent coevolution in the domestication of coral mushrooms by fungus-growing ants." Proceedings of the Royal Society of London. Series B: Biological Sciences 271 (1550):1777. 82. doi: 10.1098/rspb.2004.2759.

Natarajan, K. 1975. "South Indian Agaricales. I. Termitomyces." Kavaka 3:63-66.

Natarajan, K. 1977. "A new species of Termitomyces from India." Current Science 46:679-680. 
Nieuwenhuis, M., L. J. J. van de Peppel, F. T. Bakker, B. J. Zwaan, and D. K. Aanen. 2019. "Enrichment of G4DNA and a Large Inverted Repeat Coincide in the Mitochondrial Genomes of Termitomyces." Genome Biology and Evolution 11 (7):1857-1869. doi: 10.1093/gbe/evz122.

Nilsson, R. H., E. Kristiansson, M. Ryberg, N. Hallenberg, and K. H. Larsson. 2008. "Intraspecific ITS Variability in the Kingdom Fungi as Expressed in the International Sequence Databases and Its Implications for Molecular Species Identification." Evolutionary Bioinformatics 4:193-201.

Nilsson, R. H., M. Ryberg, E. Kristiansson, K. Abarenkov, K. H. Larsson, and U. Koljalg. 2006. "Taxonomic reliability of DNA sequences in public sequence databases: a fungal perspective." PLoS One 1 (1):e59. doi: 10.1371/journal.pone.0000059.

Nixon, K. C., and Q. D. Wheeler. 1990. "An Amplification of the Phylogenetic Species Concept." Cladistics 6 (3):211-223. doi: 10.1111/j.1096-0031.1990.tb00541.x.

Nobre, T., and D. K. Aanen. 2012 . "Fungiculture or Termite Husbandry? The Ruminant Hypothesis." Insects 3 (1):307-23. doi: 10.3390/insects3010307.

Nobre, T., P. Eggleton, and D. K. Aanen. 2010. "Vertical transmission as the key to the colonization of Madagascar by fungus-growing termites?" Proc Biol Sci 277 (1680):359-65. doi: 10.1098/rspb.2009.1373.

Nobre, T., C. Fernandes, J. J. Boomsma, J. Korb, and D. K. Aanen. 2011 . "Farming termites determine the genetic population structure of Termitomyces fungal symbionts." Mol Ecol 20 (9):202333. doi: $10.1111 /$ i.1365-294X.2011.05064.x.

Nobre, T., N. A. Kone, S. Konate, K. E. Linsenmair, and D. K. Aanen. 2011 . "Dating the fungus-growing termites' mutualism shows a mixture between ancient codiversification and recent symbiont dispersal across divergent hosts." Mol Ecol 20 (12):2619-27. doi: 10.1111/i.1365294X.201 1.05090.x.

Nobre, T., B. Koopmanschap, J. J. P. Baars, A. S. M. Sonnenberg, and D. K. Aanen. 2014. "The scope for nuclear selection within Termitomyces fungi associated with fungus-growing termites is limited." Bmc Evolutionary Biology 14 (1):1-12. doi: 10.1 186/1471-2148-14-121.

Nurk, S., A. Bankevich, D. Antipov, A. Gurevich, A. Korobeynikov, A. Lapidus, A. Priibelsky, A. Pyshkin, A. Sirotkin, Y. Sirotkin, R. Stepanauskas, J. McLean, R. Lasken, S. R. Clingenpeel, T. Woyke, G. Tesler, M. A. Alekseyev, and P. A. Pevzner. 2013. "Assembling Genomes and Minimetagenomes from Highly Chimeric Reads." Berlin, Heidelberg.

Okonechnikov, K., O. Golosova, M. Fursov, and Ugene team. 2012. "Unipro UGENE: a unified $\begin{array}{llll}\text { bioinformatics toolkit." } \quad \text { Bioinformatics } 28 & \text { (8): 1166-7. doi: }\end{array}$ 10.1093/bioinformatics/bts091.

Osiemo, Z. B., A. Marten, M. Kaib, L. M. Gitonga, H. I. Boga, and R. Brandl. 2010 . "Open relationships in the castles of clay: high diversity and low host specificity of Termitomyces fungi associated with fungus-growing termites in Africa." Insectes Sociaux 57 (3):351-363. doi: 10.1007/s00040-010-0092-3.

Otieno, NC. 1964. "Contributions to a knowledge of termite fungi in East Africa." Proceedings of the East African Academy 11:108-120.

Otieno, NC. 1968. "Further contributions to a knowledge of termite fungi in East Africa: the genus Termitomyces Heim." Sydowia Ann Mycol.

Palmer, J. \& Stajich, J. 2019. "nextgenusfs/funannotate: funannotate v1.5.3 (Version 1.5.3). Zenodo." doi: 10.5281 /zenodo.2604804.

Pannebakker, B. A., B. Loppin, C. P. Elemans, L. Humblot, and F. Vavre. 2007. "Parasitic inhibition of cell death facilitates symbiosis." Proc Natl Acad Sci U S A 104 (1):213-5. doi: 10.1073/pnas.0607845104.

Paradis, E., and K. Schliep. 2019. "ape 5.0: an environment for modern phylogenetics and evolutionary analyses in R." Bioinformatics $35 \quad(3): 526-528$. doi: 10.1093/bioinformatics/bty633.

Parker, Chas S. 1933. "A taxonomic study of the genus Hypholoma in North America." Mycologia $25(3): 160-212$.

Pearce, Michael J, Robert H Cowie, Angela S Pack, and Duncan Reavey. 1990. "Intraspecific aggression, colony identity and foraging distances in Sudanese Microtermes spp.(Isoptera: Termitidae: Macrotermitinae)." Ecological Entomology 15 (1):71-77. 
Pegler, David Norman. 1977. A preliminary agaric flora of East Africa: Her Majesty's Stationery Office.

Pegler, David Norman, and GD Piearce. 1980. "The edible mushrooms of Zambia." Kew bulletin:475-491.

Pegler, DN, and M Vanhaecke. 1994. "Termitomyces of southeast Asia." Kew Bulletin:717-736.

Pera, J., and I. F. Alvarez. 1995. "Ectomycorrhizal Fungi of Pinus-Pinaster." Mycorrhiza 5 (3):193200. doi: DOI $10.1007 /$ s005720050059.

Piearce, GD. 1987. "The genus Termitomyces in Zambia." Mycologist 1 (3):111-116.

Poulsen, M., H. F. Hu, C. Li, Z. S. Chen, L. H. Xu, S. Otani, S. Nygaard, T. Nobre, S. Klaubauf, P. M. Schindler, F. Hauser, H. L. Pan, Z. K. Yang, A. S. M. Sonnenberg, Z. W. de Beer, Y. Zhang, M. J. Wingfield, C. J. P. Grimmelikhuijzen, R. P. de Vries, J. Korb, D. K. Aanen, J. Wang, J. J. Boomsma, and G. J. Zhang. 2014. "Complementary symbiont contributions to plant decomposition in a fungus-farming termite." Proceedings of the National Academy of Sciences of the United States of America 111 (40):14500-14505. doi: 10.1073/pnas.1319718111.

Puillandre, N., A. Lambert, S. Brouillet, and G. Achaz. 2012. "ABGD, Automatic Barcode Gap Discovery for primary species delimitation." Molecular Ecology 21 (8):1864-1877. doi: 10.1111/i.1365-294X.2011.05239.x.

Purugganan, M. D., and D. Q. Fuller. 2009. "The nature of selection during plant domestication." Nature 457 (7231):843-8. doi: 10.1038/nature07895.

Qin, J., E. Horak, F. Popa, K. H. Rexer, G. Kost, F. Li, and Z. L. Yang. 2018. "Species diversity, distribution patterns, and substrate specificity of Strobilurus." Mycologia 110 (3):584604. doi: 10.1080/00275514.2018.1463064.

Raja, H. A., A. N. Miller, C. J. Pearce, and N. H. Oberlies. 2017. "Fungal Identification Using Molecular Tools: A Primer for the Natural Products Research Community." Journal of Natural Products 80 (3):756-770. doi: 10.1021/acs.jnatprod.6b01085.

Redhead, S. A. 1981. "Parasitism of Bryophytes by Agarics." Canadian Journal of Botany 59 (1):6367. doi: DOI 10.1139/b81-011.

Redhead, S. A., J. F. Ammirati, G. R. Walker, L. L. Norvell, and M. B. Puccio. 1994. "Squamanita contortipes, the Rosetta Stone of a mycoparasitic agaric genus." Canadian Journal of Botany 72 (12): $1812-1824$. doi: 10.1139/b94-223.

Redhead, S. A., and D. W. Malloch. 1986. "The genus Phaeocollybia (Agaricales) in eastern Canada and its biological status." Canadian Journal of Botany 64 (6):1249-1254. doi: $10.1139 /$ b86-172.

Redhead, S. A., and K. A. Seifert. 2001. "Asterophora Ditmar ex Link 1809 versus Nyctalis Fries 1825, and the status of Ugola Adanson 1763." Taxon 50 (1):243-268.

Revell, L. J. 2009. "Size-correction and principal components for interspecific comparative studies." Evolution 63 (12):3258-68. doi: 10.1111/i.1558-5646.2009.00804.x.

Roberts, E. M., C. N. Todd, D. K. Aanen, T. Nobre, H. L. Hilbert-Wolf, P. M. O'Connor, L. Tapanila, C. Mtelela, and N. J. Stevens. 2016. "Oligocene Termite Nests with In Situ Fungus Gardens from the Rukwa Rift Basin, Tanzania, Support a Paleogene African Origin for Insect Agriculture." PLoS One 11 (6):e0156847. doi: 10.1371/journal.pone.0156847.

Ronquist, F., M. Teslenko, P. van der Mark, D. L. Ayres, A. Darling, S. Hohna, B. Larget, L. Liu, M. A. Suchard, and J. P. Huelsenbeck. 2012. "MrBayes 3.2: efficient Bayesian phylogenetic inference and model choice across a large model space." Syst Biol 61 (3):539-42. doi: 10.1093 /sysbio/sys029.

Rouland-Lefèvre, C. 2000. "Symbiosis with fungi." In Termites: evolution, sociality, symbioses, ecology, 289-306. Springer.

Rouland-Lefevre, Corinne, Michel Ngor Diouf, Alain Brauman, and Marc Neyra. 2002. "Phylogenetic relationships in Termitomyces (Family Agaricaceae) based on the nucleotide sequence of ITS: a first approach to elucidate the evolutionary history of the symbiosis between fungusgrowing termites and their fungi." Molecular phylogenetics and evolution 22 (3):423-429.

Rozas, J., A. Ferrer-Mata, J. C. Sanchez-DelBarrio, S. Guirao-Rico, P. Librado, S. E. Ramos-Onsins, and A. Sanchez-Gracia. 2017. "DnaSP 6: DNA Sequence Polymorphism Analysis of Large Data Sets." Molecular biology and evolution 34 (12):3299-3302. doi: $10.1093 / \mathrm{molbev} / \mathrm{msx} 248$. 
Saar, I., K. Põldmaa, and U. Kõljalg. 2009. "The phylogeny and taxonomy of genera Cystoderma and Cystodermella (Agaricales) based on nuclear ITS and LSU sequences." Mycological Progress 8 (1):59-73. doi: 10.1007/s1 1557-008-0578-9.

Sachs, J. L., U. G. Mueller, T. P. Wilcox, and J. J. Bull. 2004. "The evolution of cooperation." Q Rev Biol 79 (2):135-60. doi: 10.1086/383541.

Sachs, J. L., and E. L. Simms. 2006. "Pathways to mutualism breakdown." Trends in ecology \& evolution 21 (10):585-92. doi: 10.1016/j.tree.2006.06.018.

Sagan, L. 1967. "On the origin of mitosing cells." Journal of Theoretical Biology 14 (3):255-74. doi: 10.1016/0022-5193(67)90079-3.

Sagara, N, T Hongo, Y Murakami, T Hashimoto, H Nagamasu, T Fukiharu, and Y Asakawa. 2000a. "Hebeloma radicosoides sp. nov., an agaric belonging to the chemoecological group ammonia fungi." Mycological Research 104 (8):1017-1024.

Sagara, N, Y Kitamoto, R Nishio, and S Yoshimi. 1985. "Association of two Hebeloma species with decomposed nests of vespine wasps." Transactions of the British Mycological Society 84 (2):349-352.

Sagara, N., S. Honda, E. Kuroyanagi, and S. Takayama. 1981. "The Occurrence of HebelomaSpoliatum and Hebeloma-Radicosum on the Dung-Deposited Burrows of UrotrichusTalpoides (Shrew Mole)." Transactions of the Mycological Society of Japan 22 (4):441455.

Sagara, N., T. Hongo, Y. Murakami, T. Hashimoto, H. Nagamasu, T. Fukiharu, and Y. Asakawa. 2000b. "Hebeloma radicosoides sp nov., an agaric belonging to the chemoecological group ammonia fungi." Mycological Research 104 (8):1017-1024. doi: $10.1017 /$ S0953756299002439.

Sagara, N., B. Senn-Irlet, and P. Marstad. 2006. "Establishment of the case of Hebeloma radicosum growth on the latrine of the wood mouse." Mycoscience 47 (5):263-268.

Sagara, Naohiko. 1975. "Ammonia fungi: a chemoecological grouping of terrestrial fungi."

Sagara, Naohiko. 1995. "Association of ectomycorrhizal fungi with decomposed animal wastes in forest habitats: a cleaning symbiosis?" Canadian Journal of Botany 73 (S1):1423-1433.

Sagara, Naohiko, and Yu Fukasawa. 2014. "Inhabitant changes in long-term mole nesting at the same site, revealed by observing mushroom fruiting at the site." Mammalia 78 (3):383391.

Sagara, Naohiko, Jyuichi Ooyama, and Mamoru Koyama. 2008. "New causal animal for the growth of Hebeloma radicosum (Agaricales): shrew, Sorex sp.(Mammalia, Insectivora)." Mycoscience 49 (3):207-210.

Sands, William A. 1969. "The association of termites and fungi." Biology of termites 1:495-524.

Sattler, M. C., C. R. Carvalho, and W. R. Clarindo. 2016. "The polyploidy and its key role in plant breeding." Planta 243 (2):281-96. doi: 10.1007/s00425-015-2450-x.

Schlick-Steiner, B. C., F. M. Steiner, H. Konrad, B. Seifert, E. Christian, K. Moder, C. Stauffer, and R. H. Crozier. 2008. "Specificity and transmission mosaic of ant nest-wall fungi." Proc Natl Acad Sci U S A 105 (3):940-3. doi: 10.1073/pnas.0708320105.

Schoch, Conrad L, Keith A Seifert, Sabine Huhndorf, Vincent Robert, John L Spouge, C André Levesque, Wen Chen, and Fungal Barcoding Consortium. 2012. "Nuclear ribosomal internal transcribed spacer (ITS) region as a universal DNA barcode marker for Fungi." Proceedings of the National Academy of Sciences 109 (16):6241-6246.

Schremmer, Fritz. 1963. "Wechselbeziehungen zwischen Pilzen und Insekten. Beobachtungen an der Stinkmorchel, Phallus impudicus L. ex Pers." Österreichische botanische Zeitschrift 110 (4):380-400.

Schultz, T. R., J. Sosa-Calvo, S. G. Brady, C. T. Lopes, U. G. Mueller, M. Bacci, and H. L. Vasconcelos. 2015. "The Most Relictual Fungus-Farming Ant Species Cultivates the Most Recently Evolved and Highly Domesticated Fungal Symbiont Species." American Naturalist 185 (5):693-703. doi: 10.1086/680501.

Seppey, M., M. Manni, and E. M. Zdobnov. 2019. "BUSCO: assessing genome assembly and annotation completeness." In Gene Prediction, 227-245. Springer.

Shinzato, N., M. Muramatsu, Y. Watanabe, and T. Matsui. 2005. "Termite-regulated fungal monoculture in fungus combs of a macrotermitine termite Odontotermes formosanus." Zoolog Sci 22 (8):917-22. doi: 10.2108/zsj.22.917. 
Sigoillot, J., J. Berrin, M. Bey, L. Lesage-Meessen, A. Levasseur, A. Lomascolo, E. Record, and E. UzanBoukhris. 2012. "Fungal strategies for lignin degradation." In Advances in botanical research, 263-308. Elsevier.

Simon, C., F. Frati, A. Beckenbach, B. Crespi, H. Liu, and P. Flook. 1994. "Evolution, Weighting, and Phylogenetic Utility of Mitochondrial Gene-Sequences and a Compilation of Conserved Polymerase Chain-Reaction Primers." Annals of the Entomological Society of America 87 (6):651-701. doi: 10.1093/aesa/87.6.651.

Simpson, G. G. 1951. "The Species Concept." Evolution 5 (4):285-298. doi: Doi 10.2307/2405675. Singer, R. 1947. "New genera of fungi. III." Mycologia 39 (1):77-89.

Singer, R. 1962. "New genera of Fungi-VIII." Persoonia-Molecular Phylogeny and Evolution of Fungi $2(3): 407-415$.

Singer, R. 1970. Monograph No. 3. Omphalinae (Clitocybeae-Tricholomataceae, Basidiomycetes), Flora neotropica: Hafner Publishing, New York.

Slippers, B., P. De Groot, and M. J. Wingfield. 2011. The Sirex Woodwasp and its Fungal Symbiont: Research and Management of a Worldwide Invasive Pest. Dordrecht: Springer Science \& Business Media.

Slippers, B., B. P. Hurley, and M. J. Wingfield. 2015. "Sirex woodwasp: a model for evolving management paradigms of invasive forest pests." Annual review of entomology 60:60119. doi: 10.1146/annurev-ento-010814-021118.

Smith, Alexander Hanchett. 1947. "North American species of Mycena." North American species of Mycena.

Smith, M. E., T. W. Henkel, and J. A. Rollins. 2015. "How many fungi make sclerotia?" Fungal Ecology 13:211-220.

Šobotník, Jan, and Cecilia A. L. Dahlsïo. 2017. "Isoptera." In Reference Module in Life Sciences. Elsevier.

Staats, M., A. Cuenca, J. E. Richardson, R. Vrielink-van Ginkel, G. Petersen, O. Seberg, and F. T. Bakker. 2011 . "DNA damage in plant herbarium tissue." PLoS One 6 (12):e28448. doi: 10.1371 /journal.pone.0028448.

Stamatakis, A. 2014. "RAxML version 8: a tool for phylogenetic analysis and post-analysis of large phylogenies." Bioinformatics 30 (9):1312-3. doi: 10.1093/bioinformatics/btu033.

Stanke, M., and B. Morgenstern. 2005. "AUGUSTUS: a web server for gene prediction in eukaryotes that allows user-defined constraints." Nucleic Acids Research 33 (suppl_2):W465-W467. doi: $10.1093 /$ nar/gki458.

Stouthamer, R., J. A. Breeuwer, and G. D. Hurst. 1999. "Wolbachia pipientis: microbial manipulator of arthropod reproduction." Annu Rev Microbiol 53 (1):71-102. doi: 10.1146 /annurev.micro.53.1.71.

Szathmáry, E., and J. Maynard-Smith. 1995. "The major evolutionary transitions." Nature 374 (6519):227-32. doi: 10.1038/374227a0.

Szathmáry, Eörs. 2015. "Toward major evolutionary transitions theory 2.0." Proceedings of the National Academy of Sciences 112 (33):10104-10111.

Talbot, P. H. B. 1971. Principles of fungal taxonomy: Macmillan International Higher Education.

Talbot, PHB. 1977. "The Sirex-amylostereum-pinus association." Annual review of Phytopathology $15(1): 41-54$.

Thomas, R. J. 1981. "Ecological studies on the symbiosis of Termitomyces Heim with Nigerian Macrotermitinae."

Thompson, G. E. 1936. "Nyctalis parasitica and N. asterophora in culture." Mycologia 28 (3):222227.

Tibuhwa, DD, AK Kivaisi, and FSS Magingo. 2010. "Utility of the macro-micromorphological characteristics used in classifying the species of Termitomyces." Tanzania Journal of Science 36 (1).

Tibuhwa, Donatha Damian. 2012 . "Termitomyces species from Tanzania, their cultural properties and unequalled basidiospores." Journal of Biology and Life Science 3 (1):1 40-159.

Trifinopoulos, J., L. T. Nguyen, A. von Haeseler, and B. Q. Minh. 2016. "W-IQ-TREE: a fast online phylogenetic tool for maximum likelihood analysis." Nucleic Acids Res 44 (W1):W232-5. doi: $10.1093 / \mathrm{nar} / \mathrm{gkw} 256$.

Turland, Nick J, John Harry Wiersema, Fred R Barrie, Werner Greuter, David L Hawksworth, Patrick Stephen Herendeen, Sandra Knapp, Wolf-Henning Kusber, De-Zhu Li, and Karol Marhold. 
2018. International Code of Nomenclature for algae, fungi, and plants (Shenzhen Code) adopted by the Nineteenth International Botanical Congress Shenzhen, China, July 2017: Koeltz Botanical Books.

Usman, M., and A. N. Khalid. 2020. "Termitomyces acriumbonatus sp. nov. (Lyophyllaceae, Agaricales) from Pakistan." Phytotaxa 477 (2):217-228.

Uys, VM. 2002. A guide to the termite genera of southern Africa: Plant Protection Research Institute, Agricultural Research Council.

van de Peppel, L. J. J., D. K. Aanen, and P. H. W. Biedermann. 2018. "Low intraspecific genetic diversity indicates asexuality and vertical transmission in the fungal cultivars of ambrosia beetles." Fungal ecology 32:57-64.

van de Peppel, L.J.J., M. Nieuwenhuis, B. Auxier, A. Grum-Grzhimaylo, M. Cárdenas, Z. W. de Beer, D. J. Lodge, M. E. Smith, T. W. Kuyper, and A. E. Franco-Molano. 2021. "Ancestral Predisposition Towards a Domesticated Lifestyle in the Termite-Cultivated Fungus Termitomyces." doi: http://dx.doi.org/10.2139/ssrn.3828201.

van de Peppel, Lennart JJ, and Duur K Aanen. 2020. "High diversity and low host-specificity of Termitomyces symbionts cultivated by Microtermes spp. indicate frequent symbiont exchange." Fungal Ecology 45:100917.

Van der Westhuizen, GCA, and A Eicker. 1990. "Species of Termitomyces occurring in South Africa." Mycological Research 94 (7):923-937.

Van Waveren, E Kits. 1985. "The Dutch, French and British species of Psathyrella." PersooniaSupplement 2 (1):3-300.

Vanderpool, D., R. R. Bracewell, and J. P. McCutcheon. 2018. "Know your farmer: Ancient origins and multiple independent domestications of ambrosia beetle fungal cultivars." Mol Ecol 27 (8):2077-2094. doi: $10.1111 / \mathrm{mec} .14394$.

Vellinga, E. C., T. W. Kuyper, J. Ammirati, D. E. Desjardin, R. E. Halling, A. Justo, T. Laessoe, T. Lebel, D. J. Lodge, P. B. Matheny, A. S. Methven, P. A. Moreau, G. M. Mueller, M. E. Noordeloos, J. Nuytinck, C. L. Ovrebo, and A. Verbeken. 2015. "Six simple guidelines for introducing new genera of fungi." Ima Fungus 6 (2):65-68. doi: Doi 10.1007/Bf03449356.

Vesala, Risto, Tuula Niskanen, Kare Liimatainen, Hamadi Boga, Petri Pellikka, and Jouko Rikkinen. 2017. "Diversity of fungus-growing termites (Macrotermes) and their fungal symbionts (Termitomyces) in the semiarid Tsavo Ecosystem, Kenya." Biotropica 49 (3):402-412.

Viana, A. B., M. O. Côrtes, T. G. Cornelissen, and F. D. Neves. 2018 . "Interactions between woodinhabiting fungi and termites: a meta-analytical review." Arthropod-Plant Interactions 12 (2):229-235. doi: 10.1007/s1 1829-017-9570-0.

Vilgalys, R., and M. Hester. 1990. "Rapid Genetic Identification and Mapping of Enzymatically Amplified Ribosomal DNA from Several Cryptococcus Species." Journal of Bacteriology 172 (8):4238-4246. doi: DOI 10.1128/ib.172.8.4238-4246.1990.

Vizzini, A., G. Consiglio, L. Setti, and E. Ercole. 2015. "Calocybella, a new genus for Rugosomyces pudicus (Agaricales, Lyophyllaceae) and emendation of the genus Gerhardtia." IMA Fungus 6 (1): 1 - 11. doi: 10.5598/imafungus.2015.06.01.01.

Vo, T. L., U. G. Mueller, and A. S. Mikheyev. 2009. "Free-living fungal symbionts (Lepiotaceae) of fungus-growing ants (Attini: Formicidae)." Mycologia 101 (2):206-10. doi: 10.3852/07055.

Vogler, A. P., and R. DeSalle. 1994. "Evolution and phylogenetic information content of the ITS-1 region in the tiger beetle Cicindela dorsalis." Molecular Biology and Evolution 11 (3):393405. doi: 10.1093/oxfordjournals.molbev.a040121.

Vreeburg, S. M. E., N. C. A. de Ruijter, B. J. Zwaan, R. R. da Costa, M. Poulsen, and D. K. Aanen. 2020. "Asexual and sexual reproduction are two separate developmental pathways in a Termitomyces species." Biology Letters 16 (8):20200394. doi: 10.1098/rsbl.2020.0394.

Waller, D. A., J. P. Lafage, R. L. Gilbertson, and M. Blackwell. 1987. "Wood-Decay Fungi Associated with Subterranean Termites (Rhinotermitidae) in Louisiana." Proceedings of the Entomological Society of Washington 89 (3):417-424.

Walther, G., S. Garnica, and M. Weiss. 2005. "The systematic relevance of conidiogenesis modes in the gilled Agaricales." Mycological research 109 (Pt 5):525-44. doi: $10.1017 / \mathrm{s} 0953756205002868$.

Wang, B, and Y-L Qiu. 2006. "Phylogenetic distribution and evolution of mycorrhizas in land plants." Mycorrhiza 16 (5):299-363. 
Weber, Neal A. 1972. "The fungus-culturing behavior of ants." American Zoologist 12 (3):577-587.

Wei, T. Z., B. H. Tang, and Y. J. Yao. 2009. "Revision of Termitomyces in China." Mycotaxon 108 (1):257-285. doi: Doi 10.5248/108.257.

White, T. J., T. D. Bruns, S. J. W. T. Lee, and J. L. Taylor. 1990. "Amplification and direct sequencing of fungal ribosomal RNA genes for phylogenetics." PCR protocols: a guide to methods and applications 18 (1):315-322.

Wood, T. G. 1988. "Termites and the Soil Environment." Biology and Fertility of Soils 6 (3):228-236.

Wood, TG, and RJ Thomas. 1989. "The mutualistic association between Macrotermitinae and Termitomyces." Insect-fungus interactions:69-92.

Ye, L., S. C. Karunarathna, H. Li, J. Xu, K. D. Hyde, and P. E. Mortimer. 2019. "A Survey of Termitomyces (Lyophyllaceae, Agaricales), Including a New Species, from a Subtropical Forest in Xishuangbanna, China." Mycobiology 47 (4):391-400. doi: 10.1080/12298093.2019.1682449.

Zamora, J. C., M. Svensson, R. Kirschner, I. Olariaga, S. Ryman et al. 2018. "Considerations and consequences of allowing DNA sequence data as types of fungal taxa." Ima Fungus 9 (1):167-175. doi: 10.5598/imafungus.2018.09.01.10.

Zeale, M. R., R. K. Butlin, G. L. Barker, D. C. Lees, and G. Jones. 2011 . "Taxon-specific PCR for DNA barcoding arthropod prey in bat faeces." Molecular ecology resources 11 (2):236-44. doi: $10.1111 / \mathrm{j} .1755-0998.2010 .02920 . x$.

Zeder, M. A. 2012 2. "Pathways to animal domestication." Biodiversity in agriculture: domestication, evolution, and sustainability:227-259.

Zeder, M. A. 2015. "Core questions in domestication research." Proceedings of the National Academy of Sciences 112 (11):3191-8. doi: 10.1073/pnas.1501711112.

Zervakis, G. I., J. M. Moncalvo, and R. Vilgalys. 2004. "Molecular phylogeny, biogeography and speciation of the mushroom species Pleurotus cystidiosus and allied taxa." Microbiology 150 (Pt 3):715-726. doi: 10.1099/mic.0.26673-0.

Zhang, H., T. Yohe, L. Huang, S. Entwistle, P. Wu, Z. Yang, P. K. Busk, Y. Xu, and Y. Yin. 2018. "dbCAN2: a meta server for automated carbohydrate-active enzyme annotation." Nucleic acids research 46 (W1):W95-W101. doi: 10.1093/nar/gky418. 



\section{Summary}

Almost all living organisms on earth interact with other living organisms all the time. Long-term interactions between different organisms are called symbioses. A symbiosis can be beneficial for one party and neutral for the other party (commensalism), beneficial for one party and detrimental for the other party (parasitism) or beneficial for both parties (mutualism). Mutualistic symbioses have played an important role in development of life on earth and can be directly linked to major evolutionary innovations. In this thesis I focussed on mutualistic symbioses and on one obligate mutualistic symbiosis in particular; the fungus-growing termites and their fungal symbiont Termitomyces.

The origin of the symbiosis between fungus-growing termites (Macrotermitinae) and Basidiomycete fungi of the genus Termitomyces has been estimated at approximately 30 million years ago in the rainforests of central Africa. The symbiosis has a single origin with no known reversals of both partners to a non-symbiotic state. The single origin of fungiculture and the absence of any reversals of the fungus to a free-living state are puzzling as both parties have retained independent reproduction and dispersal, which could potentially destabilize the symbiosis by leaving room for the fungus to escape or for the termite to domesticate more than one symbiont. The single origin of fungiculture and the single successful domestication event are unique among the three main systems of fungus-growing insects (ants, beetles and termites), which raises questions on the origin of the symbiosis. For this thesis I studied the biology of the closest relatives of Termitomyces in order to reconstruct the biology of its ancestor. Furthermore, I studied the phylogeny and biology of the genus Termitomyces in more detail to understand what made the genus so diverse and successful.

In order to make inferences on the events leading to domestication of Termitomyces a well-supported phylogeny is needed. In chapter 2 I performed a phylogenomic analysis of 25 species of Termitomyces and 21 related non-domesticated species. To reconstruct the biology of the ancestor of Termitomyces I also studied the biology of the nondomesticated species. The phylogenomic analysis recovered the insect-faecal associated genus Arthromyces as the sister group of Termitomyces. I found that Arthromyces shares a suite of traits with Termitomyces, indicating that their common ancestor also possessed these traits. This suite of traits consists of the following: a rooting stipe 
(pseudorhiza), an insect-faecal association, the production of asexual spores (conidia), the absence of clamp connections and an enzymatic profile with a reduced potential to degrade plant-cell wall components. I hypothesize that this set of traits predisposed the ancestor of Termitomyces towards domestication. I found that these traits were not only present in Arthromyces and Termitomyces, but also to various degrees in other non-domesticated species in the termitomycetoid clade.

One of traits found in many species of the termitomycetoid clade is the production of asexual spores, also known as conidia. I hypothesized that these conidia may play a role in local dispersal of the fungus and potentially in faster substrate colonisation. In chapter $\mathbf{3}$ I conducted a population study on the insect-faecal associated fungus Blastosporella zonata, which produces conidia on the mushroom. I sequenced two highly variable genetic markers of 21 collections of $B$. zonata mushrooms at three collection sites in cloud forests near Murillo, Colombia. I found signatures of clonality within a collection site but usually not between sites, which indicates that conidia are mainly used for local dispersal. There was genetic diversity as well, particularly between collection sites, indicating an important role for sexual spores (basidiospores) and resulting in unique genotypes of most collections. This was in agreement with an experiment in which I succeeded in germinating basidiospores in the laboratory. Unexpectedly. by reconstructing fungal haplotypes and subsequent phylogenetic analysis on these, I found evidence for the existence of two cryptic biological species in $B$. zonata.

In the studies I conducted in chapter 2 I used five unidentified species of Tephrocybe. As the morphological descriptions as well as the DNA sequences of these species did not match any species already known, I chose to describe those species in chapter 4 . The taxonomy for these five new species was challenging as morphological traits and biogeography did not align with the molecular phylogeny. Therefore, I discussed several alternative options to keep genera monophyletic. This ultimately led to the erection of four new genera: Australocybe, Nigrocarnea, Phaeotephrocybe and Praearthromyces.

The genus Termitomyces contains about 40 described species and harbours a lot of morphological diversity, examples of this are $T$. titanicus, the largest mushroom in the world with a cap up to one metre in diameter and the small T. microcarpus of which the cap rarely 
exceeds two centimetre in diameter. There are also ecological differences within the genus, while the majority of species initiate fruiting underground on the fungus comb and remain attached with a rooting stipe, $T$. microcarpus fruits aboveground on comb material expelled from the nest by the termites. Probably many species of Termitomyces remain undescribed as there is a strong bias towards the description of species that regularly produce mushrooms. In chapter $\mathbf{5}$ I assembled a large dataset of over 1500 DNA sequences of Termitomyces and used species delimitation software to sort sequences into hypothetical species based on the barcoding gap within the dataset. The barcoding gap is the difference between inter- and intraspecific genetic distances within a group of organisms. Using this approach, I recovered 87 phylogenetic species and for these species I collected specimen metadata such as continent of origin and termite host genus. A phylogenetic reconstruction of the 87 species revealed five main symbiont groups which corresponded with the five largest fungus-growing termite genera. It also revealed a single origin of the epigeous fruiting habit of $T$. microcarpus and at least seven intercontinental migrations. I identified several factors which could be involved in speciation: fruiting mode (underground or aboveground), geographic separation, symbiont transmission mode and suppression of mushroom formation by the host termite. Finally, I demonstrated that mushroom morphology does not correlate with phylogeny and therefore, without additional support of molecular data, is not useful for taxonomy in this genus.

Uniparental vertical transmission (transmission from parent to offspring) of symbionts is an important factor in aligning reproductive interests of host and symbiont and thus stabilizing the symbiosis. In fungus-growing termites, horizontal transmission (symbiont obtained from the environment) is the predominant transmission mode, but uniparental vertical transmission has evolved via the female reproductives of the genus Microtermes and via the male reproductives of the species $M$. bellicosus. In chapter $\mathbf{6}$ I studied populations of Microtermes and their symbionts in South Africa. Although in theory uniparental vertical transmission leads to decreased genetic symbiont diversity and stronger host-symbiont relationships, I found high genetic diversity in symbiont populations and low host-specificity, which indicates that frequent horizontal symbiont exchange occurs as well as sexual reproduction, despite the absence of mushrooms of these species. I therefore argue that Microtermes species regularly acquire symbionts 
associated with species of other genera that do not suppress mushroom formation, such as species of the genus Ancistrotermes.

New species of mushroom-forming fungi are often described based on morphological features of the mushroom. In the genus Termitomyces this has caused a heavy bias towards the description of species, which regularly produce mushrooms, leaving species that rarely or infrequently fruit undescribed. In chapter $\mathbf{7}$ I describe, for the first time in this genus, a species of Termitomyces with no reported naturally produced mushrooms, based on DNA sequence evidence, biological data and asexual features.

Fungiculture has evolved independently several times in insects. How fungiculture can evolve and which conditions are required remain poorly understood. In chapter 8, I discuss how investigating and understanding the biology of species closely related to fungal symbionts can assist in reconstructing the biology of the ancestor and thereby determine conditions that facilitated fungal domestication by an insect. Although my research focused on a specific group of fungusgrowing insects, the fungus-growing termites, I discuss how my approach can be applied to different unrelated groups of fungusgrowing insects as well. Finally I discuss how my work not only offers significant contributions to the knowledge on the origin and subsequent evolution of fungiculture in termites, but also contributed to general fundamental knowledge of mutualistic symbioses in general. 


\section{Acknowledgements}

The longest ultramarathon I have ever participated in has now come to an end. Finishing a race is never a complete solo-effort as it requires the assistance of a good support team and various aid stations along the way. Here I would like to thank my support team and all the other people at the various aid stations that have made this finish possible.

First of all I would like to thank my supervisor: Duur. One of the first things you asked me when I started my PhD was whether I was interested in working on a bunch of peculiar fungi with a possible insectassociation in the cloud forests of central and south America. The mystery surrounding these fungi immediately grabbed my intention and I consider myself lucky I could work on this topic. The fieldwork trips to Colombia, Puerto Rico and South Africa were the true highlights of my PhD. I really enjoyed the freedom you gave me in my work by pursuing my own ideas and directions within this topic of research. I learnt a lot from your very creative approach to science as well as your excellent writing skills.

Bas, it has been great having you as a boss and promotor. I have learned a lot from your critical attitude and the discussions we had about science.

Mathijs, like the fungus-growing termites, our work is a prime example of a mutualistic symbiosis. I really enjoyed working and discussing with you and I think we made a great team. Our excellent shared thesis chapter, which is now published in Current Biology, is a perfect demonstration of this. I also really enjoyed our trip to the KEW botanical gardens and herbarium in London.

The people at the Laboratory of Genetics always made me feel welcome and at home. I would like to thank some people in particular, starting with Wytske. Besides all your help with forms and bureaucracy I valued your involvement and the social cohesion you provide to the group. I want to thank Bertha and Marijke for their assistance in starting up my experimental work in the lab. Alex, Anna, Eveline, Fons, Jianhua, Kim, Anneloes, Ramon, René, Roel and Tom, thanks for interesting conversations and keeping me motivated. Cris, I was happy to be your paranymph when you defended your thesis. I also want to thank you for your friendship and getting me into (ultra)running. 
I would also like to thank the previous and current members of group Duur: Eric, Sabine, Margo and Anouk. Thank you for great discussions, interesting talks and all your constructive feedback on my work.

Alex, I am really glad you joined the fieldtrip to Colombia and Puerto Rico. I had a really great time and it was fun sharing this adventure with you. Ben, I really enjoyed working with you. I learned a lot from you about all kinds of computer programs, but also about science in general and bike racing. I also enjoyed all the long bike rides that we did, even after half a day of cycling we never ran out of stuff to talk about. I am glad that you accepted to be paranymph at my defence.

I want to thank my students Natascha and Jeroen for choosing me as their supervisor and for their contributions to my work.

My $\mathrm{PhD}$ research started with a simple email from Tim Baroni who discovered some interesting fungi, probably closely related to Termitomyces, which were growing on insect poop. It is crazy to imagine that this single email led to two separate field trips in south America, more than five years of research and an entire PhD thesis. Tim, it was a pleasure to spend time with you in the jungle, sip rum together and to learn all kinds of new things about fungi and their taxonomy.

National and international collaborations have been crucial during my research and I therefore want to thank Esperanza and Martha for setting up and assisting with the field and lab work in Colombia and Cathie, Thomas, Ole and Dirk for their help in collecting samples and writing up the taxonomy. Wilhelm, thanks for being a great host when we came to Pretoria for field work. I also want to thank you for your help in the herbarium and teaching me about taxonomy. Thom, thank you for all your advice and constructive criticism during the development of my PhD project. I also want to thank you for your help with fungal sampling and your super detailed answers to all my questions related to fungal taxonomy. Peter Biedermann, thanks for fruitful collaborations and for your interest in my work.

The direct and indirect mental support from my group of close friends has been vital during my entire PhD but became even more important in the final stages. First of all, I want to start with thanking the 'Herpemerpederpeflerp' biologist crew: Jelle, Jery, Kirstin, Lisanne, Marcus, Melanie, Sjors and Tim. Thanks for big lunches and dinners, lots of coffee, lengthy discussions and rants, barbecues, walks, swims, holiday trips, new year celebrations and motivating me to keep going.

I want to thank the distance skateboarding and ultraskate community, in particular: Anton, Daniëlle, Joey, Jonathan, Pat, Rick 
and Stan. Thank you for all the amazing skate vacations including trips to Berlin, the French alps, the Pyrenees, Italy, Tenerife and Miami. Luutse, thanks for your friendship and the exciting times building boards together. Kimmy, thanks for your hospitality and sharing tasty craft beers. Bas, thanks for great skate sessions, your support at the Dutch Ultraskates and for a long friendship. Andy, thanks for your friendship, inspiring me as an endurance athlete and for being a total badass. I want to thank the Track \& Trailbusters running group for enjoyable training sessions and improving my running skills. I want to thank Harmen, Ivo and Joep in particular, for all the Sunday excursions and Tycho for his training advice and coaching.

Thanks to the Roadburn festival buddies: Dirk, Jeroen and Tim for enjoying good music together, drinking tasty beers and consuming many Lihanboutjes. Erik-Jan, thanks for many years of friendship and your support at the Dutch Ultraskate. Although we don't see each as much as we used to, I enjoy sharing stories every time we meet. Also thanks to Roemlah and Jan for your hospitality. Your place always feels like a second home to me.

Mom, Nicoline, thanks for all your love and support. I also want to thank you for a listening ear during difficult times and for sharing happiness and joy during all the good times.

Dad, Peter, thanks for your listening ear, dry humour and for our bike rides together. As an evolutionary biologist and a pastor, I think we did a great job in looking for the things that unite us rather than divide us.

Dr. doctor and brother Ivo, I am glad we can both call ourselves doctors now! Thanks for the many great things we shared: festivals, concerts, whisky and beer tastings, ultraskates and bike rides. I am happy to have you at my side as a paranymph at the defence.

John, Joke and Stijn, thanks for your hospitality, fun holiday trips, mountainbike rides and your assistance at the Dutch Ultraskate.

Lonneke, thank you for all your love and support, and for the many wonderful years we spent together. 



\section{PE\&RC Training and Education}

Statement

With the training and education activities listed below the $\mathrm{PhD}$ candidate has complied with the requirements set by the C.T. de Wit Graduate School for Production Ecology and Resource Conservation (PE\&RC) which comprises of a minimum

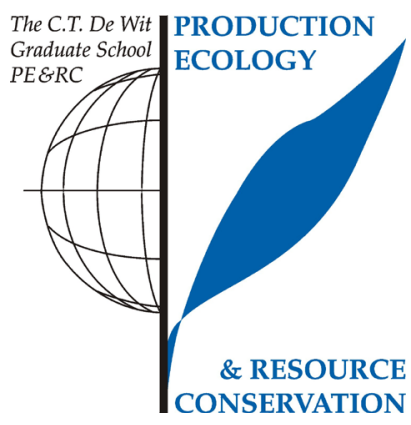
total of 32 ECTS (= 22 weeks of activities)

\section{Review of literature (4.5 ECTS)}

- Origin and evolutionary stability of the mutualism between fungusgrowing termites and their fungal symbionts

\section{Post-graduate courses (2.7 ECTS)}

- Phylogenetics: principles \& methods; BIS (2018)

- Lorentz workshop: distinguishing science and metaphysics in evolution and religion; VU (2018)

\section{Laboratory training and working visits (3 ECTS)}

- Termitomyces collecting, fieldwork and training in fungal taxonomy; FABI, Pretoria, South Africa (2015)

- Blastosporella collecting and fieldwork; University of Antioquia, Medellín, Colombia (2016)

\section{Invited review of (unpublished) journal manuscript (1 ECTS)}

- Fungal Ecology: sexual reproduction and saprotrophic dominance by the ambrosial fungus Flavodon subulatus (= Flavodon ambrosius) (2019)

\section{Competence strengthening / skills courses (2.4 ECTS)}

- Teaching and supervising thesis students, WU (2017)

- Network training, Job Participation Support WUR (2021)

- Rubicon Proposal Writing (2021)

\section{Scientific integrity/ethics in science activities (0.9 ECTS)}

- Ethics in plant and environmental sciences; WGS (2019)

- Scientific integrity; WGS (2019) 
PE\&RC Annual meetings, seminars and the PE\&RC weekend (1.2 ECTS)

- PE\&RC Day (2015-2020)

Discussion groups / local seminars or scientific meetings (4 ECTS)

- Wageningen Evolution and Ecology Seminars (WEES) (2015-2020)

- KNVM section mycology, fall meeting (2015-2020)

- Publish for impact symposium (2017)

\section{International symposia, workshops and conferences (7.6 ECTS)}

- ESEB XVI Congress; poster presentation; Groningen (2017)

- International Mycological Congress 11 (IMC); poster presentation; San Juan, Puerto Rico 92018)

- NLSEB; poster presentation; Ede (2018)

- NLSEB; poster presentation; online (2021)

\section{Societally relevant exposure (0.3 ECTS)}

- WUR Campus Safari, presentation on fungus-growing termites for kids (2018)

\section{Lecturing/supervision of practicals/tutorials (3.6 ECTS)}

- Genetic analysis tools and concepts (2015)

- Evolution and systematics (2016)

\section{$\mathrm{BSc} / \mathrm{MSc}$ thesis supervision (4 ECTS)}

- Jeroen Groeneveld MSc thesis: phylogenetic analysis of mitochondrial plasmids in Termitomyces indicates lateral transfer.

- Natascha Oosterwijk; BSc thesis: sporulation of lyophylloid agarics under different circumstances, a comparison of Arthromyces, Blastosporella, Tephrocybe and Termitomyces. 
The research in this thesis was carried out at the Laboratory of Genetics at Wageningen University \& Research, Wageningen, the Netherlands. The work was financially supported by the Netherlands Organisation for Scientific Research (VICl: NWO 86514007).

Cover photo by Leonard J.J. van de Peppel

Thesis layout by Leonard J.J. van de Peppel

Printed by DuurzameDissertatie.nl on $100 \%$ recycled paper using water-based ink.

A tree has been planted for every copy of this thesis. 
